\title{
Alexandrie (actions du Centre d'études alexandrines, 2020)
}

Aude Simony, Kathrin Machinek, Georges Soukiassian, Valérie Pichot, Ismaël Awad, Mohamed Abdelaziz, Mohamed Elsayed, Isabelle Hairy et Philippe Soubias

\section{OpenEdition}

Journals

Édition électronique

URL : https://journals.openedition.org/baefe/2885

DOI : $10.4000 /$ baefe.2885

ISSN : 2732-687X

Éditeur

ResEFE

Référence électronique

Marie-Dominique Nenna, Aude Simony, Kathrin Machinek, Georges Soukiassian, Valérie Pichot, Ismaël Awad, Mohamed Abdelaziz, Mohamed Elsayed, Isabelle Hairy et Philippe Soubias, « Alexandrie (actions du Centre d'études alexandrines, 2020) » [notice archéologique], Bulletin archéologique des Écoles françaises à l'étranger [En ligne], Égypte, mis en ligne le 30 mai 2021, consulté le 27 juillet 2021. URL : http://journals.openedition.org/baefe/2885 ; DOI : https://doi.org/10.4000/baefe.2885

Ce document a été généré automatiquement le 27 juillet 2021

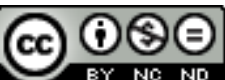

Le Bulletin archéologique des Écoles françaises à l'étranger est mise à disposition selon les termes de la Licence Creative Commons Attribution - Pas d'Utilisation Commerciale - Pas de Modification 4.0 International. 


\title{
Alexandrie (actions du Centre d'études alexandrines, 2020)
}

\author{
Aude Simony, Kathrin Machinek, Georges Soukiassian, Valérie Pichot, \\ Ismaël Awad, Mohamed Abdelaziz, Mohamed Elsayed, Isabelle Hairy et \\ Philippe Soubias
}

\section{NOTE DE L'AUTEUR}

Etablissements porteurs du projet : CEAlex, USR $3134 \mathrm{Cnrs} / \mathrm{IFAO}$

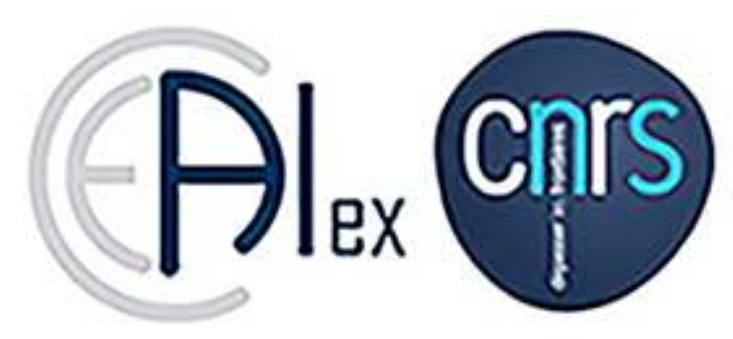

En 2020, malgré la pandémie de Covid-19, le Centre d'études alexandrines (CEAlex, USR 3134, CNRS/Ifao) a été à même de poursuivre les fouilles sur le site de Kôm Bahig et sur la villa agricole du site d'Akadémia, de même que ses prospections en Maréotide dans la région au sud du lac Mariout, et a mené des campagnes de fouilles sous-marines sur le site du Phare et sur l'épave QB2 (fig. 1). Il a poursuivi ses activités de recherche et de préparation de publications consacrées aux fouilles de sauvetage effectuées à Alexandrie et au mobilier qui en est issu ${ }^{1}$ et a continué de participer au programme VICI Innovating objects en collaboration avec l'université de Leyde et au programme Water Traces (Amidex). Le CEAlex a également développé ses actions portant sur Alexandrie moderne en collectant des archives de familles et d'institutions alexandrines et en poursuivant le programme Presse francophone d'Égypte. 
Fig. 1. Carte de localisation des activités de terrain du CEAlex en 2020 (V. Pichot).

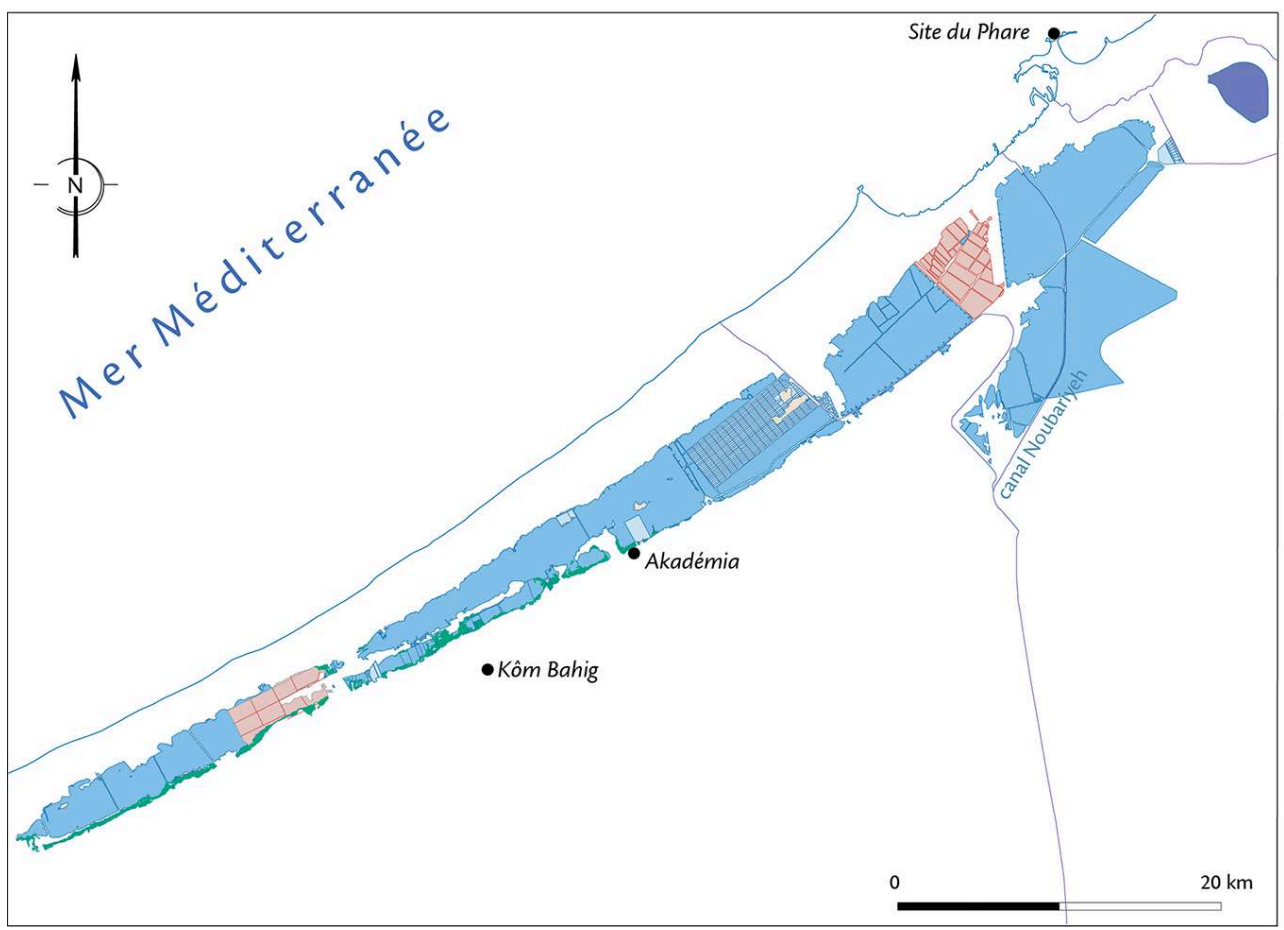

(c) Archives CEAlex. CEAlex_BAH_2020_NDMCN_001

2 La valorisation de ses fonds et de ses recherches a été effectuée à travers la participation au programme "Portail des Bibliothèques d'Orient » piloté par la BNF, grâce au programme EGYNUM dans le cadre du Collex Persée. Les onzièmes journées du patrimoine alexandrin que le CEAlex coordonne se sont tenues en virtuel. Les livrets issus de deux expositions créées par le CEAlex qui devaient accompagner l'anniversaire des 30 ans du CEAlex ont également paru ${ }^{2}$. Le site web du CEAlex a été entièrement refondu (www.cealex.org).

Trois thèses portant sur le mobilier issu des fouilles du CEAlex ou s'insérant dans les programmes de recherches de l'équipe ont été soutenues en 2020 : Nicolas Morand, Approche archéozoologique de la ville d'Alexandrie de sa fondation à l'époque moderne (IV siècle av. J.-C. - XVIII siècle apr.J.-C. (MNHN, direction Sébastien Lepetz, Benoît Clavel, MarieDominique Nenna); Ismaël Awad, La marge occidentale du delta du Nil. Système d'Information Géographique sur les traces de l'occupation antique de la Maréotide (université Lumière Lyon 2, direction Jean-Yves Empereur ; Bassem Ibrahim, Les sites archéologiques autour d'Alexandrie: Canopus et Maréotis. Une étude d'aménagement du site à la lumière de leurs caractéristiques archéologiques (direction Jean-Yves Empereur et Mona Haggag, université d'Alexandrie).

4 Romain Séguier a été recruté comme ingénieur de recherche archéologue et a pris son poste au $1^{\text {er }}$ décembre 2020.

\section{Kôm Bahig}

Aude Simony, Kathrin Machinek et Georges Soukiassian Responsable d'opération: Aude Simony (archéologue-céramologue, CNRS, CEAlex) assistée par Shady Morsi (contremaître, CEAlex) avec une vingtaine d'ouvriers. 
Participants : Kathrin Machinek (archéologue-architecte, CNRS, CEAlex), Georges Soukiassian (archéologue, CNRS, CEAlex), Cécile Shaalan (ingénieur topographecartographe, CNRS, CEAlex), Ismaël Awad (ingénieur topographe-cartographe, CEAlex), Ragab Wardani (assistant topographe, CEAlex), Philippe Soubias (photographe, CNRS, CEAlex), Mahmoud Fathy (relevés de terrain, CEAlex), Hisham Mohamed Mahmoud Aboud (gestion du mobilier, CEAlex), Ahmed Elsayed Mohamed Hassan El Nagar (dessin du mobilier, CEAlex), Walid Abdel Bahry (tri et comptage de la céramique), sherine El Sayed et Aly Ahmed (supervision de l'opération de levage et de déplacement des blocs calcaires).

Autorité nationale présente : Le ministère du Tourisme et des Antiquités de l'Égypte (MoTA) était représenté par Ahmed Mahmoud Mohamed Shalaby (16 février 2020-16 mars 2020) et Amr Ibrahim El Sayed Mohamed Mansour (16 mars 2020 - 23 mars 2020).

Les travaux menés sur le site de Kôm Bahig bénéficient du soutien du ministère de l'Europe et des Affaires étrangères (quadriennal 2020-2023).

5 Kôm Bahig se situe au sud de la ride pléistocène (III) de Gebel Mariout, à environ $1 \mathrm{~km}$ du lac (WGS84 latitude $30.933587^{\circ} \mathrm{N}$; longitude $29.587410^{\circ} \mathrm{E}$; carte Egyptian Survey au 1:50 000 : NH35-L6c : Burj el-Arab) recensé dans l'Archaeological GIS Project (Cultnat) sous le numéro 110322 (voir fig. 1). Il s'étend sur environ $600 \mathrm{~m}$ nord/sud $\times 350 \mathrm{~m}$ est/ ouest (fig. 2). Une vallée longue et étroite ( $200 \mathrm{~m} \mathrm{nord} / \mathrm{sud} \times 30 \mathrm{~m}$ est/ouest), sans doute l'emplacement d'une voie, est bordée par deux kôms d'environ $10 \mathrm{~m}$ de hauteur, formés par l'accumulation des couches archéologiques. Au nord se trouve une zone rectangulaire basse $(150 \mathrm{~m}$ nord/sud $\times 110 \mathrm{~m} \mathrm{E} / \mathrm{W})$, peut-être l'emplacement d'un temple. Le CEAlex réalise des fouilles sur ce site depuis $2016^{3}$.

Fig. 2. Kôm Bahig. Image satellite du site.

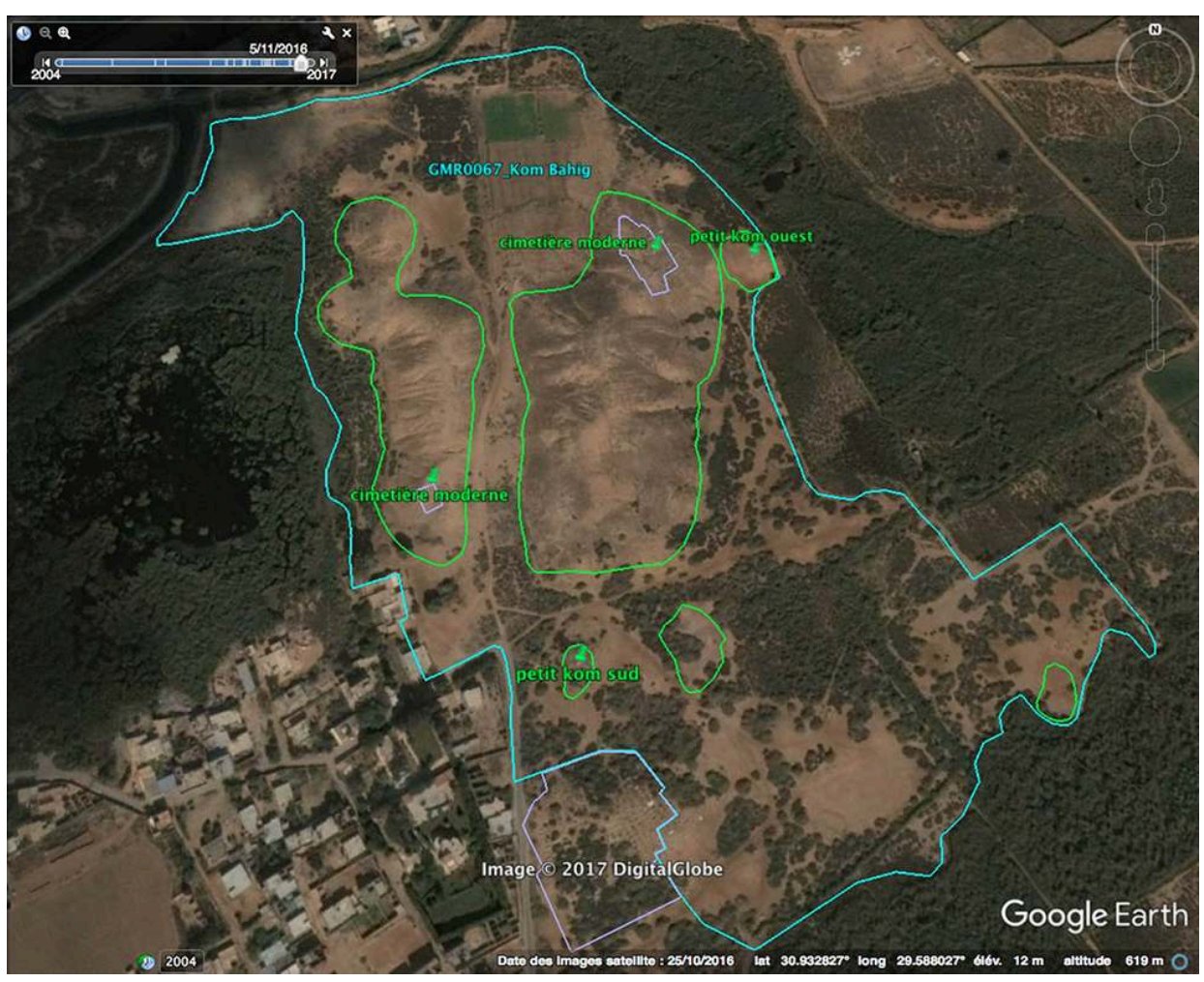

(C) GoogleEarth. CEAlex_BAH_2020_NDMCN_002 
6 La cinquième campagne de fouilles menée sur le site de Kôm Bahig s'est déroulée du 16 février au 23 mars 2020. Cette campagne devait initialement s'achever à la miavril 2020. En raison de la crise sanitaire liée à l'épidémie de la Covid-19, les travaux de fouille ont été suspendus le 23 mars 2020. Ces travaux se sont concentrés sur le sondage 20000 , localisé dans la partie nord de la vallée centrale traversant le site du nord au sud (fig. 3). La campagne a débuté par une opération de levage des gros blocs de pierre calcaire qui avaient, pour la plupart, été dégagés dès 2016 et ce, dans l'optique de trouver le raccord entre le dallage d'orientation N-S situé dans la partie sud du sondage (20014) et dégagé en 2016 et le dallage d'orientation E-W situé au nord (20029) et dégagé en 2019 (fig. 4).

Fig. 3. Kôm Bahig. Plan général du site de Kôm Bahig et localisation du sondage 20000 (service de topographie du CEAlex).

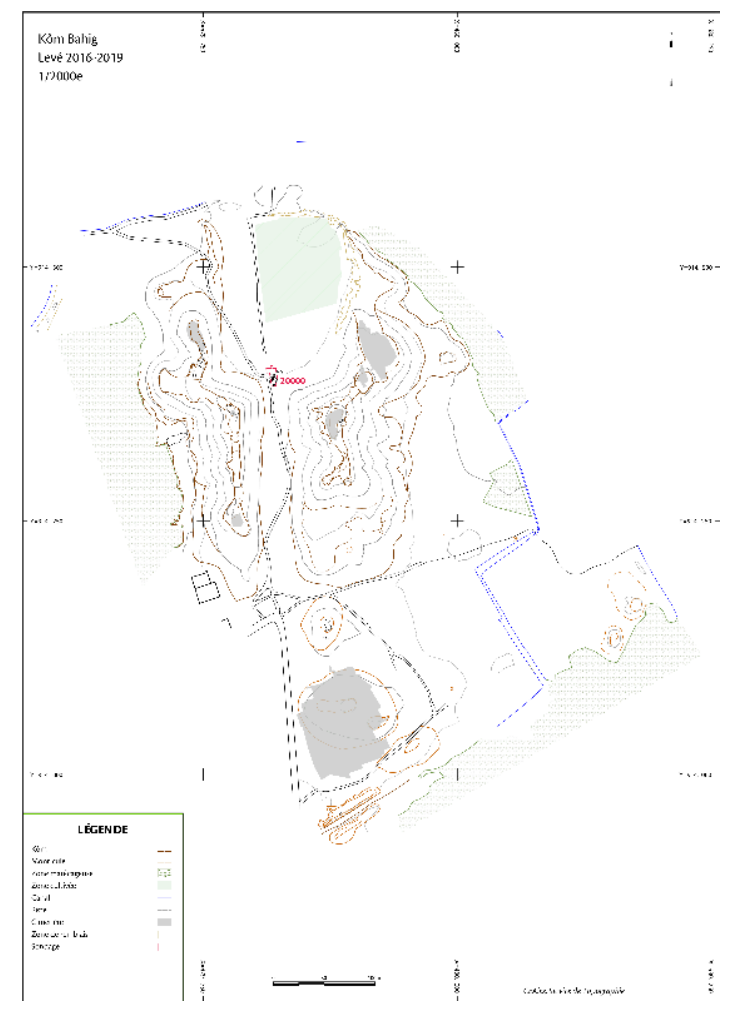

(C) Archives CEAlex. CEAlex_BAH_2020_NDMCN_003 
Fig. 4. Kôm Bahig. Plan du sondage 20000 avant l'extraction des gros blocs calcaires (fouilles 2016-2019) (K. Machinek, A. Simony, G. Soukiassian).

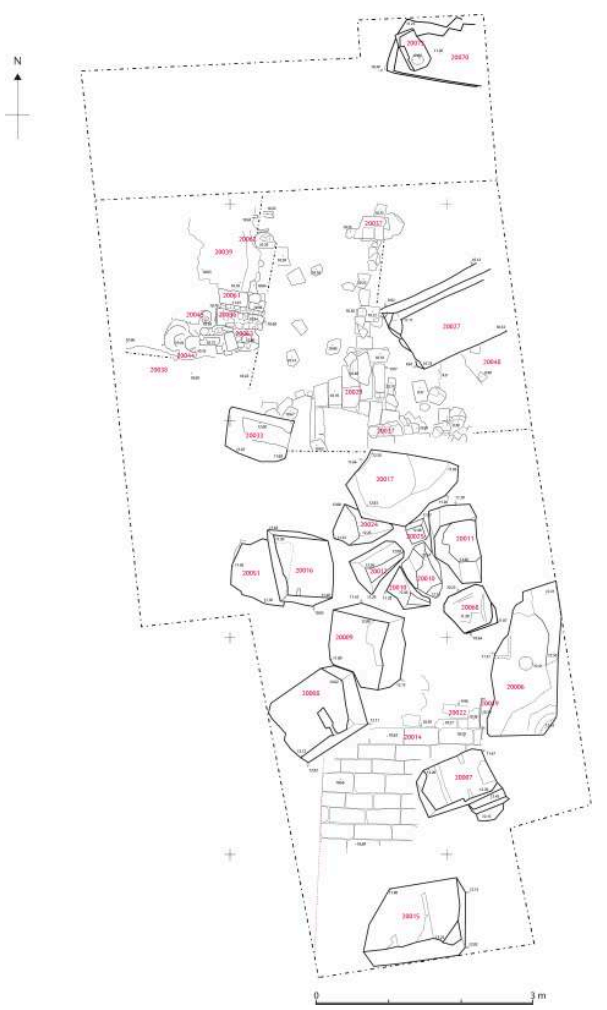

\subsection{Levage et étude architecturale des blocs de pierre du sondage 20000}

7 Nous avons procédé au levage des gros blocs en calcaire du secteur 20000 afin de pouvoir fouiller les couches scellées jusqu'alors par ces blocs. Pour mener à bien cette tâche, nous avons fait appel à une grue mobile et une semi-remorque. Nos spécialistes ont attaché les sangles correspondant au poids des blocs pour un levage en sécurité (fig. 5). Ainsi, nous avons pu lever 13 blocs, dont 11 blocs monumentaux et 2 blocs de taille moyenne. Les blocs monumentaux pèsent entre 2,8 tonnes et 11,14 tonnes (voir tableau ci-dessous). Ils ont été disposés à l'entrée du site, le long de la piste des véhicules qui traverse le site du sud au nord, sur des madriers de bois (fig. 6). 
Fig. 5. Kôm Bahig. Levage des gros blocs de calcaire du sondage 20000 le 20 février 2020 (A. Simony).

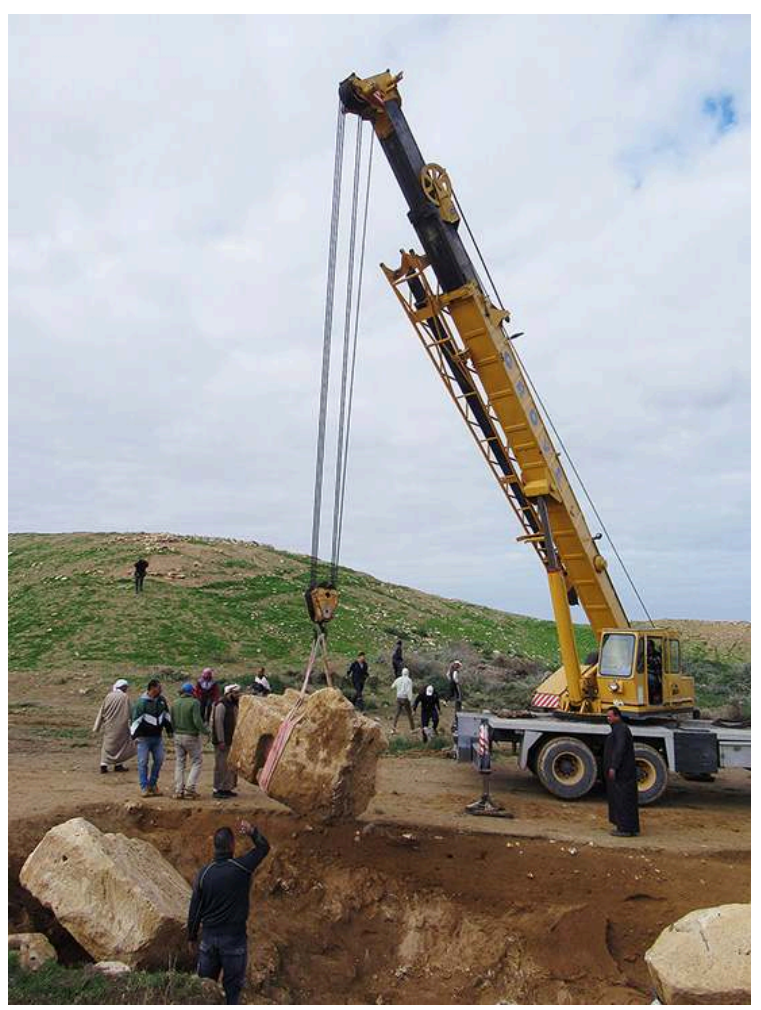

(c) Archives CEAlex. CEAlex_BAH_2020_NDMPF_001

Fig. 6. Kôm Bahig. Emplacement actuel des blocs du sondage 20000, à l'entrée du site (K. Machinek).

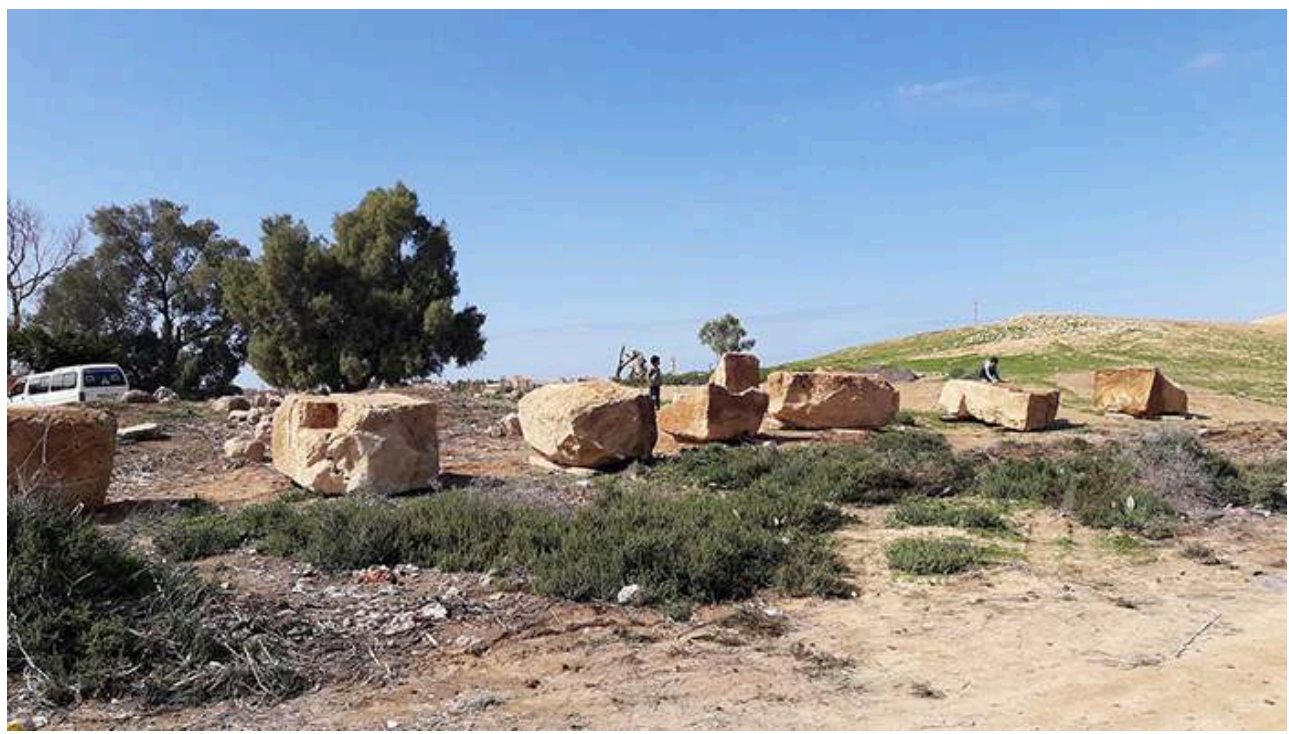

(c) Archives CEAlex. CEAlex_BAH_2020_NDMPF_002

\begin{tabular}{|l|l|l|l|l|}
\hline Bloc & Longueur $\mathbf{( c m )}$ & Largeur $\mathbf{( c m )}$ & Hauteur $\mathbf{( c m )}$ & poids $(\mathbf{t})$ \\
\hline 20007 & 190 & 110 & 108 & 6,09 \\
\hline 20008 & 200 & 160 & 110 & 9,50 \\
\hline
\end{tabular}




\begin{tabular}{|c|c|c|c|c|}
\hline 20009 & 175 & 157 & 108 & 8,01 \\
\hline 20010-1 & 100 & 76 & 93 & 1,91 \\
\hline 20010-2 & 98 & 104 & 72 & 1,98 \\
\hline 20011 & 153 & 110 & 100 & 4,54 \\
\hline 20012 & 125 & 92 & 110 & 3,42 \\
\hline 20015 & 246 & 167 & 80 & 8,87 \\
\hline 20016 & 152 & 150 & 95 & 5,85 \\
\hline 20017 & 250 & 150 & 110 & 11,14 \\
\hline 20024 & 120 & 96 & 90 & 2,80 \\
\hline 20025 & 70 & 40 & 42 & 0,32 \\
\hline 20049 & 74 & 38 & 31 & 0,24 \\
\hline
\end{tabular}

8 Au cours de la campagne de 2020, d'autres blocs appartenant à l'ensemble de gros blocs en calcaire ont été mis au jour (20051, 20068 et 20070). Nous avons continué la documentation et les relevés architecturaux des faces des blocs monumentaux à échelle 1:20. Six blocs monumentaux demeurent dans le sondage 20000 pour l'instant, puisqu'ils ne gênent pas les travaux de fouilles.

\begin{tabular}{|l|l|l|l|l|}
\hline Bloc & Longueur $\mathbf{( c m )}$ & Largeur $\mathbf{( c m )}$ & Hauteur $\mathbf{( c m )}$ & Poids (t) \\
\hline 20006 & 350 & 160 & 100 & 15,12 \\
\hline 20027 & 280 & 150 & 130 & 14,74 \\
\hline 20033 & 146 & 102 & 122 & 4,91 \\
\hline 20051 & 150 & 135 & 53 & 2,90 \\
\hline 20068 & 118 & 95 & 100 & 3,03 \\
\hline 20070 & 220 & 145 & 110 & 9,47 \\
\hline
\end{tabular}

9 D'autres blocs de taille moyenne ont été retirés de la fouille et entreposés à côté du sondage 20000.

\begin{tabular}{|l|l|l|l|l|}
\hline Bloc & Longueur $\mathbf{( c m )}$ & Largeur $(\mathbf{c m})$ & Hauteur $(\mathbf{c m})$ & Poids (t) \\
\hline 20050 & 80 & 40 & 43 & 0,37 \\
\hline 20058 & 60 & 36 & 30 & 0,17 \\
\hline 20059 & 77 & 35 & 33 & 0,24 \\
\hline 20073 & 89 & 51 & 46 & 0,56 \\
\hline
\end{tabular}




\subsection{Fouille du sondage 20000}

Une fois l'opération de levage terminée, la fouille des niveaux situés directement sous les gros blocs calcaires a pu débuter (fig. 7-8). Comme le suggérait déjà la présence des gros blocs et du dallage 20014, il se confirme que le sondage 20000 se trouve aux abords d'un sanctuaire.

Fig. 7. Kôm Bahig. Sondage 20000 après extraction des blocs calcaires (K. Machinek, G. Soukiassian).

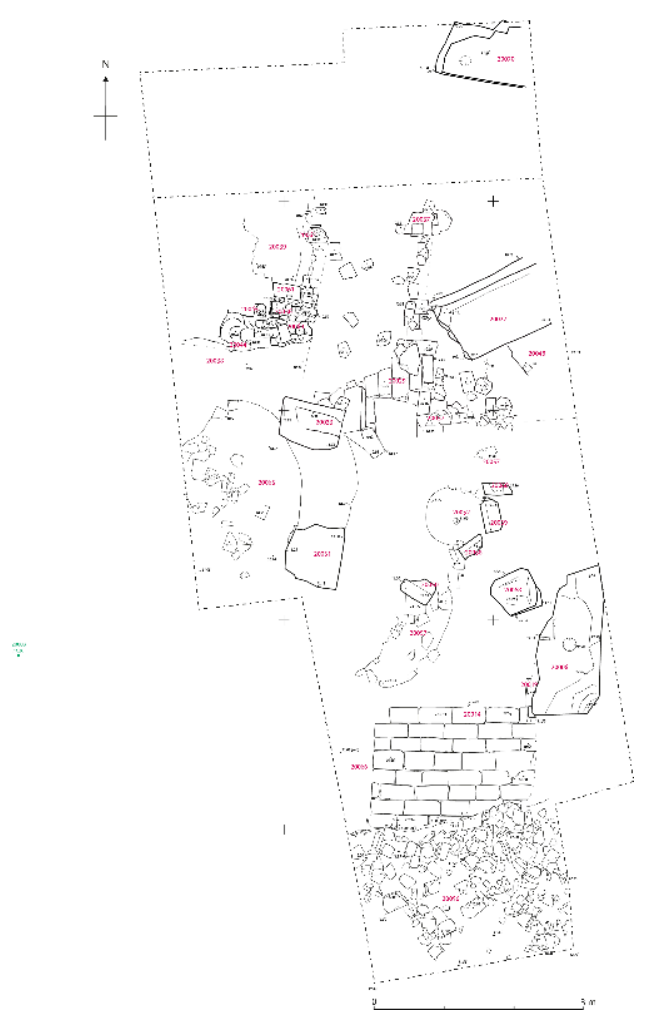

(c) Archives CEAlex. CEAlex_BAH_2020_NDMCN_005 
Fig. 8. Kôm Bahig. Sondage 20000 après extraction des blocs calcaires. (M.-D. Nenna).

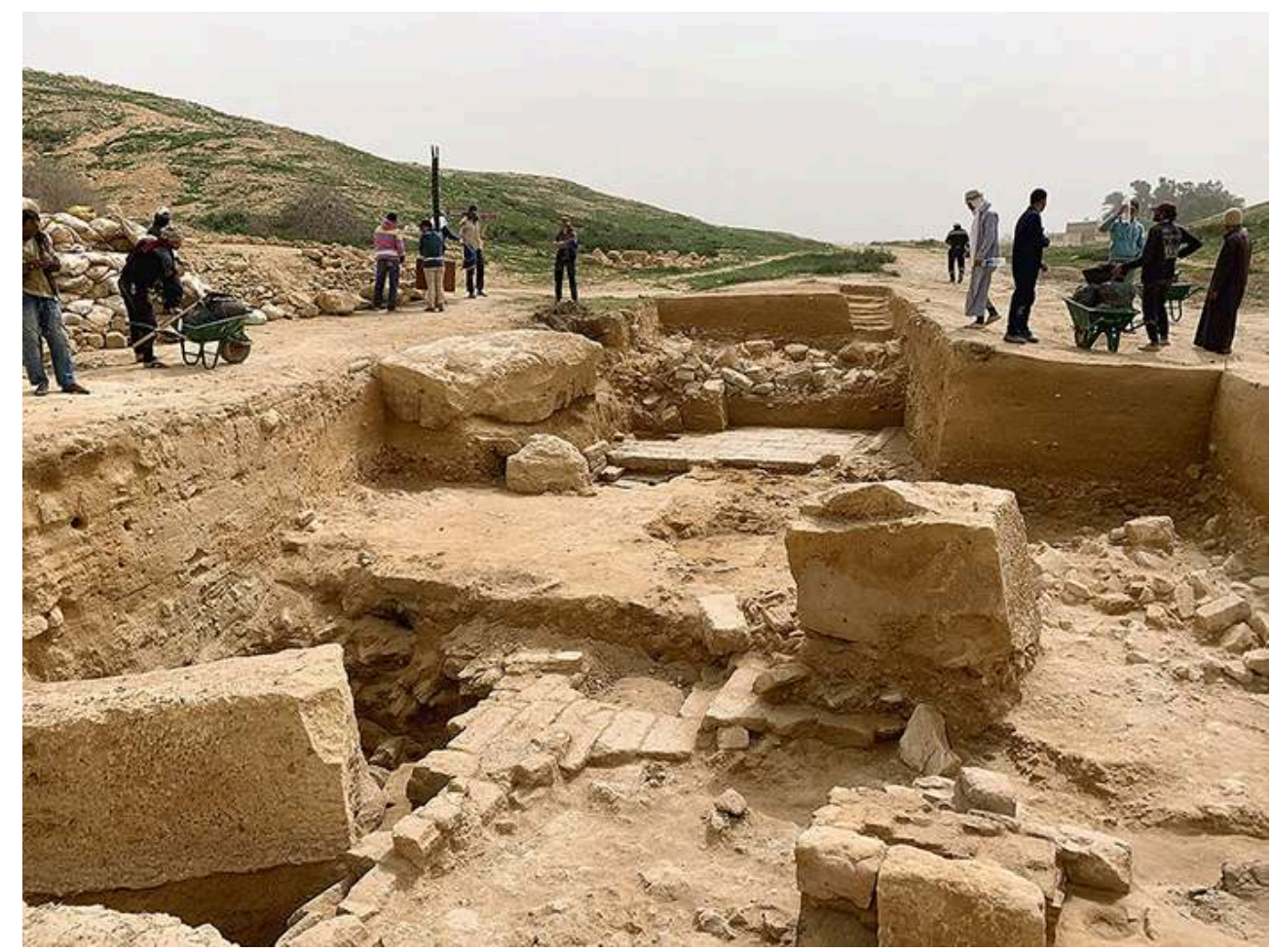

(c) Archives CEAlex. CEAlex_BAH_2020_NDMPF_003

\subsubsection{Sondage 20000 sud}

11 Dans la partie sud du sondage, les blocs 20007 et 20015 reposaient sur une succession de niveaux de débris de démolition composés d'une accumulation de blocs calcaires (US 20056, 20066 et 20076). La couche 20056 (fig. 9) contenait près de 100 petits blocs calcaires, dont une quarantaine de dalles. Cependant, aucun élément architectural particulier, sculpté ou décoré, n'a été reconnu dans cette US. Nous avons numéroté et retiré les blocs, avant de les entreposer à l'extérieur du sondage 20000 pour pouvoir les enregistrer et documenter.

12 Ce lot de pierres à bâtir d'un calcaire tendre et crayeux montre de fortes traces de détérioration et de cassures. Parmi les moellons les mieux conservés, nous avons pu évaluer des dimensions d'environ $55 \mathrm{~cm}$ pour les longueurs, $35 \mathrm{~cm}$ pour les largeurs et $25 \mathrm{~cm}$ pour les hauteurs. Les dalles ont des épaisseurs de $9 \mathrm{~cm}$ en moyenne, comme celles du dallage du dromos. Certains blocs gardent encore des pans de mortier de liant au niveau de leurs joints.

$13 \mathrm{Au}$ niveau de la limite ouest du dallage 20014, les vestiges d'une bordure calcaire (20085) et d'un dispositif d'encastrement (20084) ont été dégagés. Ces deux installations sont coupées à l'ouest par la fosse 20074 (fig. 10). 
Fig. 9. Kôm Bahig. Couche de démolition 20056, vue depuis le sud (G. Soukiassian).

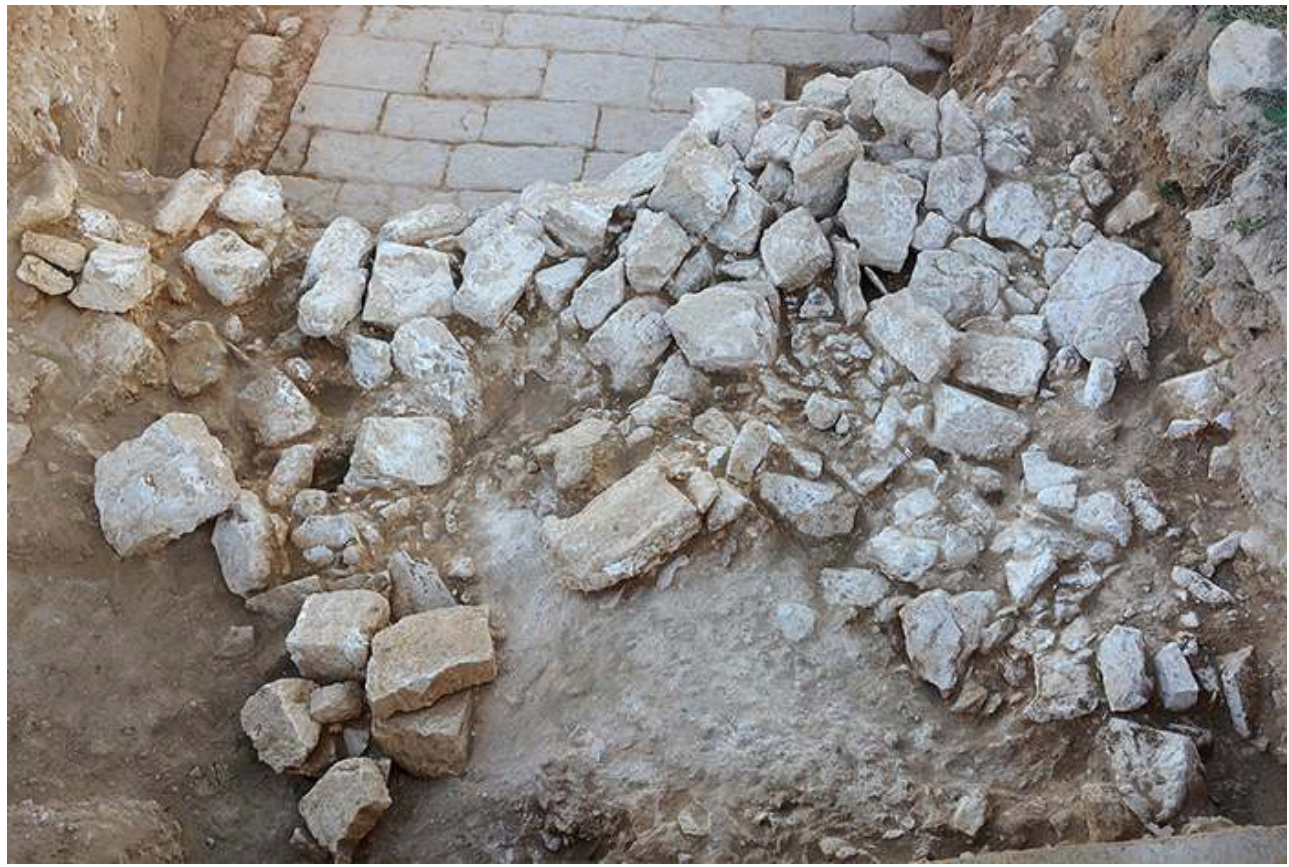

(c) Archives CEAlex. CEAlex_BAH_2020_NDMPF_004

Fig. 10. Kôm Bahig. Le dispositif d'encastrement 20084 et la bordure calcaire 20085 à l'ouest du dallage 20014, vue depuis le sud-est (G. Soukiassian).

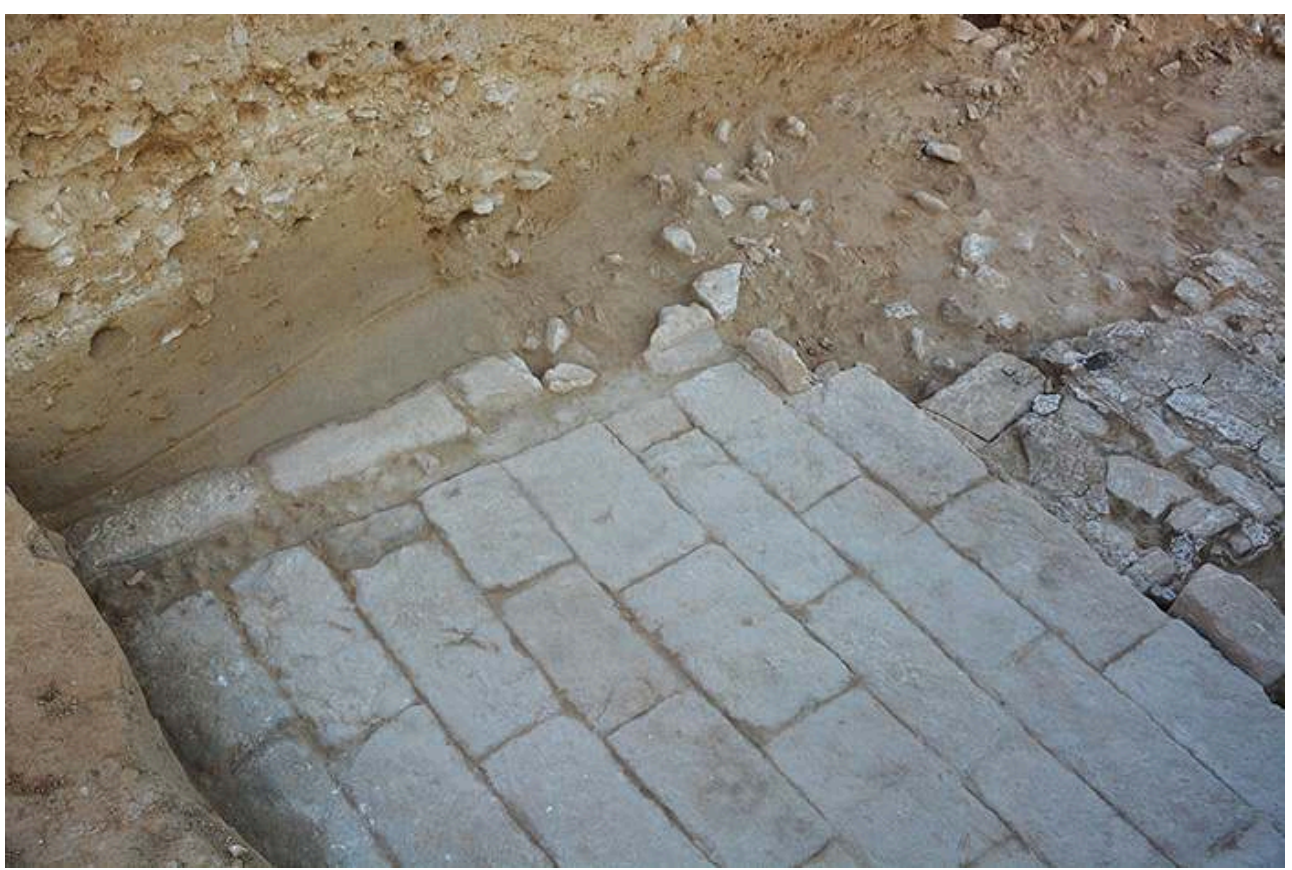

(c) Archives CEAlex. CEAlex_BAH_2020_NDMPF_005

14 Directement au nord du dallage 20014, une couche de débris de démolition de même nature que 20056 (US 20057 - fig. 11) ainsi qu'un nouveau bloc calcaire (20051) sont apparus directement sous les blocs 20008, 20009, 20010, 20011, 20012, 20016, 20017, 20024 et 20025. Un sol d'occupation délimité par de petits blocs de calcaire disposés en arc de cercle (20052 - fig. 12) et correspondant vraisemblablement à l'emplacement 
d'un aménagement temporaire à piquet ou petit poteau central (de type tente ou abri) a également été mis au jour. Cet ensemble reposait sur le niveau de démolition 20067 composé de débris calcaires, de fragments d'enduit et de mortier mêlés à de poches terreuses. Il contenait de nombreux fragments de calcaire inscrits ou décorés, des fragments de porphyre et de granit (voir § 3 ) et pourrait ainsi correspondre à une phase de débitage de petits édifices situés à l'origine de part et d'autre du dromos.

Fig. 11. Kôm Bahig. Couche de démolition 20057, vue depuis le sud-ouest (G. Soukiassian).

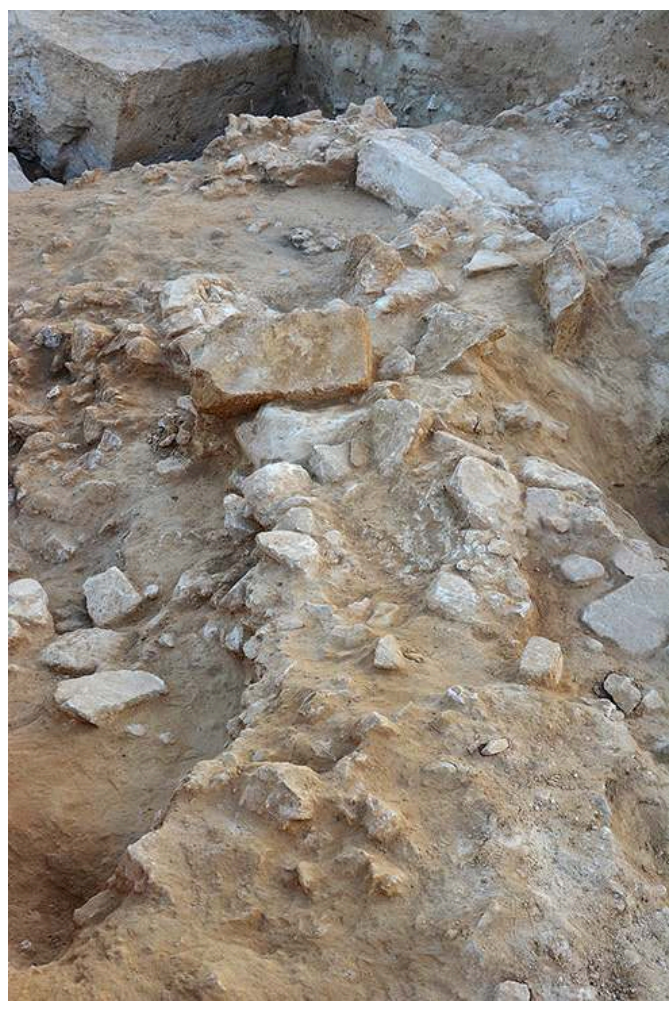

(C) Archives CEAlex. CEAlex_BAH_2020_NDMPF_006 
Fig. 12. Kôm Bahig. Emplacement de l'installation temporaire 20052, vue depuis le sud (G. Soukiassian).

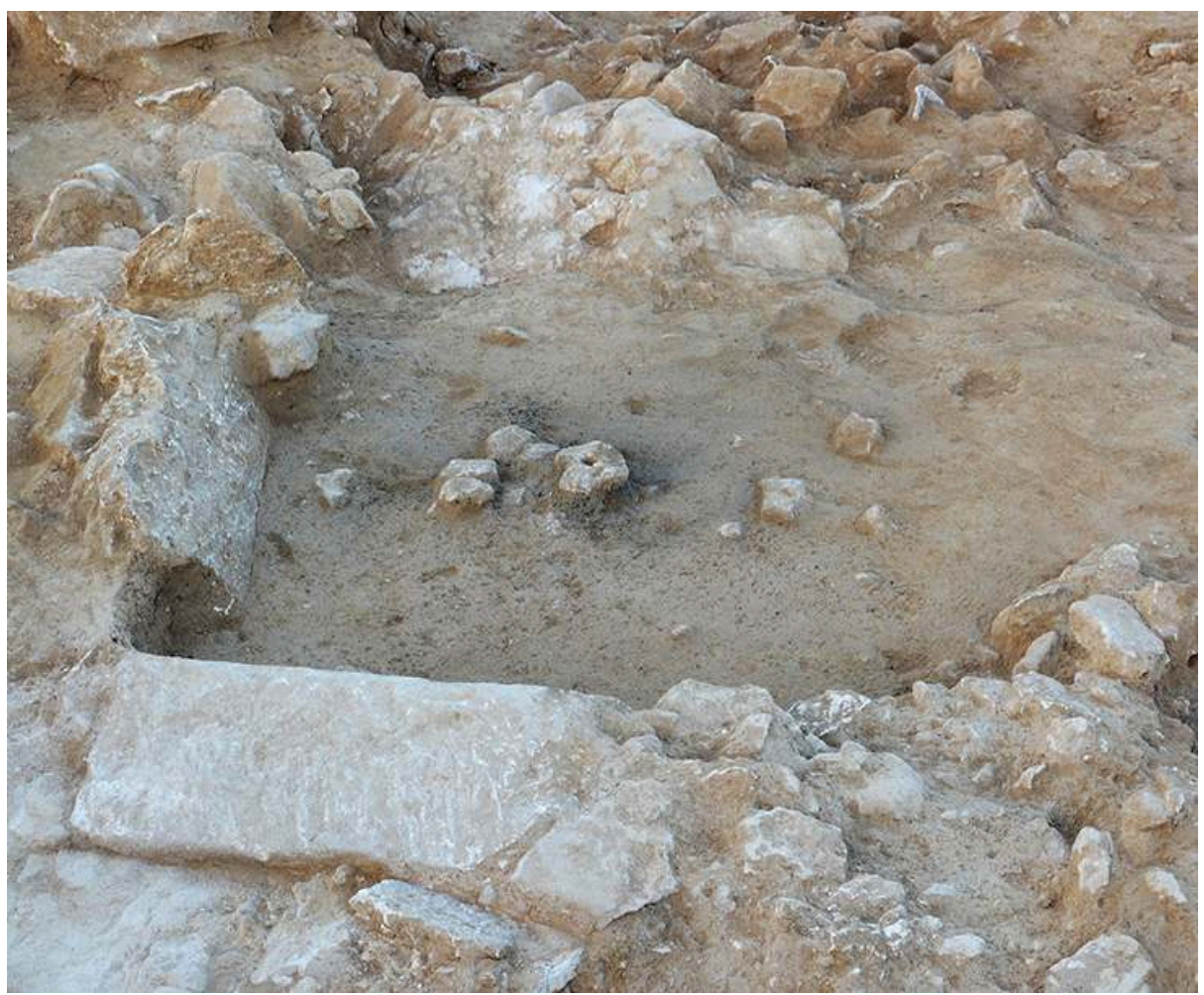

(C) Archives CEAlex. CEAlex_BAH_2020_NDMPF_007

La fouille du niveau 20067 a été effectuée au cours de cette campagne, révélant la présence de plusieurs aménagements (fig. 13-14). Un premier tronçon de dallage (20075 - fig. 15), situé directement au nord du dallage 20014 tout en s'appuyant contre celui-ci et conservé sur une longueur de $2 \mathrm{~m}$ et une largeur de 1,60 $\mathrm{m}$, a ainsi été dégagé. Composé de dalles calcaires en remploi, de débris calcaires et de mortier épais, il correspond à une phase de réfection postérieure au démontage de la partie nord de 20014. Le dallage 20075 est coupé, à l'ouest, par la fosse 20074.

En poursuivant vers le nord et dans l'axe de 20075, un second tronçon de dallage d'orientation E-W (20078 - fig. 15) a été mis au jour. Vraisemblablement contemporain de 20075 , il se compose de dalles de calcaire blanc, dont certaines présentent des encoches, et de fragments de marbre en remploi $(1,60 \times 2 \mathrm{~m})$. 
Fig. 13. Kôm Bahig. Sondage 20000 après dégagement du niveau de démolition 20067 (G. Soukiassian).

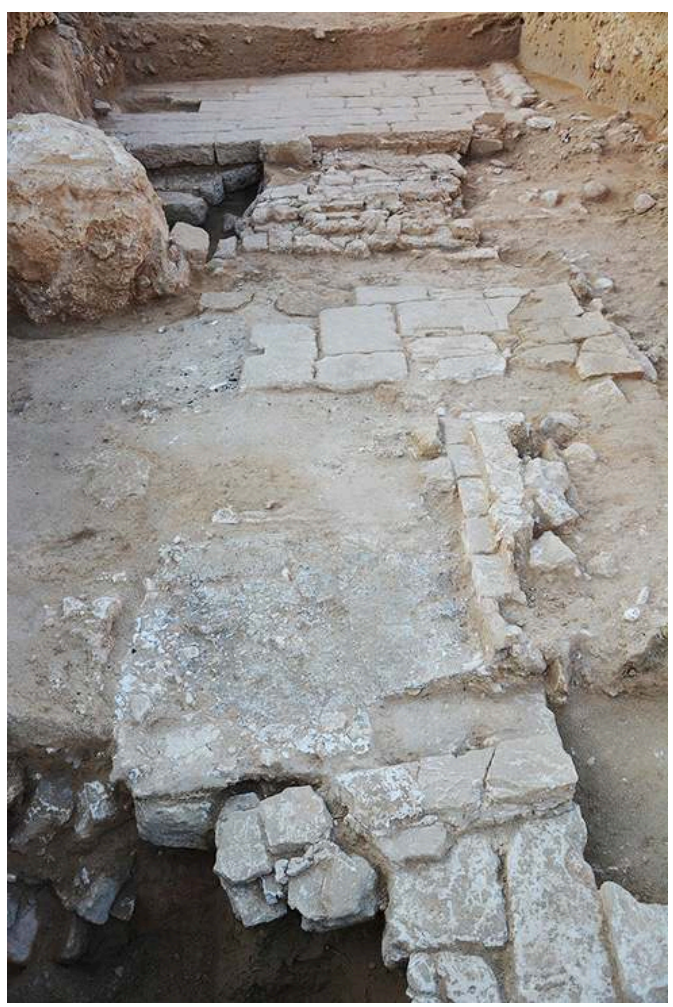

(c) Archives CEAlex. CEAlex_BAH_2020_NDMPF_008 
Fig. 14. Kôm Bahig. Sondage 20000 après dégagement du niveau de démolition 20067 (K. Machinek, G. Soukiassian).

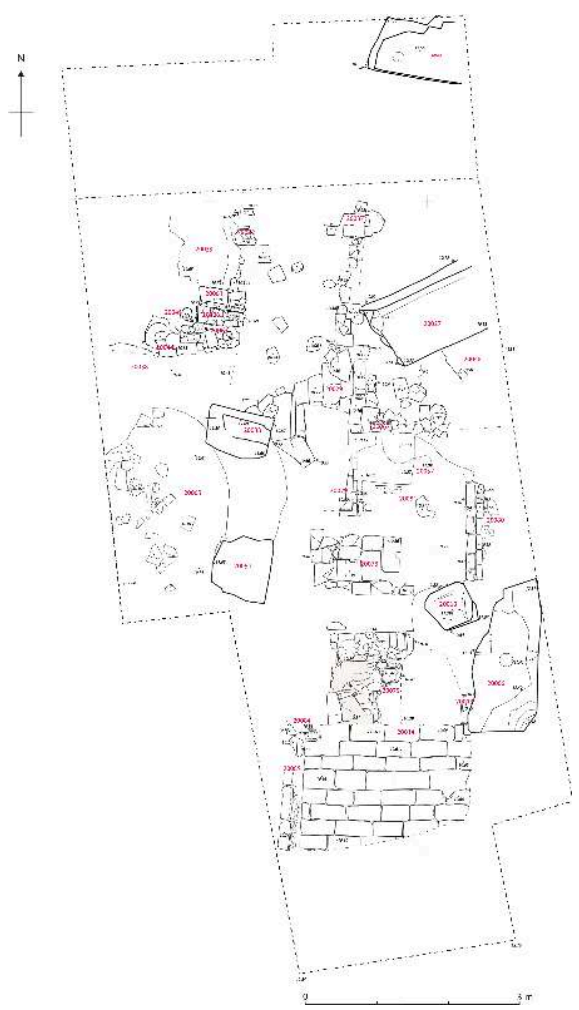

(C) Archives CEAlex. CEAlex_BAH_2020_NDMCN_006 
Fig. 15. Kôm Bahig. Tronçons de dallage 20075 et 20078 au nord de 20014, vue depuis le sud (G. Soukiassian).

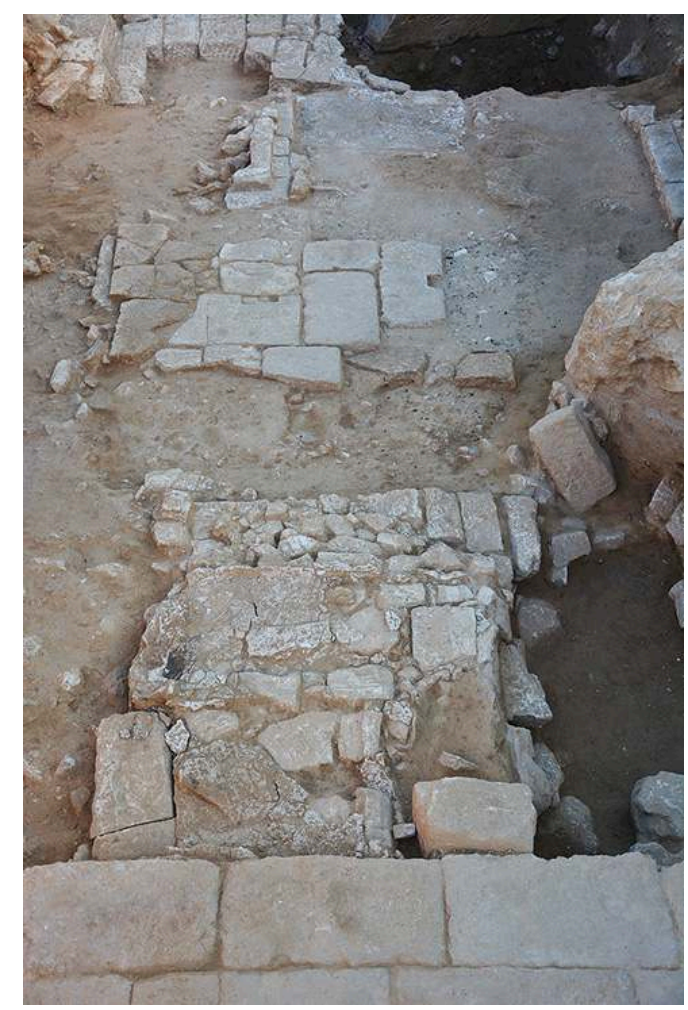

(C) Archives CEAlex. CEAlex_BAH_2020_NDMPF_009

Plus au nord et dans le même alignement que 20075 et 20078, les vestiges d'une bordure calcaire (20079), qui pourrait être un fragment de socle, ont été mis au jour (fig. 16). De même, en limite est du sondage, une installation composée de dalles et blocs calcaires formant les degrés d'une marche (20080) a été découverte (fig. 17). Toujours dans la partie est, un niveau de sol en terre battue mêlée à du charbon, des cailloutis et des déchets de taille (20081), a été atteint. Ce niveau vient buter contre le dallage 20078, la bordure 20079 et l'installation 20080. 
Fig. 16. Kôm Bahig. Bordure calcaire 20079, vue depuis le nord (G. Soukiassian).

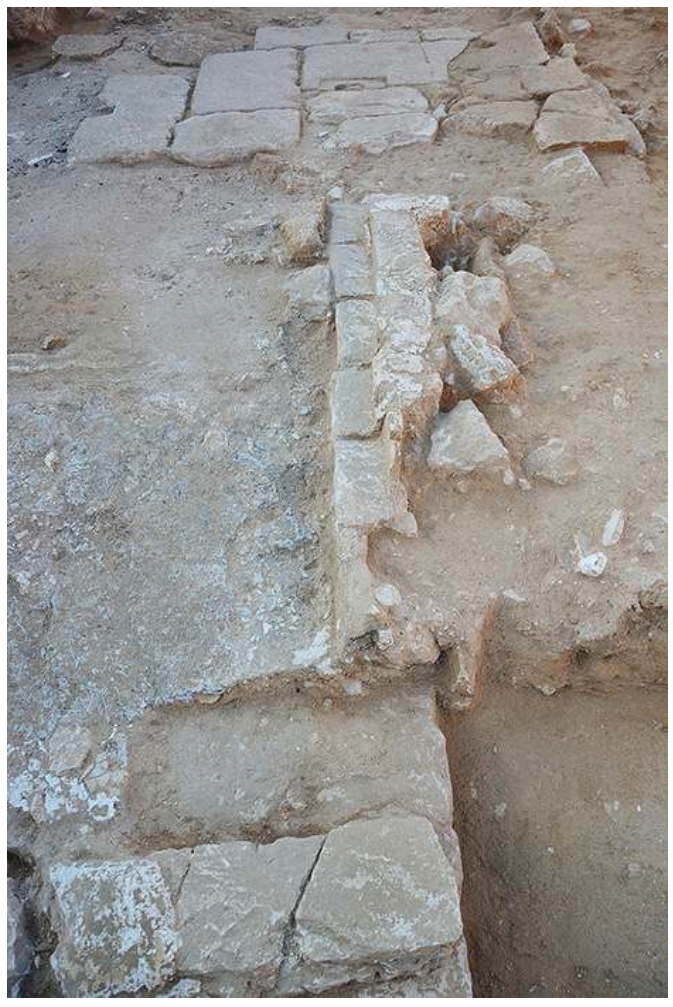

(c) Archives CEAlex. CEAlex_BAH_2020_NDMPF_010

Fig. 17. Kôm Bahig. Détail de l'installation 20080 à l'est du sondage 20000, vue depuis l'ouest (G. Soukiassian).

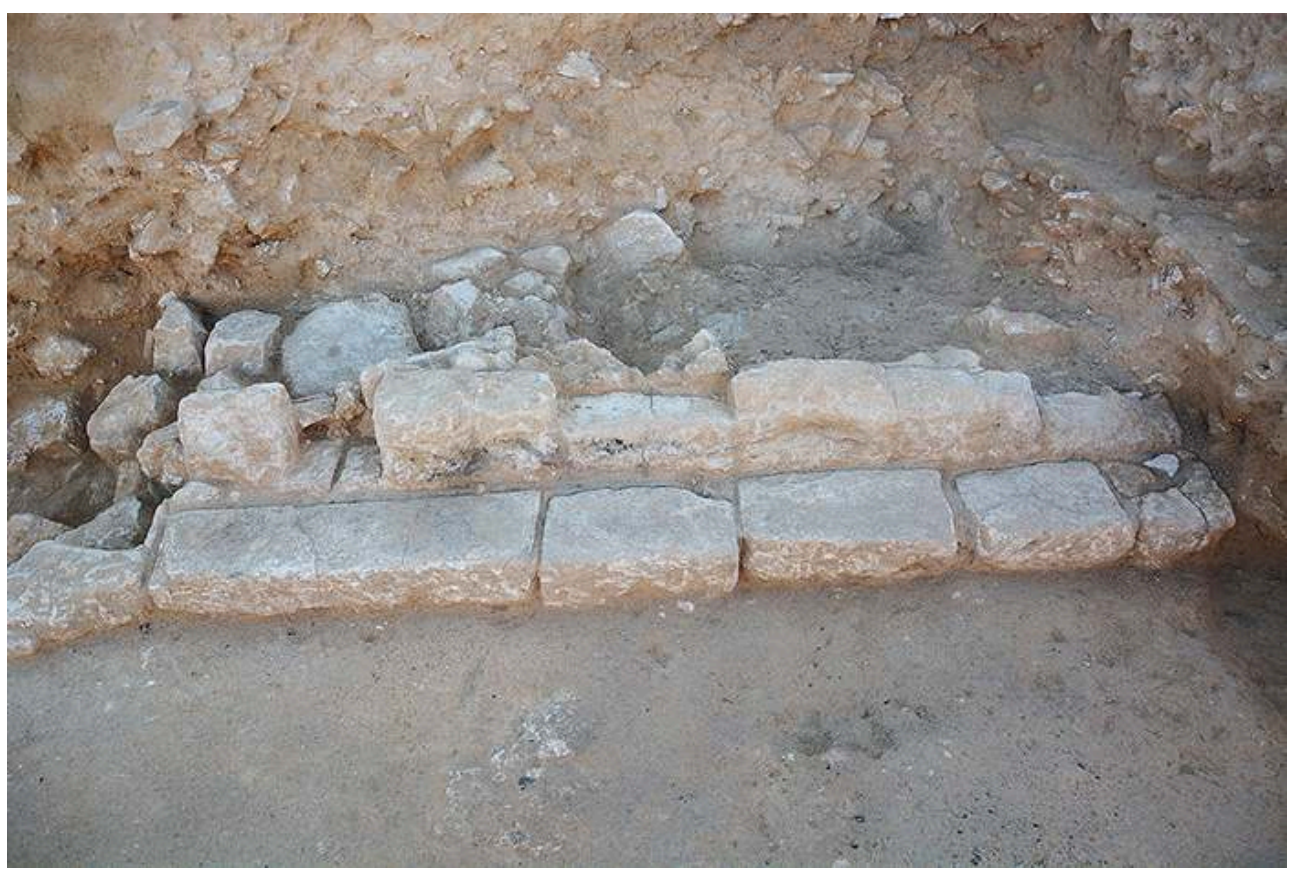

(C) Archives CEAlex. CEAlex_BAH_2020_NDMPF_011

Enfin, les éléments 20075, 20078, 20079, 20080 reposent sur le remblai de nivellement 20082 composé de sable, de déchets de taille de nature calcaire mêlés à de 
l'argile. Ce même niveau avait déjà été identifié dans la partie nord du sondage sous les fragments du dallage en remploi 20037 apparaissant ponctuellement au-dessus du dallage 20029 .

\subsubsection{Sondage 20000 nord et nord-ouest (extensions 2020)}

$19 \mathrm{Au}$ nord du sondage, une extension de $3 \mathrm{~m}$ a été réalisée dans la partie nord-est, permettant le dégagement du petit bloc 20073 ainsi que des faces sud et ouest d'un nouveau gros bloc de calcaire (20070). Ce dernier se poursuit en effet sous la berme nord-est du sondage.

Une seconde extension a été réalisée dans les parties ouest et nord-ouest du sondage afin de dégager la face occidentale du bloc 20033, jusqu'alors sis dans la berme. La fouille de cette zone a également permis de poursuivre le dégagement de la couche de démolition 20028 (couvrant le dallage 20029 et l'ensemble de structures découvertes lors de la campagne précédente ${ }^{4}$ ). Au nord et à l'ouest, la couche de démolition 20028 se poursuit sous la berme tandis qu'au sud-ouest, elle vient s'appuyer contre le bloc 20033. Elle reposait au sud-ouest sur une autre couche de démolition (US 20065), constituée de moellons calcaires (fig. 18). La même opération de dépose après relevé, entreposage et documentation de petits blocs que pour la couche 20056 a été effectuée pour la couche 20065 qui contenait 19 moellons calcaires dont une seule dalle. Ces blocs sont plus petits que ceux du lot 20056, avec des longueurs moyennes de $35 \mathrm{~cm}$ et des largeurs de $25 \mathrm{~cm}$ pour des hauteurs de $20 \mathrm{~cm}$.

Enfin, le radier de pierre 20039, qui avait été mis au jour lors de la campagne 2019, a fait cette année l'objet d'un dégagement puis d'un démontage. Ses limites se poursuivent sous la couche de débris calcaires 20028 et sous les bermes nord et ouest du sondage. Le nettoyage de la coupe nord-sud a permis de distinguer sous 20039 les vestiges d'un four (20062) visiblement endommagé par le creusement de la fosse 20053. Cette zone devrait faire l'objet d'une attention toute particulière lors d'une prochaine campagne. 
Fig. 18. Kôm Bahig. Couche de démolition 20065, blocs 20051 et 20033, vue depuis le nord (A. Simony).

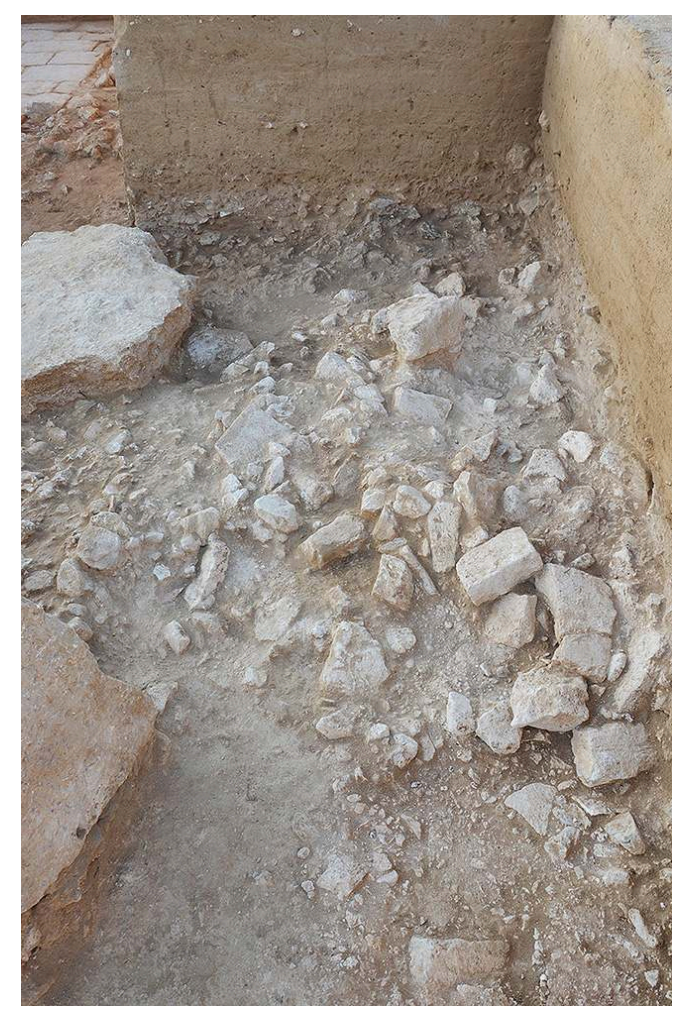

(c) Archives CEAlex. CEAlex_BAH_2020_NDMPF_012

\subsection{Matériel archéologique}

L'ensemble du matériel céramique mis au jour lors de la fouille a été traité en parallèle des travaux de terrain. Le niveau de surface des nouvelles extensions nord et ouest du sondage 20000 (US 20001), de même que les niveaux contenant les gros blocs de calcaire (US 20003, 20004), renfermaient un mobilier céramique très hétérogène couvrant une fourchette chronologique large et composé d'éléments datés de la Basse Époque (amphores de Chios, mortier-cuvette), de l'époque ptolémaïque (amphores de type amphore égyptienne, $\mathrm{AE} 2$, amphores rhodiennes, assiettes à lèvre interne, écuelles, etc.), d'époque romaine (amphores de type $\mathrm{AE} 3$ et $\mathrm{AE} 4$ ) mais également romaine tardive (amphores Late Roman, LRA 1, LRA 4 et sigillées chypriotes $L R$ D) et islamique (un fragment de céramique glaçurée).

L'épaisse couche de débris calcaires couvrant la partie nord du dallage 20029 (US 20028, fig. 19), le sol d'occupation 20052 ainsi que les niveaux de démolition 20056, 20057, 20065, 20066 et 20067 ont livré un matériel céramique assez similaire. Celui-ci est composé d'éléments datés des périodes ptolémaïque (amphores $\mathrm{AE} 2$, jarres torpedo, assiettes à lèvre interne), romaine et romaine tardive (amphores $\mathrm{AE} 3, \mathrm{AE} 4$, Mau XXVII/XXVIII, cratère égyptien d'imitation chypriote marmites à bord évasé, coupelles carénées, amphores LRA 1 et LRA 4, gargoulette à décor peint). La présence de ces éléments tardifs permet de fixer un terminus post quem pour la phase de destruction de la zone. D'autre part, la présence de nombreux fragments architecturaux inscrits ou décorés ainsi que de fragments de statuaire en granit noir au sein de la couche 20067, 
semble résulter du débitage de petits édifices ou installations cultuels qui se trouvaient auparavant dans ce secteur (fig. 20).

Fig. 19. Kôm Bahig. US 20028. Céramiques datées du Haut-Empire. 1-3. Marmites. 4-5. Cratères (A. El Nagar, H. Aboud, C. Edwar).

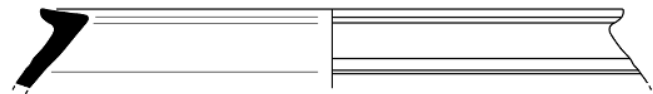

1

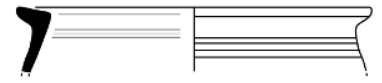

2

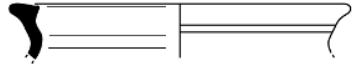

3

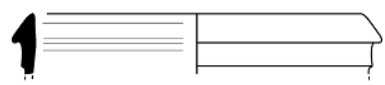

4

Echelle 1/3

(c) Archives CEAlex. CEAlex_BAH_2020_NDMDM_001

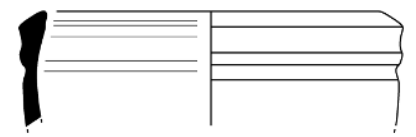

5 
Fig. 20. Kôm Bahig. Fragments architecturaux provenant de la fouille du sondage 20000 (sélection) (P. Soubias).
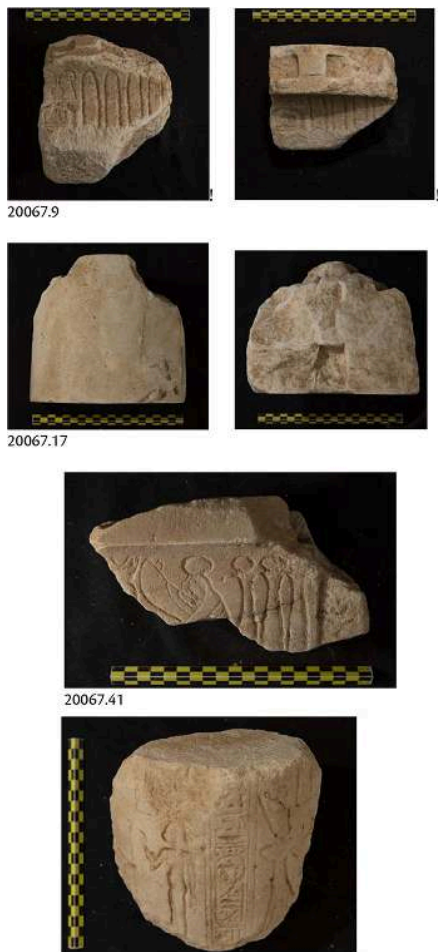

20067.36

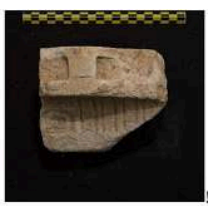

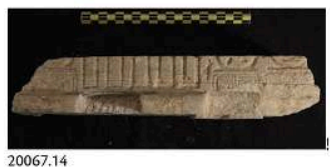

20067.14
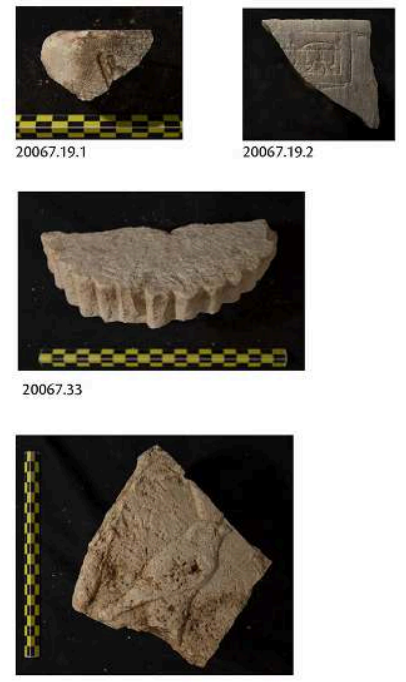

20067.37

(C) Archives CEAlex. CEAlex_BAH_2020_NDMPM_001

24

Le matériel céramique provenant du démontage du radier 20039 (fig. 21) était, à l'exception de quelques fragments vraisemblablement résiduels datés d'époque ptolémaïque, exclusivement daté du Haut-Empire romain (amphores AE 3, AE 3T et $\mathrm{AE} 4$, amphore crétoise, Mau XXVII/XXVIII, cratère d'imitation Paphos 40, jatte à marli, coupe carénée).

Enfin, quelques monnaies ont été découvertes au cours de la fouille. Elles se trouvaient principalement dans les niveaux supérieurs du sondage (US 20003) et le niveau d'éboulis 20065. Une monnaie a également été mise au jour sur le radier 20039. L'ensemble des monnaies a été transféré vers le magasin de fouilles de Tabyiet Nahassin en vue de leur restauration. 
Fig. 21. Kôm Bahig. US 20039. Céramiques datées du Haut-Empire. 1. Amphore AE 4. 2. Cratère. 3. Coupe carénée. 4. Coupe à marli. 5 . Vase à liquides (A. El Nagar, H. Aboud, C. Edwar).

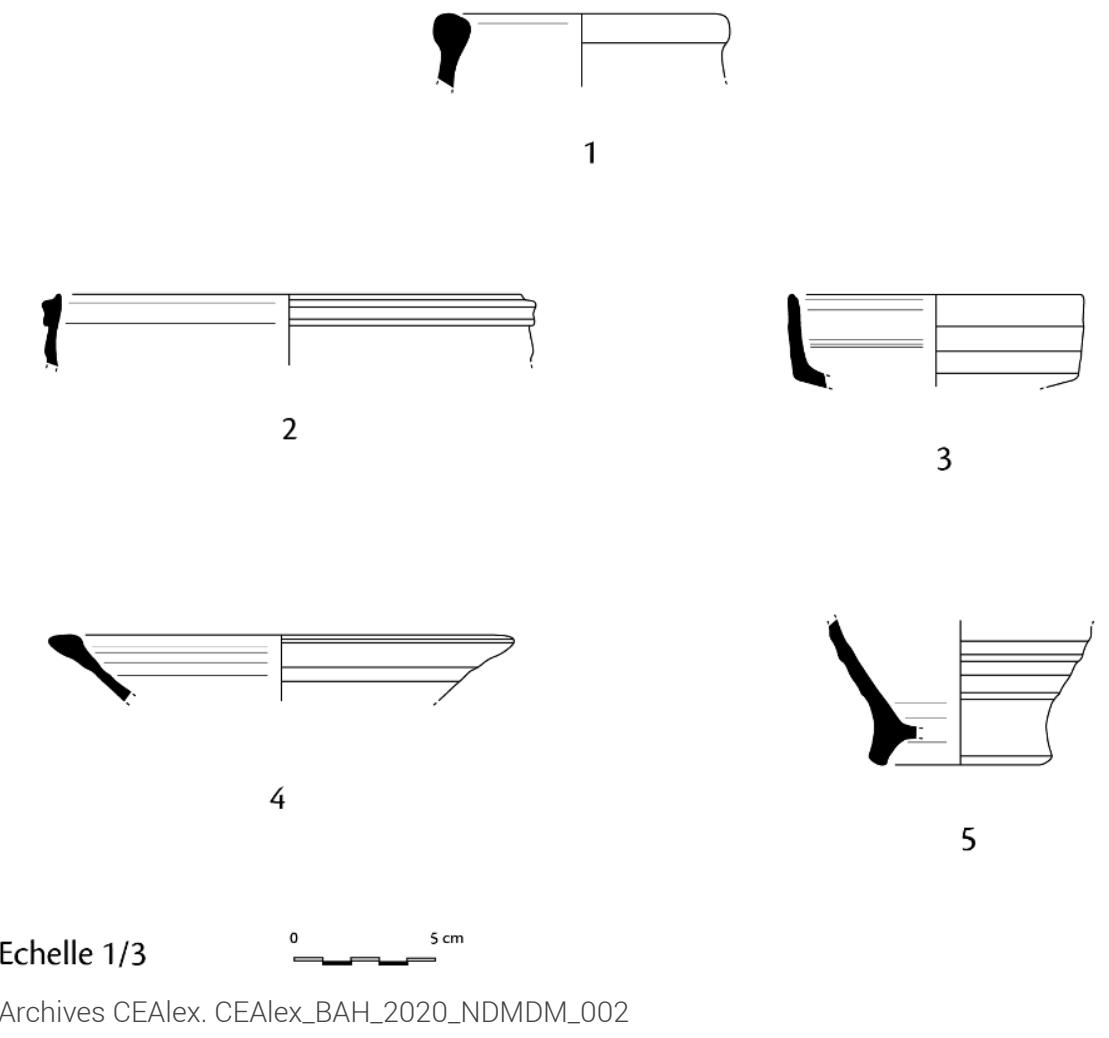

\subsection{Proposition de phasage}

Compte tenu des derniers travaux menés sur le site et des éléments dont nous disposons, il est déjà possible de dégager les grandes phases d'occupation du secteur 20000 :

- La première correspond à l'installation du mur de briques crues 20047 aperçu dans la partie nord-est du sondage.

- La seconde, après l'arasement de ce mur, à l'installation et au fonctionnement des dallages du dromos (dallage 20014 et le sous-dallage 20022 au sud), et du parvis (dallage 20029 au nord). La datation de ces différents éléments n'a pas encore pu être déterminée avec précision. En revanche, il semblerait que leur destruction remonte à la fin de la période ptolémaïque.

- Une troisième phase liée à la réoccupation et la réfection de la zone, correspondant à l'ensemble de structures dégagées en 2019 dans la partie nord-ouest du sondage (le four 20044, le foyer 20045, les niveaux cendreux 20040 et 20041, la structure de briques 20036 et les blocs de calcaire 20061) ainsi qu'aux installations situées au nord du dallage 20014 et dégagées lors de cette campagne (les dallages 20075 et 20078, la bordure calcaire 20079, les installations 20080, 20084, 20085 et le sol 20081).

- Vient ensuite une période d'abandon de la zone matérialisée par d'épaisses couches d'accumulation de nature sableuse visibles au sud comme au nord (US 20013 et 20034).

- Puis, une phase caractérisée par des démolitions et illustrée par le creusement des fosses 20071, 20072 et 20074, les couches de débris de démolition 20056, 20057, 20065, 20066, 20067 et 20076, la couche de débris 20028, la tranchée 20038 et la présence des 
gros blocs de calcaire. Cette phase doit vraisemblablement être décomposée en différentes sous-phases que l'étude du contenu des US permettra de préciser.

La campagne 2020, même écourtée, a offert des résultats importants pour l'établissement de la succession des états de cette zone située à proximité du sanctuaire, où se combinent le dromos et le parvis devant le sanctuaire qui devait se développer vers l'est. Elle a introduit, de manière nouvelle, une phase de réoccupation et de réfection de la zone, avant l'abandon et la phase de récupération des matériaux.

\section{Akadémia}

Valérie Pichot

Responsable d'opération : Valérie Pichot (archéologue, CNRS, CEAlex), assistée par Khaled Moustapha Mohamed Abd El Aziz (contremaître, CEAlex) avec une vingtaine d'ouvriers.

Participants : Mohamed Ahmed Nabil et Samir El Alem (techniciens de fouille), Cécile Shaalan, Ismaël Awad et Ragab Wardani (service de topographie, CEAlex), Philippe Soubias (photographe), Mahmoud Fathy (relevés de terrain), Hisham Aboud (inventaire du mobilier), Ahmed El Nagar (dessins du mobilier). Étude du mobilier amphorique (détermination, tri, comptage, dessin, photographie) par Cansu Yüncüler, Betül Balikçi (université de l'Égée, Izmir) supervisée par Ahmed Kaan șenol (université de l'Égée, Izmir) ; étude du mobilier céramique : Delphine Dixneuf (CNRS, LA3M, UMR 7298). Le MoTA était représenté par Nada Mohamed el-Shatlawy (15 octobre 15 novembre 2021), et par Fatma Mohsen Hassan Mohamed Zalat (16 novembre 17 décembre).

28 Le site d'Akadémia est situé sur la rive méridionale du lac Mariout à une quarantaine de kilomètres au sud-ouest d'Alexandrie et à $1 \mathrm{~km}$ au sud du site de la presqu'île de Maréa (WGS84 latitude: $30.987627^{\circ} \mathrm{N}$; longitude $29.672591^{\circ} \mathrm{E}$ ) (voir fig. 1). Il se compose de deux ensembles connus sous les noms de terrain de l'Académie El-Sadat (à l'ouest) et de terrain de l'université d'Alexandrie (à l'est) et s'étend en contrebas au nord de la ride III, sur à peu près $400 \mathrm{~m}$ le long du canal qui passe au nord du village d'Huwariya.

Ce site comprend deux grandes parties distinctes : à l'ouest deux grandes structures hydrauliques tardives des $\mathrm{v}^{\mathrm{e}}-\mathrm{VI}^{\mathrm{e}} \mathrm{s}$. apr. J.-C. ; à l'est un atelier de production d'amphores vinaires ${ }^{5}$ et un pressoir à vin datés de la fin du $\mathrm{I}^{\mathrm{er}}$-II ${ }^{\mathrm{e}} \mathrm{s}$. apr. J.-C. rattachés à une villa agricole qui a aujourd'hui disparu. Plusieurs secteurs ont été implantés lors de la fouille qui a commencé en 2012 (fig. 22) et s'est achevée en 2020 (campagne du 15 octobre au 17 décembre). 
Fig. 22. Akadémia. Plan topographique (service de topographie du CEAlex).

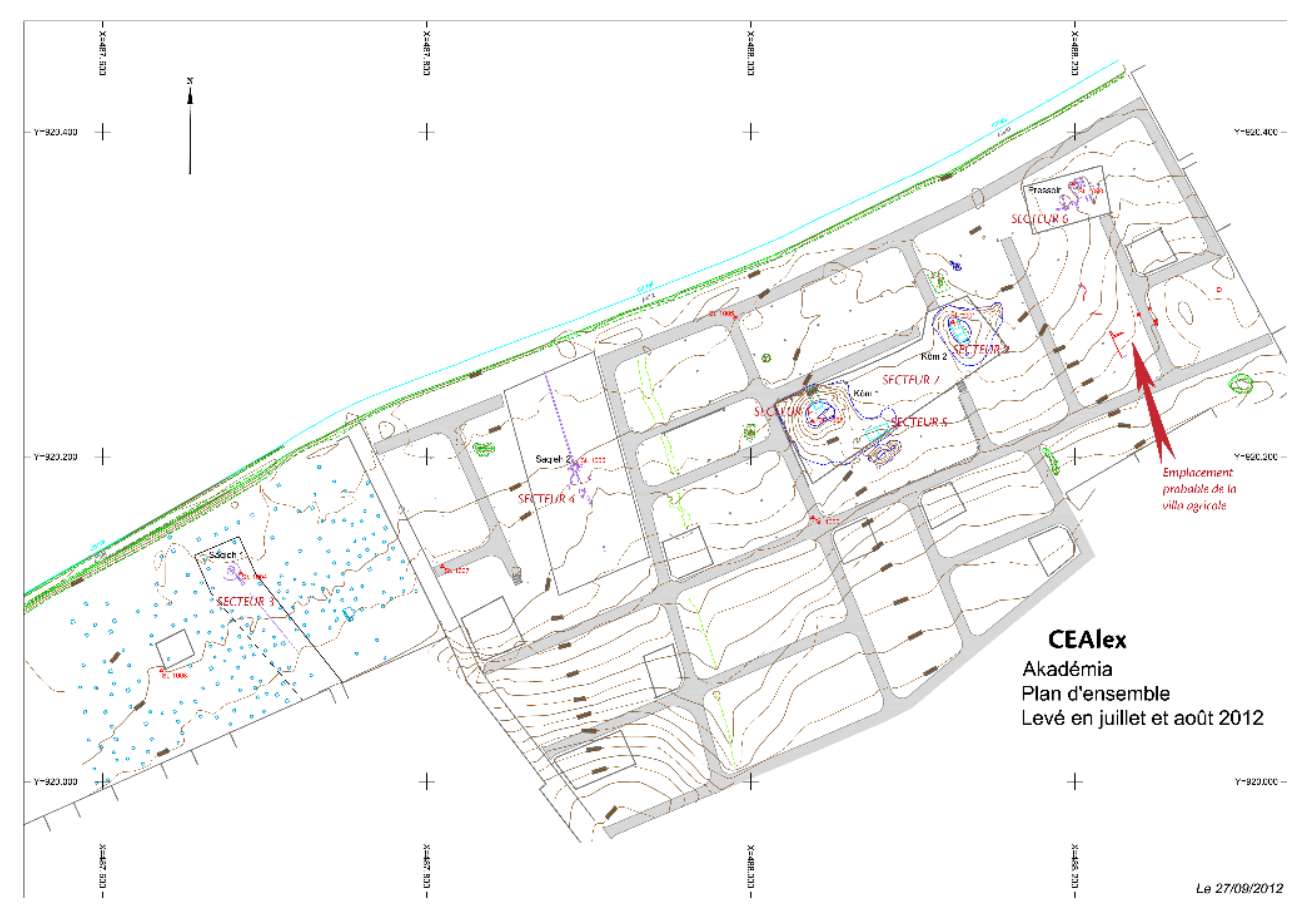

(C) Archives CEAlex. CEAlex_AKA_2020_NDMCN_001

Lartie occidentale comportant les structures hydrauliques et leur environnement a été décomposée en deux secteurs : le secteur 3 qui correspond à la zone de la structure hydraulique occidentale (missions 2012 à 2015) et le secteur 4 à la zone de la structure hydraulique orientale (missions 2012, 2014).

La partie orientale correspondant aux éléments appartenant à la villa agricole a été décomposée en cinq secteurs. Le secteur 6 correspond au pressoir situé au nord-est du site (missions 2014, 2016). La partie conservée et accessible de l'atelier de production d'amphores se situe dans une parcelle rectangulaire de $140 \mathrm{~m}$ est-ouest sur $40 \mathrm{~m}$ nordsud délimitée à l'est et à l'ouest par deux kôms dépotoirs. Le secteur 1 correspond au grand kôm dépotoir occidental et a fait l'objet d'une tranchée de $5 \mathrm{~m}$ sur $13 \mathrm{~m}$ implantée dans sa partie nord depuis le sommet du kôm jusqu'à sa base (missions 2012-2013, 2016) ainsi que d'une fouille de sa partie nord-est (missions 2016 et 2020). Le secteur 2 correspond au kôm dépotoir oriental et a fait l'objet d'un sondage limité à son sommet (mission 2012). Le secteur 5 correspond au petit kôm dépotoir situé au sud-est du secteur 1 et a été fouillé partiellement livrant les vestiges mal conservés d'un four à amphores et des restes de niveaux d'activité postérieurs (missions 2012-2013). Enfin le secteur 7 comprend la partie basse située entre le secteur 1 et 2 , zone relativement plane d'à peu près de $70 \mathrm{~m}$ de long (surface d'à peu près $2800 \mathrm{~m}^{2}$ ) où ont été mis à jour des fours à amphores et de nombreux niveaux d'activité en lien avec la production d'amphores ainsi que quelques niveaux d'activité domestique (missions 2014, 2016 à 2020). Les nombreux vestiges découverts sur cette partie du site n'appartiennent pas tous à la même phase d'occupation, l'atelier ayant évolué au cours du temps ${ }^{6}$, mais ils permettent d'illustrer les différentes phases de la chaîne opératoire de la production d'amphores, de la préparation de l'argile au poissage des amphores. 

Nicosie (Chypre) en avril 2013 et à deux articles, le premier dans les actes de ce colloque (à paraitre), le second dans le BCE $24^{7}$. Les résultats de la mission de 2014 ont, quant à eux, fait l'objet de deux articles dans le $B C E 25^{8}$. Un article illustrant la chaîne opératoire de la production d'amphores vinaires, de l'extraction de l'argile au poissage des amphores, va être publié dans le prochain Delta Survey Workshop of Egypt Exploration Society and Ministry of Antiquities. (archéologie ${ }^{10}$, géomorphologie ${ }^{11}$, étude du mobilier ${ }^{12}$, études chimique ${ }^{13}$ et pétrographique ${ }^{14}$ de la production de l'atelier) sous la forme d'un volume dans les Études Alexandrines est en préparation et prévu à moyen terme.

L'objectif de la campagne 2020 était d'une part de terminer l'exploration du secteur 7 situé entre les kôms dépotoirs des secteurs 1 et 2 , et occupé principalement par un atelier de production d'amphores, et d'autre part d'explorer l'accès à la chambre de chauffe du four occidental mis en évidence dans le secteur 1 (sondage 3) lors de la mission de $2016^{15}$.

\subsection{Secteur 1 , le four occidental}

En 2016, le sondage 3 implanté dans la partie nord-est du secteur 1 (fig. 23) avait livré en partie sous le grand kôm de rejet les vestiges de deux grands fours de même type que le four oriental mis au jour dans le secteur 7. Les restes du four le plus ancien (en pointillé vert sur la fig. 23) situés dans la partie nord-ouest étaient peu lisibles, le four ayant été presque entièrement détruit par l'implantation d'une part, d'un autre four plus récent et d'autre part, du grand mur est/ouest qui traverse l'atelier de part en part (en grisé sur fig. 23). Les vestiges du four plus récent (en rose sur la fig. 23) détecté dans la partie sud et orientale du sondage 3 étaient très bien conservés. Nous avions pu mettre en évidence la sole du four, le mur circulaire en briques crues de $2 \mathrm{~m}$ d'épaisseur (rubéfié à proximité de l'intérieur du four), une série de couches imposantes de maintien des fondations du four, la limite occidentale de la fosse de chargement (au nord du four) composé d'un massif de briques crues (partie supérieure) et de pierres calcaires (partie inférieure). Ces vestiges correspondaient à la partie nordouest du grand four déjà mis en évidence en 2014 dans le carré 3 du secteur 7 . Les dimensions du four avaient pu être estimées plus précisément : aux environs de $14 \mathrm{~m}$ de diamètre extérieur et de $8,5 \mathrm{~m}$ de diamètre intérieur, ce qui en fait le plus grand four d'amphores connu aujourd'hui en Maréotide ${ }^{16}$.

Malgré l'impossibilité d'explorer la partie inférieure du four à cause de la proximité de la nappe phréatique (altitude $4,10 \mathrm{~m})^{17}$, nous savons néanmoins que la substructure descend profondément grâce à l'étude effectuée sur le four oriental du secteur $7:$ le fond de la chambre de chauffe, les niveaux d'activité dans la fosse de chargement et l'alandier sont estimés à environ $4 \mathrm{~m}$ en dessous du niveau inférieur de la sole, c'est-àdire à environ $0,45 \mathrm{~m}$ d'altitude. Nous connaissons aussi l'organisation générale de la structure interne de la chambre de chauffe de ce type de four grâce au four de Borg elArab et à celui de « Maréa »-Philoxénité (fig. 24). 
Fig. 23. Akadémia. Plan topographique, secteur 1 , sondage 3 et secteur 7 (service de topographie du CEAlex).

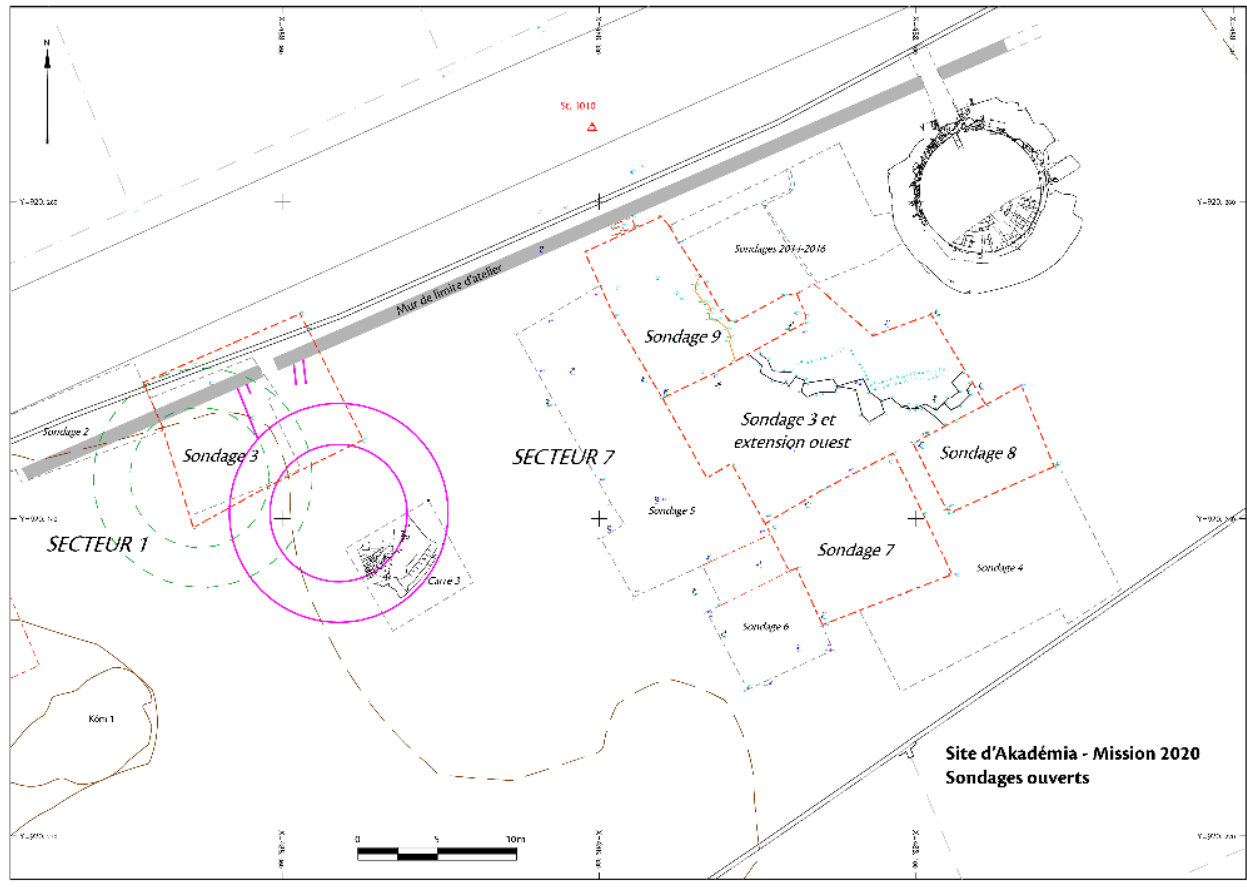

(c) Archives CEAlex. CEAlex_AKA_2020_NDMCN_002

Fig. 24. Restitution d'un four à amphores de Maréotide d'après les données issues de l'étude du four oriental d'Akadémia et des parallèles de Maréotide (V. Pichot, M. Abdelaziz).

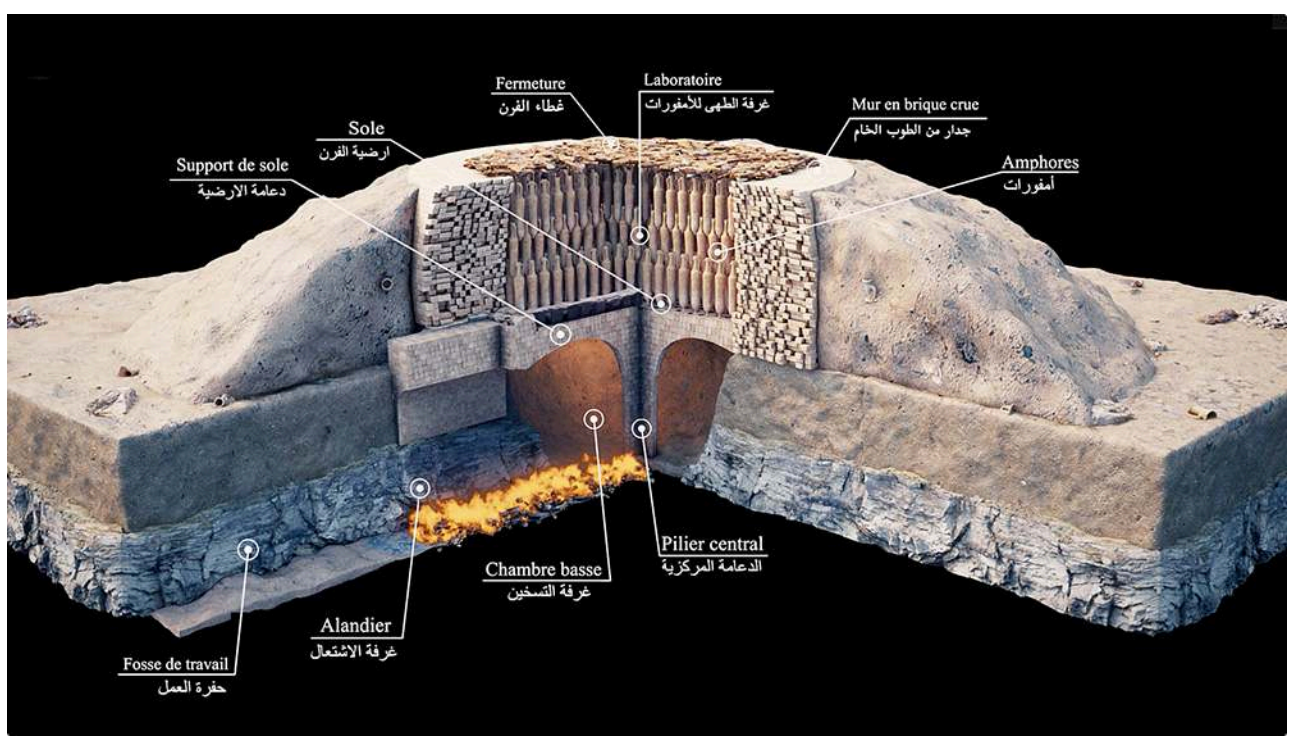

(c) Archives CEAlex. CEAlex_AKA_2020_NDMCN_003

\subsubsection{Secteur 1 , sondage 3 , extension orientale}

Le sondage 3, initialement de 7,7 m (nord/sud) sur 7,1 m (est/ouest), a été étendu vers l'est sur $4,5 \mathrm{~m}$ et au nord sur un peu moins de $2 \mathrm{~m}$ afin de récolter des informations sur 
les aménagements (descenderie et fosse de chargement du combustible) donnant accès à l'alandier et à la chambre de chauffe.

Dans la partie étendue du sondage, le four occidental apparaît sous le niveau d'abandon du site (formation éolienne, US 10104) et sous une couche peu épaisse composée d'un sédiment argileux jaune mélangé avec quelques fragments d'amphores (US 10105) provenant de la dégradation de la structure avant son enfouissement. L'intérieur du laboratoire est rempli jusqu'à la sole d'une couche formée par la destruction des parois du four et de son muret intérieur (briques rubéfiées et délitées en vrac mêlées à un sédiment légèrement sableux, US 10106). L'accès à l'alandier par une descenderie associée à une fosse de chargement est matérialisé, comme dans le cas du four oriental (secteur 7), par la rupture située dans le mur qui délimite l'atelier au nord (fig. 25).

Fig. 25. Akadémia. Secteur 1, sondage 3 : dégagement de la partie nord du four à amphores (vue depuis l'ouest), l'accès à la chambre de chauffe est matérialisé par la rupture dans le mur de limite nord de l'atelier (V. Pichot).

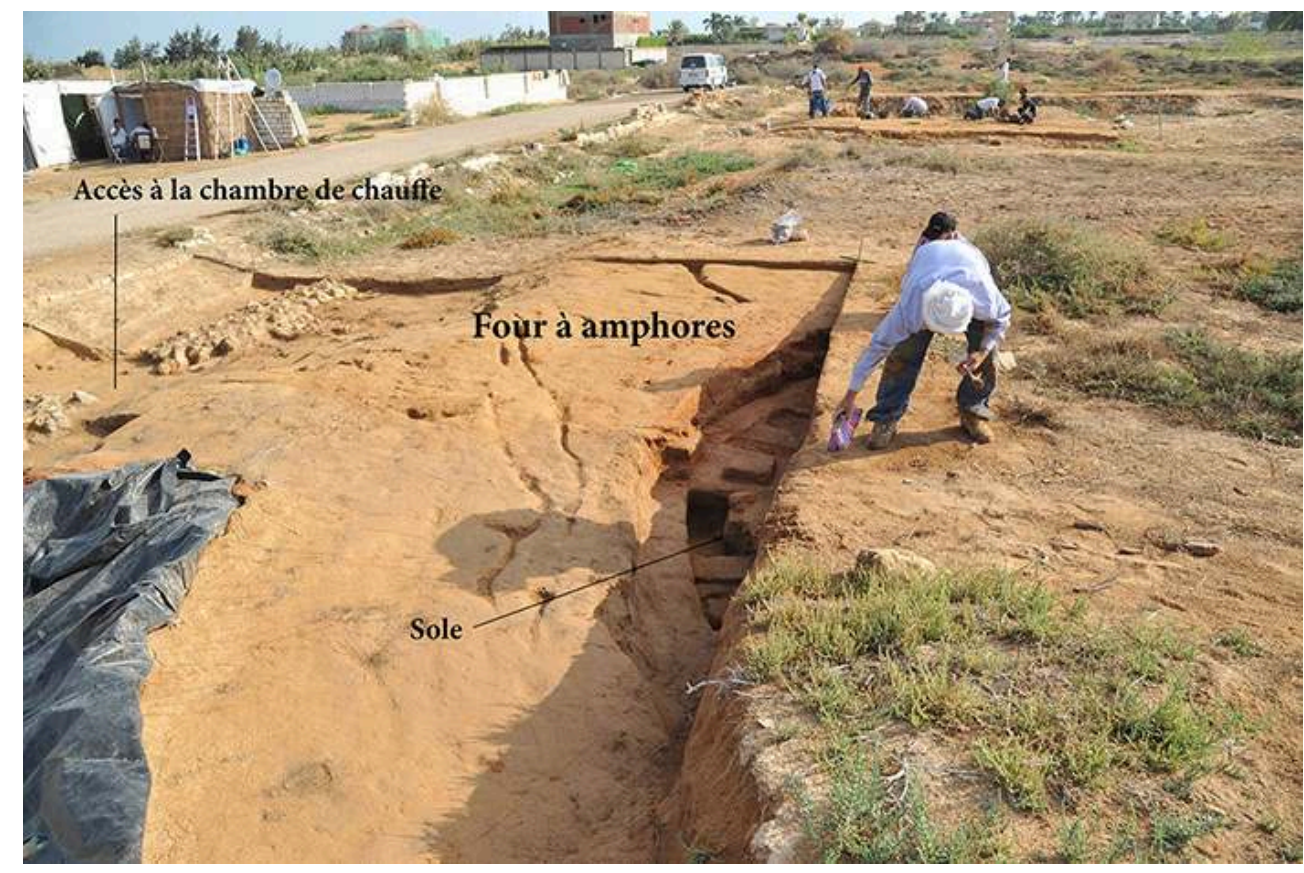

(C) Archives CEAlex. CEAlex_AKA_2020_NDMPF_001

Nous avons procédé au dégagement de la partie nord du four intacte sous des couches liées à l'abandon/destruction de la zone (US 10107, 10108, 10110, 10114) (fig. 26). Certaines couches constructives ont été enlevées afin de mettre en évidence l'organisation mise en œuvre au-dessus de la fosse de chargement: l'US 10109, couche de maintien composée de fragments d'amphores est localisée dans la partie orientale du sondage; les US 10111, 10112, couches argileuses relativement compactes sont installées sur la couverture de la fosse de chargement en éolianite d'une vingtaine de centimètres d'épaisseur maximum (US 10113). 
Fig. 26. Akadémia. Secteur 1 , sondage 3 : partie nord du four à amphores occidental après dégagement, vu depuis le sud-est (V. Pichot).

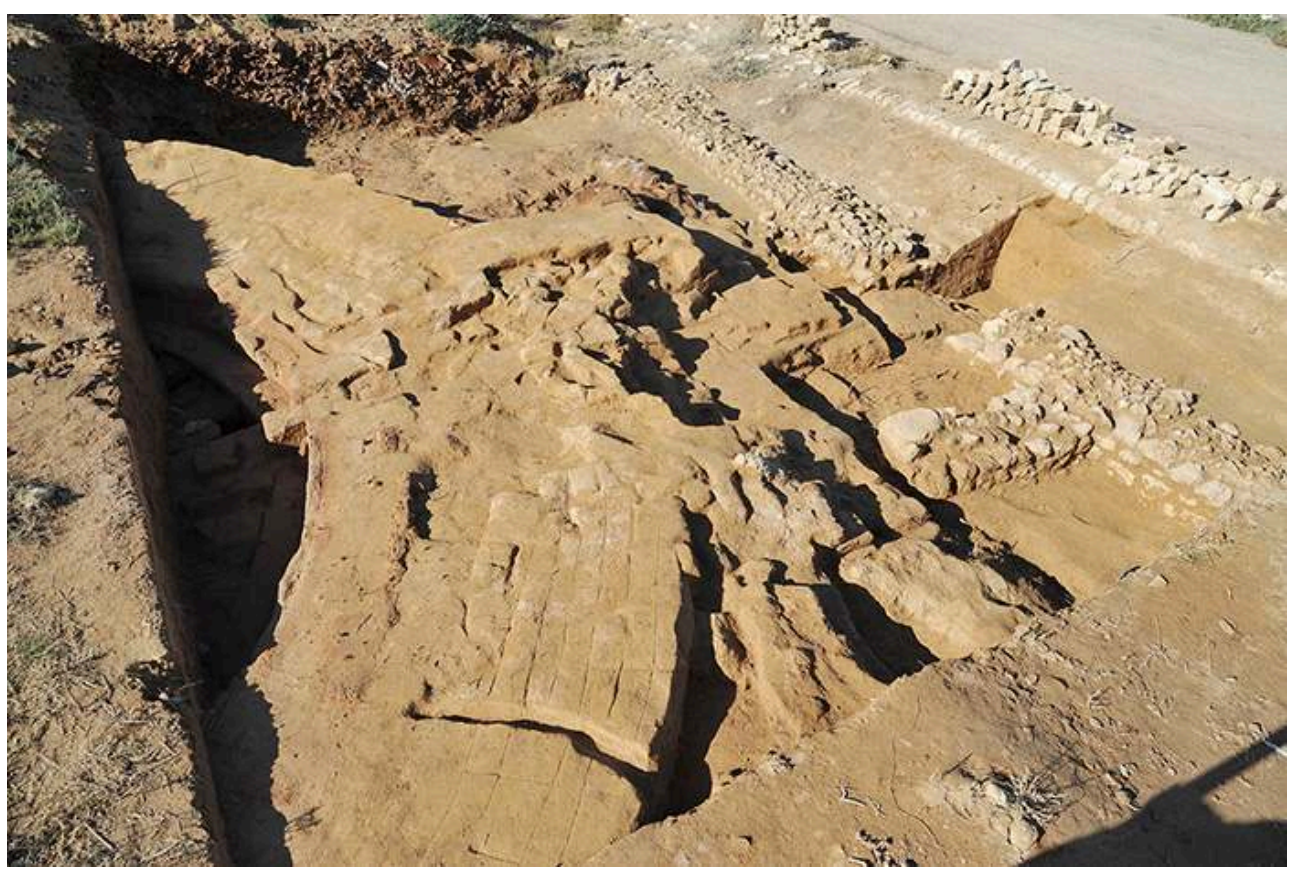

(c) Archives CEAlex. CEAlex_AKA_2020_NDMPF_002

\subsubsection{Fosse de chargement et alandier}

Le traitement des données et l'interprétation de l'ensemble sont en cours de réalisation, mais nous pouvons déjà souligner quelques points (fig. 27-28). Comme pour le four oriental du secteur 7, l'accès à la fosse de chargement et à l'alandier se faisait par le nord, très certainement par une descenderie associée à une partie plane qui faisait la jonction avec la fosse de chargement. Aujourd'hui l'ensemble se trouve sous la route asphaltée et commence peut-être même plus au nord. La rupture $(1,10 \mathrm{~m}$ de largeur) dans le mur de « limite » de l'atelier marque la jonction entre la descenderie et la fosse de chargement : c'est à cet endroit que commence la couverture de la fosse de chargement (altitude supérieure de la couverture $4,61 \mathrm{~m}$; altitude du passage sous la couverture 4,45 m, qui correspond à l'altitude inférieure estimée de la sole).

La fosse de chargement est limitée à l'est et à l'ouest par deux massifs d'environ 0,70 m d'épaisseur constitués de briques crues (partie supérieure) et de pierres calcaires (partie inférieure) qui viennent s'insérer au sud dans le mur circulaire en briques crues du four. Ces massifs débutent de chaque côté de la rupture dans le mur de « limite »; ils ne sont pas tout à fait parallèles : si le massif occidental est orienté nord-ouest - sud-est et fait un angle droit avec le mur de « limite ", le massif oriental est orienté nord-est sud-ouest et fait un angle de $80^{\circ}$ avec le mur de «limite » l'atelier, ce qui fait que la fosse de chargement est plus large au nord (environ 3,30 m) qu'au sud (environ $3 \mathrm{~m}$ ). La longueur nord-sud de la fosse de chargement (entre le mur de «limite» d'atelier et le mur circulaire du four) serait de 3,30 m et la hauteur sous couverture est estimée à $4 \mathrm{~m}$ au maximum (la présence de la nappe phréatique empêche une exploration plus poussée). En outre, on remarque qu'entre les deux massifs en briques crues, au contact $\mathrm{du}$ mur circulaire du four (zone qui se situe au-dessus de la jonction entre la fosse de 
chargement et l'alandier), l'organisation des briques crues est inversée par rapport à celle du mur circulaire du four et forme un arc ouvert vers le nord.

42 C'est la première fois qu'est identifié clairement en fouille cet aménagement qui est partie prenante du four.

Fig. 27. Secteur 1, sondage 3, orthophoto de la partie nord du four en cours de fouille (P. Soubias).

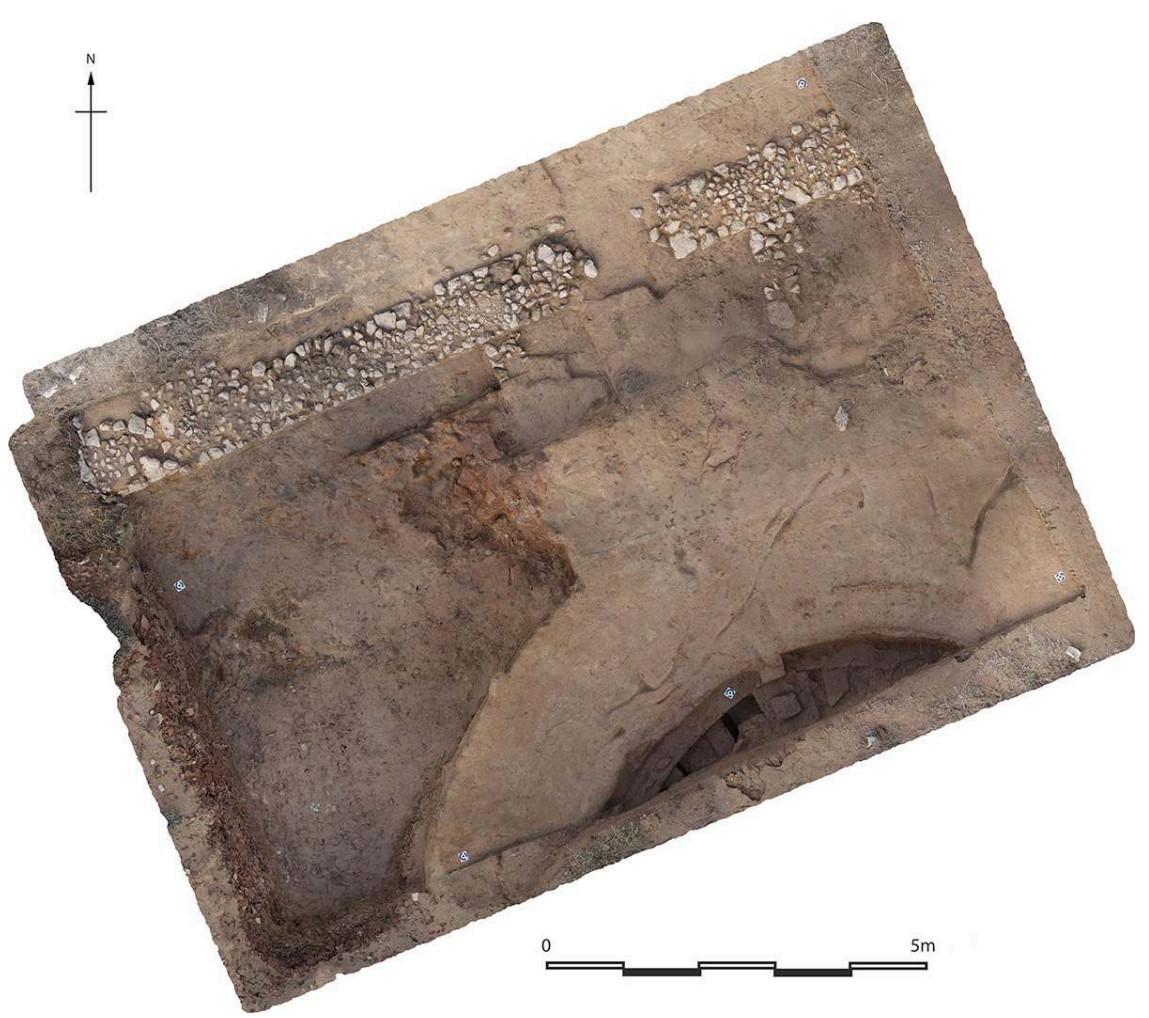

(c) Archives CEAlex. CEAlex_AKA_2020_NDMCN_004

Fig. 28. Akadémia. Secteur 1, sondage 3 : partie nord du four à amphores occidental après dégagement, vu depuis le nord (V. Pichot).

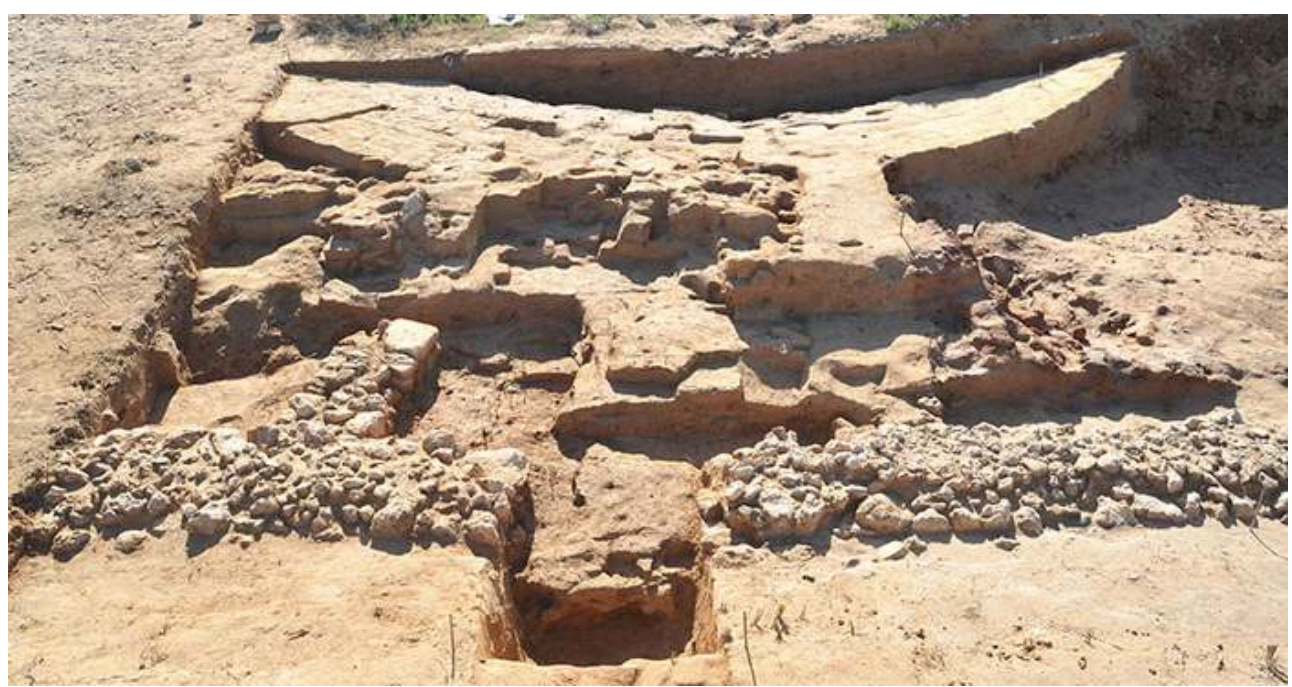

(c) Archives CEAlex. CEAlex_AKA_2020_NDMPF_003 


\subsection{Secteur 7}

Le secteur 7, orienté est-ouest, a pour dimension $60 \mathrm{~m}$ sur $35 \mathrm{~m}$ (voir fig. 23). L'exploration de ce secteur a livré de nombreuses informations sur l'organisation et l'évolution de l'atelier et sur les étapes de la chaîne opératoire de production d'amphores (malaxage de l'argile, façonnage des amphores, cuisson, mise en résine). L'exploration de cet ensemble avait pour objectif de compléter les informations sur l'évolution de l'atelier, afin d'en extraire une chronologie relative et d'acquérir des informations nouvelles sur les différentes activités du secteur, y compris celles de nature domestique présentes sur une partie du secteur dans les dernières phases d'occupation.

\subsubsection{Secteur 7 , sondages 7 et 8}

44 Dans le sondage 7 ( $9,2 \mathrm{~m}$ est/ouest sur $8,4 \mathrm{~m}$ nord/sud) et le sondage 8 ( $7,8 \mathrm{~m}$ est/ouest sur $5,4 \mathrm{~m} \mathrm{nord} / \mathrm{sud}$ ), directement sous la couche liée à l'abandon du site (formation éolienne, US 70213, sondage 7 et US 70215, sondage 8), nous avons mis au jour les vestiges d'un sol en briques crues (US 70253) relativement bien préservé malgré quelques effondrements notés au nord-ouest et un hiatus dans la partie nord-est (fig. 29-30). Il avait été repéré en 2018 dans une tranchée d'1,40 $\mathrm{m}$ sur $5 \mathrm{~m}$ de longueur (nord/sud) implantée entre les sondages 3 et 4 et correspond vraisemblablement au premier sol mis au jour en 2018 plus au sud dans le sondage 4 ainsi qu'à un sol découvert en 2019 plus à l'ouest entre les sondages 5 et 6 .

Le dégagement de ce sol en briques crues a mis en évidence la présence de trous de poteau et piquet ainsi que des négatifs de base d'amphores et de céramiques. La digitalisation des relevés effectués cette année et l'élaboration du plan d'ensemble sont en cours. Couplée à la photogrammétrie (fig. 31), à l'étude des stratigraphies et des profils, l'analyse du plan d'ensemble comportant les différents niveaux de sol permettra de proposer une interprétation de l'organisation de l'occupation et de son évolution sur cette zone du secteur 7 en partie dédiée à des activités domestiques. 
Fig. 29. Akadémia. Secteur 7, au premier plan sondage 7, sol en briques crues (US 70253) dégagé ; à l'arrière-plan sondage 8 , le même sol en briques crues en cours de dégagement, vus depuis l'ouest (entre les deux, tranchée qui avait révélé l'existence du sol lors de sa fouille en 2018). (V. Pichot).

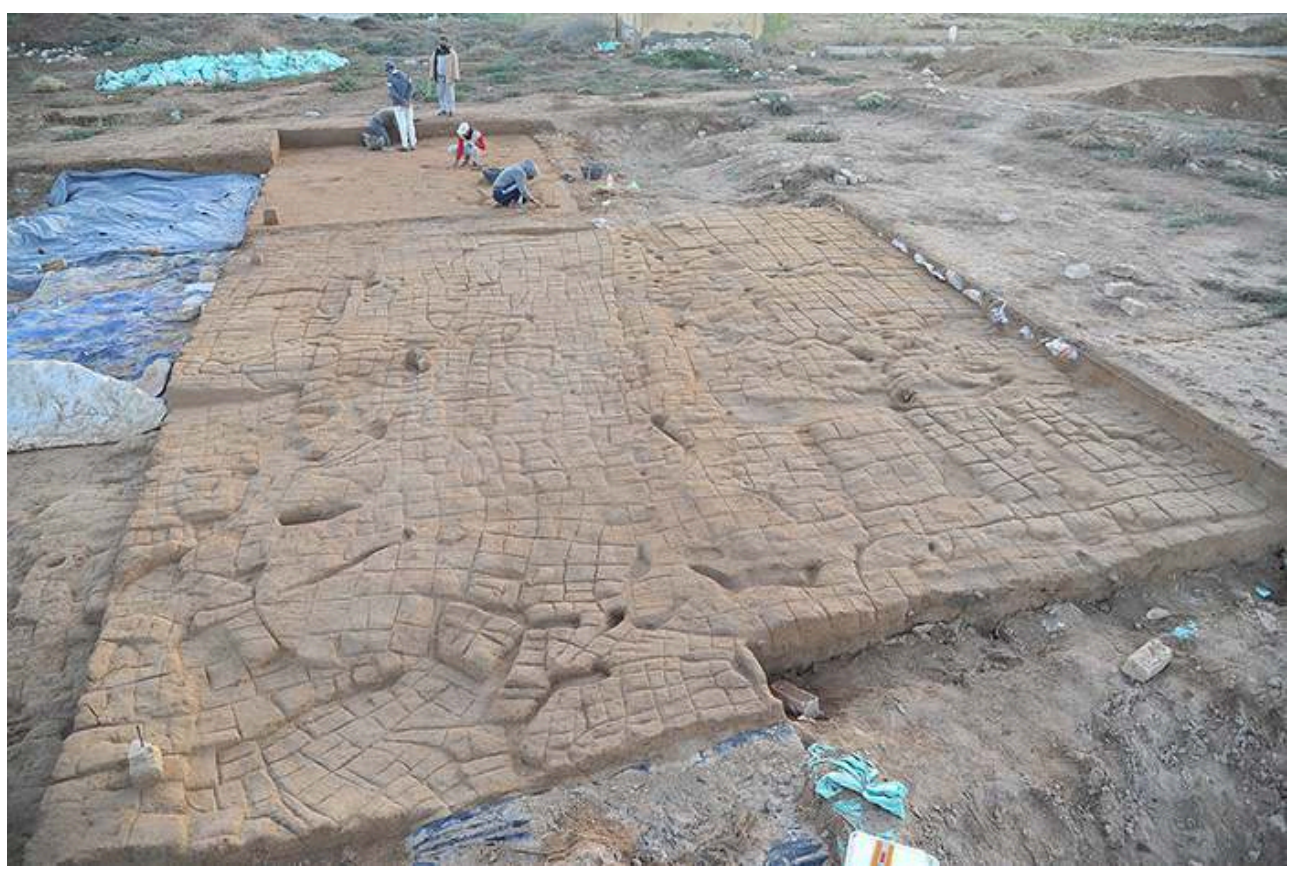

(C) Archives CEAlex. CEAlex_AKA_2020_NDMPF_004

Fig. 30. Akadémia. Secteur 7 , sondages 7 et 8 , sol en briques crues dégagé en cours de relevé, vu depuis l'est (V. Pichot).

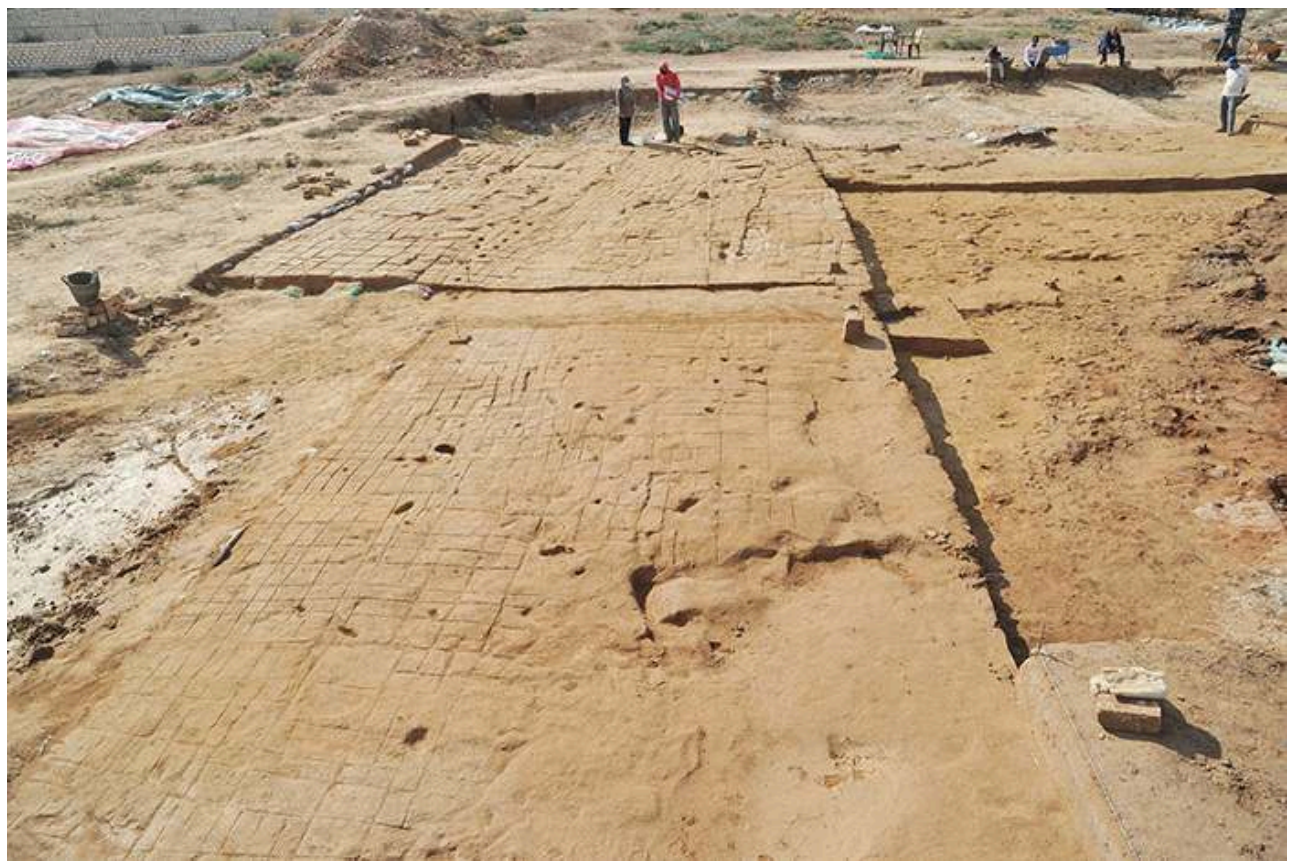

(c) Archives CEAlex. CEAlex_AKA_2020_NDMPF_005 
Fig. 31. Akadémia. Secteur 7, orthophoto du secteur 7 en cours de fouille (P. Soubias).

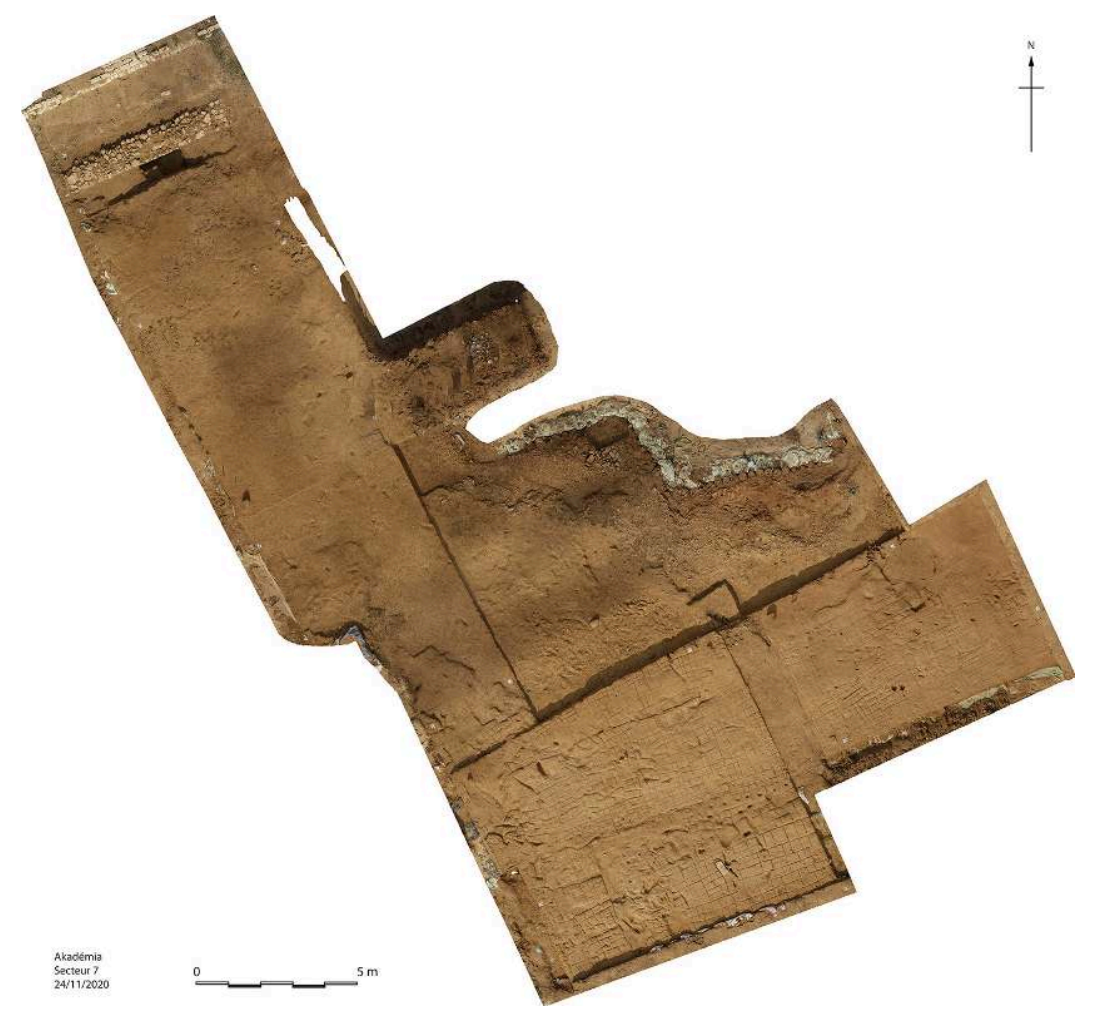

(C) Archives CEAlex. CEAlex_AKA_2020_NDMCN_005

\subsubsection{Sondage 9} ouest sur 2,2 $\mathrm{m}$ nord-sud) est situé dans la partie nord du secteur 7 à environ $12 \mathrm{~m}$ à l'ouest du four oriental. Comme dans les sondages 7 et 8 , un premier niveau de sol en briques crues (US 70216) a été mis au jour directement sous la couche d'abandon du site (formation éolienne, US 70214). Son organisation est peu lisible, les briques crues ayant complètement fondu (fig. 32). Il succède à trois autres niveaux de sol en briques crues qui ont été dégagés durant la mission. 
Fig. 32. Akadémia. Secteur 7 , sondage 9 vu depuis l'ouest, au premier plan sol en briques crues très détérioré (US 70216), au second plan, premier niveau (US 70217) de la succession de couches d'amphores en cours de nettoyage (V. Pichot).

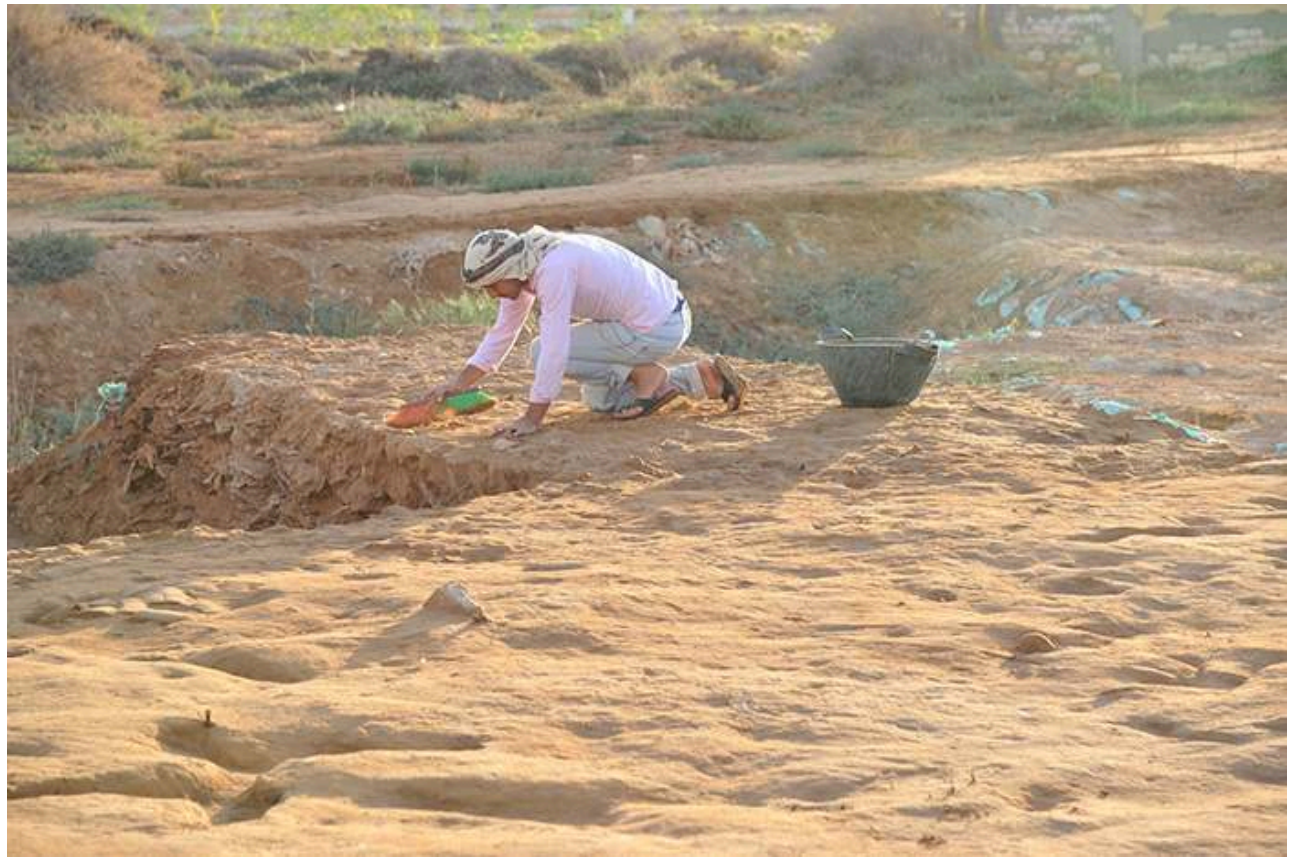

(C) Archives CEAlex. CEAlex_AKA_2020_NDMPF_006

47 Dans le retour sud-est du sondage une succession de couches d'amphores (pendage estouest) est liée à l'installation du four oriental: il s'agit du reliquat de couches participant au maintien du four et fouillées durant les missions 2014 et 2016. L'US 70217 correspond au premier niveau de cette succession de couches et vient remplacer le premier sol en briques crues (US 70216) à l'est, là où ce niveau de sol a été très dégradé, cassé et délité. Nous retrouvons sous ce premier niveau d'amphores quelques restes du sol 70216 mélangé à des amphores incrustées (l'ensemble porte le numéro d'US 70219) provenant de la couche 70217 .

L'ensemble du sondage (excepté le retour au sud-est) est occupé par la succession de sols en briques crues (US 70216, US 70224, US 70218) séparés les uns des autres par des couches de nivellement composées de sédiments argilo-sableux mêlés à des fragments d'amphores (US 70227, US 70228). Si les sols supérieurs (US 70216, US 70224) étaient peu lisibles et très perturbés, le troisième niveau de sol était mieux préservé : il correspond au niveau de sol en briques crues qui se développe vers le sud dans l'extension ouest du sondage 3 (US 70218). Le dégagement de ce sol en briques crues (fig. 33) a mis en évidence la présence de trous de poteau et piquet ainsi que des négatifs de base d'amphores et de céramiques. On note dans la partie nord une zone restreinte où les sols en briques crues ont été cassés et des fragments d'amphores en vrac ont remplacé les sols (US 70226, US 70231, US 70242). 
Fig. 33. Akadémia. Secteur 7 , sondage 9 vu depuis le nord, troisième sol en briques crues (US 70218) en cours de dégagement, au premier plan rupture dans le sol et US 70242 (V. Pichot).

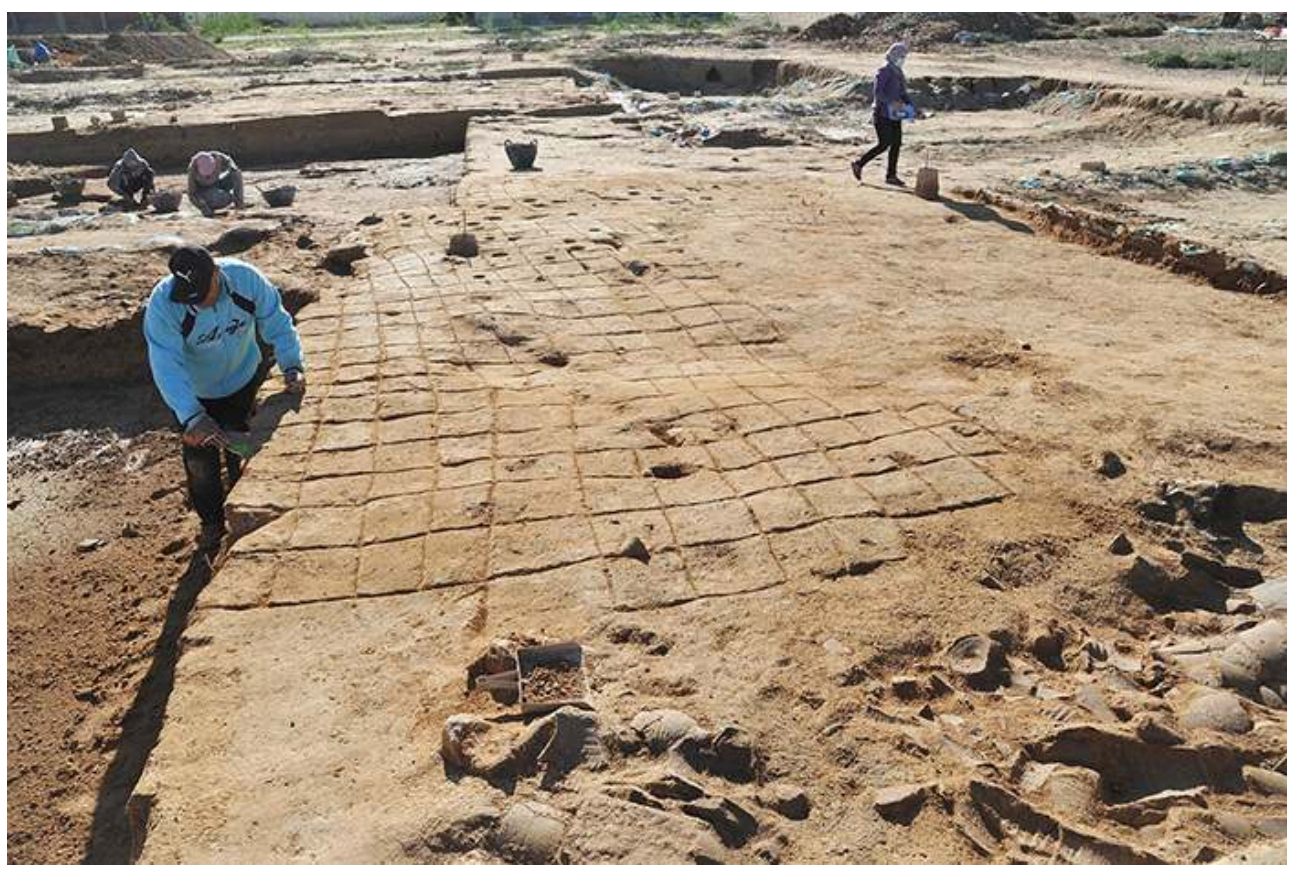

(C) Archives CEAlex. CEAlex_AKA_2020_NDMPF_007

Dans le retour sud-est, les couches de maintien du four oriental sont en contact avec le troisième niveau de sol mis au jour (US 70218) : l'US 70232 bute contre le sol, tandis que l'US 70238 passe sous ce sol. L'enlèvement de l'US 70238 a mis au jour les restes d'un quatrième niveau de sol en briques crues (US 70255) sur lequel vient buter à l'est une autre couche composée de nombreux fragments d'amphores (US 70243) (fig. 34). Ce sol est très certainement à mettre en relation avec un des sols mis au jour au sud sous l'US 70218 dans l'extension ouest du sondage 3. L'étude post-fouille permettra d'en déterminer la nature exacte. Quant à l'US 70243, elle est associée à deux autres couches d'amphores (US 70247 et 70245) : l'ensemble semble être à mettre en relation avec une surélévation et un nivellement de la zone que l'on retrouve au sud (US 70245). 
Fig. 34. Akadémia. Secteur 7 , sondage 9 , troisième sol en briques crues (US 70218) en cours de relevé, quatrième sol en brique crues (US 70255), visible au premier plan, contre lequel vient buter I'US 70243 en cours de fouille, l'ensemble est vu depuis le sud-est (V. Pichot).

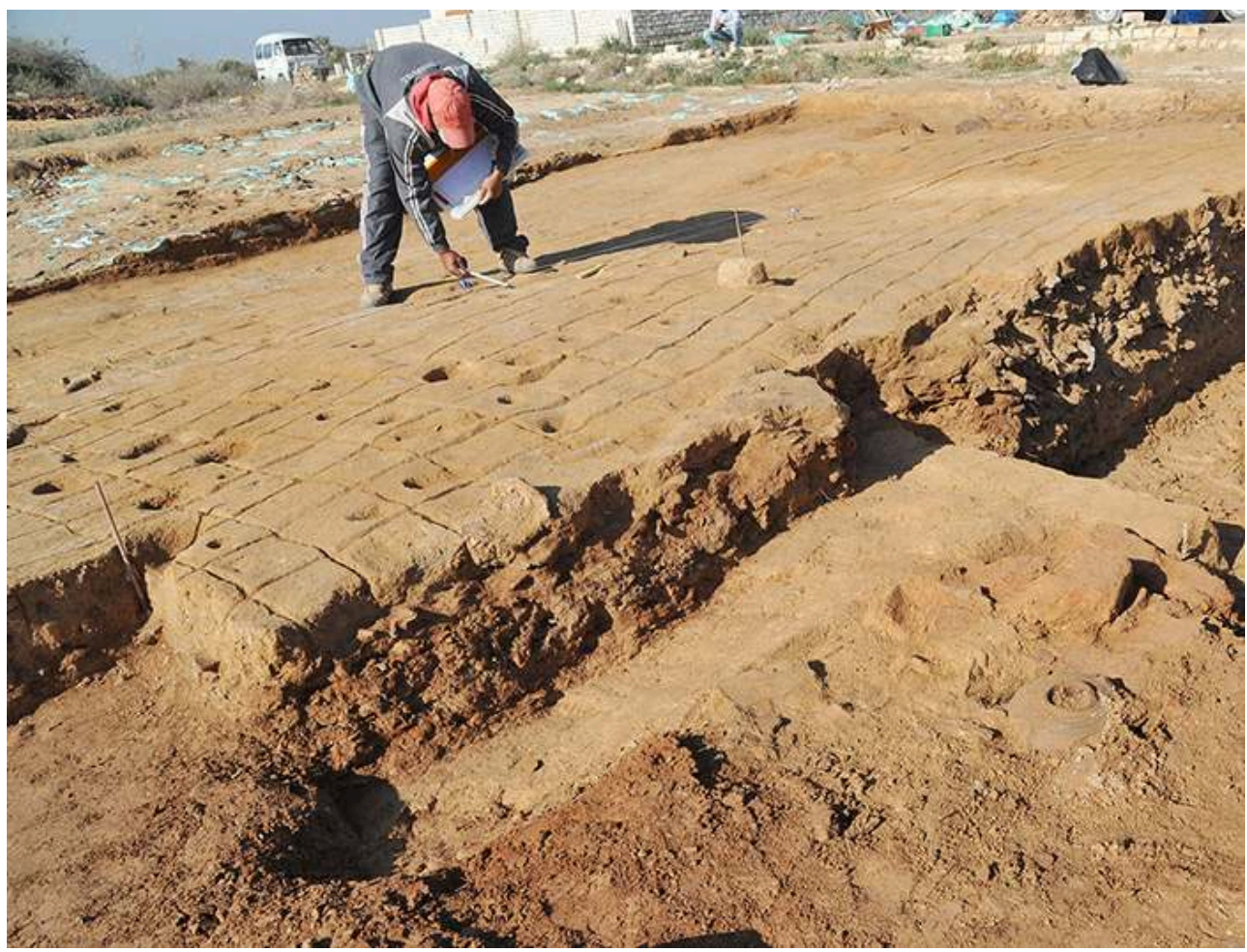

(c) Archives CEAlex. CEAlex_AKA_2020_NDMPF_008

Les niveaux inférieurs atteints dans le retour sud-est du sondage 9 sont identiques à ceux mis au jour dans la partie nord de l'extension ouest du sondage 3 (fig. 35) : un sol rouge composé de fragments de briques et d'amphores pilées mêlées à de l'argile et compactés, associé à une sorte de muret en briques cuites à l'est. La couche de détérioration de l'ensemble porte le numéro d'US 70252 (égal à 70249 au sud). 
Fig. 35. Akadémia. Secteur 7, sondage 9 partie orientale (à gauche) ; extension ouest du sondage 3 partie nord (à droite), niveaux inférieurs atteints : sol rouge compact et muret en briques cuites à l'est, vus depuis l'ouest (V. Pichot).

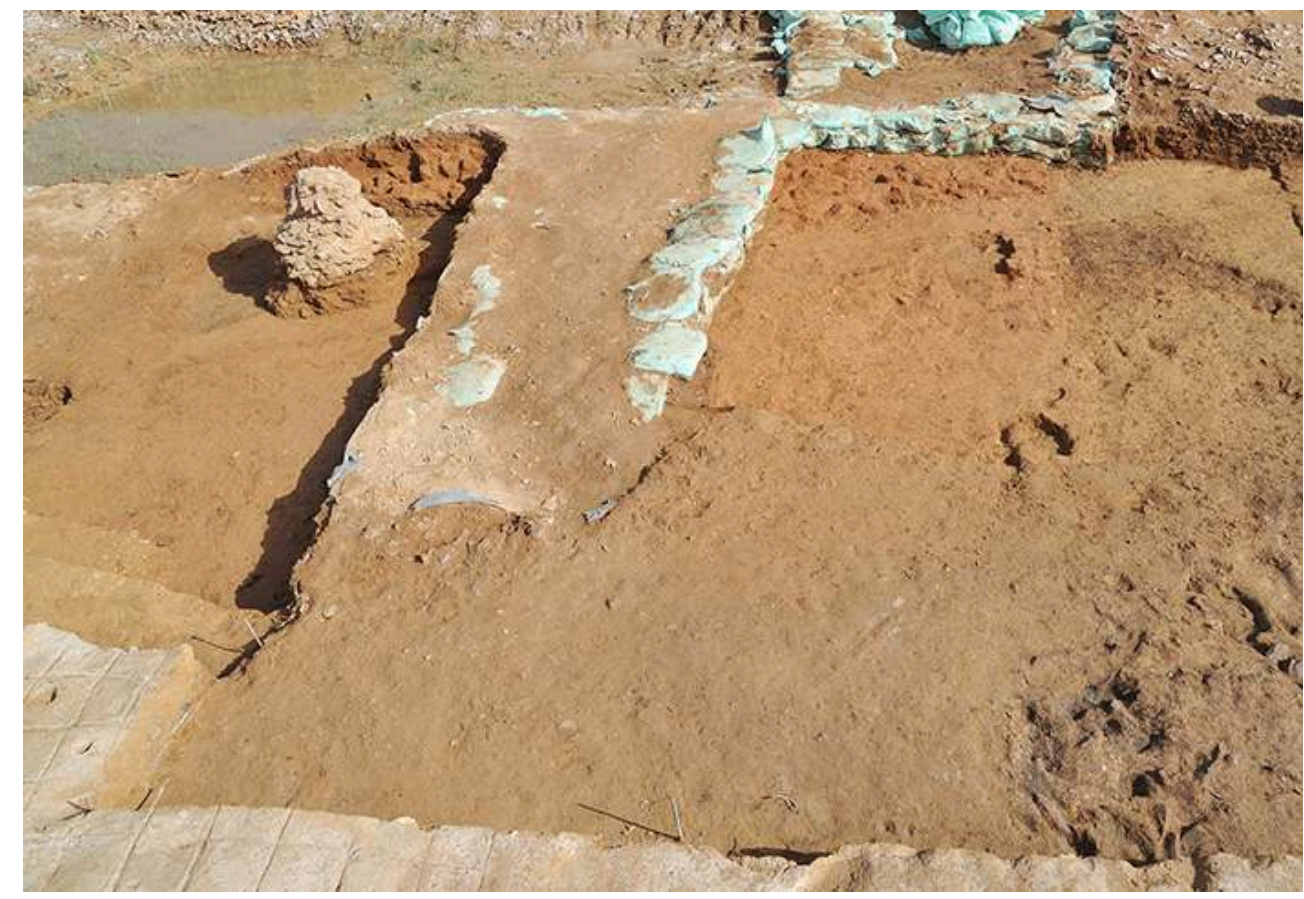

(c) Archives CEAlex. CEAlex_AKA_2020_NDMPF_009

\subsubsection{Sondage 3 et son extension ouest}

51 La réouverture du sondage 3 et de son extension ouest (comprise entre les sondages 3 et 5 et fouillée en partie en 2019) permet de faire le lien entre les sondages 7 et 8 au sud et le sondage 9 au nord (voir fig. 23). L'enlèvement du sol en briques crues découvert en 2018 et 2019 (US 70218) ainsi que d'une couche composée de fragments d'amphores (US 70220) située au nord et venant buter sur le sol a mis en évidence la présence d'un second sol en briques crues directement sous le premier.

52 Nous avons procédé au dégagement des différents sols en briques crues qui se succèdent sous le sol supérieur (US 70218/70221). Chacun de ces sols en briques crues, US 70218/70221, US 70223 (fig. 36), US 70225 (fig. 37), US 70240/70241 (fig. 38), est associé à des couches d'amphores qui se développent au nord (US 70222 en relation avec 70218/70221; US 70220 en relation avec 70223 ; US 70230, 70233, 70234 et 70235 en relation avec $70225 ; 70237$ en relation avec 70240/70241). 
Fig. 36. Akadémia. Secteur 7 , sondage 3 et son extension ouest, après enlèvement partiel du sol en briques crues 70218 , vue du sol 70223 et de la couche d'amphores associée (US 70220) depuis le nord-ouest (V. Pichot).

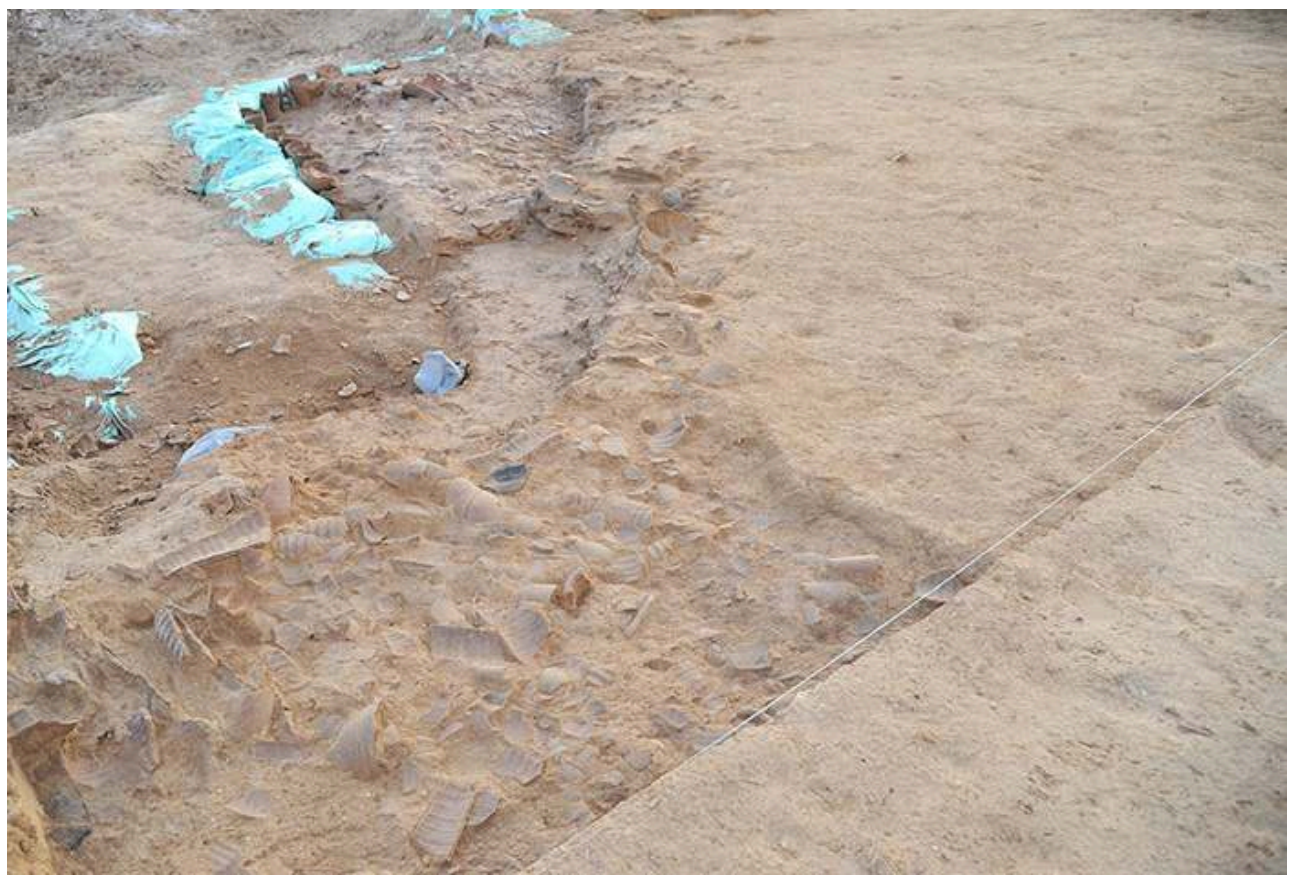

(C) Archives CEAlex. CEAlex_AKA_2020_NDMPF_010

Fig. 37. Secteur 7 , sondage 3 et son extension ouest, vue du sol 70225 depuis le nord-ouest (V. Pichot).

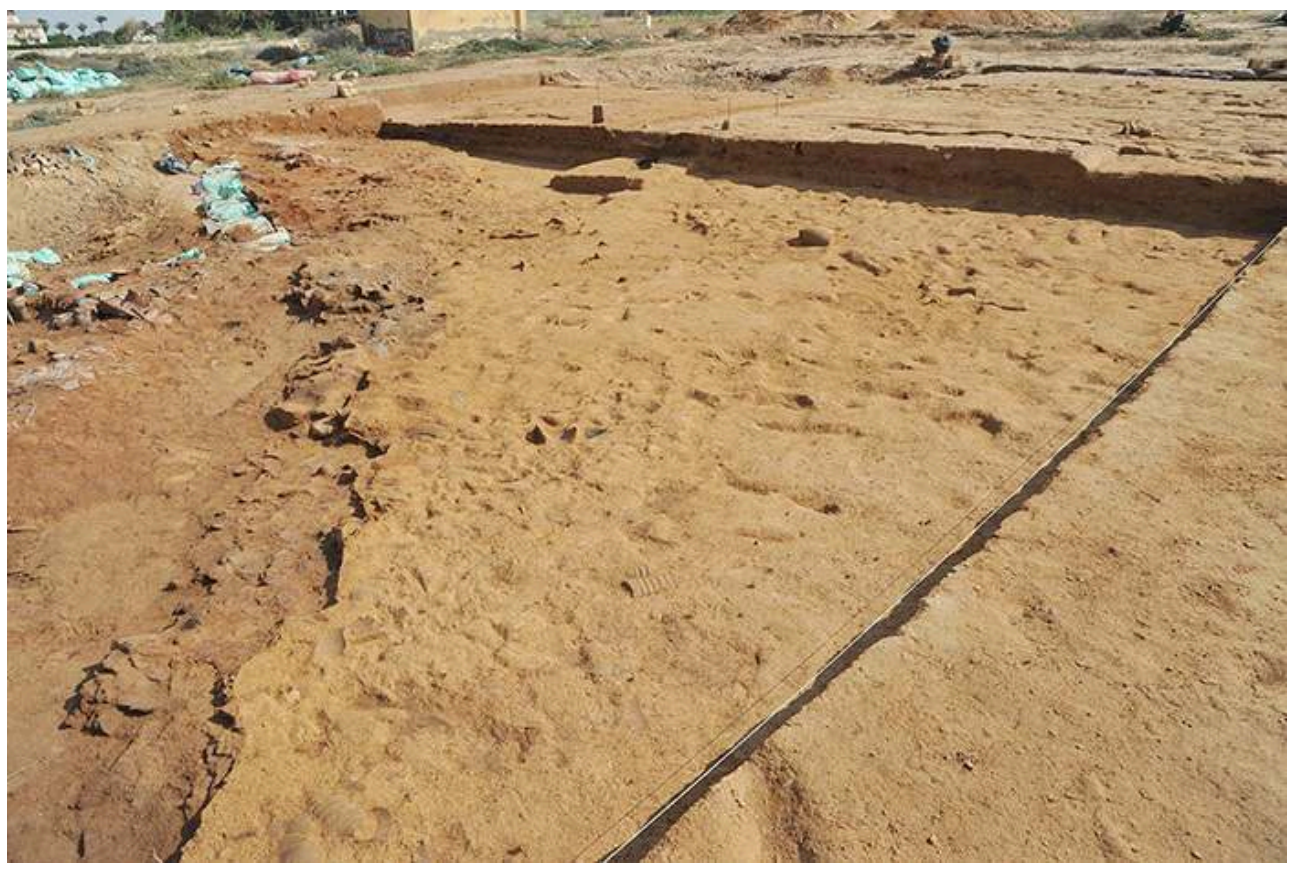

(c) Archives CEAlex. CEAlex_AKA_2020_NDMPF_011 
Fig. 38. Akadémia. Secteur 7 , sondage 3 et son extension ouest, dégagement du sol 70240/70241, vu depuis le nord-ouest (V. Pichot).

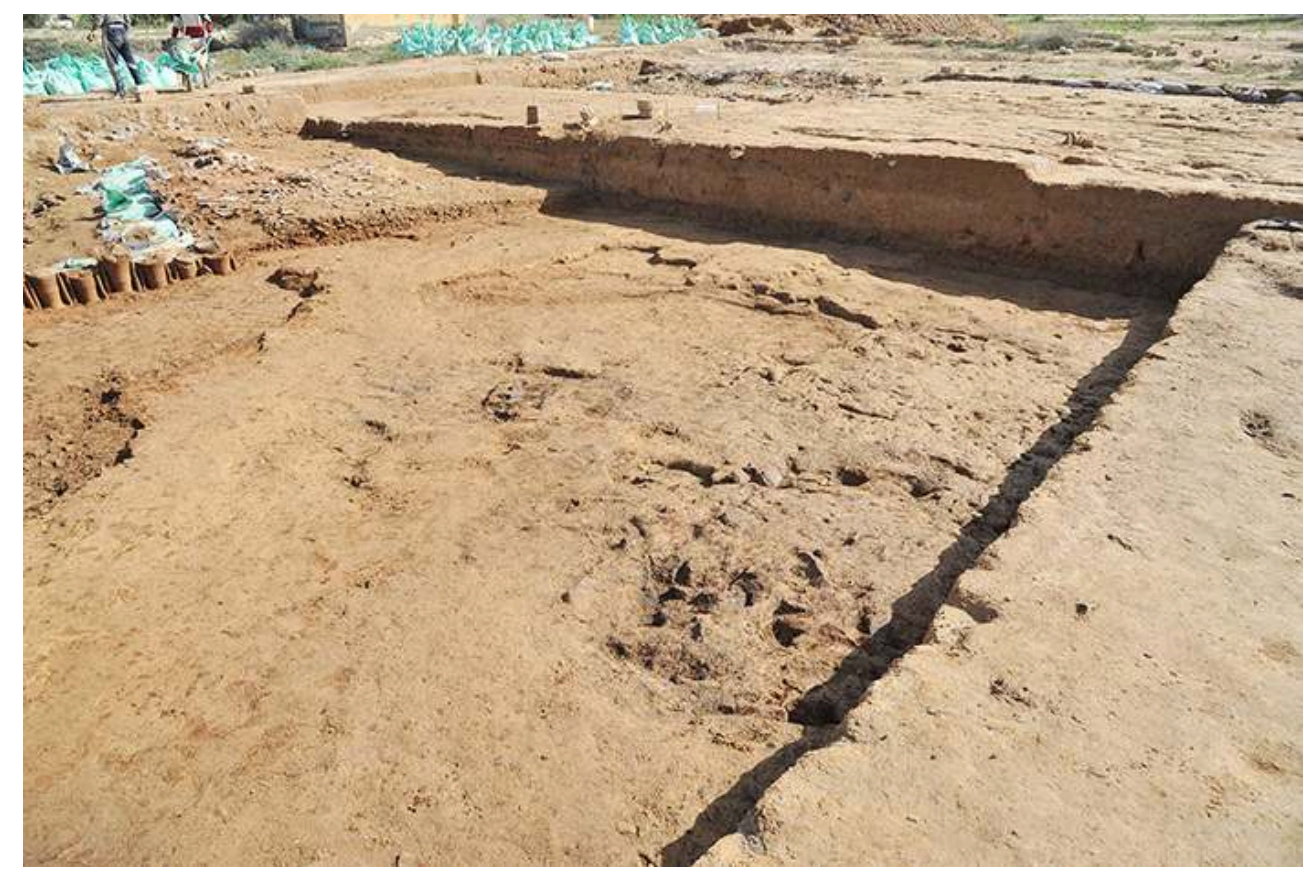

(c) Archives CEAlex. CEAlex_AKA_2020_NDMPF_012

Quelques niveaux d'occupation ont pu être repérés, caractérisés par la présence de fragments d'amphores et de céramiques, de quelques fragments de verre et de quelques éléments de faune : l'US 70222 pour le sol 70221 dans lequel a été installé un petit foyer domestique ; les US 70220, 70236, 70239, 70244 et 70246 liées à l'occupation sur le sol 70240/70241. À noter que les US 70229/70236/70239, argilo-sableuses très noires comportant de nombreux fragments millimétriques de charbon sont peut-être à mettre en relation avec un incendie. Un niveau similaire avait été repéré en 2018 dans le sondage 4 au sud.

Dans la partie nord, le niveau inférieur atteint dans ce secteur est identique à celui mis au jour dans le sondage 9: un sol rouge composé de fragments de briques et d'amphores pilées mêlées à de l'argile et compactées, associé à une sorte de muret en briques cuites à l'est (voir fig. 35 et fig. 39). La couche de détérioration de l'ensemble porte le numéro d'US 70249. C'est sur ce sol et sur ou contre le muret qu'a été installé l'alignement d'amphores retournées (US 70248) découvert lors des missions de 2018 et 2019. Les amphores ont été prélevées et leur fouille au moment de leur nettoyage a permis de valider l'hypothèse qu'elles avaient été positionnées entières lors de l'aménagement de la zone. Elles délimitaient bien deux zones distinctes d'activité : au nord, la zone de malaxage découverte lors de la mission 2016 ; au sud, une zone encore non définie caractérisée par la présence du sol rouge compacté et du muret en briques cuites localisés dans la partie nord de la zone fouillée cette année. 
Fig. 39. Akadémia. Secteur 7 , sondage 3 et son extension ouest, niveaux inférieurs atteints, vus depuis l'ouest : à droite sol rouge compacté et reste du muret en briques cuites, à gauche plateforme et fosse en cours de dégagement (V. Pichot).

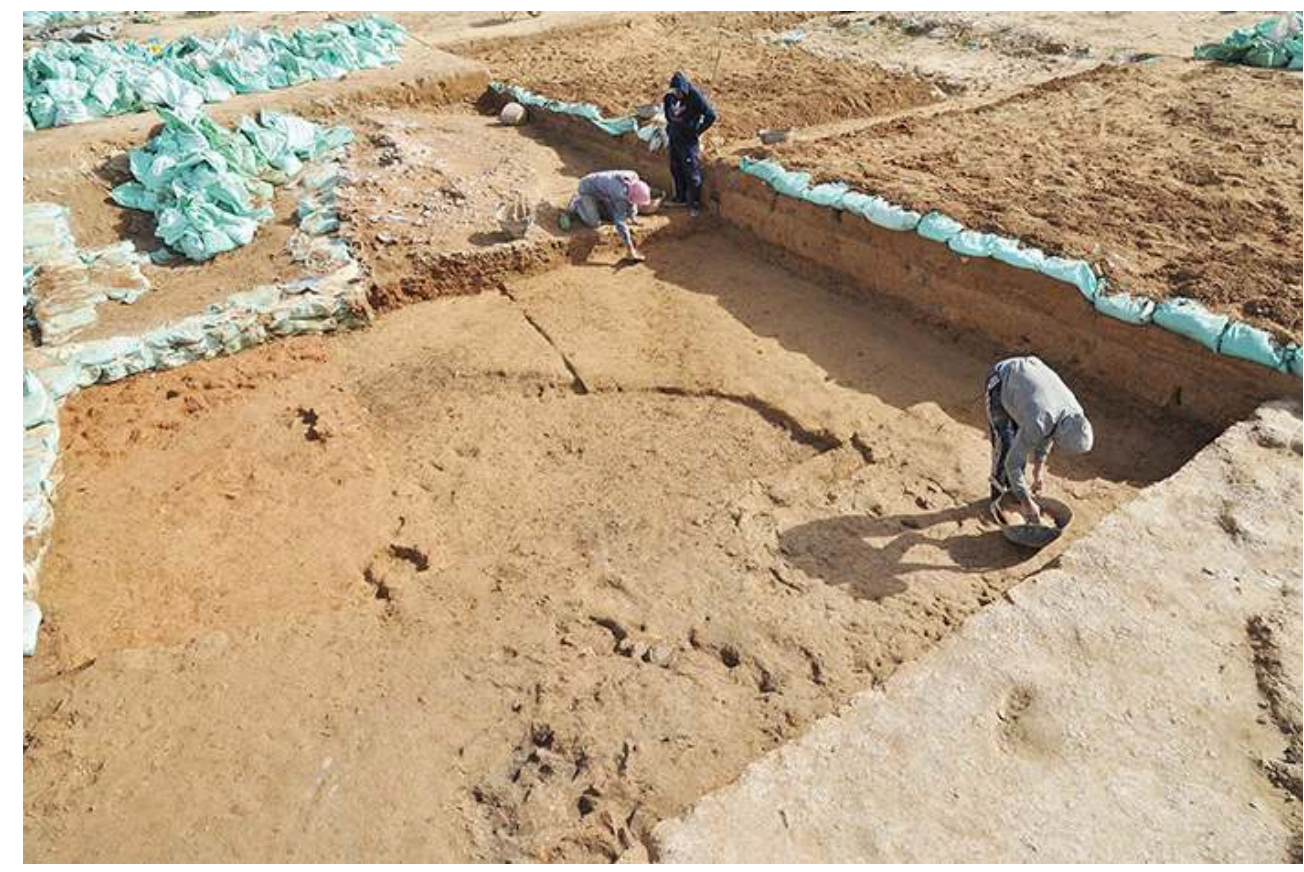

(C) Archives CEAlex. CEAlex_AKA_2020_NDMPF_013

55 Le niveau inférieur atteint dans la partie sud de ce secteur est antérieur à la mise en place du sol rouge compact et en est séparé par des couches de surélévation/ nivellement composé de sédiments argilo-sableux mêlés à des fragments d'amphores (US 70245, 70250, 70251). Il est caractérisé par une plateforme, résultat de l'aplanissement du substrat naturel dans lequel on note la présence d'une grande fosse circulaire (voir fig. 39). L'ensemble rappelle les aménagements de la zone de malaxage plus au nord, mais la présence de la nappe phréatique n'a malheureusement pas permis de confirmer la fonction de cet espace.

\subsection{Conclusion}

Les résultats des investigations menées sur l'atelier de production d'amphores d'Akadémia, un des rares exemples d'atelier fouillé en Maréotide, sont riches d'enseignement. Les recherches menées ont permis de caractériser la production de cet atelier durant le Haut-Empire et de récolter de nombreuses informations sur la chaîne opératoire ainsi que sur l'organisation du travail. Elles s'inscrivent aussi dans l'étude diachronique de la production d'amphores de la région ${ }^{18}$ développée dans le cadre du projet Maréotide par le CEAlex (voir infra § 3).

Plus largement, le site d'Akadémia participe à la connaissance de la Maréotide à l'époque romaine. Cette région fertile est exploitée de façon importante dès l'époque hellénistique et cette exploitation s'accélère encore au Haut-Empire. Le territoire était alors partagé entre différentes entités plus ou moins autonomes: les grandes villas agricoles, illustrées par la partie orientale du site d'Akadémia, géraient l'intégralité ou pour le moins une grande partie de la production. La moindre étape de la chaîne opératoire y était contrôlée : de la production agricole au transport en passant par la 
transformation des produits, la fabrication des contenants ou encore la mise en amphore. Dans le courant du III ${ }^{\mathrm{e}}$ s. apr. J.-C., la montée importante du lac ${ }^{19}$ entraîne un abandon partiel des parties basses de la rive sud du lac. Seuls les sites installés sur des promontoires rocheux continuent à être occupés de façon importante à cette époque. La majorité des activités de production artisanale se déplace à l'intérieur des terres plus au sud. Néanmoins, certains secteurs des parties basses de la rive du lac sont réservés à des activités agricoles, parfois importantes comme dans la partie occidentale d'Akadémia.

\section{Carte archéologique de la Maréotide}

Valérie Pichot, avec la collaboration d'Aude Simony et d'Ismaël Awad Responsable d'opération : Valérie Pichot (archéologue).

Participants : Aude Simony (céramologue), Ismaël Awad (topographe).

Autorité nationale présente: Le MoTA était représenté par Wahel Ahmed (Département des Antiquités d'Alexandrie) et par Ahmed Galal (Département des Antiquités sous-marines d'Alexandrie).

Le projet de carte archéologique mené par le CEAlex a pour but de récolter toutes les informations sur la Maréotide ancienne, région qui connaît actuellement une phase d'urbanisation et d'industrialisation accrue qui menace les sites archéologiques, et de mettre à la disposition du MoTA toutes ces informations sous la forme d'un Système d'information géographique $(\mathrm{SIG})^{20}$. La prospection sur le terrain est indispensable et urgente pour identifier les sites anciens et les documenter. L'autorisation de prospection 2020 accordée par le MoTA couvre les sites le long de la rive sud du lac Mariout (au nord de la route Amreya-Borg el-Arab), des îles à proximité de cette rive et les sites entre la route d'Amreya-Borg el-Arab et le chemin de fer (fig. 40).

La mission s'est déroulée du 13 juillet au 13 août 2020 à raison de 3 à 4 jours de travail sur le terrain par semaine, les jours restants étant dédiés au traitement des données de terrain (photographies, description des sites, mise au propre des informations récoltées, etc.). En raison de la pandémie de Covid-19, cette mission s'est déroulée en été, saison qui n'est pas la plus appropriée pour effectuer des prospections en Maréotide. La chaleur qui assèche le sol, la réverbération importante sur le sédiment clair ainsi que la végétation basse plus importante en été diminuent la lisibilité de la surface et la possibilité de repérer d'éventuelles structures en surface. C'est pour ces raisons que nous avons choisi de ne pas prospecter de secteurs inconnus et que la mission s'est focalisée sur des sites déjà prospectés dont la documentation, notamment céramologique, devait être complétée.

Le choix s'est porté sur 11 sites qui avaient fait l'objet de prospection en 2019, excepté le site GMR0116 prospecté en 2016, le site GMR0133 en 2017 et le site GMR0158 en 2018. En outre, une rapide visite a été effectuée sur le site de Sidi Mahmoud (GMR0016) pour vérifier l'existence d'un karm répéré au sud du site sur les anciennes cartes (voir tableau 1 et fig. 41). 
Fig. 40. Carte archéologique de la Maréotide. Délimitation de la zone de prospection et sites prospectés en 2020 (Google Earth, V. Pichot).

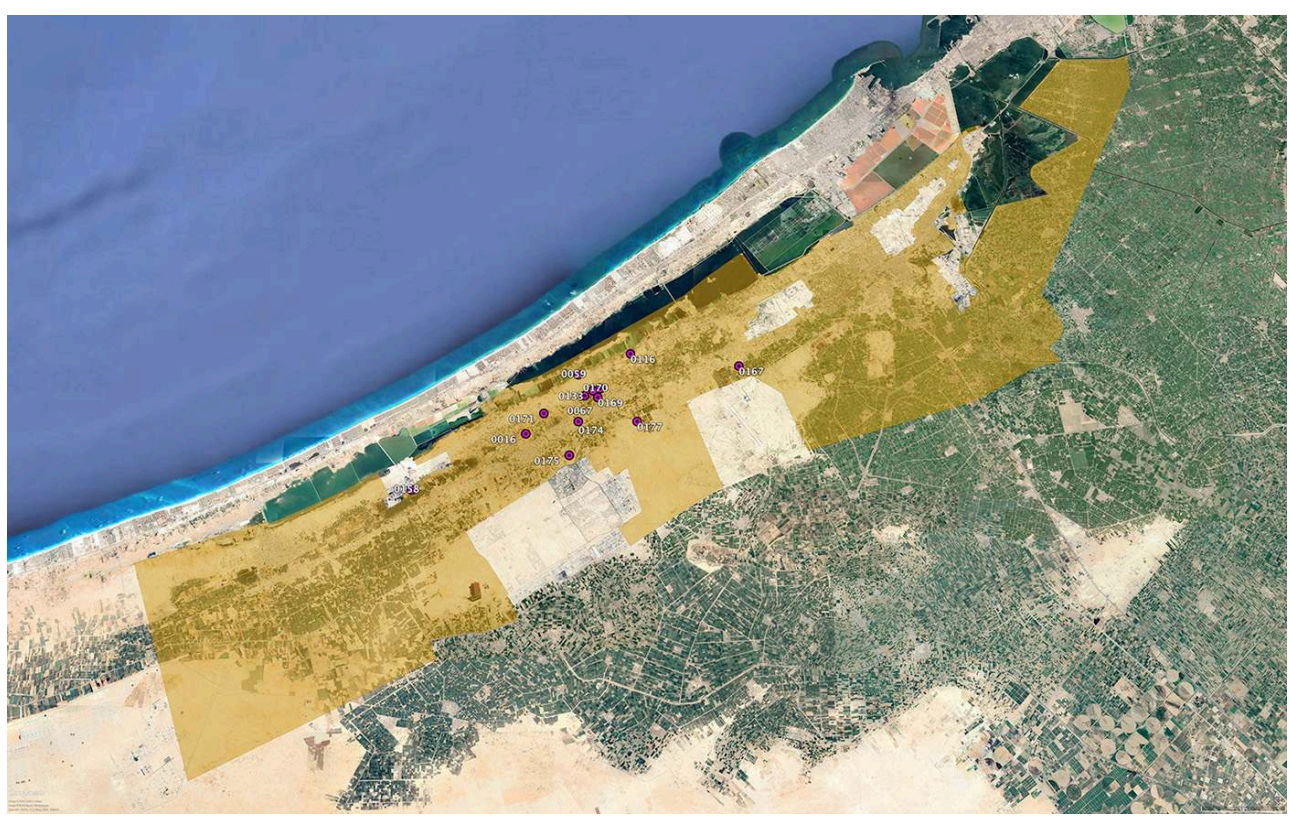

(c) Archives CEAlex. CEAlex_GMR_2020_NDMCN_001

Tableau 1. Liste des sites prospectés en juillet - août 2020.

\begin{tabular}{|c|c|c|c|c|c|c|c|c|c|c|c|}
\hline No. GEOMAR & Nom & fite & 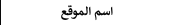 & Région & المنطقة & Latitude (North) & Longitude (East) | & Surface $\left(\mathrm{m}^{2}\right)$ & Fonction & \multicolumn{2}{|c|}{ Chronologie } \\
\hline - & डावाली & fhoud & 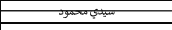 & Aorgthatab & (1) & $30.990820^{\circ}$ & $29.548400^{\circ}$ & 18536 & Habtissementectretitien/Karm! & tooque romaine tardive & \\
\hline GMR0059 & sidi solim & el-Tayer & سيدي سليمان الطاير & Nagaa E-Sanagra & نجيع سشاقرة & $30.946333^{\circ}$ & $29.582967^{\circ}$ & 510055 & Village et karms & 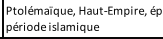 & \\
\hline GMR0116 & Qasiminy & |arrière & القاسبية محير & Qasimiva & |لاساسية & $30.959036^{\circ}$ & $29.618377^{\circ}$ & 19786 & Carrière/Nécropole & $\begin{array}{l}\text { aique } 2 \text { zone devassage } \\
\text { omaine tardive }\end{array}$ & \\
\hline Gмво133 & kRO & 貄 & كرئرقم 333 & Bahig & 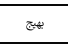 & $30.936620^{\circ} \mathrm{N}$ & $29.593063^{\circ} \mathrm{E}$ & 23842 & Karm et bấtiments agricoles & $\begin{array}{l}\begin{array}{l}\text { Helléristique, Haut-Empire et } \\
\text { tardive }\end{array} \\
\text { to }\end{array}$ & ine \\
\hline GMR0158 & Karm & hatf & 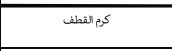 & larba & ليابياتيات & $30.878278^{\circ}$ & $29.473912^{\circ}$ & 237258 & $\begin{array}{l}\text { Village ou trés grande villa/ } \\
\text { Karm/Atelier d'amphores }\end{array}$ & 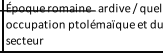 & $\begin{array}{l}\text { esd'une } \\
\text { estedans le }\end{array}$ \\
\hline GMR0167 & кRо & & كركر قيم 83 & wareya & الهوارية & b.951431 & $29.692239^{\circ}$ & 14167 & Karm & Iique, Haut- & naine \\
\hline GM & кROF & & 32 & Bahi & به & & & & $\mathrm{Kat}$ & & \\
\hline GMR01 & & & & Bahieg & . & & $0^{\circ}$ & & & Haut-E & \\
\hline GMR0171 & $\begin{array}{c}\text { Sidi Hamiq } \\
\text { soliman }\end{array}$ & $\begin{array}{l}\text { Saballah } \\
\text { Shamatata }\end{array}$ & سياي حمبد جاب الثله سليمان شم. & Nagaa El-Sanaqra & نجيع لسناقرة & 30.922983" & $29.560151^{\circ}$ & 34329 & Karm & Haut-Empire et époque romain & ine tardive \\
\hline GMR0174 & cheminde & ter $\mathrm{km} 46$ & كـ 46 سكة حديد & Nagaa Ahmed & نجيعأمد & $30.918091^{\circ}$ & $29.583408^{\circ}$ & 164594 & Carrière et village & 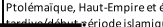 & que \\
\hline GMR0175 & Sidi & ael Shayet & سبيدي عبالهاله الشايط & Borg El-Arab & 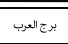 & 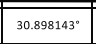 & $29.577736^{\circ}$ & 30812 & Karm & $\begin{array}{l}\text { Haut-Emp } \\
\text { periodeis }\end{array}$ & \\
\hline GMR0177 & Shaner $\mathrm{k}$ & lowhoh & شاهر & Borg El-Arab & برجاج العرب & $30.918030^{\circ}$ & $29.622914^{\circ}$ & 211926 & grandevi & 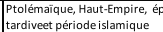 & poque romaine \\
\hline
\end{tabular}


Fig. 41. Carte archéologique de la Maréotide. Zoom sur la zone de la prospection 2020 et sites prospectés (Google Earth, V. Pichot).

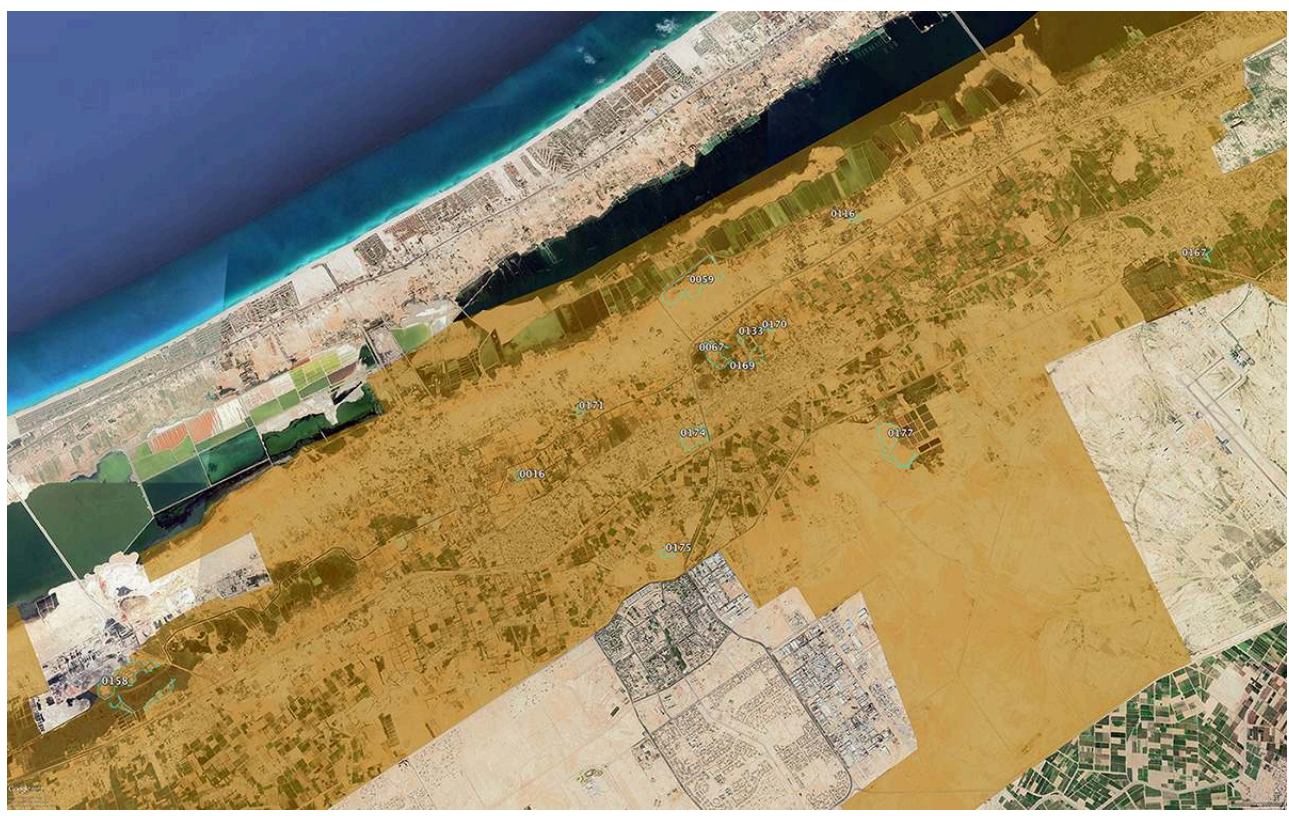

(c) Archives CEAlex. CEAlex_GMR_2020_NDMCN_002

\subsection{Présentation de sites prospectés : le secteur d'Huwareya et de} Qasimiya

3.1.1. GMR0167 - KR0083

(fig. 42)

Région de Huwareya 
Fig. 42. Carte archéologique de la Maréotide. Croquis du site GMR0167 - KR0083 : en bleu, limites actuelles du site ; en vert, anomalie (karm) repérée sur Landsat 1984 ; en orange, anomalies (karm) topographié en 1914 ; en jaune, anomalies (karm) topographiées en 1920 ; en rouge, anomalies (karm) topographiées en 1940 (V. Pichot).

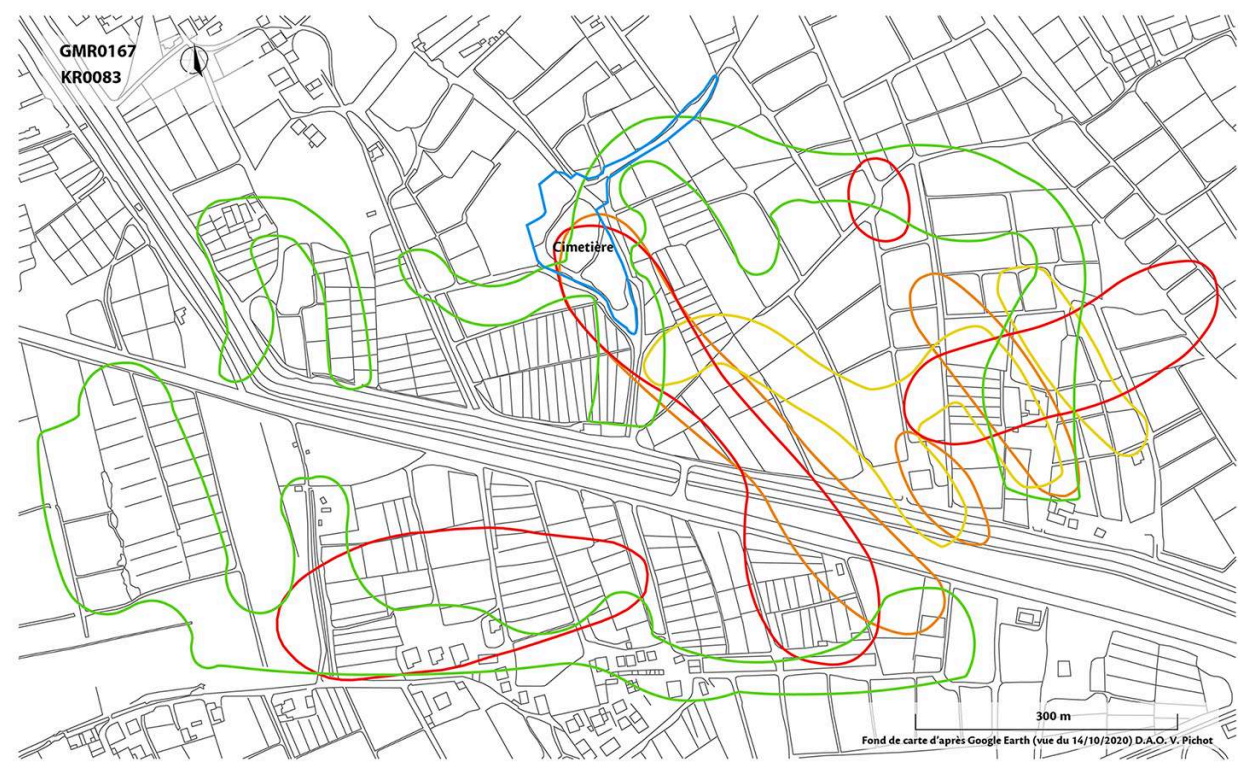

(C) Archives CEAlex. CEAlex_GMR_2020_NDMCN_003

61 Située à $4,3 \mathrm{~km}$ au sud-est du site d'Akadémia, entre la ride IV et la ride $\mathrm{V}^{21}$, une anomalie triangulaire repérée sur les images satellites Google Earth correspond aux vestiges d'un grand karm complexe, composé de trois karms, topographié en partie sur les cartes anciennes (survey de 1914, 1920 et 1940) et visible sur l'image satellite Landsat de 1984. Cette anomalie correspond à l'angle nord-est de l'enceinte du karm central et à une portion de la limite ouest de l'enceinte du karm oriental.

L'anomalie triangulaire repérée sur Google Earth se présente sur le terrain sous la forme d'une proéminence mesurant $212 \mathrm{~m}$ en nord-ouest-sud-est composée d'un sédiment argileux jaune-orange, vestige du talus de l'enceinte. Cette portion de talus a été préservée grâce à la présence d'un cimetière moderne dans sa partie centrale. Le reste du talus a été rasé à l'est pour l'implantation d'une piste, tandis que les parties nord et sud ont été décaissées pour la mise en culture du secteur. De nombreux blocs sont épars sur l'ensemble du site.

63 La partie de l'anomalie au sud du cimetière livre en surface de nombreux blocs épars (fig. 43) indiquant la présence de bâtiment(s) mais sans lisibilité en surface, ainsi que de nombreux fragments de céramiques. L'extrémité sud du triangle avait fait l'objet en 2018-2019 d'un début de destruction qui avait fait apparaître la présence de structures enfouies. L'ensemble a été légèrement remblayé depuis, masquant les murs avec enduit qui apparaissaient en 2019. On note dans ce secteur de nombreux blocs épars, des fragments de céramique, d'enduit et de mortier à fonction hydraulique (fig. 44). 
Fig. 43. Carte archéologique de la Maréotide. GMR0167, partie occidentale de la zone située au sud du cimetière, vue depuis le sud (A. Simony).

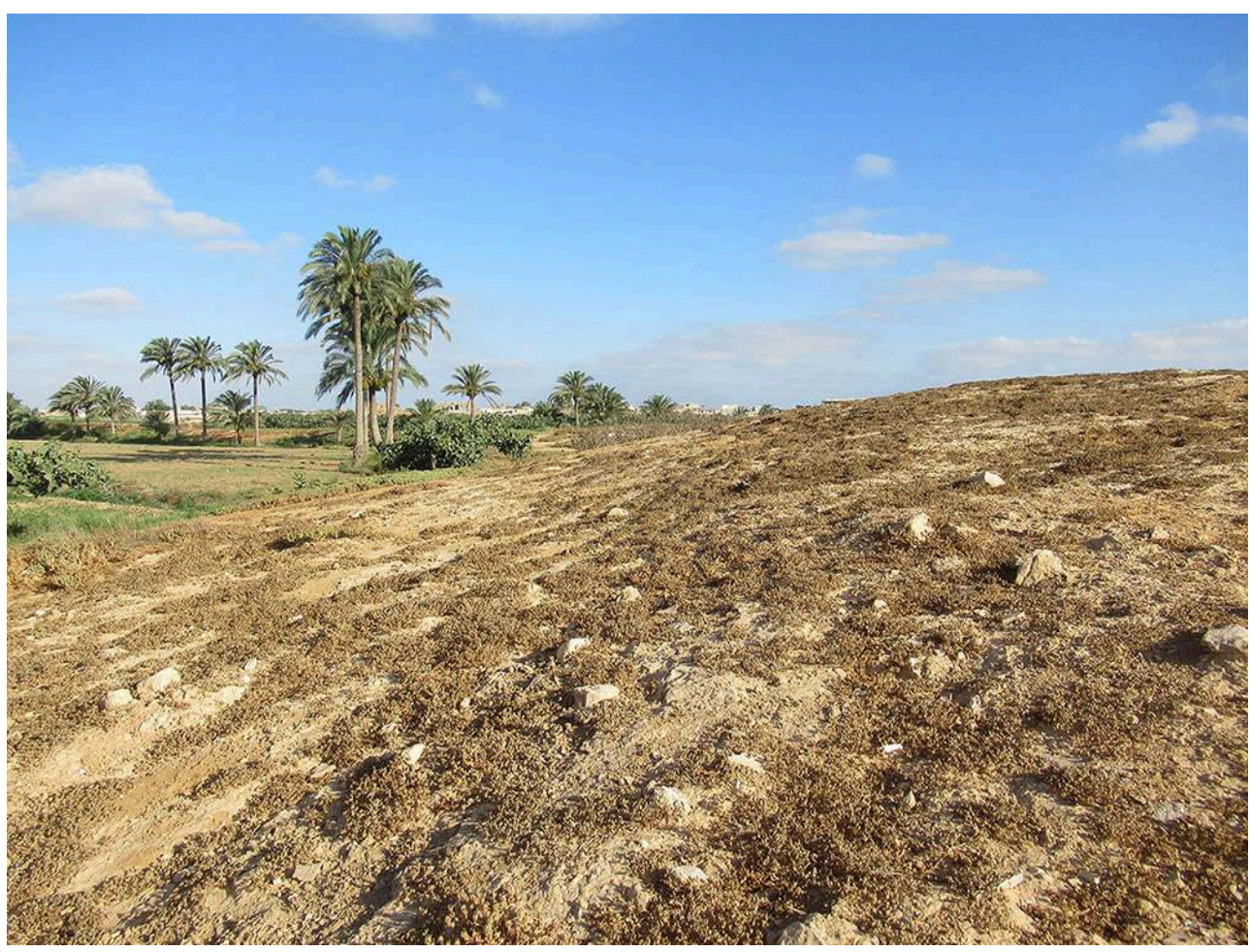

(c) Archives CEAlex. CEAlex_GMR_2020_NDMPF_001

Fig. 44. Carte archéologique de la Maréotide. GMR0167, extrémité sud du talus, vue depuis le sud (V. Pichot).

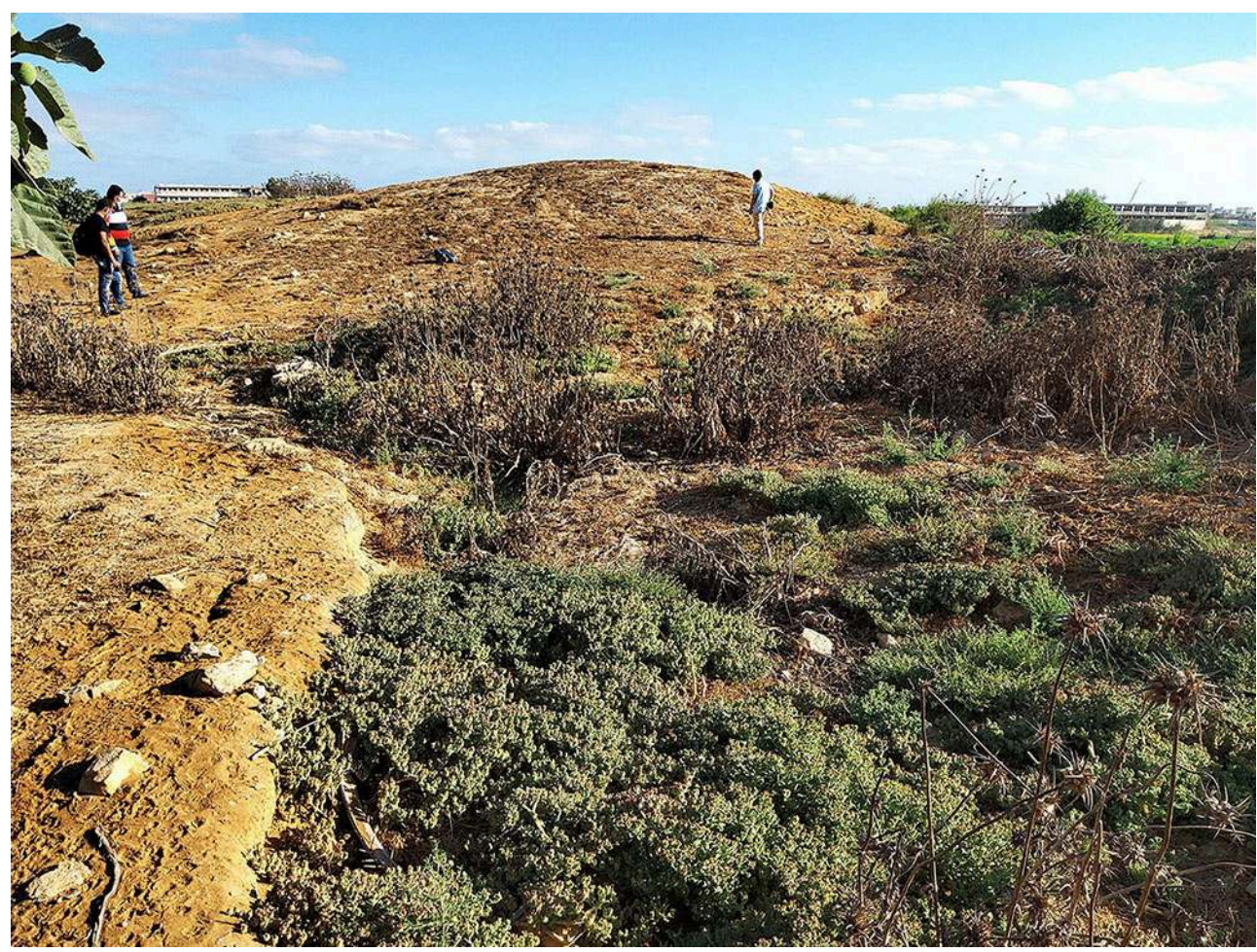

(C) Archives CEAlex. CEAlex_GMR_2020_NDMPF_002 
Dans la partie nord-ouest de l'anomalie, un secteur décaissé (fig. 45) donne accès à une partie de la stratigraphie du site (intérieur du karm): murs, sols, niveaux de drainage (?) et niveaux d'occupation apparaissent sur plus de $2,5 \mathrm{~m}$ de hauteur; l'ensemble est installé directement sur une couche argileuse orange qui semble être le substrat naturel. Au nord-est de ce grand effondrement l'anomalie triangulaire a été décaissée pour une mise en culture non aboutie : la zone a été comblée par des remblais provenant du décaissement d'une autre partie du site, ce qui empêche la lecture des stratigraphies; une partie de l'anomalie a été conservée sous la forme d'une languette orientée nord/sud et qui livre les vestiges de structures en pierres (murs?).

Fig. 45. Carte archéologique de la Maréotide. GMR0167, secteur décaissé au nord-ouest du site, vu depuis le nord-est (A. Simony).

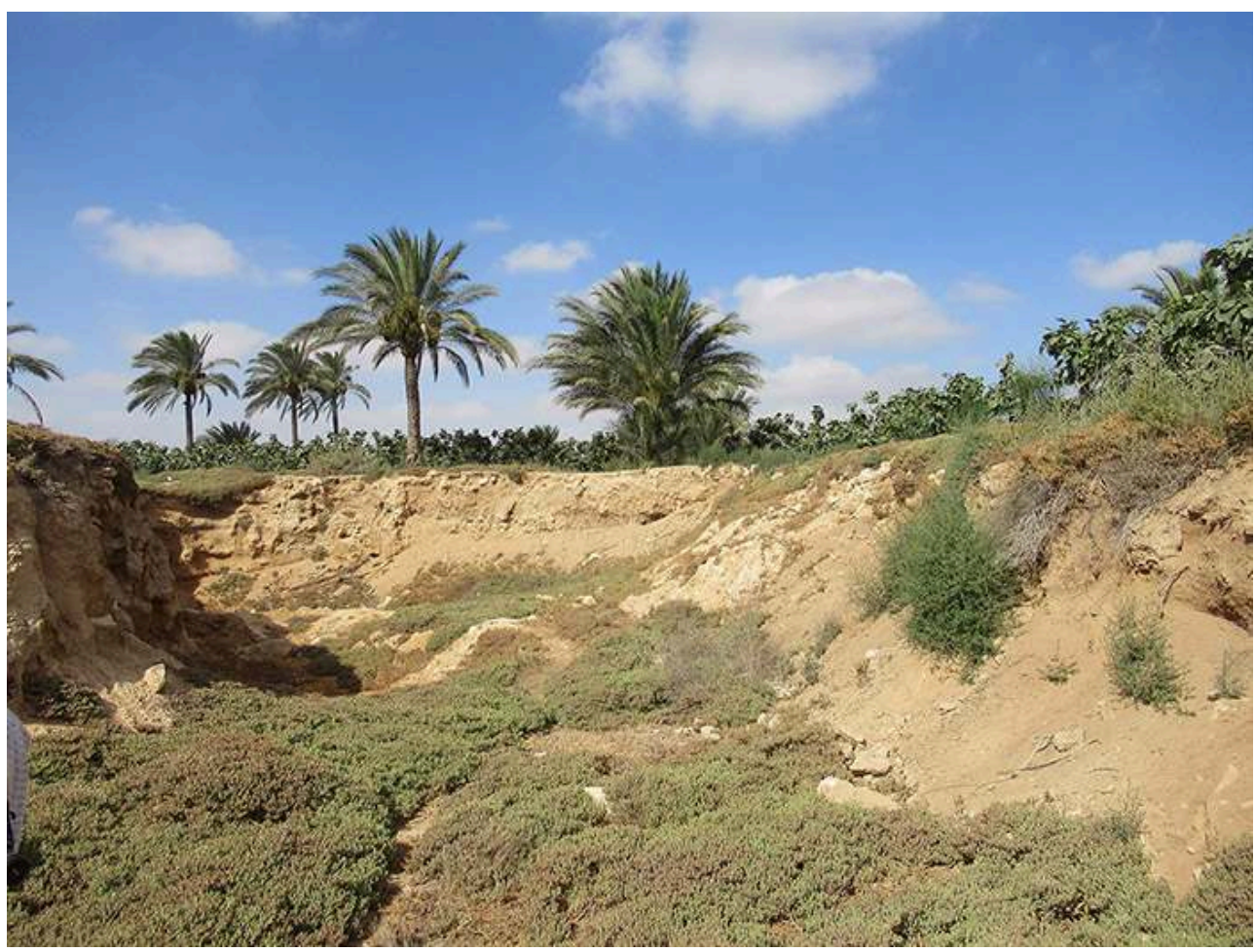

(c) Archives CEAlex. CEAlex_GMR_2020_NDMPF_003

Partant du nord-est de l'anomalie triangulaire, un talus composé de sédiment argileux jaune-orangé se développe vers le nord-est sur plus de $200 \mathrm{~m}$. Il s'agit de la limite nord du karm oriental. Le talus, arasé en partie et nivelé, a perdu une grande partie de son épaisseur par le creusement du secteur pour la mise en culture. Dans sa partie orientale une proéminence présente de nombreux blocs épars en surface ou mêlés au sédiment argileux (fig. 46), qui indiquent les vestiges d'un bâtiment enfoui dans ce secteur de l'enceinte. 
Fig. 46. Carte archéologique de la Maréotide. GMR0167, vestige du talus nord du karm, partie orientale de la proéminence conservée, vue depuis le sud-ouest (A. Simony).

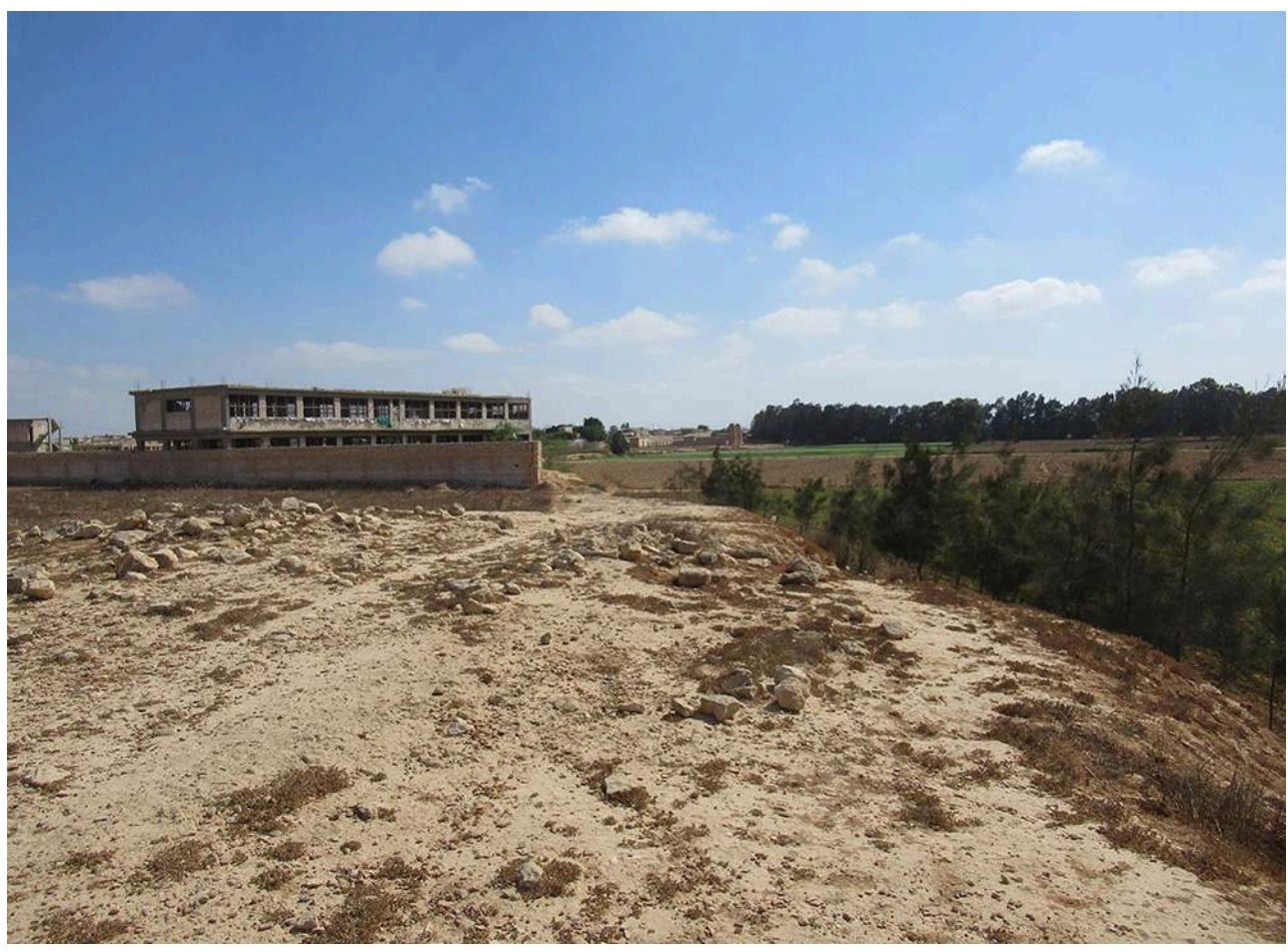

(C) Archives CEAlex. CEAlex_GMR_2020_NDMPF_004

Le mobilier en surface, assez abondant, date l'occupation du Haut-Empire (AE 3, AE 4, marmite, Eastern Sigillata $B$ ) et de l'époque romaine tardive/début de la période islamique (LRA 1, LRA 5/6, marmite à bord biseauté, $L R \mathrm{D}$ ). Une anse d'amphore cnidienne (atelier de Muhaltepe, monogramme) datée de la fin du IV ${ }^{\mathrm{e}}$-début III ${ }^{\mathrm{e}}$ s. av. J.C., trouvée en 2019, ainsi que des fragments d'amphores rhodiennes, d'amphores de Cos et d'AE 2 datées de la période ptolémaïque retrouvés dans la partie sud du site, indiquent une occupation très ancienne du site.

\subsubsection{GMR0116 - Qasimiya carrière}

\section{Région de Qasimiya}

À plus de $500 \mathrm{~m}$ à l'ouest du GMR0066 (Qasimiya nécropole), qui comporte également des tombes et des carrières, la grande carrière antique quasi quadrangulaire de GMR0116 est située sur le sommet de la ride. Des structures (puits d'accès, murs) ont été mises en évidence lors des prospections de 2016 à proximité immédiate ainsi qu'un bloc extrait et abandonné au nord de la carrière. Des escaliers sont taillés dans la paroi nord. Une grande salle rectangulaire creusée dans la paroi sud appartenait peut-être à une tombe souterraine et a servi probablement d'étable au cours du $\mathrm{xx}^{\mathrm{e}} \mathrm{s}$. fig. 47). Le mobilier est fragmenté, peu abondant, mais les tessons sont relativement bien conservés. La fourchette chronologique est large, allant de l'époque hellénistique à l'époque romaine tardive. Ce matériel indiquerait, outre l'activité de carrière et l'occupation liée à la tombe souterraine très certainement hellénistique, une zone de passage sur la ride largement fréquentée. 
Fig. 47. Carte archéologique de la Maréotide. GMR0116, grande carrière, grande salle souterraine creusée dans la paroi sud et transformée en étable au cours du $x^{e} \mathrm{~s}$., vue depuis le nord-ouest (V. Pichot).

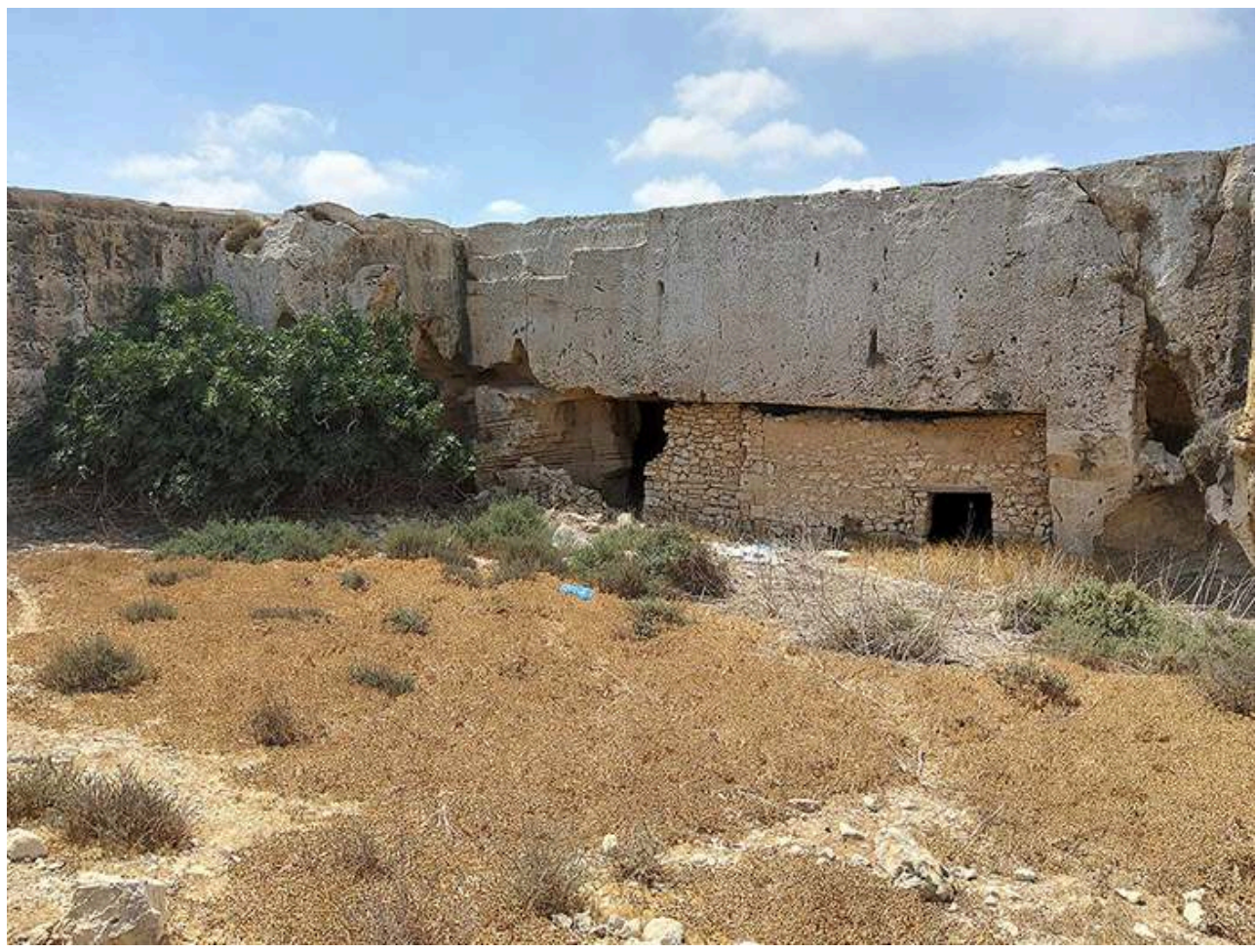

(c) Archives CEAlex. CEAlex_GMR_2020_NDMPF_005

68 À $150 \mathrm{~m}$ à l'est de cette carrière deux anomalies ont été repérées sur les images satellites Google Earth. La prospection de 2020 avait pour but de documenter ces anomalies situées à une dizaine de mètres l'une de l'autre. Il s'agit de deux tombes creusées dans le rocher (fig. 48-51), composées chacune d'un large puits associé à un vestibule auquel on accède par un escalier et une rampe (?) située à l'est. Les parois des puits montrent la présence de loculi. Tous les loculi étaient ouverts et semblent avoir été vidés de leur contenu (ce point pourrait être vérifié en nous munissant de l'équipement adéquat pour descendre dans les puits). Le mobilier repéré aux abords de ces deux tombes est peu abondant et très fragmenté (il s'agit en effet principalement de fragments de panses). Quelques éléments ont néanmoins pu être identifiés tel un fragment d'amphore de Cos et un fragment d'AE 2 datés de l'époque ptolémaïque ainsi qu'une amphore $L R A$ 1, datée de la période romaine tardive. 
Fig. 48. Carte archéologique de la Maréotide. GMR0116, tombe nord creusée dans le rocher, large puits situé à l'ouest, vu depuis le sud-est (V. Pichot).

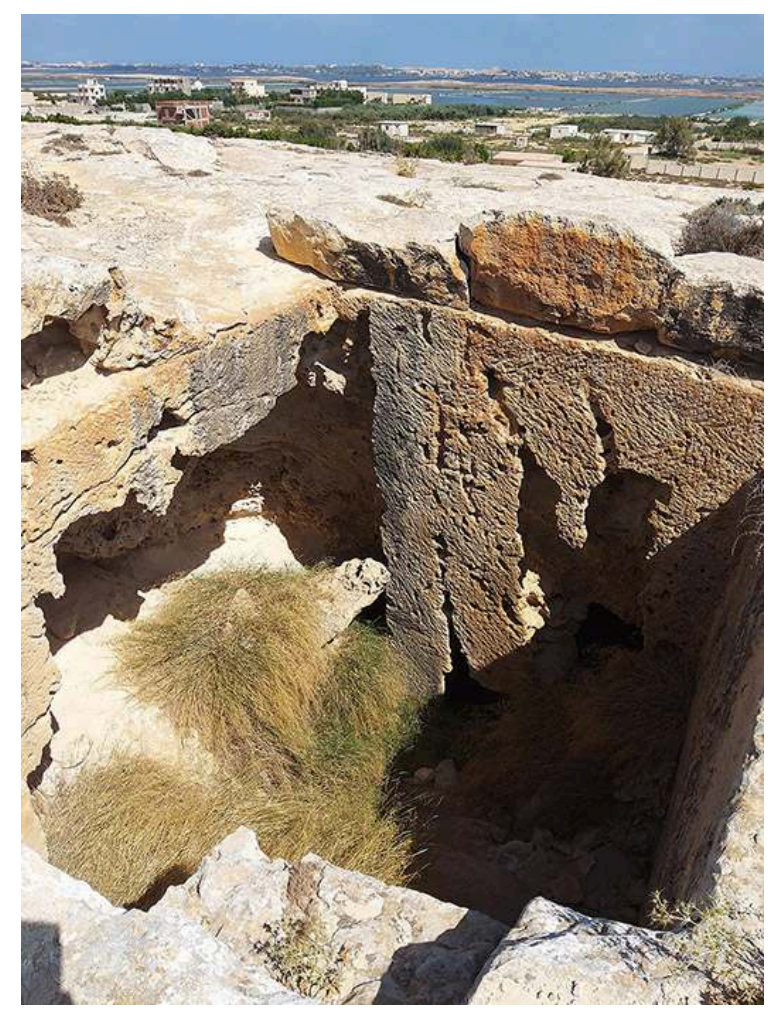

(C) Archives CEAlex. CEAlex_GMR_2020_NDMPF_006 
Fig. 49. Carte archéologique de la Maréotide. GMR0116, tombe nord creusée dans le rocher, accès au vestibule vu depuis l'est (V. Pichot).

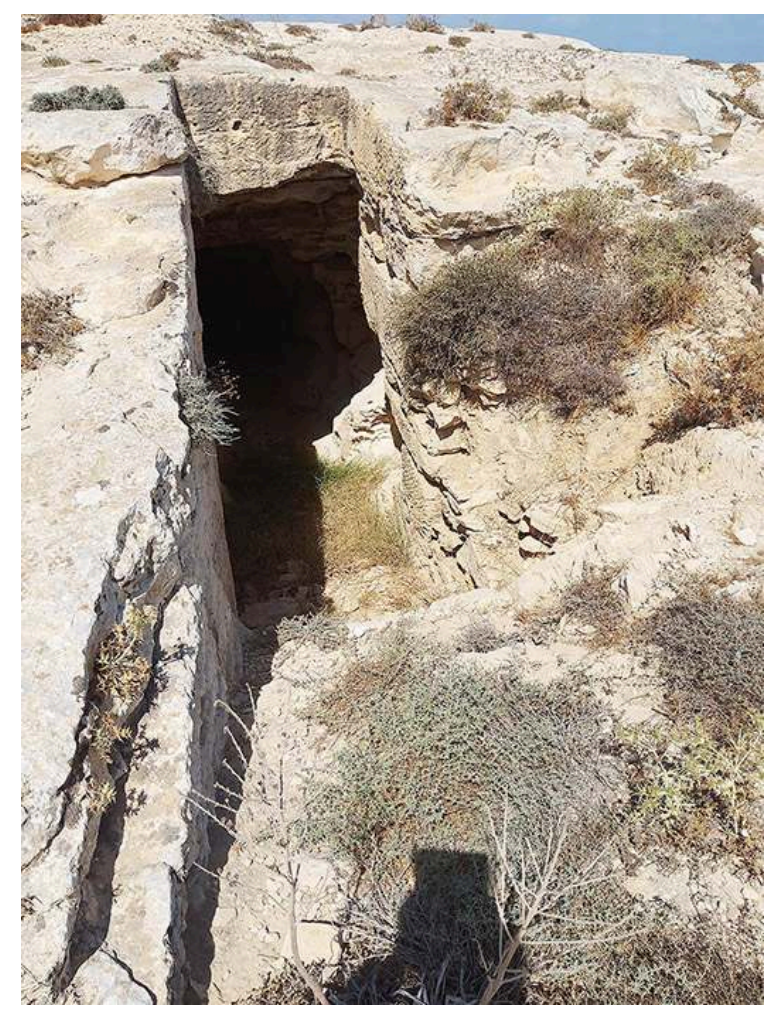

(C) Archives CEAlex. CEAlex_GMR_2020_NDMPF_007

Fig. 50. Carte archéologique de la Maréotide. GMR0116, tombe sud creusée dans le rocher, large puits situé à l'ouest, vu depuis le sud (V. Pichot).

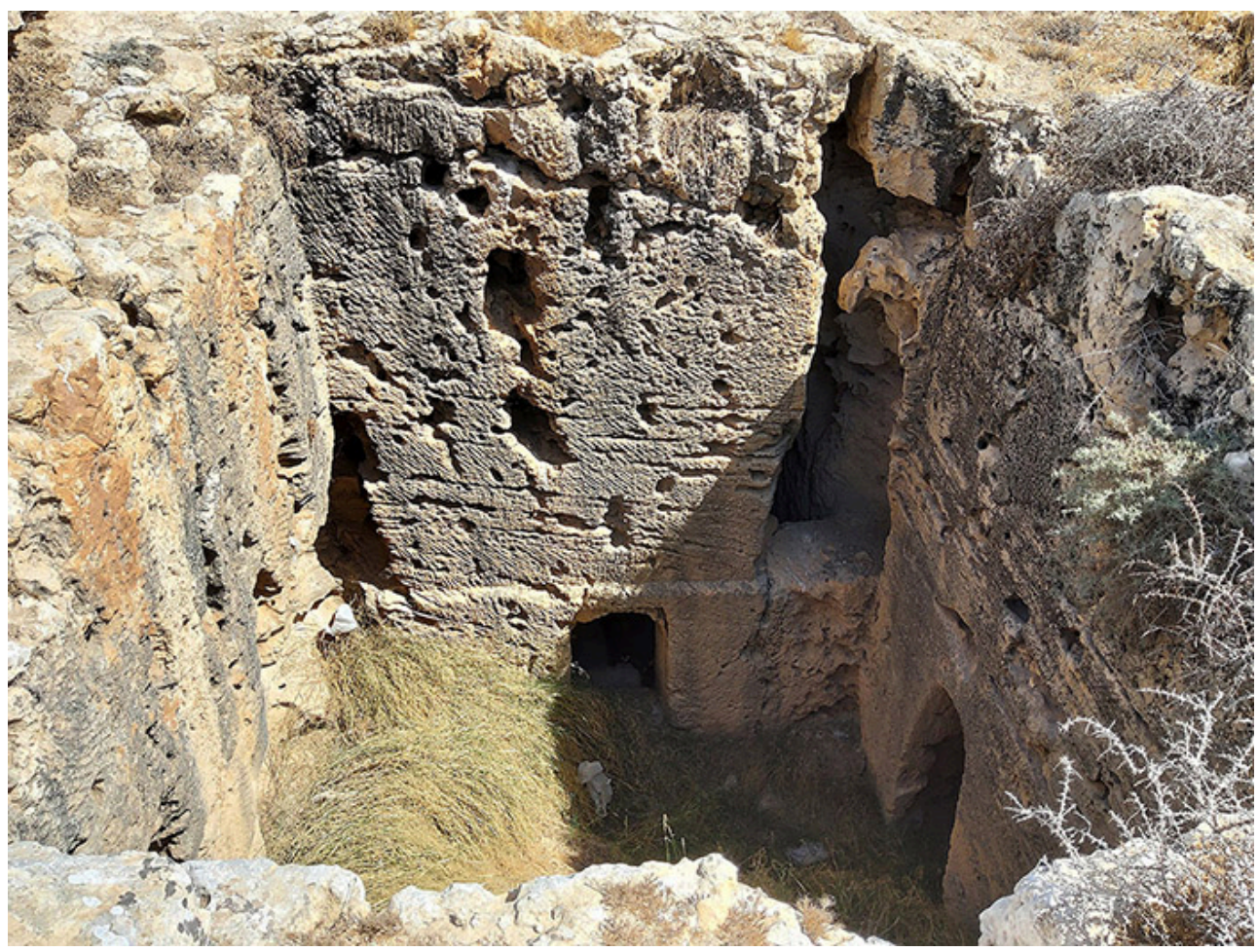

(c) Archives CEAlex. CEAlex_GMR_2020_NDMPF_008 
Fig. 51. Carte archéologique de la Maréotide. GMR0116, tombe sud creusée dans le rocher, accès au vestibule vu depuis l'est (V. Pichot).

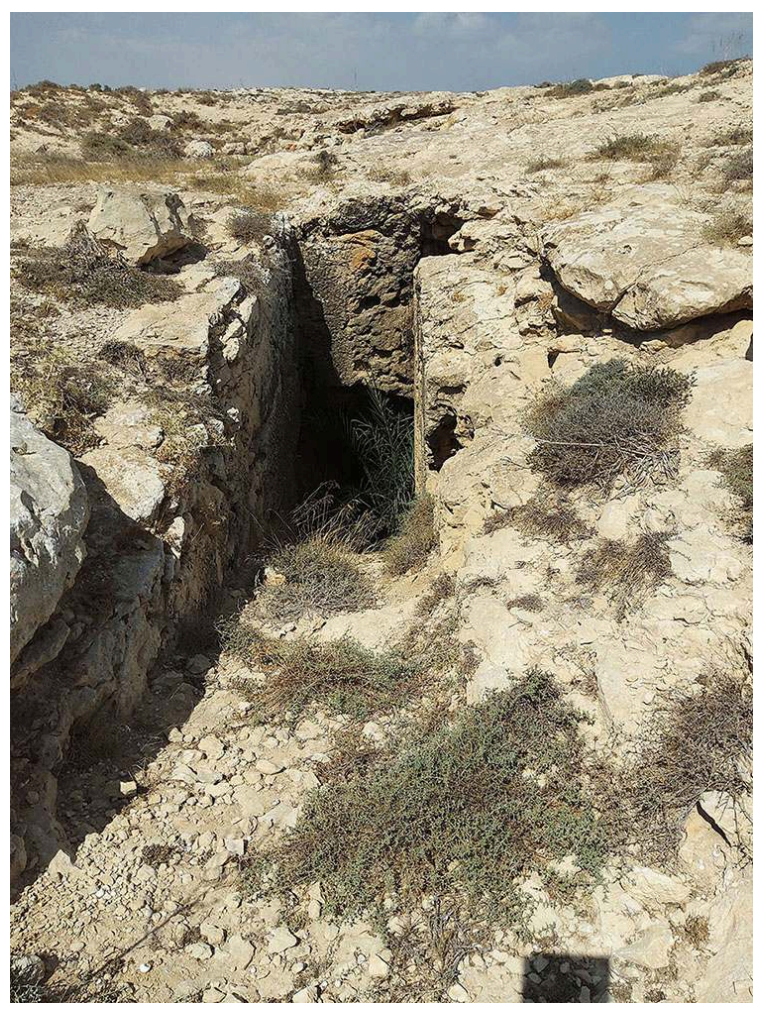

(C) Archives CEAlex. CEAlex_GMR_2020_NDMPF_009

\subsection{Présentation de sites prospectés : le secteur de Bahig}

\subsubsection{GMR0059 - Sidi Soliman el-Tayer}

(fig. 52)

Région de Naga el-Sanaqra 
Fig. 52. Carte archéologique de la Maréotide. Croquis du site GRM0059, Sidi Soliman el-Tayer : en bleu, limites actuelles du site et secteurs d'occupation; en vert, vestiges des karms sur le terrain ; en orange, anomalies (karm) visibles sur Corona 1968 (V. Pichot).

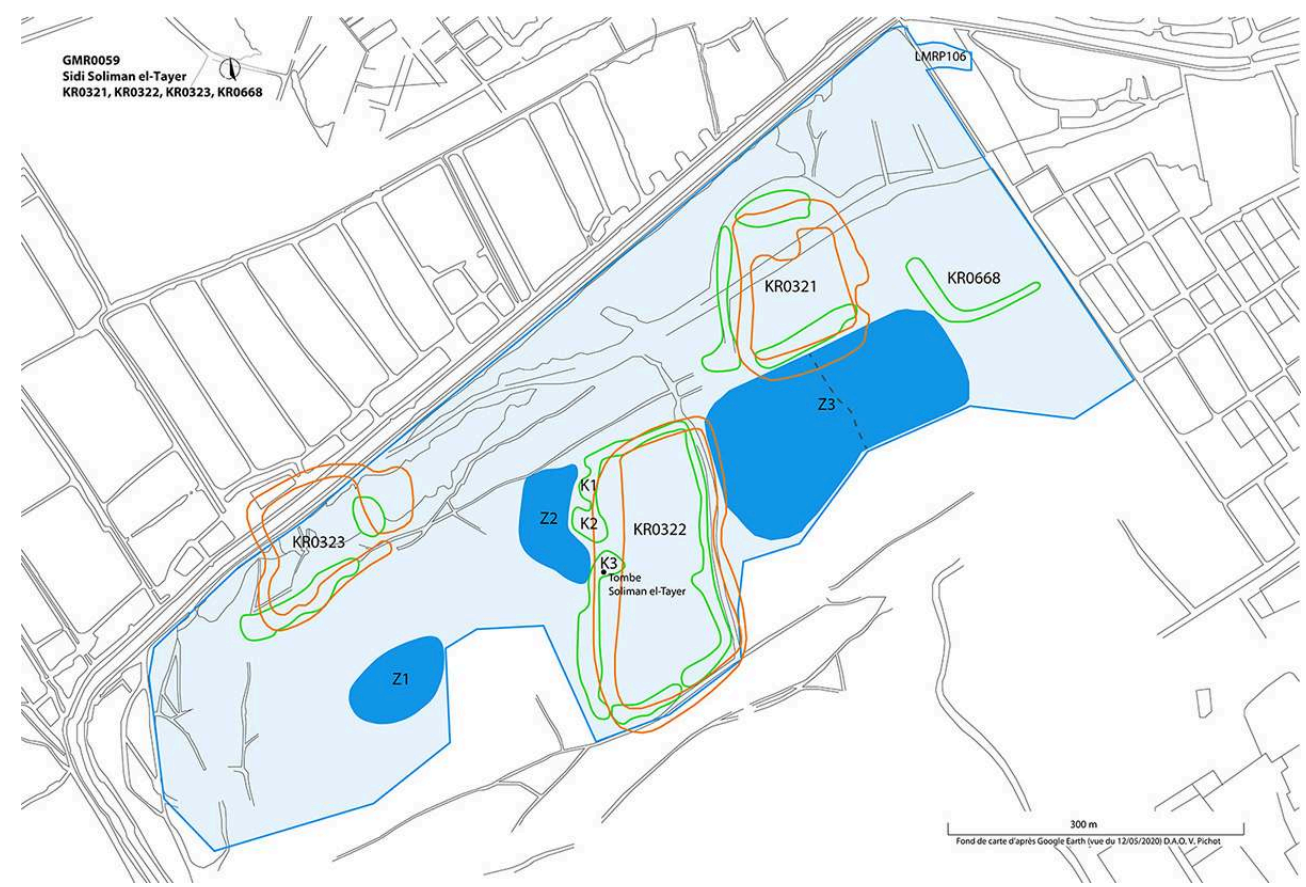

(C) Archives CEAlex. CEAlex_GMR_2020_NDMCN_004

À proximité immédiate de GMR0058 et à $1 \mathrm{~km}$ au nord de Kôm Bahig (GMR0067), ce site est déjà connu par les prospections menées par le LMRP22. Il s'agit d'un grand site d'une surface de plus de 7 hectares qui s'étend le long de la rive du lac sur $1,3 \mathrm{~km}$ en estouest. De nombreuses structures ont été répertoriées (sakiehs, bains, habitats divers). En 2019, nous avions ciblé trois karms (KR0321, KR0322, KR0323) repérés sur la carte du survey de 1940 et sur les cartes satellites. L'objectif était cette année de compléter la documentation de l'ensemble du site et d'effectuer quelques vérifications, notamment dans la partie orientale et sur la partie nord-ouest du karm central (KR0322).

$\mathrm{Au}$ nord-est, dans la partie basse du site, les prospections du LMRP avaient noté la présence d'une sakieh et les vestiges de murs de deux bâtiments (un au nord-est de la sakieh, un autre à l'est) (LMRP106). La céramique associée indiquait une occupation du $\mathrm{IV}^{\mathrm{e}} \mathrm{s}$. au VII ${ }^{\mathrm{e}}$ s. apr. J.-C. Aujourd'hui l'ensemble a totalement disparu.

Les vestiges d'un karm (KR0668) bien que peu lisibles ont été repérés cette année dans la partie orientale du site, portant à quatre le nombre de karms implantés sur le site : il s'agit des vestiges des talus ouest et sud totalement arasés que l'on suit chacun sur à peu près $100 \mathrm{~m}$ (fig. 53). 
Fig. 53. Carte archéologique de la Maréotide. GMR0059, karm (KR0668) situé dans la partie orientale du site, vestige du talus sud, vu depuis l'est (V. Pichot).

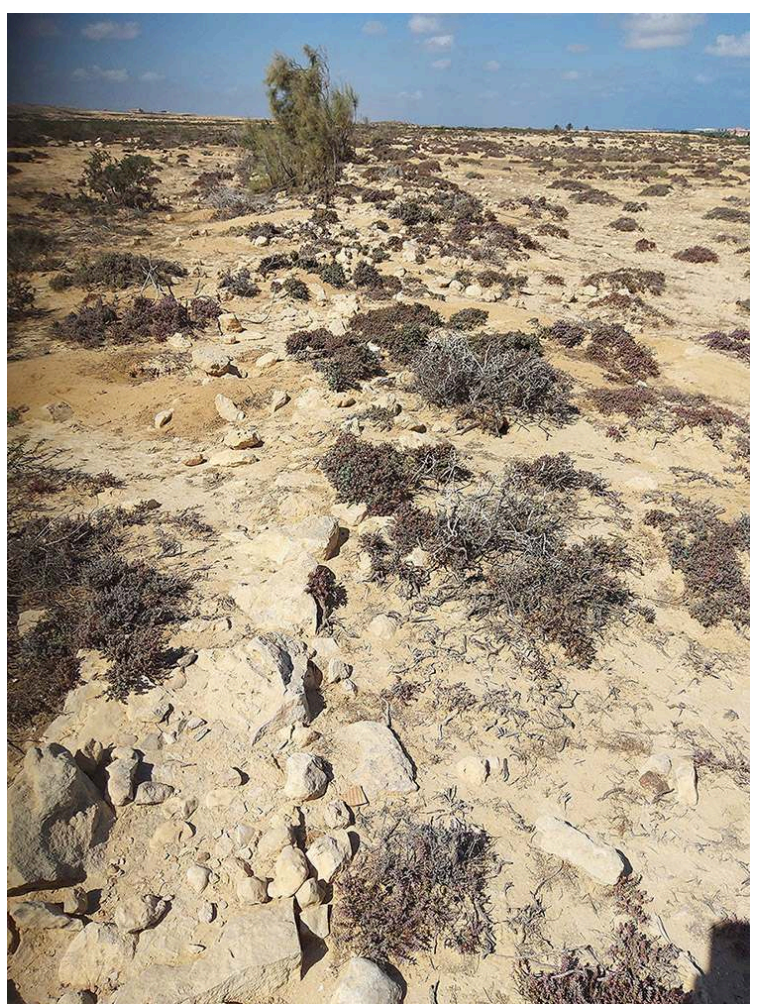

(c) Archives CEAlex. CEAlex_GMR_2020_NDMPF_010

Trois zones d'occupations ont été prospectées cette année. La première (Z1) située dans la partie occidentale du site est un promontoire rocheux qui ne livre aucune structure en surface mais une concentration de céramiques, reste d'une occupation du secteur. En surface de cette zone, quelques tessons très fragmentés et érodés ont été recueillis. Une forte concentration de matériel a été notée dans la partie centrale du promontoire (au nord). Il couvre une période chronologique large allant de la période ptolémaïque (amphore rhodienne) et du Haut-Empire (jatte à marli) à la période islamique (glaçures). La période romaine tardive et le début de la période islamique sont représentés de manière significative (LRA 1, LRA 5/6, LR D).

La deuxième zone d'occupation (Z2) située à $170 \mathrm{~m}$ plus à l'est, à proximité nord-ouest du karm central (KR0322), se compose de plusieurs kôms qui indiquent la présence de grands bâtiments et de structures à caractère hydraulique avec un bassin et peut-être une citerne (LMRP102). La présence de bains n'est pas à exclure. La céramique indique une occupation de la fin du $\mathrm{IV}^{\mathrm{e}} \mathrm{s}$. av. J.-C. jusqu'aux $\mathrm{VI}^{\mathrm{e}}-\mathrm{VII}{ }^{\mathrm{e}} \mathrm{s}$. apr. J.-C. L'occupation la mieux attestée est toutefois l'époque romaine tardive. On note à proximité du cimetière et de la tombe de Soliman el-Tayer, une zone qui livre des pierres brûlées et fragmentées ainsi que de la céramique surcuite (indices d'un bâtiment incendié ?) (fig. 54). 
Fig. 54. Carte archéologique de la Maréotide. GMR0059, zone de pierres brûlées et fragmentées à l'ouest du karm KR0322, vue depuis le sud - sud-est, à l'arrière-plan à droite on voit la proéminence K2 (V. Pichot).

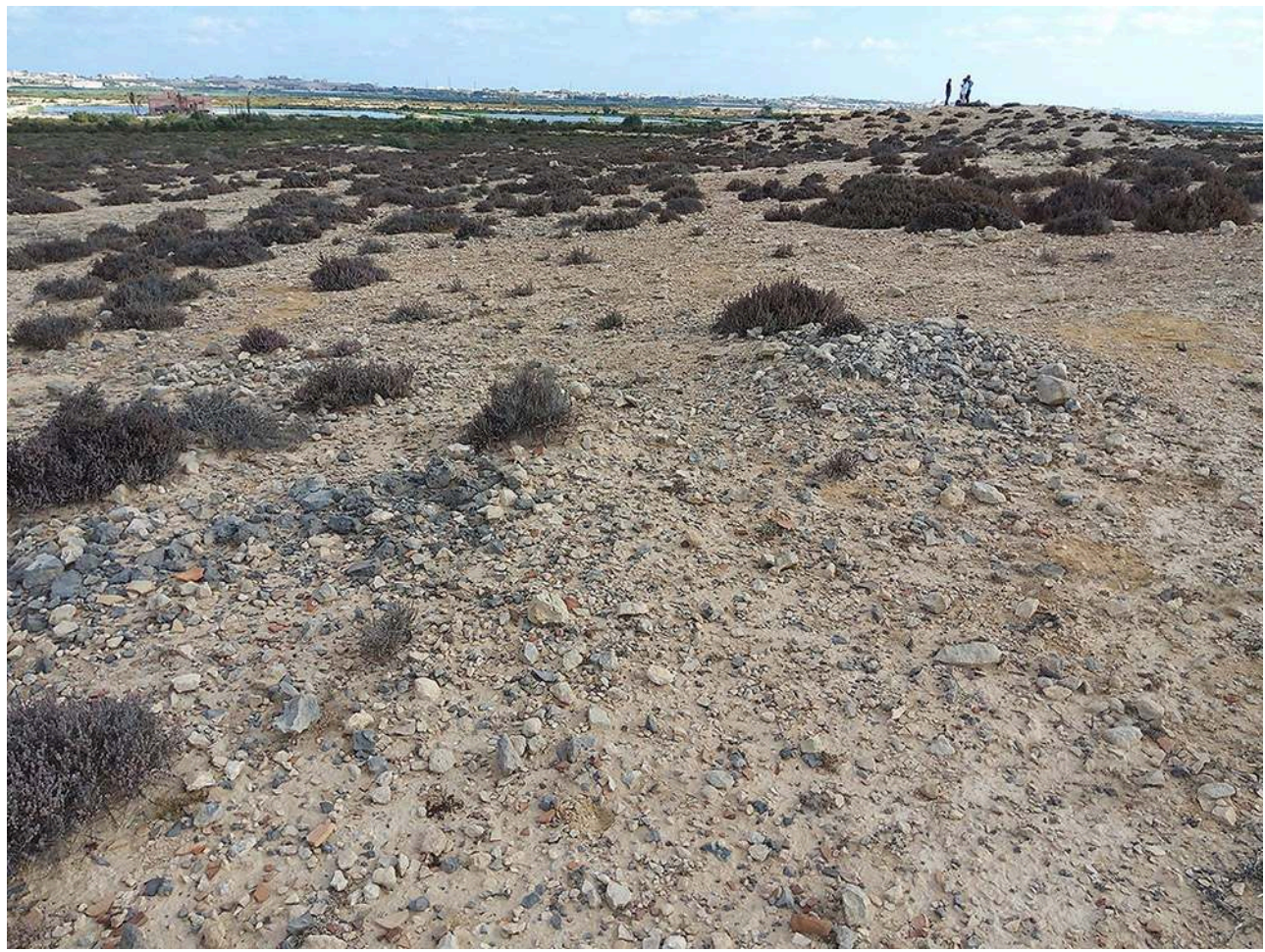

(c) Archives CEAlex. CEAlex_GMR_2020_NDMPF_011

Une vérification des proéminences formant la moitié nord du talus ouest du karm KR0322 a été menée lors de cette mission. Ces proéminences, dont deux s'imposent dans le paysage, indiquent la présence de bâtiments enfouis. La proéminence la plus au sud (K3, au centre du talus) est conservée en partie grâce à la présence de la tombe de Sidi Soliman el-Tayer (fig. 55) et d'un cimetière associé, et semble adossée au talus à l'intérieur de l'enceinte. 
Fig. 55. Carte archéologique de la Maréotide. GMR0059, vue à l'arrière-plan de la tombe de Sidi Soliman el-Tayer positionnée sur la partie sud de la proéminence $K 3$, depuis un ancien cimetière situé à l'ouest du karm KR0322 (V. Pichot).

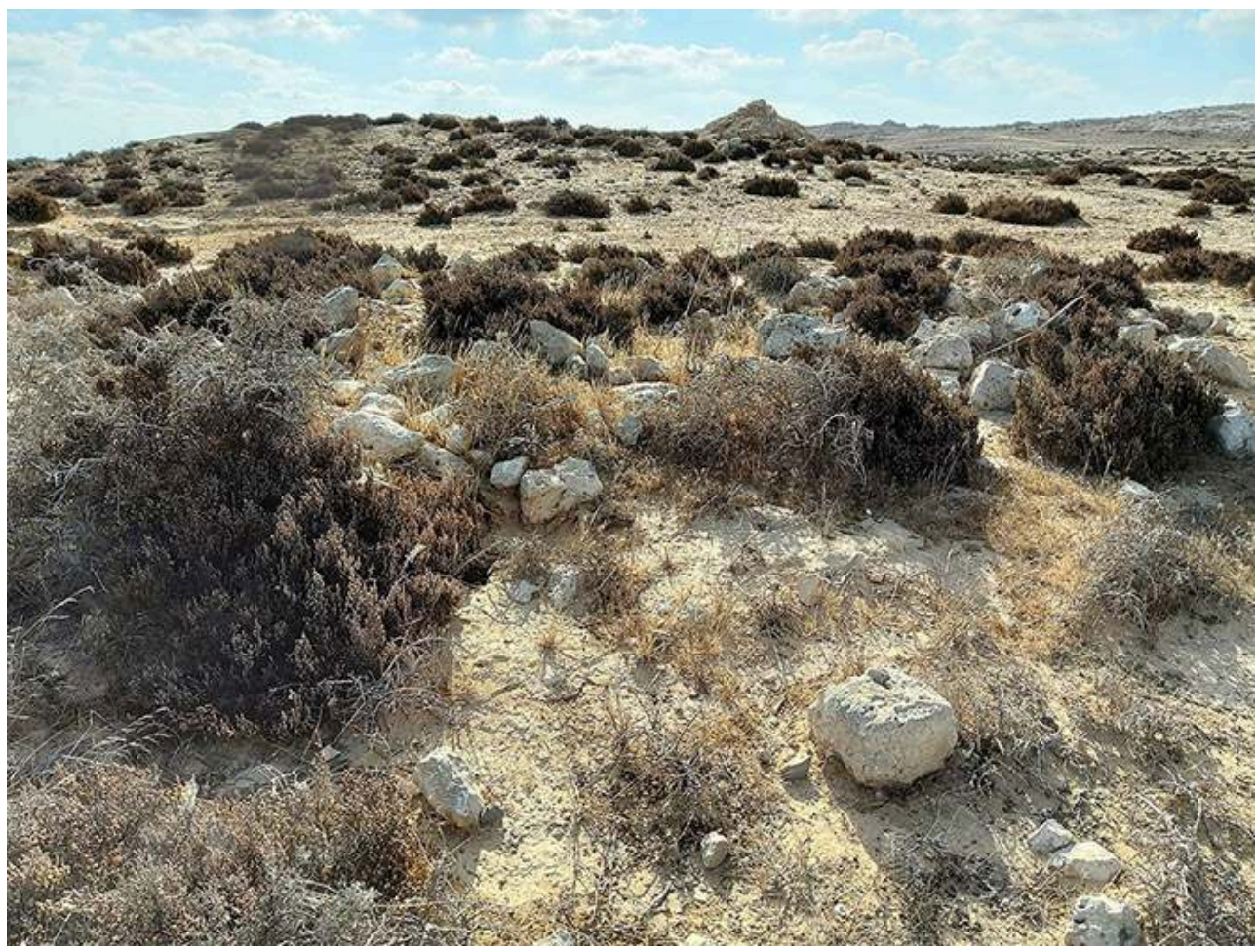

(c) Archives CEAlex. CEAlex_GMR_2020_NDMPF_012

La seconde proéminence (K2), à une vingtaine de mètres au nord de $\mathrm{K} 3$, semble s'adosser au talus à l'extérieur de l'enceinte. En surface de nombreux blocs épars (fig. 56) et surtout des alignements de gros blocs confirment la présence d'un bâtiment imposant. 
Fig. 56. Carte archéologique de la Maréotide. GMR0059, partie nord-ouest du talus occidental du karm KR0322, vue depuis le nord : au premier plan, proéminence $K 1$, au second plan, proéminence K2 (V. Pichot).

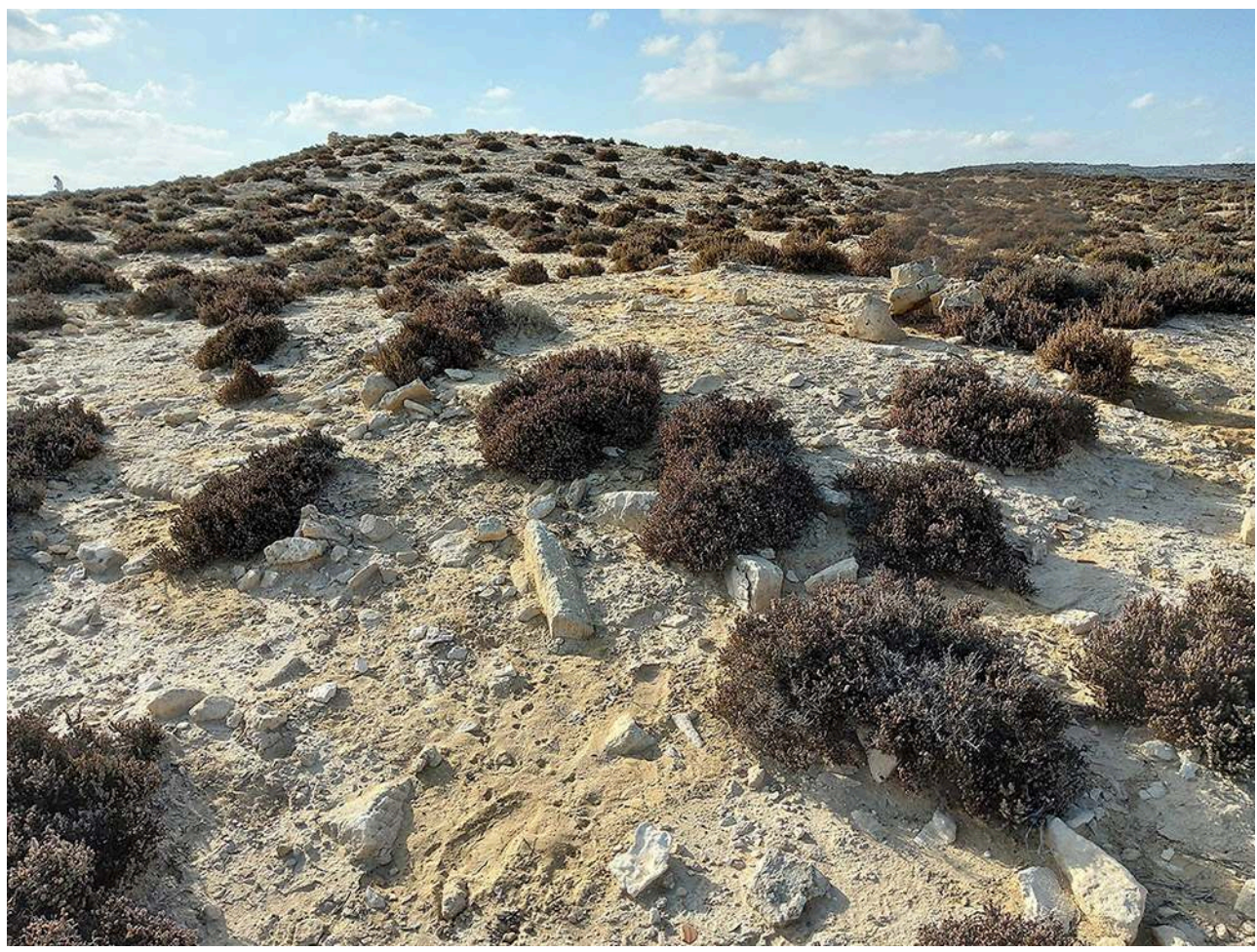

(c) Archives CEAlex. CEAlex_GMR_2020_NDMPF_013

76 L'angle nord-ouest du karm (K1) (voir fig. 56-57) montre en surface de nombreux murs dont certains très épais qui indiquent la présence de bâtiments imposants dans ce secteur. Le mobilier vu en surface lors de la prospection de 2019 indique une occupation du Haut-Empire (nombreux fragments d'AE 3) et de l'époque romaine tardive (anses de LRA 1 et $L R \mathrm{D}$ ).

77 Ces trois proéminences présentent en 2020 un matériel de surface abondant sous forme de gros fragments peu érodés. La période romaine tardive et le début de l'époque islamique sont les mieux attestés ( $L R A 1, L R A 5 / 6, L R$ D, African Red Slip Ware, céramiques du groupe $\mathrm{K}, L R C)$, toutefois, de nombreux fragments datés des périodes ptolémaïque ( $\mathrm{AE} 2$, amphore rhodienne) et romaine (marmite, amphore crétoise) ont également été identifiés en surface. 
Fig. 57. Carte archéologique de la Maréotide. GMR0059, angle nord-ouest du karm KR0322, proéminence $K 1$ vue depuis le sud-est (A. Simony).

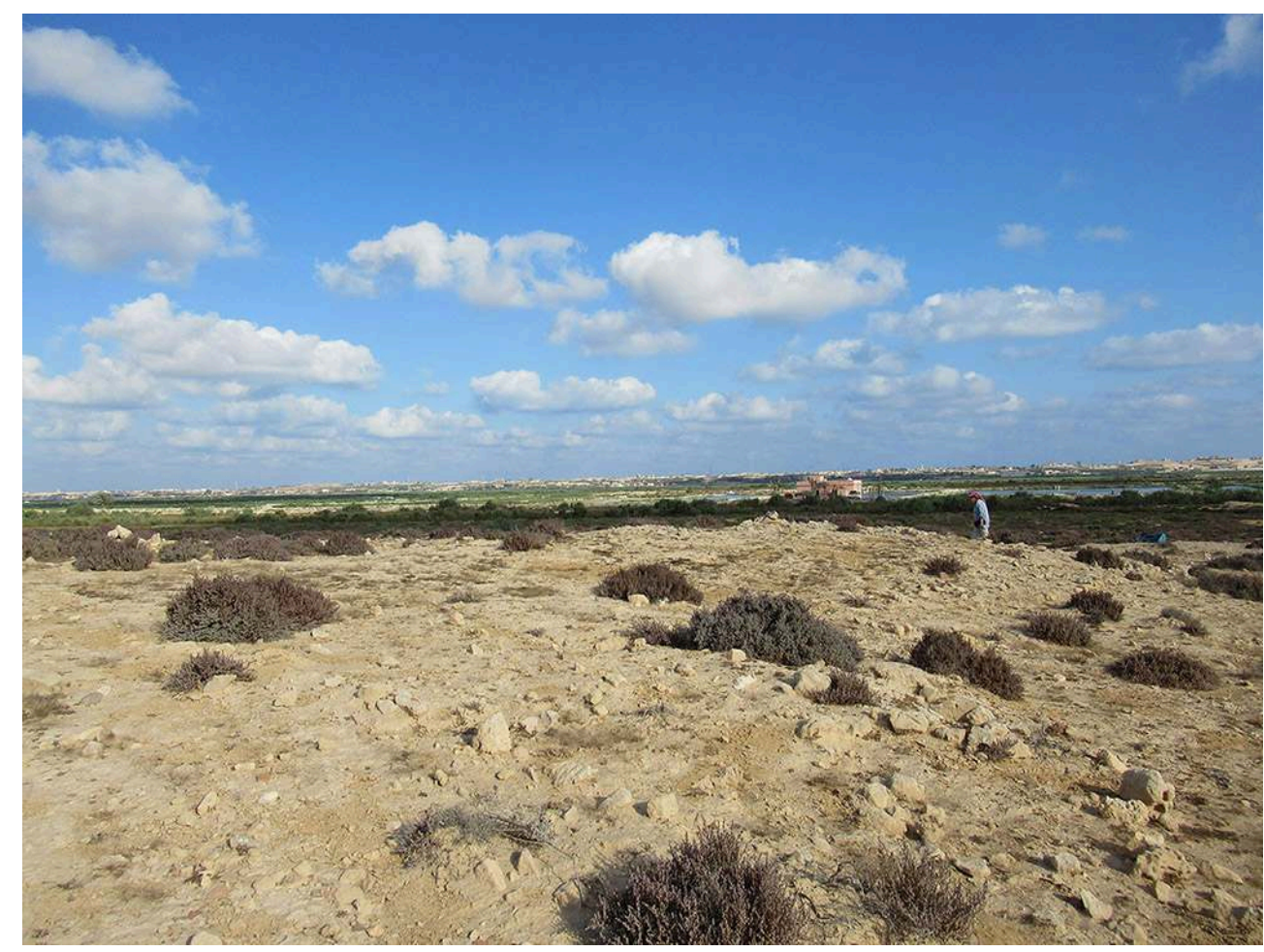

(C) Archives CEAlex. CEAlex_GMR_2020_NDMPF_014

La troisième zone d'occupation (Z3) se situe directement à l'est du karm central (KR0322) et est limitée au nord par le karm KR0321 et à l'est par le karm KR0668. Il s'agit d'une zone de $200 \mathrm{~m}$ nord-sud sur plus de $350 \mathrm{~m}$ est-ouest. La moitié occidentale est la mieux préservée: de petites proéminences, témoins de bâtiments enfouis, rythment le paysage; de nombreux alignements de blocs et d'enduit sont visibles en surface dans le sédiment pulvérulent (résultat de l'effondrement de murs en briques crues) (fig. 58). L'ensemble de cette zone est couvert de nombreux blocs épars, de fragments d'enduit et de céramique. Le matériel céramique de surface, très fragmenté, couvre pourtant une chronologie large de la période ptolémaïque (bol convexe, AE 2), du Haut-Empire ( $\mathrm{AE} 3$, jatte à marli) et de la période romaine tardive et du début de la période islamique (LRA 1, LRA 5/6, LR D). 
Fig. 58. Carte archéologique de la Maréotide. GMR0059, vue d'une partie de la zone d'occupation (Z3) qui se développe à l'est du karm KR0322, vue depuis le sud (V. Pichot).

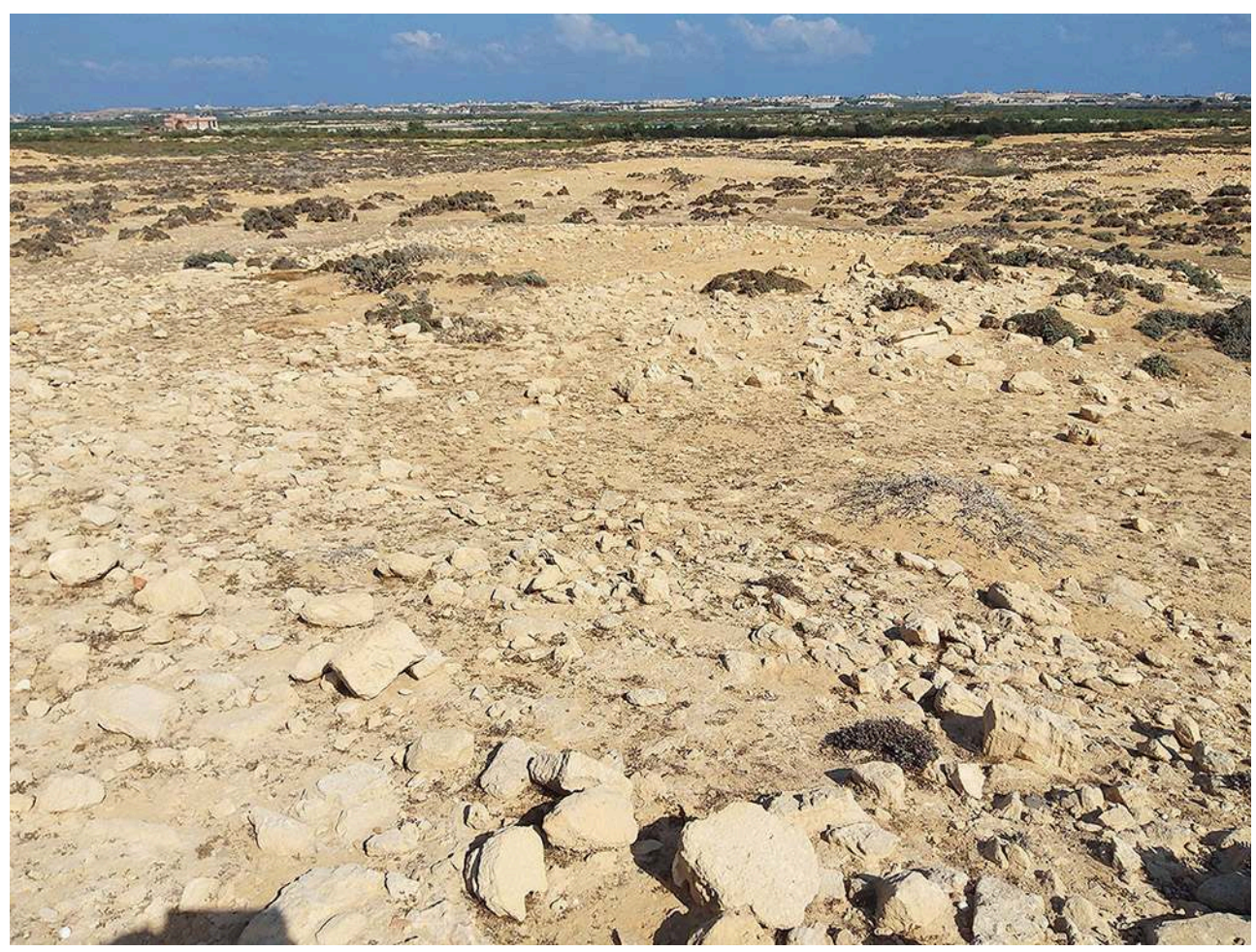

(c) Archives CEAlex. CEAlex_GMR_2020_NDMPF_015

L'occupation de l'ensemble du site est large. Notre expertise céramologique confirme la proposition du LMRP d'un début d'occupation à la fin du $\mathrm{IV}^{\mathrm{e}}-\mathrm{III}^{\mathrm{e}} \mathrm{s}$. av. J.-C., mais étend l'occupation jusqu'aux $\mathrm{VII}^{\mathrm{e}}-\mathrm{VIII}{ }^{\mathrm{e}}$ s. apr. J.-C., avec une occupation plus dense à l'époque romaine tardive. L'occupation en bordure du lac semble datée plus particulièrement de l'époque hellénistique et du Haut-Empire.

\subsubsection{GMR0133 - KR0333}

(fig. 59)

\section{Région de Bahig}


Fig. 59. Carte archéologique de la Maréotide. Croquis du site GMR0133 - KR0333 : en bleu limites actuelles du site ; en rouge, anomalies (karm) topographiées en 1914 ; en orange, anomalies (karm) topographiées en 1940 ; en jaune, anomalie (karm) visible sur Corona 1968 (V. Pichot).

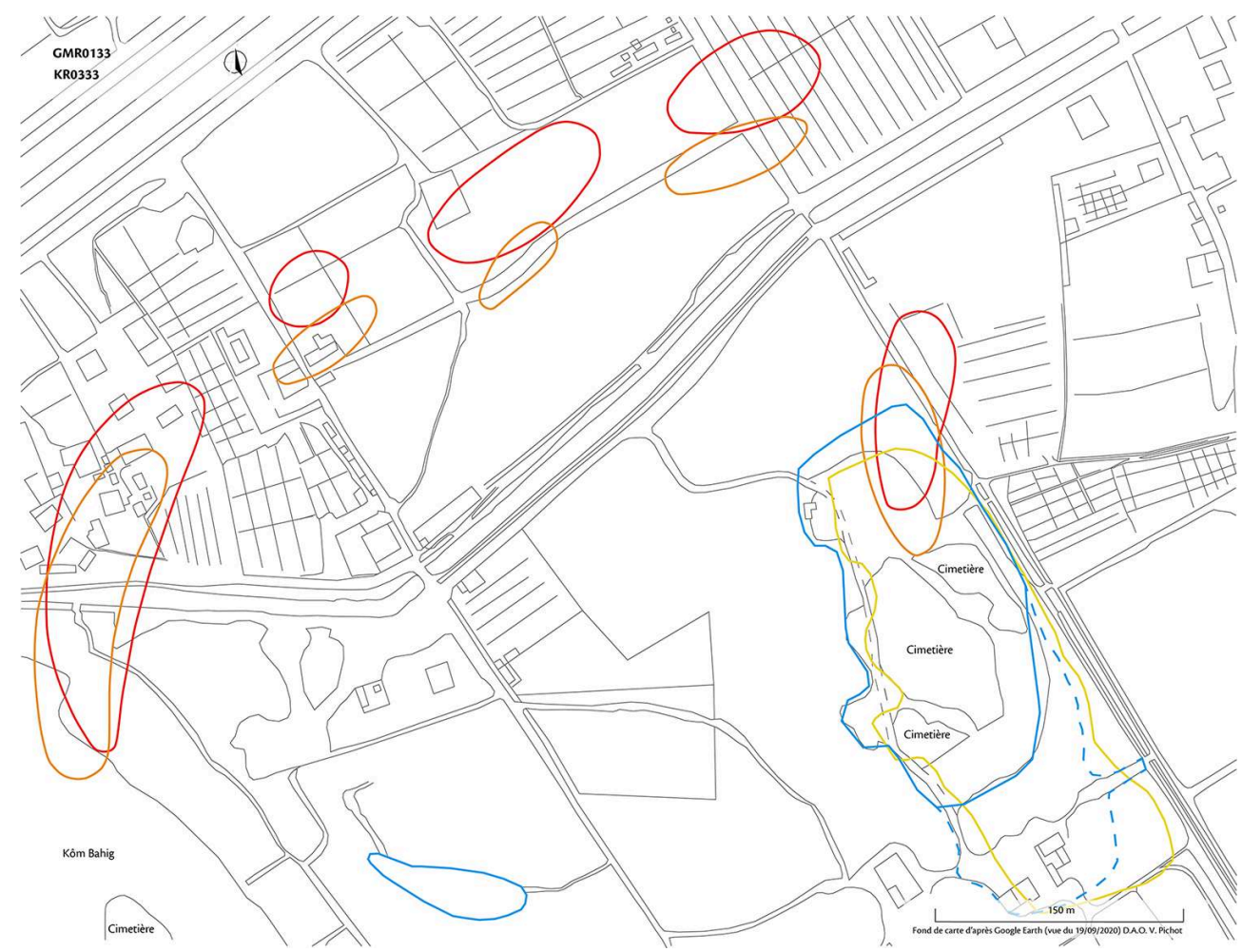

(C) Archives CEAlex. CEAlex_GMR_2020_NDMCN_005

À proximité nord-est de Kôm Bahig, ce site est une structure agricole, type karm, topographiée en partie sur les cartes anciennes (survey de 1914 et 1940) et en partie visible sur la carte satellite Corona de 1968. Elle est définie par des levées de terre en grande partie détruites : il subsiste une partie de la levée sud-ouest avec un petit kôm d'une longueur d'environ $100 \mathrm{~m}$ (direction nord-ouest - sud-est) et la levée orientale qui se compose d'un kôm d'environ $250 \mathrm{~m}$ de longueur, préservé grâce à la présence d'un cimetière dans sa partie centrale, et qui livre les vestiges de bâtiments probablement à fonction agricole installés sur la limite est du karm. Lors des prospections de 2017, seule la partie nord de la levée orientale avait montré un ensemble de bâtiments et du mobilier de la fin de la période hellénistique et du début du Haut-Empire (amphores rhodiennes et ES A). Lors des prospections de 2020 cette zone recouverte de végétation était peu lisible. En revanche, la partie sud de la levée orientale a livré en surface des alignements de blocs et d'enduit, vestiges de plusieurs bâtiments (fig. 60). 
Fig. 60. Carte archéologique de la Maréotide. GMR0133, vestige de la levée orientale du karm KR0333 : exemple d'alignement de blocs présent sur la partie sud, vu depuis l'ouest (V. Pichot).

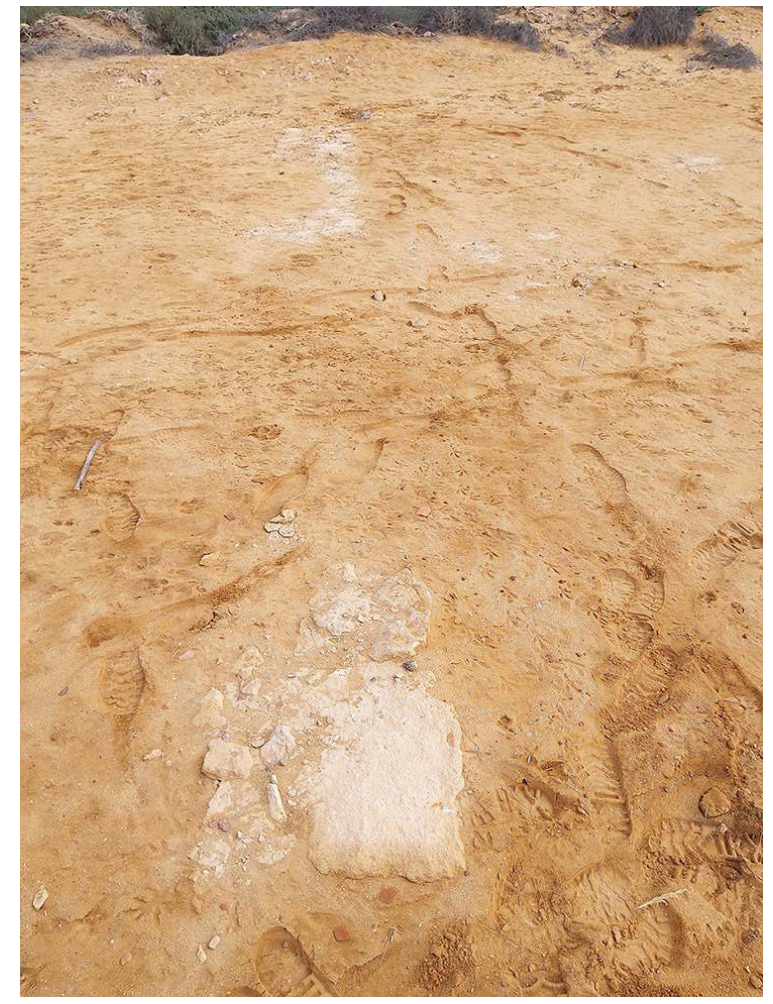

(C) Archives CEAlex. CEAlex_GMR_2020_NDMPF_016

81 Le mobilier indique pour cette zone une datation comprise entre la période ptolémaïque (amphore gréco-italique) et l'époque romaine tardive (amphores LRA 1, jattes rubanées, calices à encens).

\subsubsection{GMR0169 - KR0326}

\section{Région de Bahig}

Située à ca 750 m à l'est de Kôm Bahig, une anomalie de terrain de forme rectangulaire (ouverte à l'est) a été repérée sur les cartes satellites et correspondrait à un karm (KR0326) partiellement topographié (partie sud-est de l'enceinte) sur la carte du survey de 1940. Le sédiment est une argile orange typique des talus de karm dans la région. La superposition de la limite des vestiges sur la carte de 1940 montre qu'il s'agit d'une portion du talus oriental du karm originel qui a été creusé dans son épaisseur et arasé lors de la mise en culture de la zone, donnant à l'anomalie une forme de rectangle ouvert à l'est. D'autres vestiges de ce karm très arasé ont été repérés plus au sud (champ d'oliviers). La superposition de la limite de ce secteur sur la carte de 1940 montre qu'il s'agit, d'une part, de l'angle sud-est du karm et, d'autre part, d'une portion de la limite sud de l'enceinte. La zone de l'angle sud-est a été fortement décaissée et ne montre aujourd'hui que de maigres traces du talus de l'enceinte. À l'ouest, de l'autre côté de la piste, deux zones de petites tailles composées d'un sédiment argileux orangé sont les derniers témoins du talus sud de l'enceinte. L'ensemble a été fortement aplani : 
une habitation est installée actuellement sur la zone ouest tandis que la zone à l'est sert de zone de travail : battage, tri (dattes, olives).

Aucun mobilier et aucune structure autre que les talus des karms n'ont été répérés en surface dans ce secteur.

\subsubsection{GMR0170 - KR0052}

(fig. 61)

\section{Région de Bahig}

Fig. 61. Carte archéologique de la Maréotide. Croquis du site GMR0170 - KR0052 : en bleu, vestiges du karm sur le terrain ; en rouge, anomalie (karm) topographiée en 1940 ; en jaune, anomalie (karm) visible sur l'image satellite Landsat 1984 ; en fuschia, vestiges du promontoire livrant des restes d'occupation ; en orange, anomalie (promontoire) topographiée en 1940 (V. Pichot).

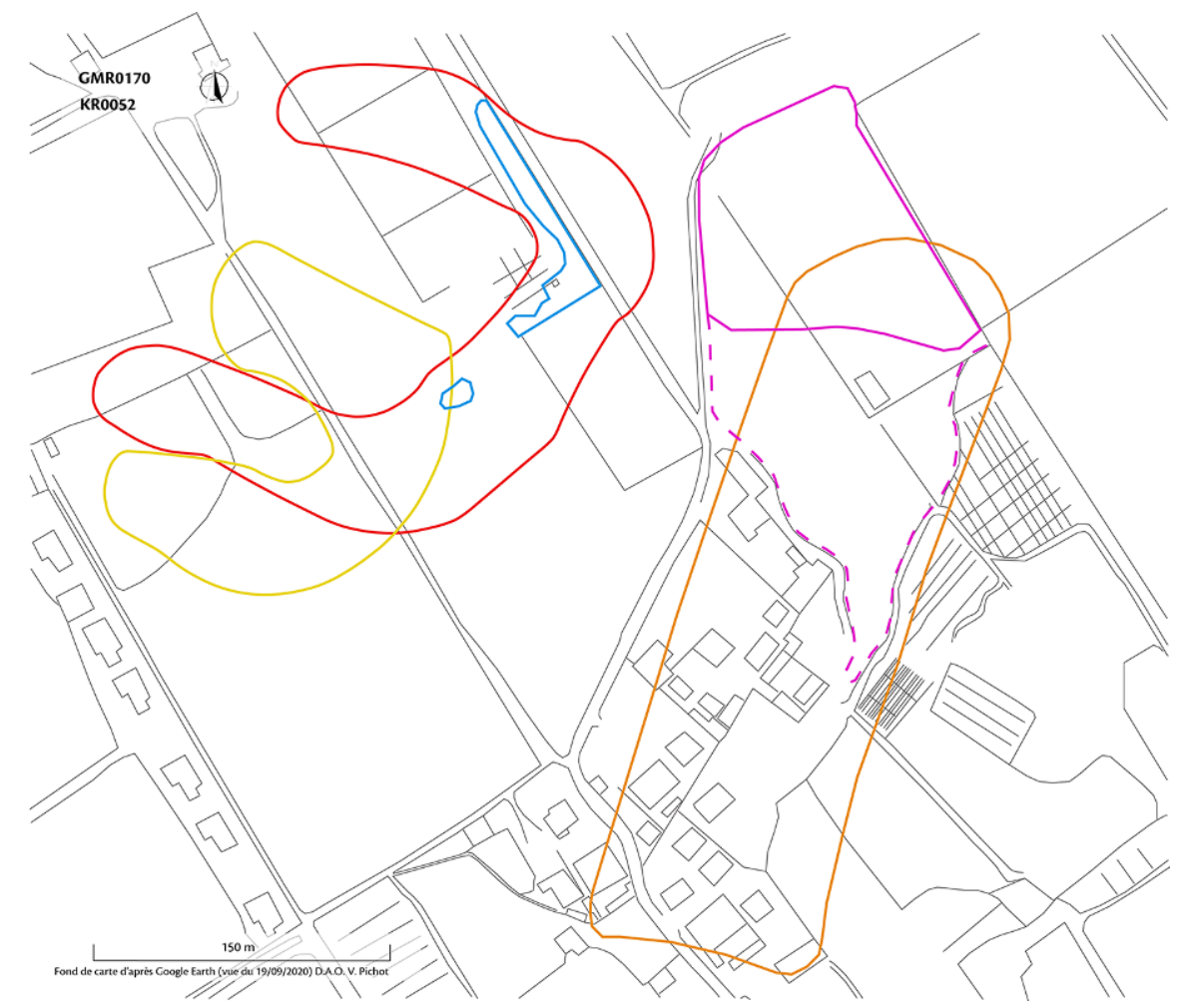

(C) Archives CEAlex. CEAlex_GMR_2020_NDMCN_006

Situé à ca $740 \mathrm{~m}$ à l'est de Kôm Bahig, ce site correspond à un karm (KR0052) topographié sur la carte du survey de 1940 et repéré sur l'image satellite Landsat 1984. Le karm rectangulaire (orientation nord-ouest - sud-est) a été très endommagé : sur le terrain les restes se composent de l'angle sud-est et d'une partie des talus est et sud. Le talus oriental apparaît sous la forme de monticules visibles surtout dans sa partie sud, le reste est aplani et en grande partie sous la végétation. Un fragment de céramique a été trouvé à la base du talus : il s'agit d'un bord de jatte à marli de production locale daté du Haut-Empire. La partie sud de l'enceinte (visible sur les images satellites Google Earth) est divisée en deux par une piste. Sur le terrain le talus a presque totalement disparu excepté quelques monticules dans la partie orientale. 
85 À l'est de la route asphaltée, on note la présence d'un promontoire rocheux (fig. 62) qui a récemment été en partie arasé et détruit pour de futures constructions. Ce secteur livre en surface de rares fragments de céramique témoignant d'une occupation à mettre en relation avec le karm proche. Ils sont datés du Haut-Empire (amphore crétoise) et de la période romaine tardive au début de la période islamique (amphores LRA 1).

Fig. 62. Carte archéologique de la Maréotide. GMR0170, promontoire rocheux situé à l'est du karm KR0052, partie au sud-est intacte, vue depuis l'est (A. Simony).

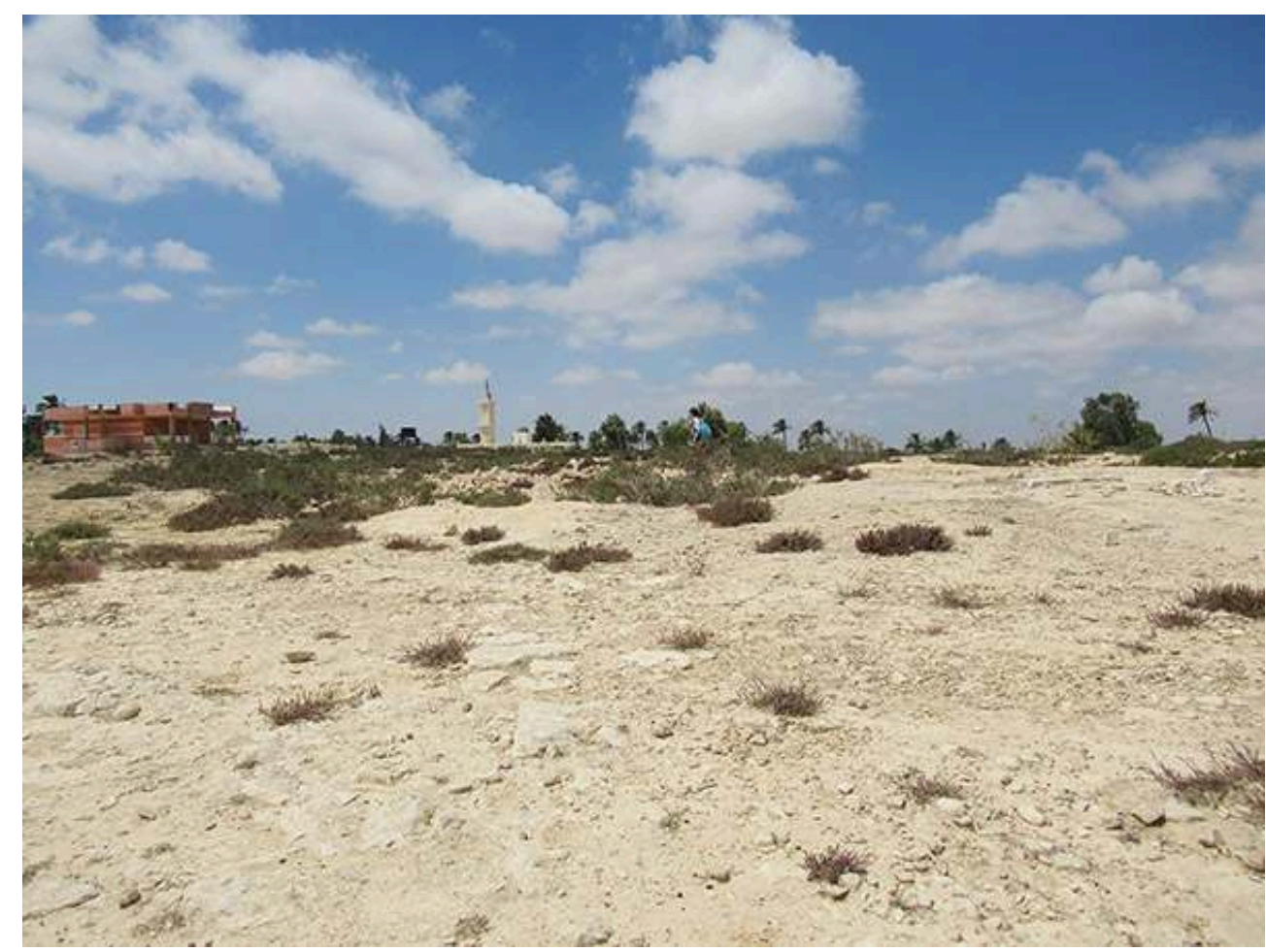

(c) Archives CEAlex. CEAlex_GMR_2020_NDMPF_017

\subsection{Présentation de sites prospectés : le secteur de Borg el-Arab}

\subsubsection{GMR0016 - Sidi Mahmoud}

(fig. 63)

Région de Borg el-Arab 
Fig. 63. Carte archéologique de la Maréotide. Croquis du site GMR0016 - Sidi Mahmoud : en bleu, limites actuelles du site ; en bleu (contour et fond), vestiges du talus du karm KR0137; en vert fouilles du MoTA en 1982-1983 et mission archéologique de P. Grossmann en 1992 ; en rouge, anomalies (karm) topographiées en 1914 ; en jaune, anomalies (karm) topographiées en 1920 ; en orange, anomalies (karm) topographiées en 1940 (V. Pichot).

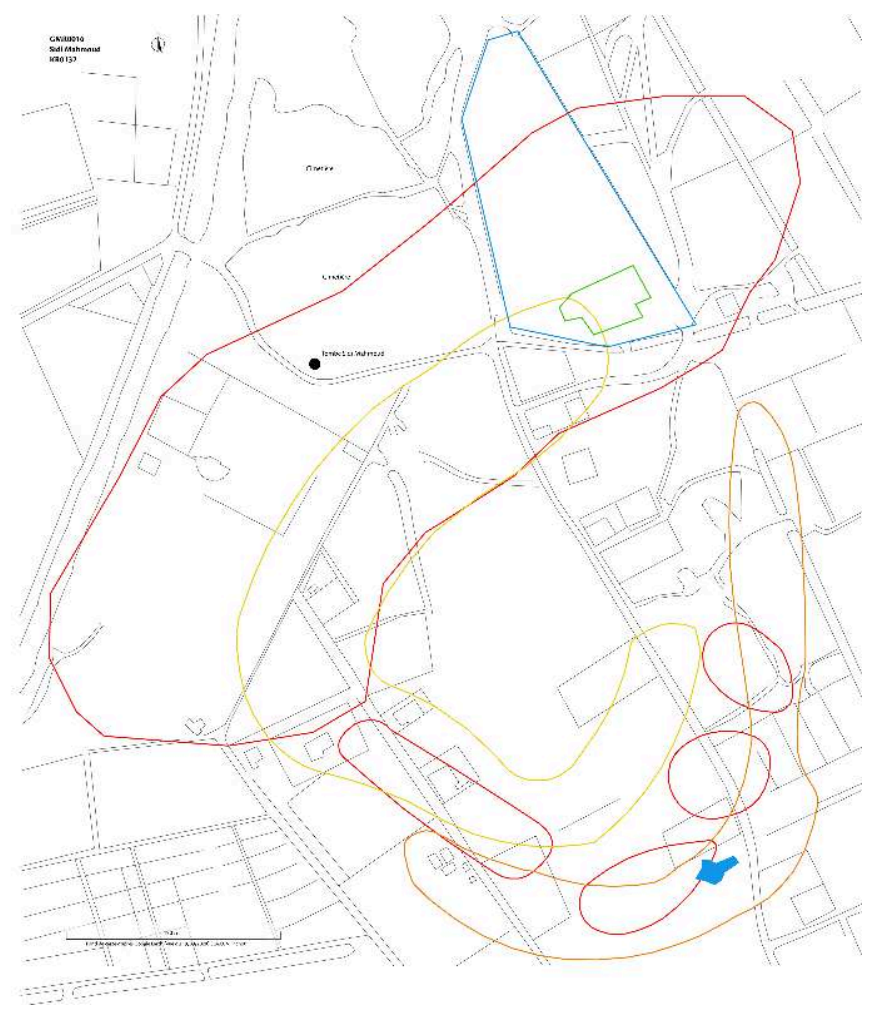

(c) Archives CEAlex. CEAlex_GMR_2020_NDMCN_007

Au nord de Borg el-Arab, à proximité orientale d'un cimetière et du mausolée de Sidi Mahmoud, ce site est connu par des fouilles anciennes (fouilles du MoTA en 1982-1983 et mission archéologique de P. Grossmann en 199223) qui ont livré les vestiges d'une église et de deux petits bâtiments (habitat). Le site relevant du département des antiquités islamiques et coptes, nous n'y avons pas eu accès. La prospection 2020 avait pour but de valider la présence d'un karm (KR0137), topographié sur la carte de l'Atlas Lower Egypt 1914 et sur les cartes des survey 1920-1930 et 1940, orienté nord-est - sudouest et qui se développait au sud-ouest du site ; le site de Sidi Mahmoud occupant une partie du talus nord de ce karm. La presque totalité du karm a été arasée et étalée, il reste néanmoins, à proximité de la route menant au site de Sidi Mahmoud, une butte de 3 à $4 \mathrm{~m}$ de haut, vestige du talus sud du karm (fig. 64). 
Fig. 64. Carte archéologique de la Maréotide. GMR0016, au second plan dernier vestige du talus sud du karm KR0137 à proximité de la route menant au site de Sidi Mahmoud, vu depuis le sud (V. Pichot).

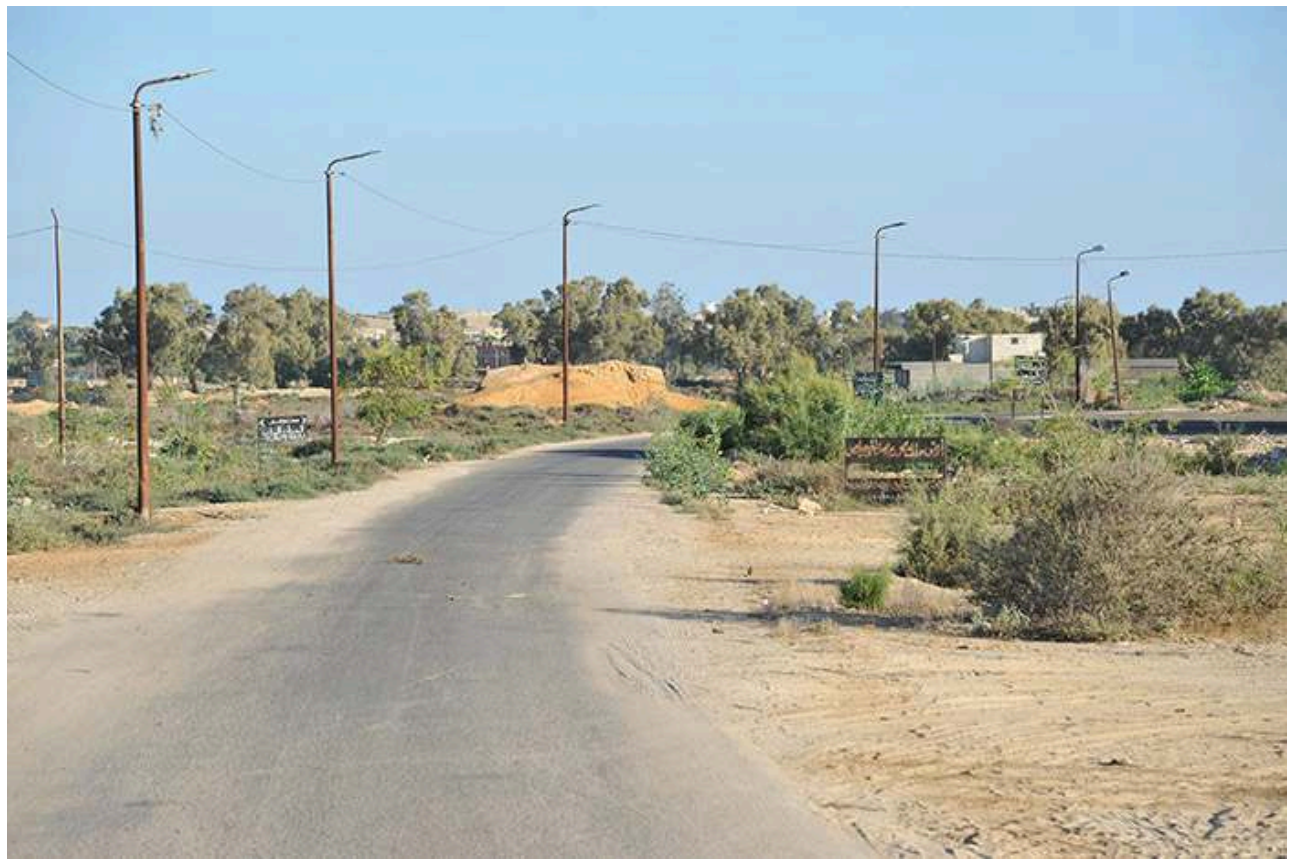

(c) Archives CEAlex. CEAlex_GMR_2020_NDMPF_018

\subsubsection{GMR0171 - Sidi Hamid Gaballah Soliman Shamata}

(fig. 65)

\section{Région de Naga el-Sanaqra}


Fig. 65. Carte archéologique de la Maréotide. Croquis du site GMR0171 - Sidi Hamid Gaballah Soliman Shamata : en bleu (contour et fond), limites actuelles du site; en rouge, anomalies (karms) topographiées en 1940 ; en orange, anomalies (karms) topographiées en 1914 ; en jaune, anomalie (KR507) repérée sur Landsat 1984 (V. Pichot).

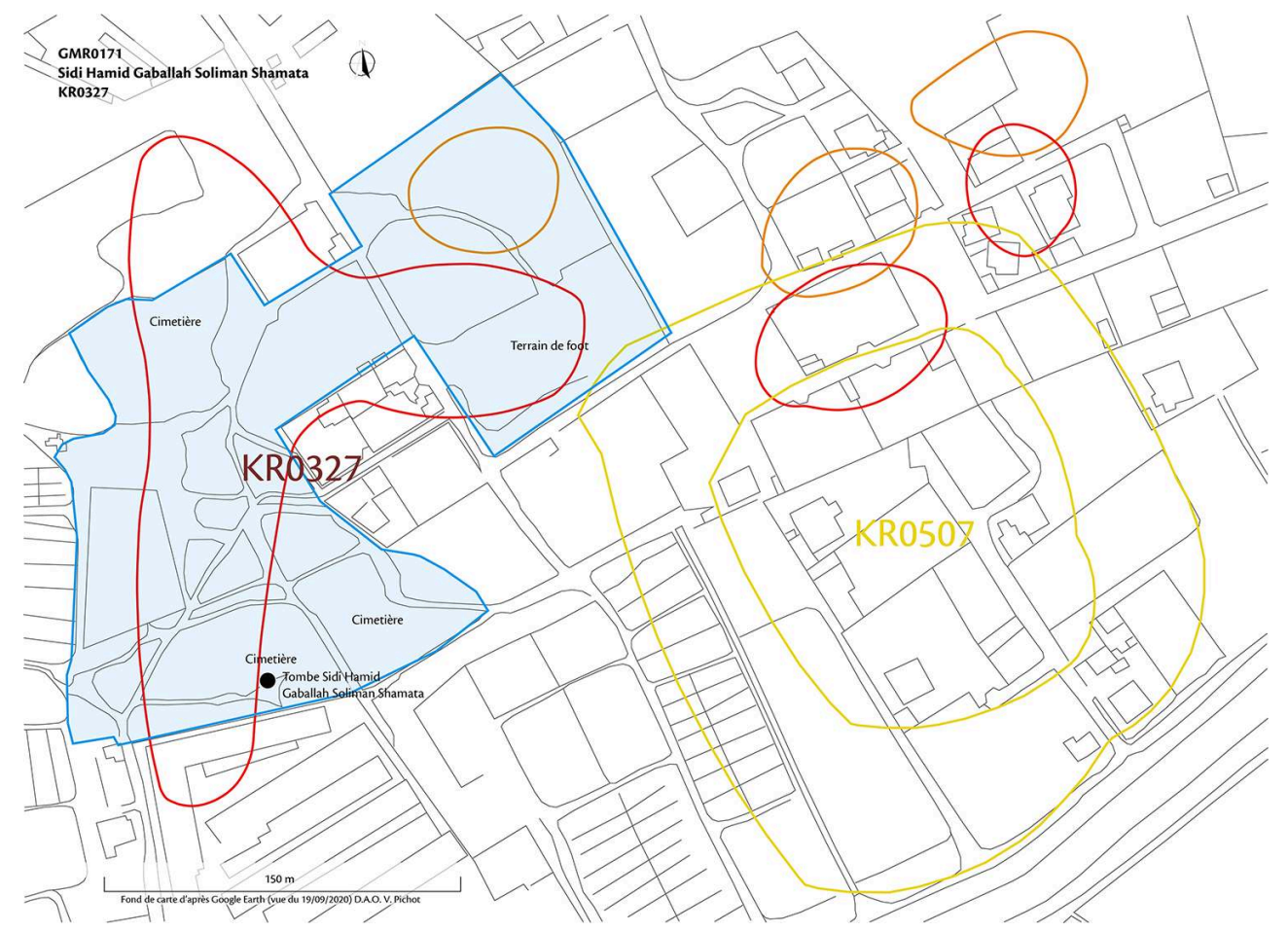

(c) Archives CEAlex. CEAlex_GMR_2020_NDMCN_008

Située entre la ride III et la ride IV à 1,5 km au nord-est de Borg el-Arab, une anomalie de terrain, repérée sur les images satellites Google Earth correspond aux restes d'un karm (KR0327) topographié sur la carte du survey de 1940. L'ensemble du secteur a été perturbé par la mise en culture et l'implantation de parcelles et le site est traversé par des pistes. L'ensemble est recouvert par un sédiment argileux jaune-orange caractéristique des karms de la région.

La partie nord du site est occupée par une légère proéminence qui correspond à une partie de la limite nord de l'enceinte cartographiée en 1940 dont la partie ouest a été décaissée et la partie orientale aplanie. Ce qui reste du talus du karm est occupé au nord-est par un cimetière. De nombreux blocs épars sont présents en surface (fig. 66). On distingue à certains endroits des départs d'alignements de blocs (murs) et d'alignements de mortier qui laissent supposer la présence de bâtiment(s). La limite ouest de l'enceinte cartographiée en 1940 n'est plus visible, décaissée en grande partie et aplanie à certains endroits. 
Fig. 66. Carte archéologique de la Maréotide. GMR0171, vestige du talus nord du karm KR0327, partie orientale vue depuis l'ouest, au second plan à gauche le cimetière (V. Pichot).

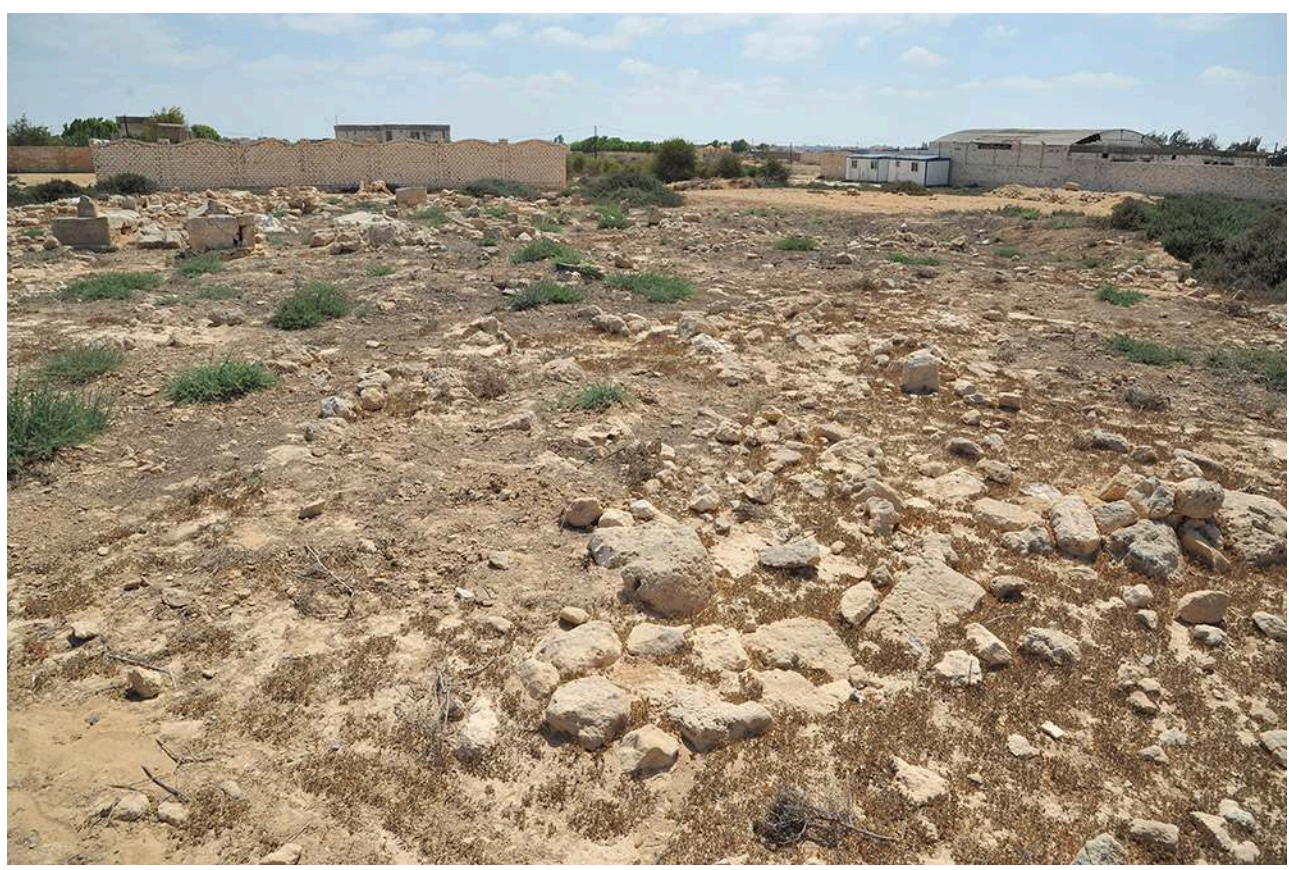

(C) Archives CEAlex. CEAlex_GMR_2020_NDMPF_019

89 À ca $20 \mathrm{~m}$ au sud de la proéminence nord, une zone qui a fait l'objet d'un léger décaissement, livre les vestiges de plusieurs bâtiments imposants (murs, grand nombre de blocs épars) (fig. 67-68).

Fig. 67. Carte archéologique de la Maréotide. GMR0171, secteur central du site, exemple de vestiges visibles en surface, vu depuis le nord-est (V. Pichot).

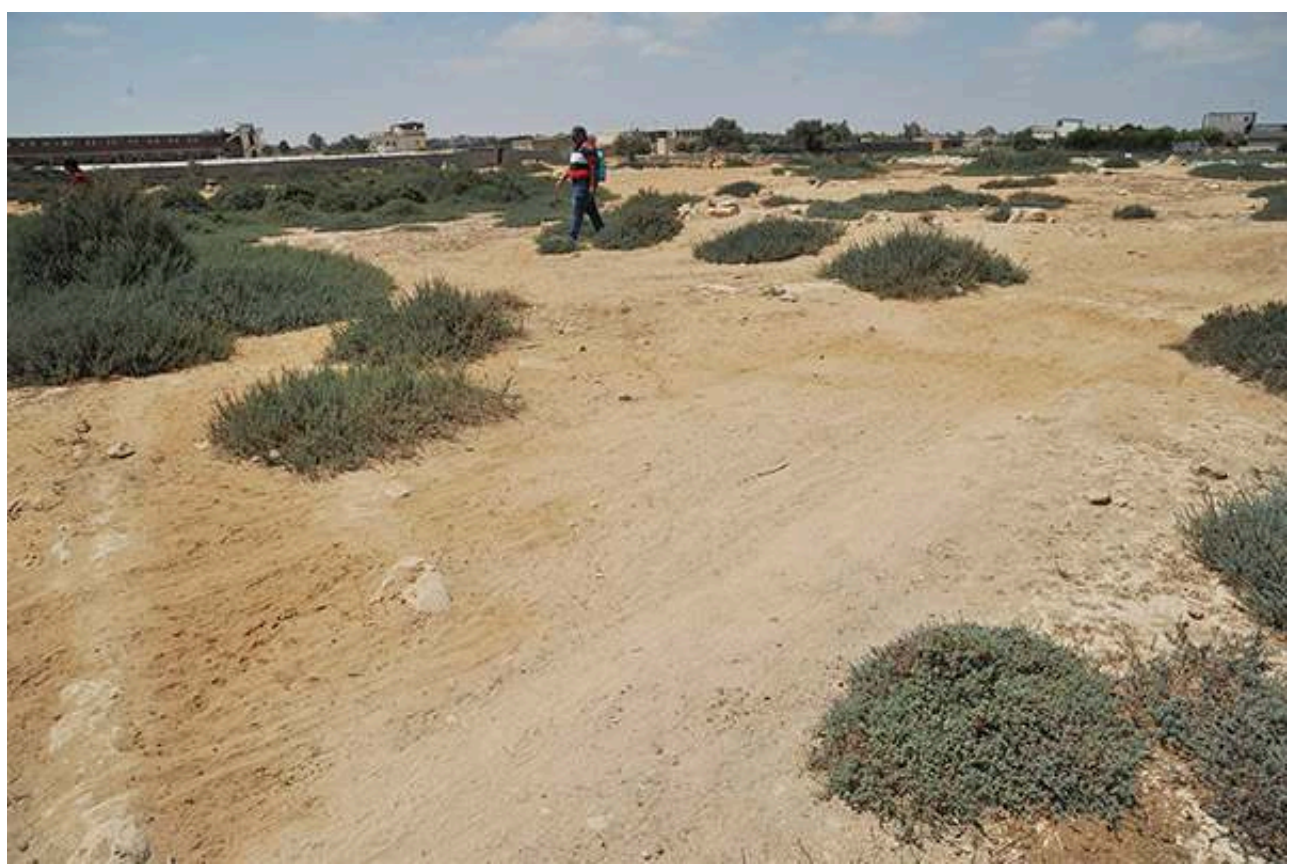

(c) Archives CEAlex. CEAlex_GMR_2020_NDMPF_020 
Fig. 68. Carte archéologique de la Maréotide. GMR0171, secteur central du site, exemple de vestiges visibles en surface, vu depuis le sud-est (A. Simony).

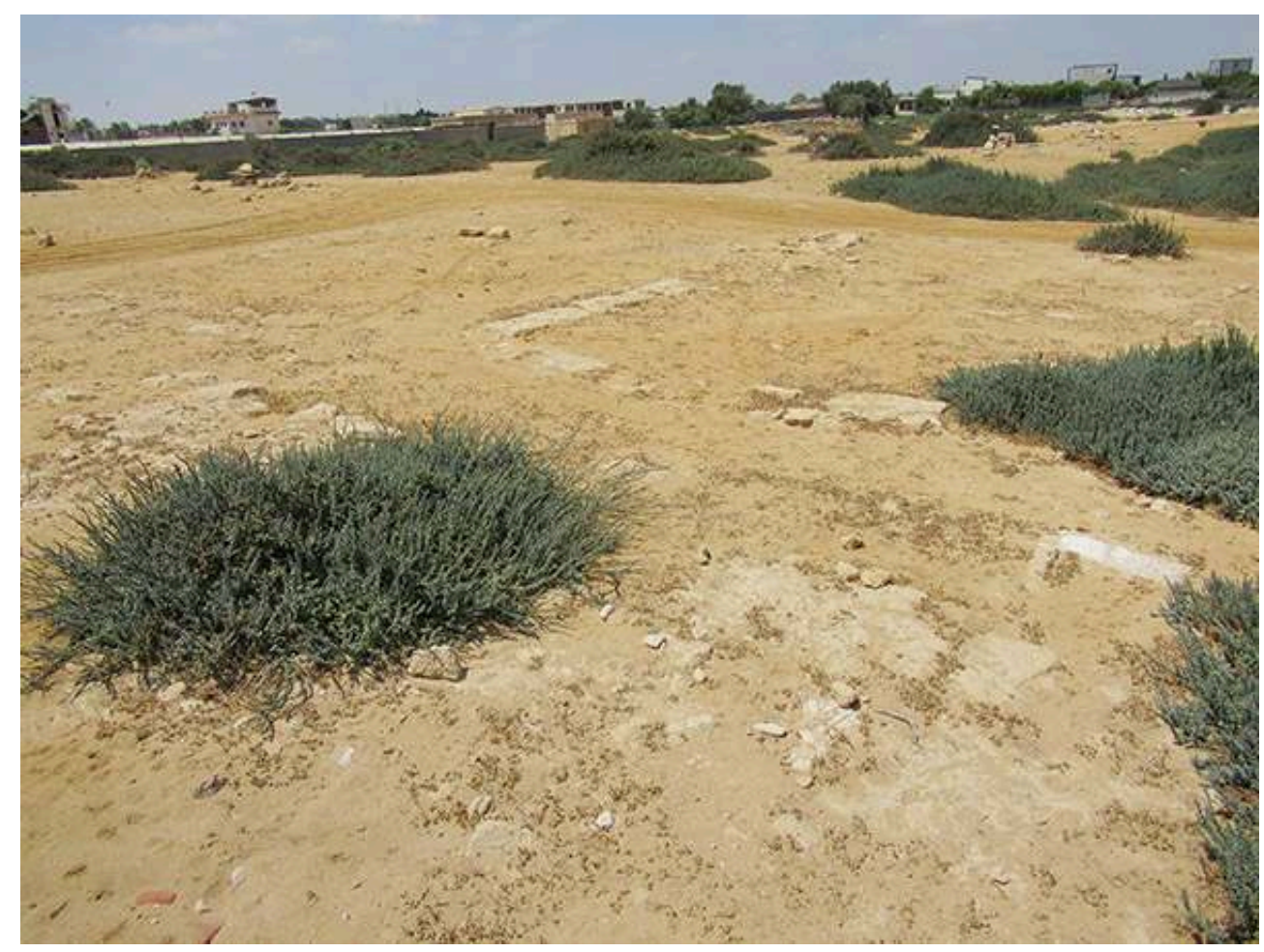

(c) Archives CEAlex. CEAlex_GMR_2020_NDMPF_021

90 Au sud-est du site, une proéminence, dont le sommet est occupé en partie par un cimetière (fig. 69), est un vestige de la limite sud de l'enceinte du karm à proximité de l'angle sud-est. Là aussi des blocs épars sont visibles en surface. 
Fig. 69. Carte archéologique de la Maréotide. GMR0171, vestige du talus sud du karm KR0327, vu depuis l'ouest, au second plan le cimetière moderne (V. Pichot).

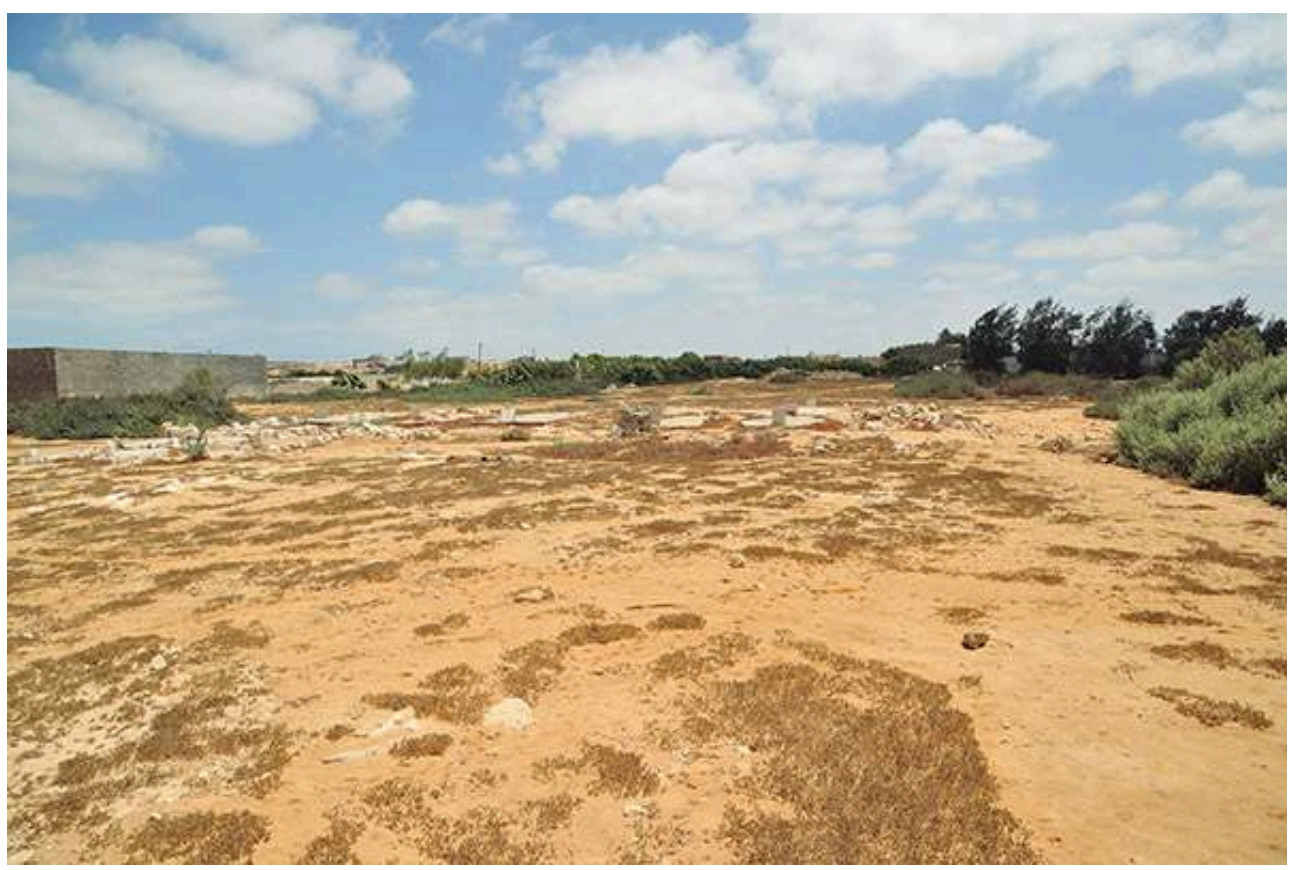

(C) Archives CEAlex. CEAlex_GMR_2020_NDMPF_022

91 À l'est du cimetière le site continue sur quelques dizaines de mètres : il pourrait s'agir d'une partie de l'angle sud-est du karm. La partie sud, totalement décaissée pour la mise en culture, offre une stratigraphie dans laquelle le soubassement du sol d'une structure (bassin, fouloir ?) est visible (fig. 70-71).

Fig. 70. Carte archéologique de la Maréotide. GMR0171, limite sud-est du site vue depuis le sudouest, stratigraphie occasionnée par un décaissement de terrain (V. Pichot).

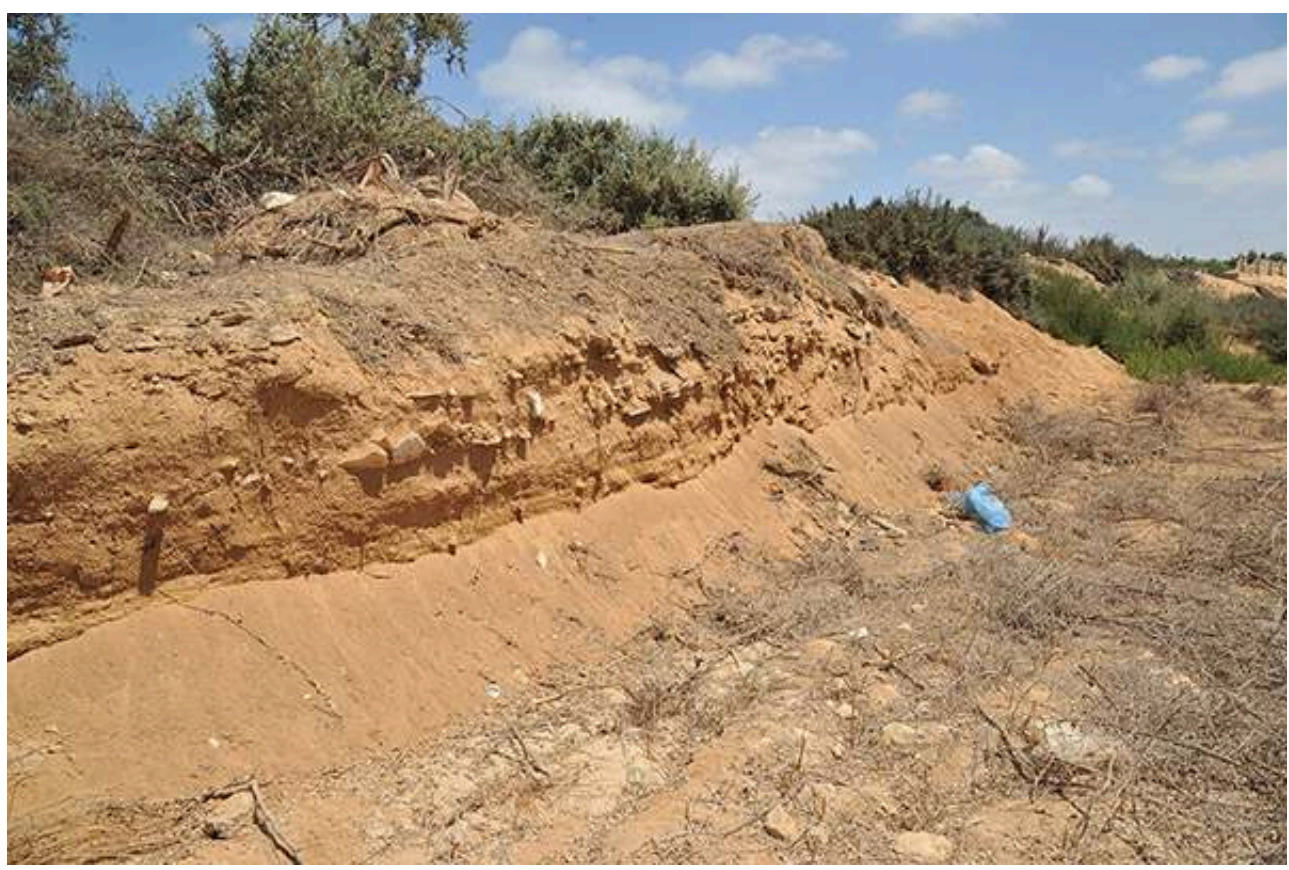

(C) Archives CEAlex. CEAlex_GMR_2020_NDMPF_023 
Fig. 71. Carte archéologique de la Maréotide. GMR0171, limite sud-est du site vue depuis le sud, stratigraphie occasionnée par un décaissement de terrain (V. Pichot).

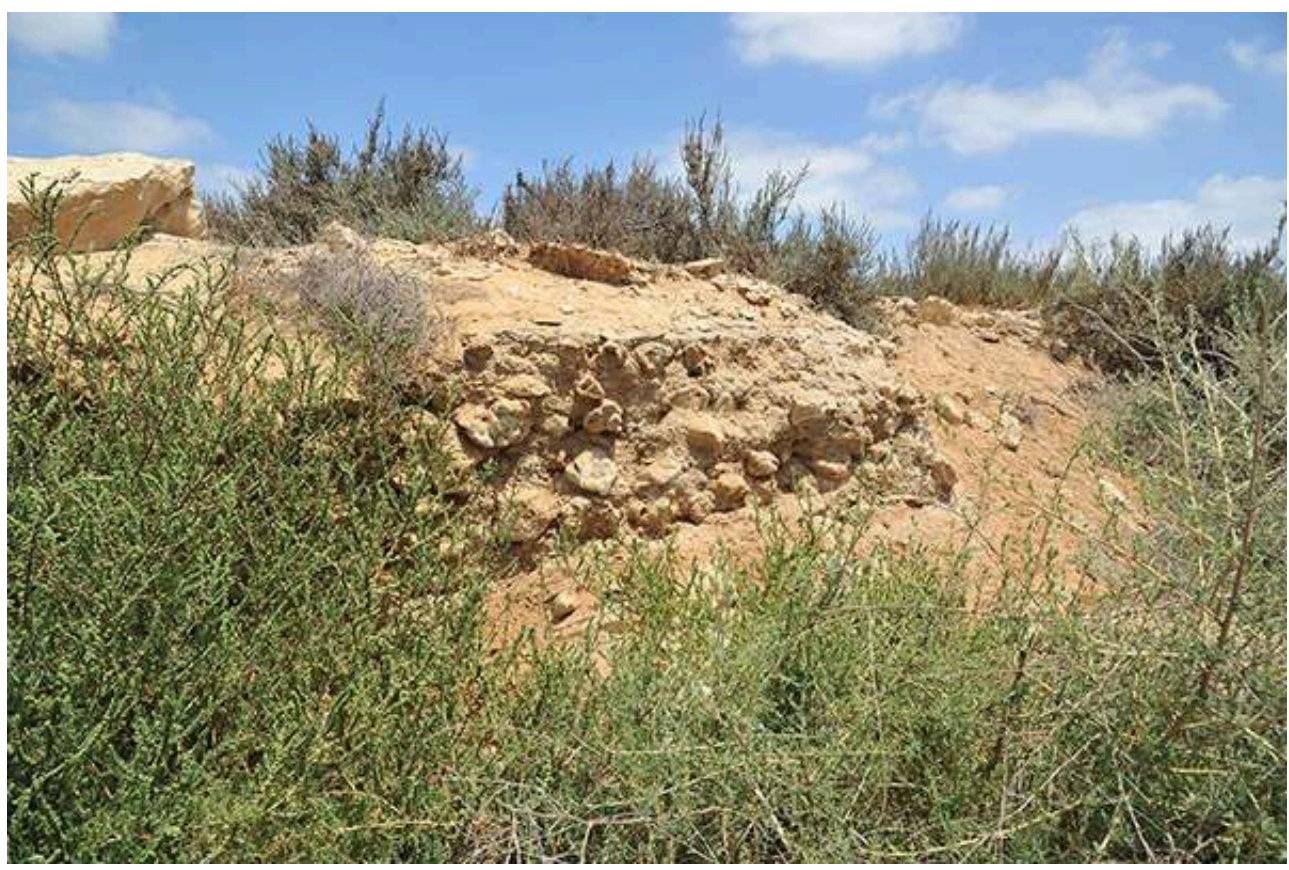

(c) Archives CEAlex. CEAlex_GMR_2020_NDMPF_024

92 À l'ouest du cimetière, une autre petite proéminence recouverte par la végétation est un vestige de la limite sud-ouest de l'enceinte et a été préservée grâce à la présence du santon Hamid Gaballah Soliman Shamata (fig. 72). 
Fig. 72. Carte archéologique de la Maréotide. GMR0171, le santon Hamid Gaballah Soliman Shamata installé sur une proéminence recouverte par la végétation, vu depuis le nord-ouest (A. Simony).

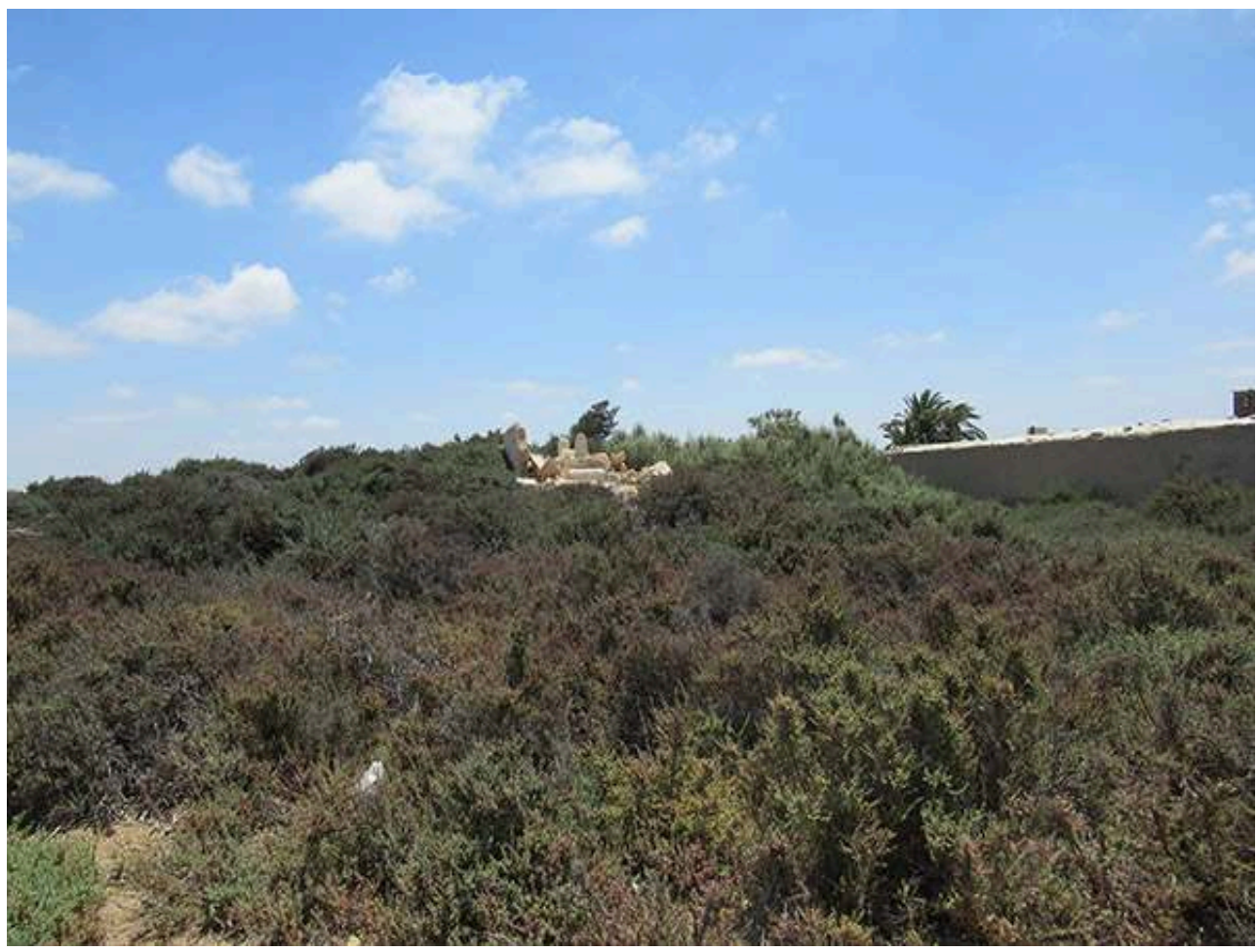

(c) Archives CEAlex. CEAlex_GMR_2020_NDMPF_025

93 À une quarantaine de mètres au nord-ouest du santon, une zone un peu surélevée présente des fragments de scories, de briques et des fragments de LRA 5/6. L'ensemble est beaucoup moins lisible qu'en 2019, le sédiment pulvérulent recouvrant la totalité du secteur.

94 L'ensemble du site livre en surface beaucoup de mobilier. Les céramiques identifiées datent l'occupation du site de l'époque romaine tardive au début de la période islamique (LRA 5/6, LRA 1, LRA 4, LR D, marmites à bord biseauté, jattes à pâte calcaire). Toutefois, la présence de quelques tessons datés du Haut-Empire (Dressel 2/4, amphore rhodienne du début du Haut-Empire, LRA 3) a été relevée cette année dans la partie sud du site.

À une cinquantaine de mètres à l'est du site une grande parcelle aplanie, transformée en terrain de football, offre en surface quelques rares fragments de céramiques remontant à la période romaine tardive et au début de la période islamique (LRA 5/6, jattes à pâte calcaire). Ce secteur correspond à l'angle nord-ouest du karm KR0327 en contact avec un second karm (KR0507) repéré sur l'image satellite Landsat 1984, et qui a aujourd'hui totalement disparu (voir fig. 65).

\subsubsection{GMR0174 - Sekeh-Hadid 46th km (Chemin de fer km 46)}

(fig. 73)

\section{Région de Naga Ahmed}


Fig. 73. Carte archéologique de la Maréotide. Croquis du site GRM0174, Sekeh-Hadid 46th km : en rose, kôms livrant les vestiges de bâtiments; en bleu, zones présentant des vestiges de murs en surface (alignements de blocs, lignes de mortier) ; en orange, carrière (V. Pichot).

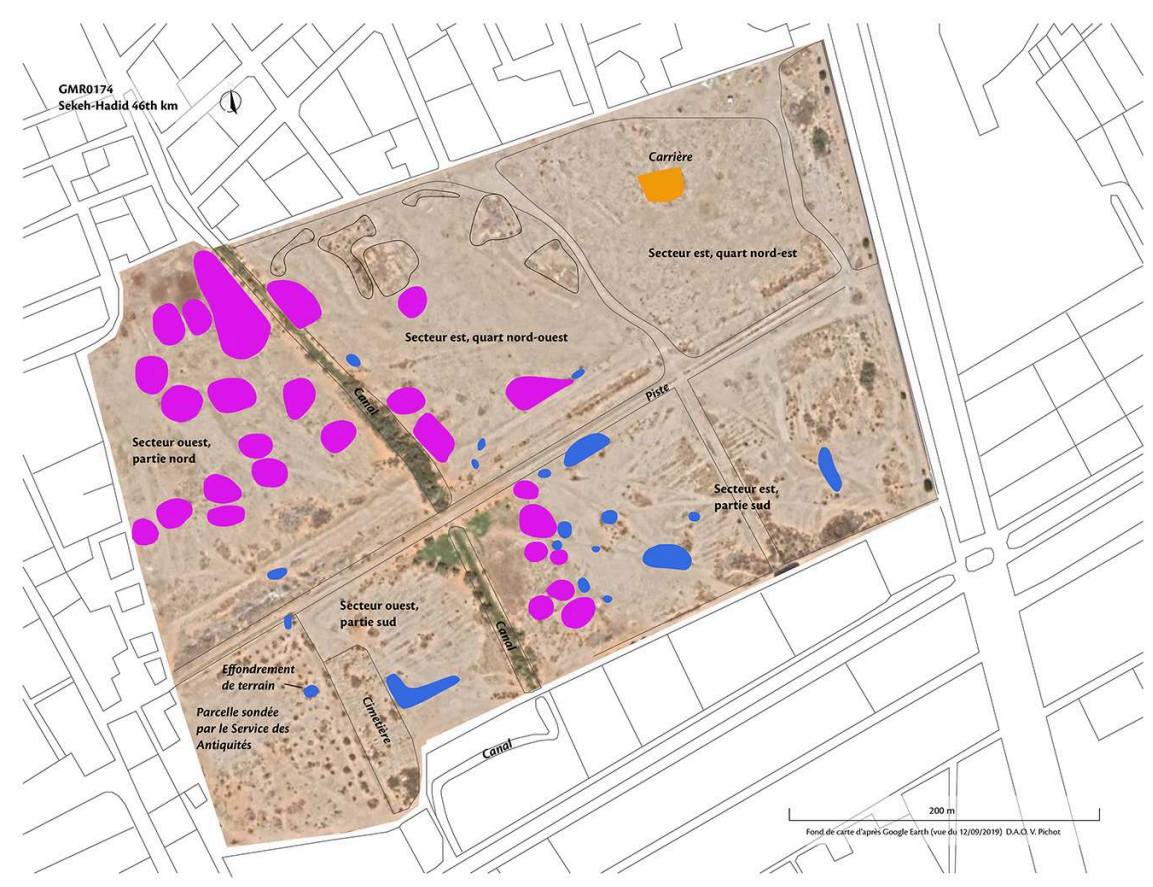

(C) Archives CEAlex. CEAlex_GMR_2020_NDMCN_009

96 La prospection de cette année dans ce secteur à proximité du village de Naga Ahmed avait pour but de compléter la documentation récoltée lors des prospections de 2019. Ce terrain de 15 ha est traversé d'est en ouest par une piste surélevée et du nord au sud par un canal qui ont défini les secteurs présentés ici.

- Secteur est, quart nord-est : carrière située sur un promontoire rocheux. Elle sert de dépotoir domestique et est presque totalement remblayée (fig. 74). Autour de la carrière, quelques fragments de céramiques sont visibles en surface. 
Fig. 74. Carte archéologique de la Maréotide. GMR0174, secteur est, quart nord-est, carrière remblayée qui sert de dépotoir domestique, vue depuis le sud-est (A. Simony).

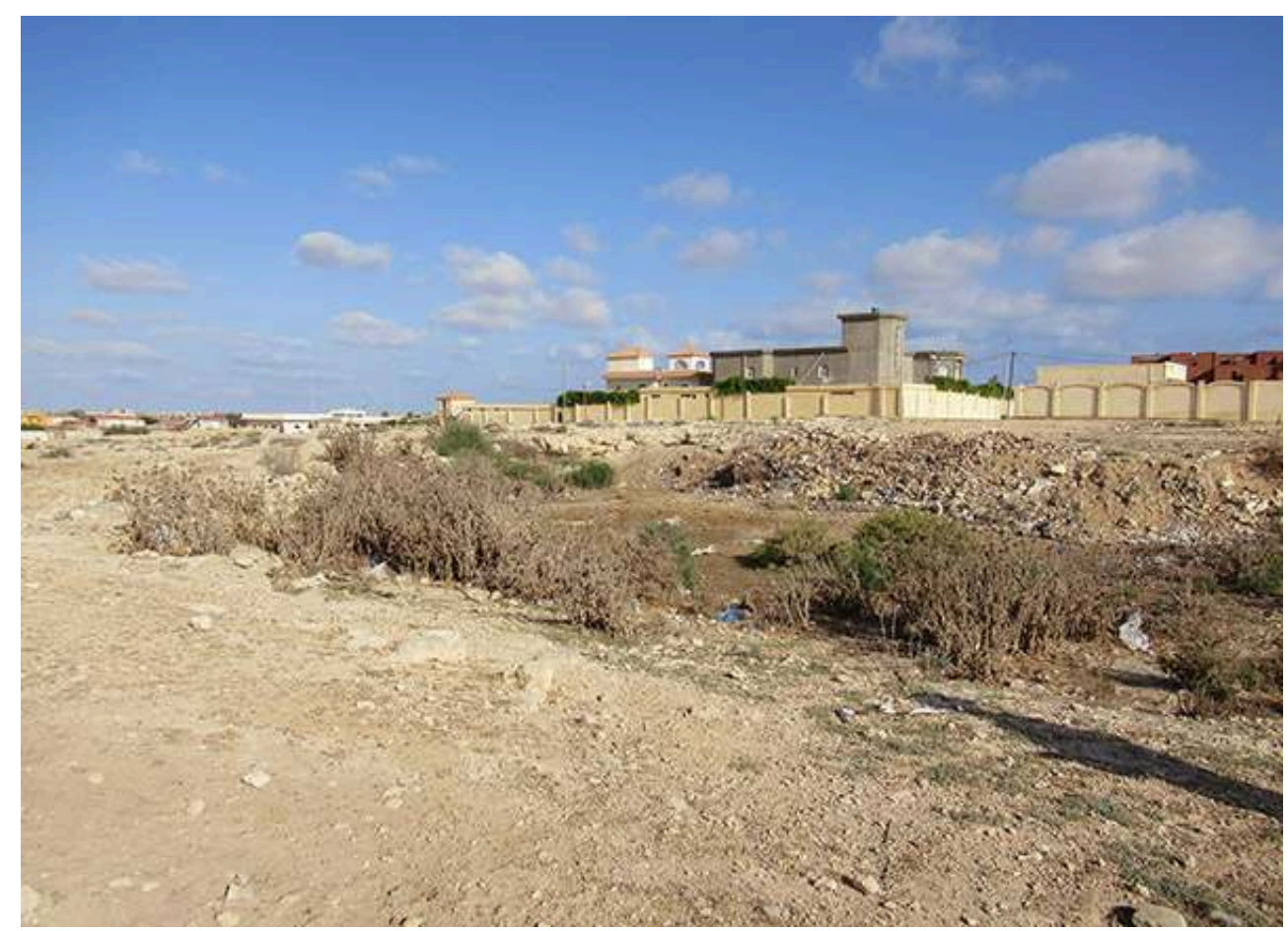

(c) Archives CEAlex. CEAlex_GMR_2020_NDMPF_026

- Secteur est, quart nord-ouest : à l'ouest de la carrière, plusieurs monticules sont visibles. Certains sont des kôms montrant des alignements de blocs et d'enduit, vestiges d'une occupation antique (fig. 75), tandis que d'autres semblent avoir été formés lors de l'arasement de certaines parties du secteur. De nombreux blocs épars et beaucoup de mobilier céramique sont visibles en surface. 
Fig. 75. Carte archéologique de la Maréotide. GMR0174, secteur est, quart nord-ouest, proéminences situées dans la partie ouest du secteur vues depuis le sud (V. Pichot).

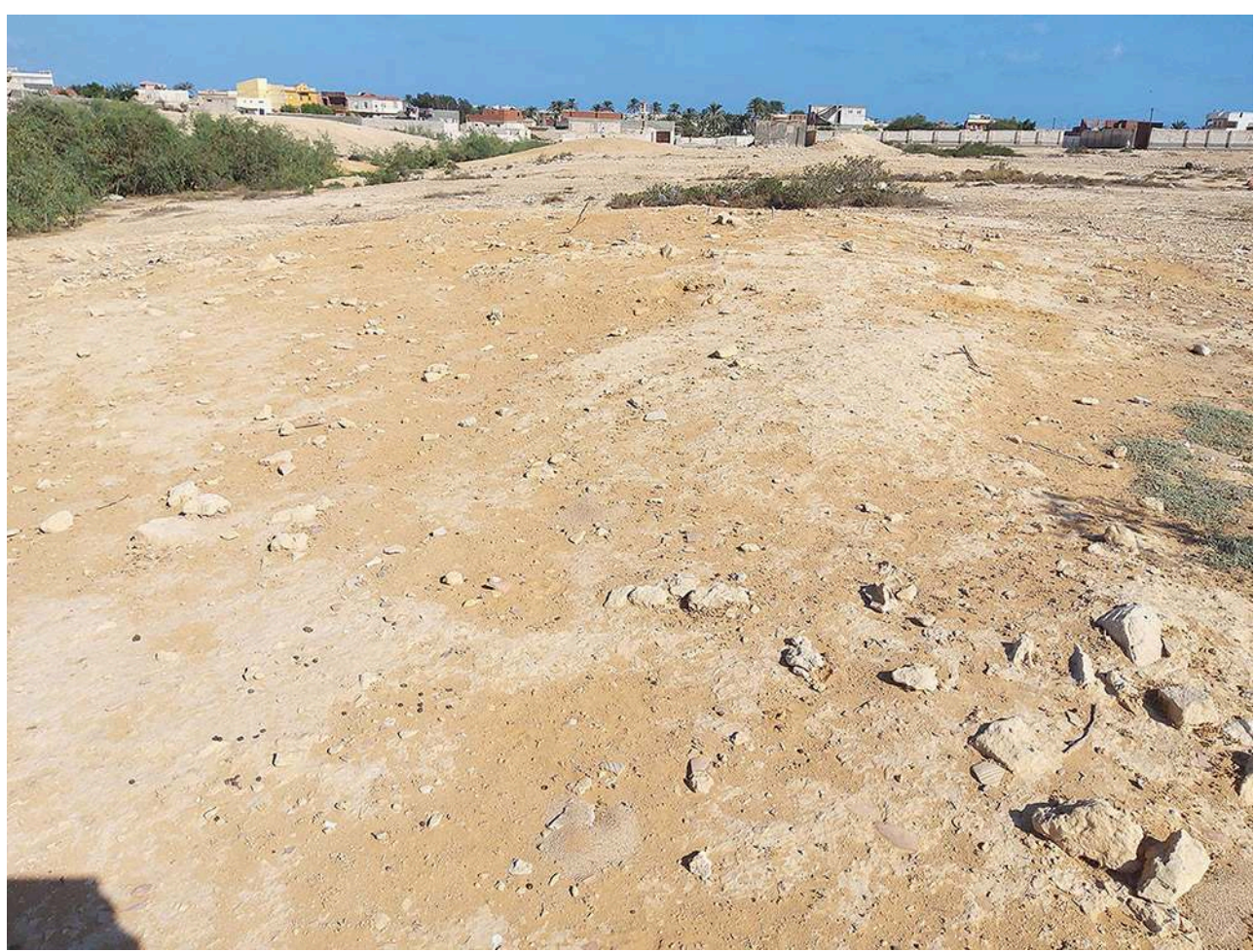

(c) Archives CEAlex. CEAlex_GMR_2020_NDMPF_027

- Secteur ouest, au nord de la piste : de nombreux fragments de céramiques sont visibles en surface sur l'ensemble du secteur. Une dizaine de kôms livrent de nombreux blocs épars, des fragments de mortier et d'enduit, quelques alignements de blocs (fig. 76), et témoignent de l'existence de plusieurs bâtiments enfouis dans ce secteur. 
Fig. 76. Carte archéologique de la Maréotide. GMR0174, secteur ouest, au nord de la piste, un des kôms vu depuis le nord-est (V. Pichot).

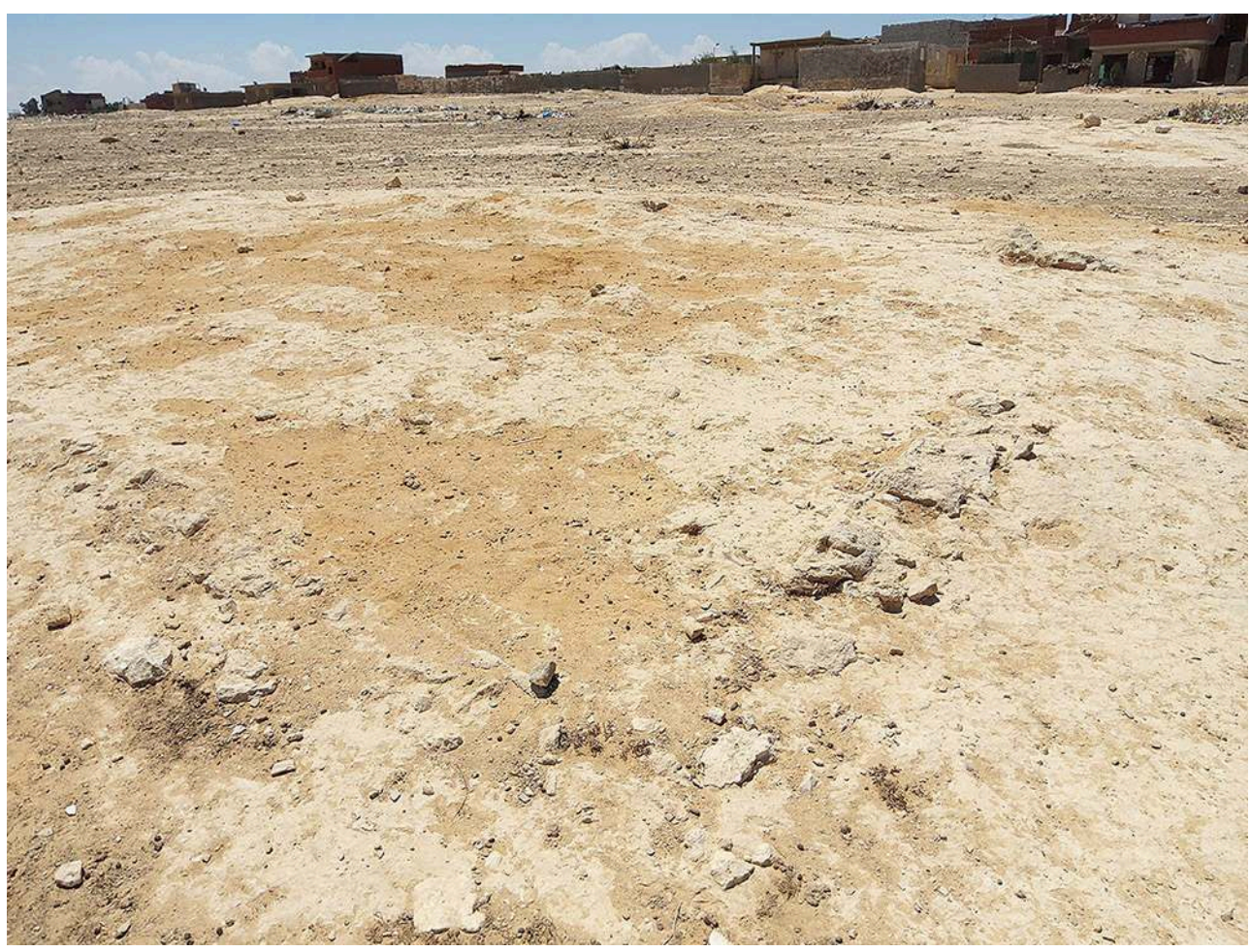

(c) Archives CEAlex. CEAlex_GMR_2020_NDMPF_028

- Secteur ouest, au sud de la piste : la partie centrale de ce secteur est occupée par un cimetière. La partie occidentale a fait l'objet de sondages de la part du MoTA; peu profonds et servant de dépotoir, ils ne livrent aucune information. De très nombreux blocs épars sont présents sur l'ensemble du secteur. Dans un petit effondrement de terrain, situé dans la partie ouest à proximité du cimetière, on note la présence d'un mur en gros blocs de calcaire local visible sur quatre assises (fig. 77), et à proximité, en surface, un alignement de blocs et mortier qui semble amorcer une courbe. À l'est du secteur quelques alignements de blocs et d'enduit sont visibles en surface (fig. 78) attestant la présence de plusieurs bâtiments. De nombreux blocs épars et de fragments de céramiques couvrent le secteur. 
Fig. 77. Carte archéologique de la Maréotide. GMR0174, secteur ouest, au sud de la piste, effondrement de terrain laissant apparaître un mur sur quatre assises, vu depuis l'ouest (V. Pichot).

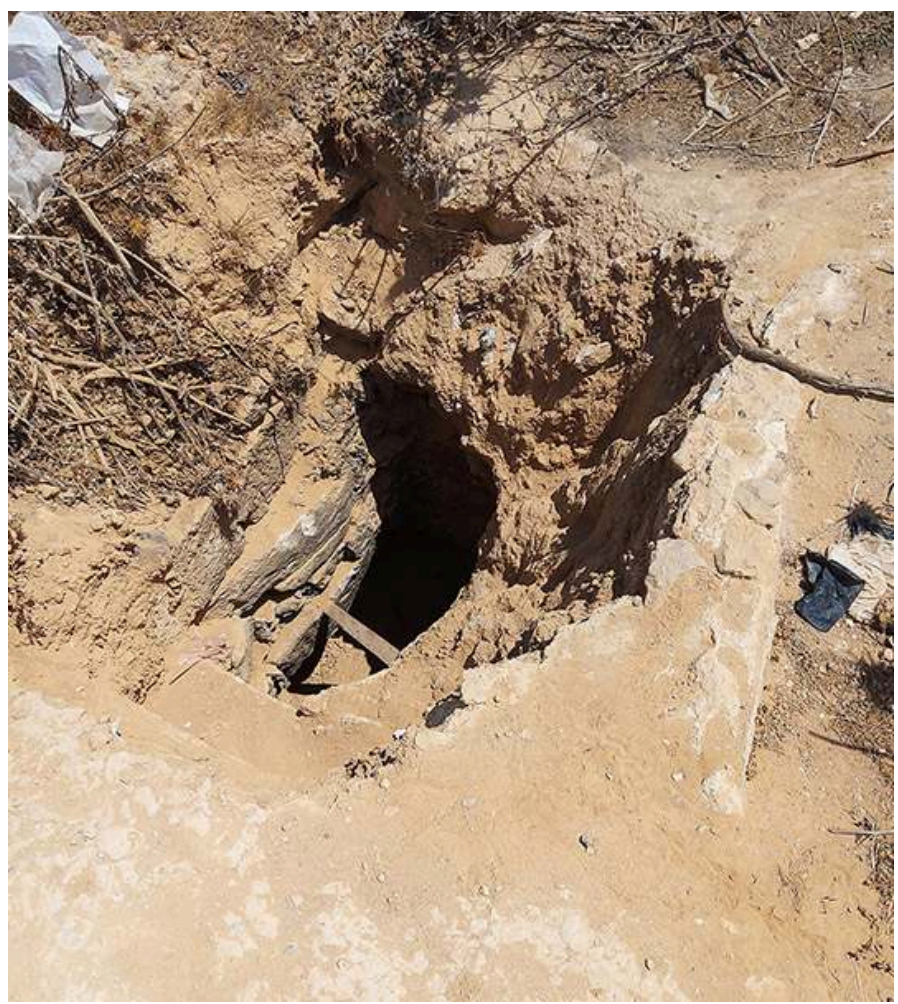

(c) Archives CEAlex. CEAlex_GMR_2020_NDMPF_029 
Fig. 78. Carte archéologique de la Maréotide. GMR0174, secteur ouest, au sud de la piste, à l'est du cimetière, alignement de blocs et d'enduit, vu depuis l'ouest (V. Pichot).

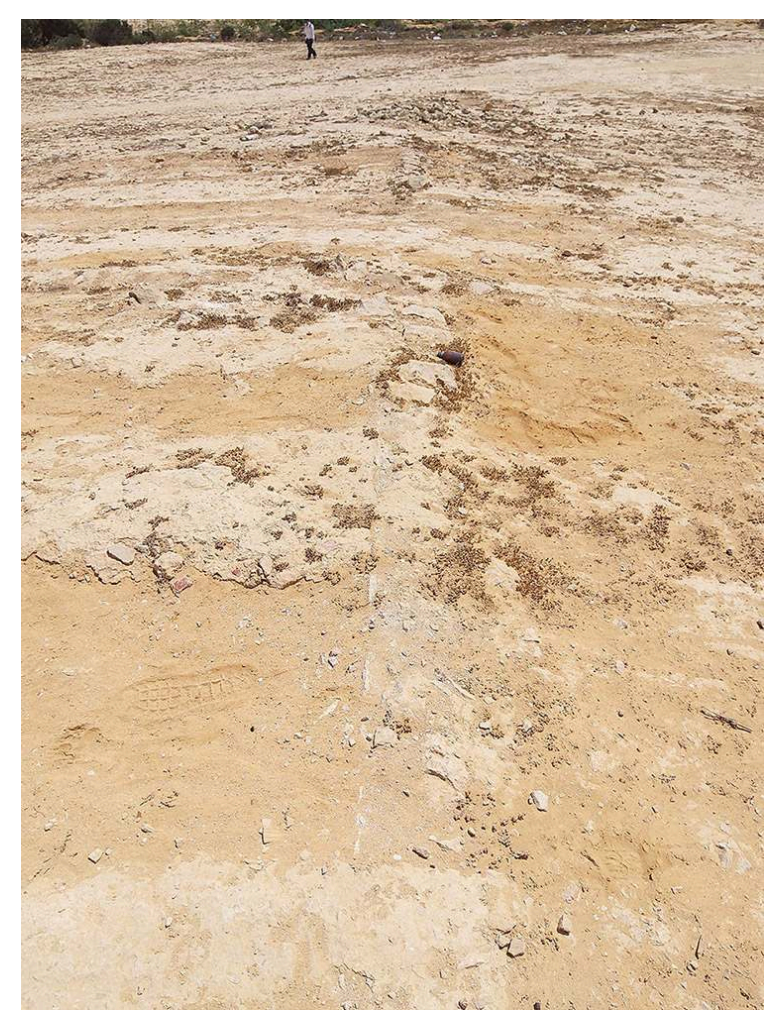

(c) Archives CEAlex. CEAlex_GMR_2020_NDMPF_030

101 - Secteur est, partie sud : l'ensemble du secteur offre de nombreux blocs épars, des fragments de mortier, d'enduit et de céramique. À proximité de la piste, on note quelques alignements de blocs et de lignes d'enduit visibles en surface. Dans la partie ouest du secteur plusieurs monticules indiquent la présence de petits bâtiments dont les élévations en briques crues se sont effondrées : murs apparents, lignes d'enduit ou de mortier visibles en surface (fig. 79). Les zones orientale et centrale du secteur ont été plus profondément remaniées (fig. 80), en partie arasées et nivelées. On remarque néanmoins plusieurs murs apparents dans la zone centrale (fig. 81), qui marquent la présence d'au moins deux bâtiments. En outre, on note des fragments de pots de sakieh dans la partie est du secteur ainsi que quelques alignements de blocs visibles en surface. 
Fig. 79. Carte archéologique de la Maréotide. GMR0174, secteur est, partie sud, vue d'un des monticules, présence de blocs et d'alignements d'enduit, vu depuis le sud-ouest (V. Pichot).

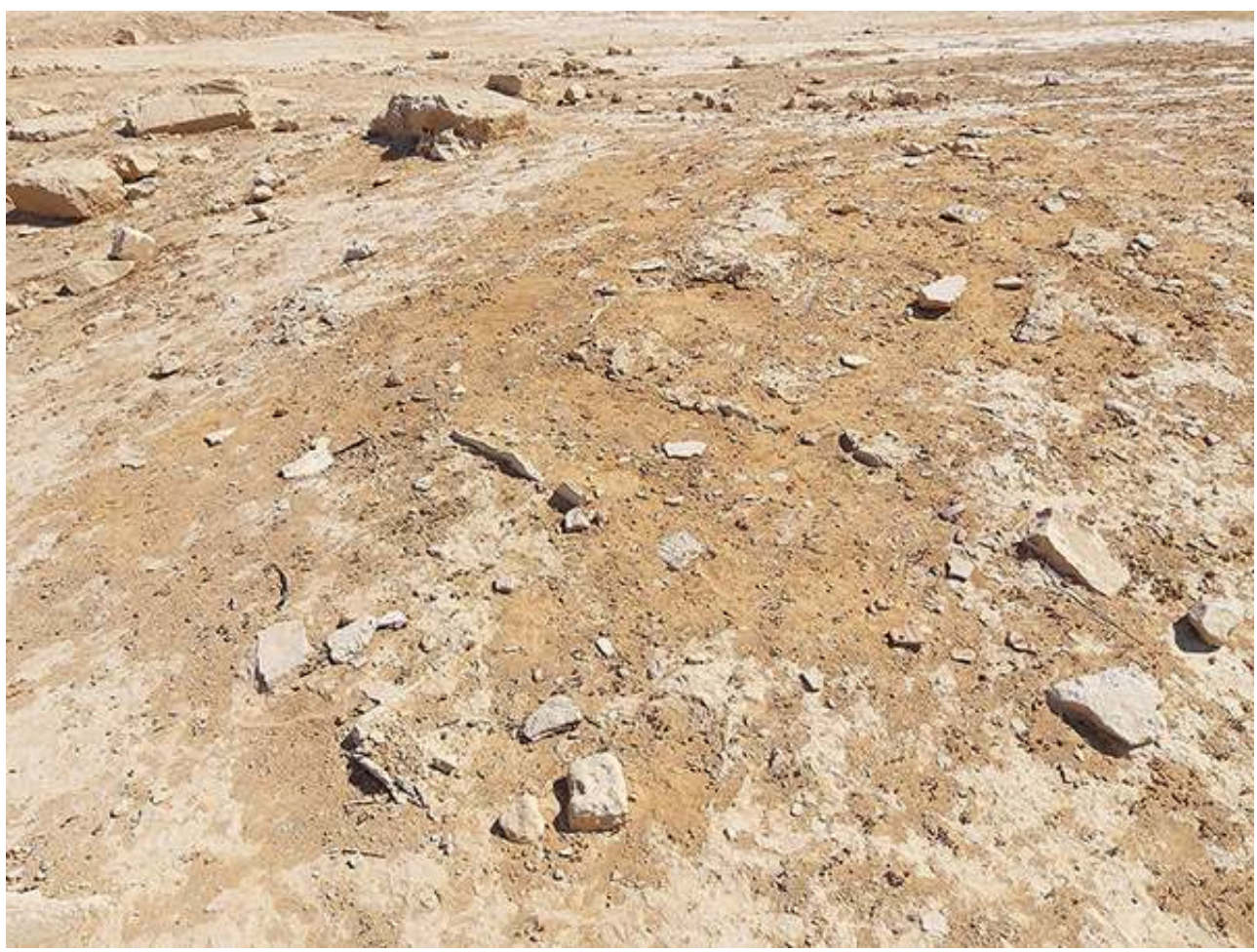

(c) Archives CEAlex. CEAlex_GMR_2020_NDMPF_031

Fig. 80. Carte archéologique de la Maréotide. GMR0174, secteur est, partie sud, zones centrale et orientale remaniées, vue partielle depuis le sud-est (A. Simony).

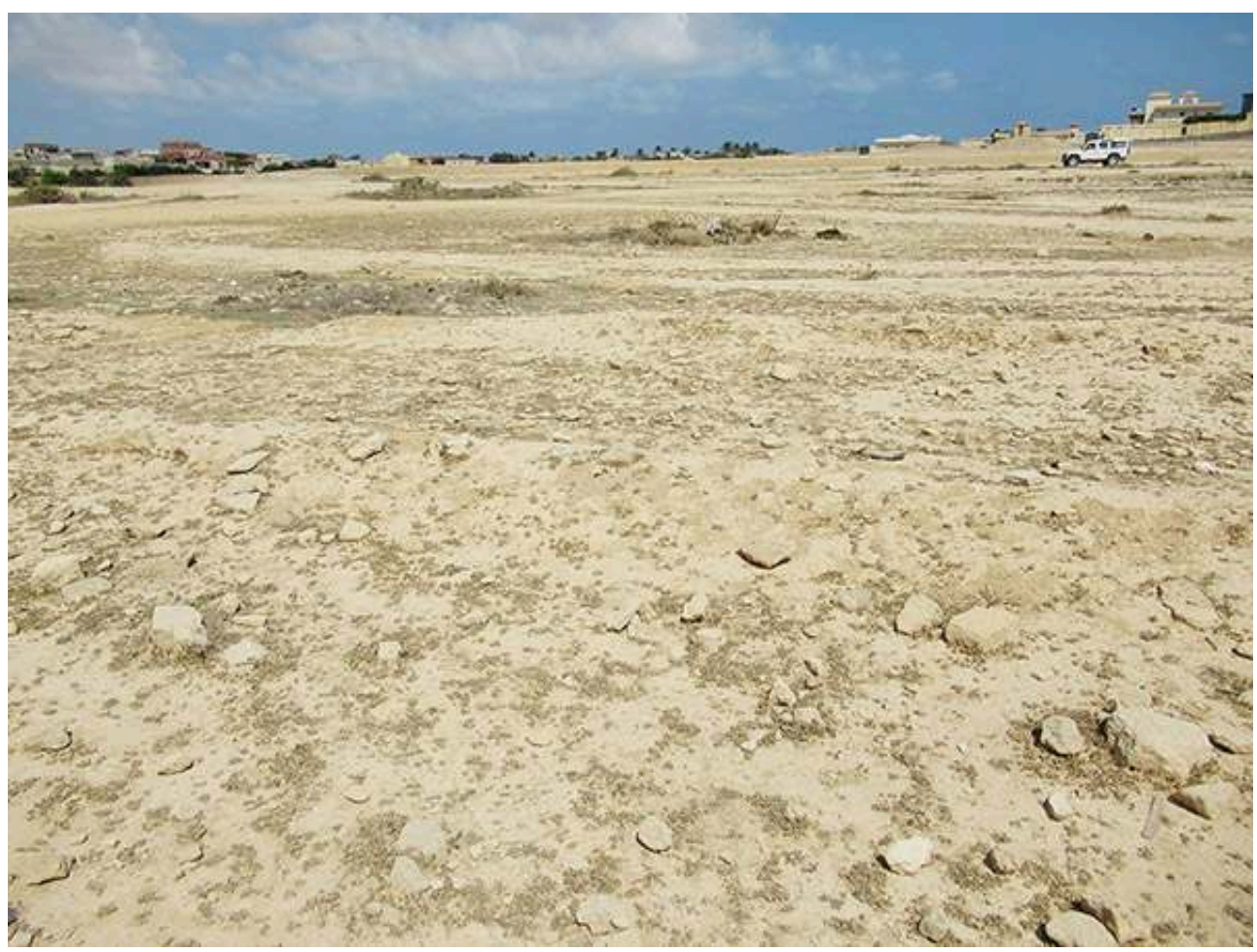

(C) Archives CEAlex. CEAlex_GMR_2020_NDMPF_032 
Fig. 81. Carte archéologique de la Maréotide. GMR0174, secteur est, partie sud, zone centrale, alignements de blocs vus depuis l'est (V. Pichot).

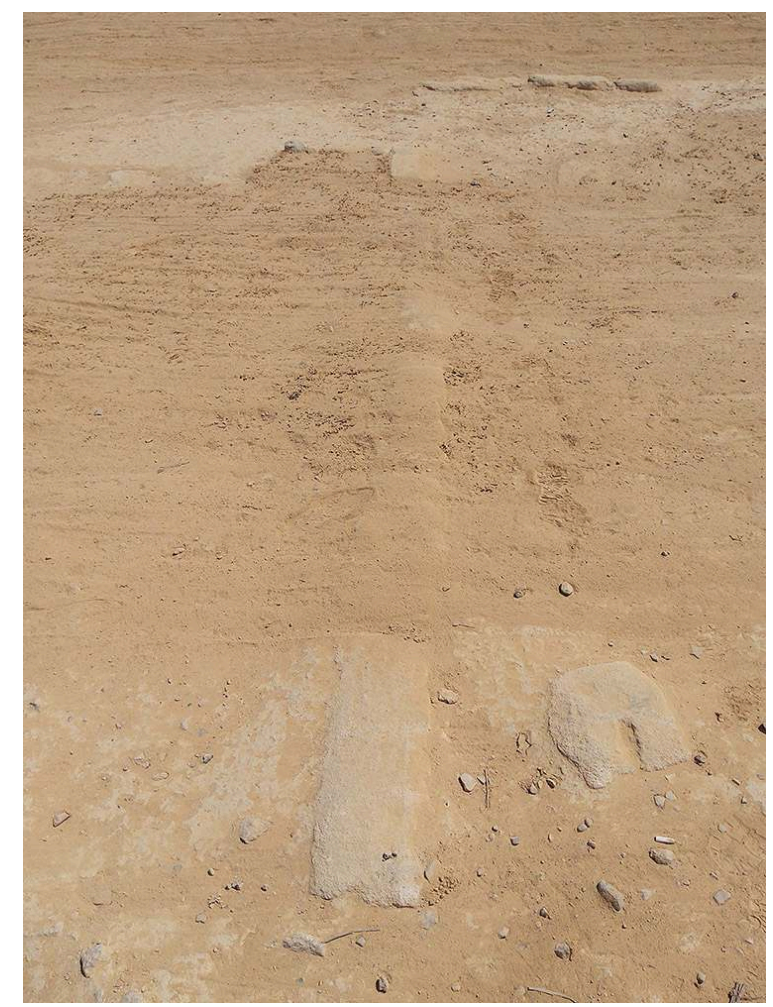

(C) Archives CEAlex. CEAlex_GMR_2020_NDMPF_033

Le mobilier céramique, peu abondant dans l'ensemble, mais présent sous forme de concentrations ponctuelles, montre une occupation continue du site de l'époque hellénistique ancienne à la période romaine tardive/début de la période islamique : anse timbrée cnidienne portant un timbre à la proue de Pasikrates, atelier de Reşadiye (fin IV ${ }^{\mathrm{e}}$-début III $\mathrm{e}$. av. J.-C.) notée en 2019, AE 2, amphore de Cos, amphore rhodienne (période hellénistique), $\mathrm{AE} 4, E S \mathrm{~A}$, jattes à marli (Haut-Empire), LR D, LRA 1 et LRA 5/6, $L R A 4$, pots de sakieh et jattes à pâte calcaire, $A R S W$, céramiques du Groupe $\mathrm{K}$ (période romaine tardive/début de la période islamique).

\subsubsection{GMR0175 - Sidi 'Abdallah el-Shayet}

\section{Région de Borg el-Arab}

Situé à ca $2 \mathrm{~km}$ au sud-ouest du GMR0174 entre la ride IV et la ride V une anomalie de terrain repérée sur les images satellites Google Earth correspond aux restes d'un karm (KR0045) topographié en partie sur la carte du survey de $1940^{24}$.

Le site est recouvert par un sédiment argileux jaune-orange et se compose de trois grandes proéminences, séparées par des zones arasées et nivelées, et livrant en surface de nombreux blocs épars, qui correspondent à l'angle nord-est de l'enceinte du karm. Les proéminences sud-ouest et orientale (fig. 82) offrent de nombreux tessons en surface et des petits blocs épars surtout sur leurs pentes. Aucune structure apparente n'a été détectée sur ces deux secteurs. Un santon (Sheikh 'Abdallah el-Shayet) associé à un cimetière occupe la partie ouest de la proéminence orientale (fig. 83). 
Fig. 82. Carte archéologique de la Maréotide. GMR0175, au second plan à gauche la proéminence sud-ouest, à l'arrière-plan à droite la proéminence orientale, vues depuis le sud-ouest (V. Pichot).

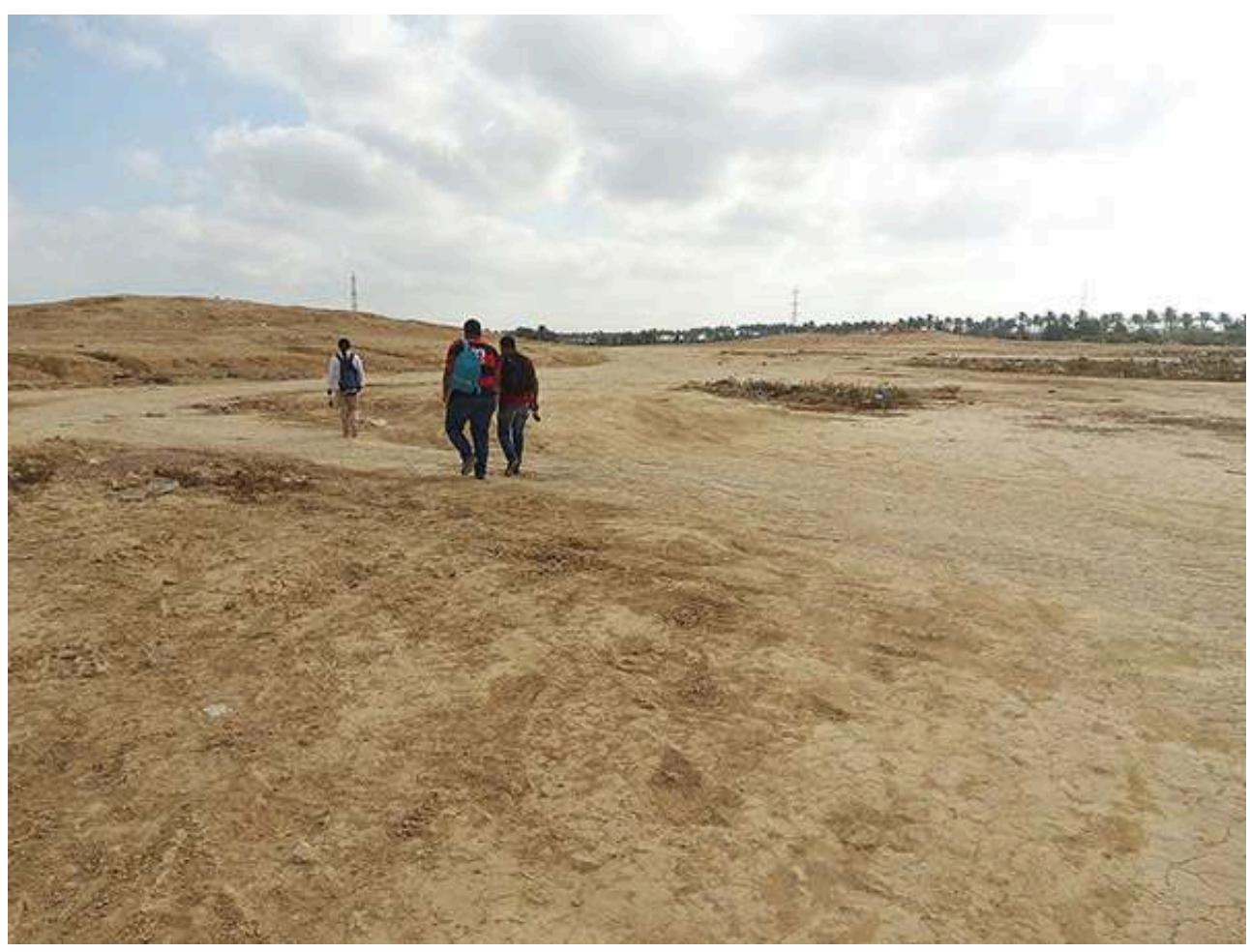

(c) Archives CEAlex. CEAlex_GMR_2020_NDMPF_034

Fig. 83. Carte archéologique de la Maréotide. GMR0175, proéminence orientale avec le santon Sheikh 'Abdallah el-Shayet et une partie du cimetière associé, vue depuis le nord-est (V. Pichot).

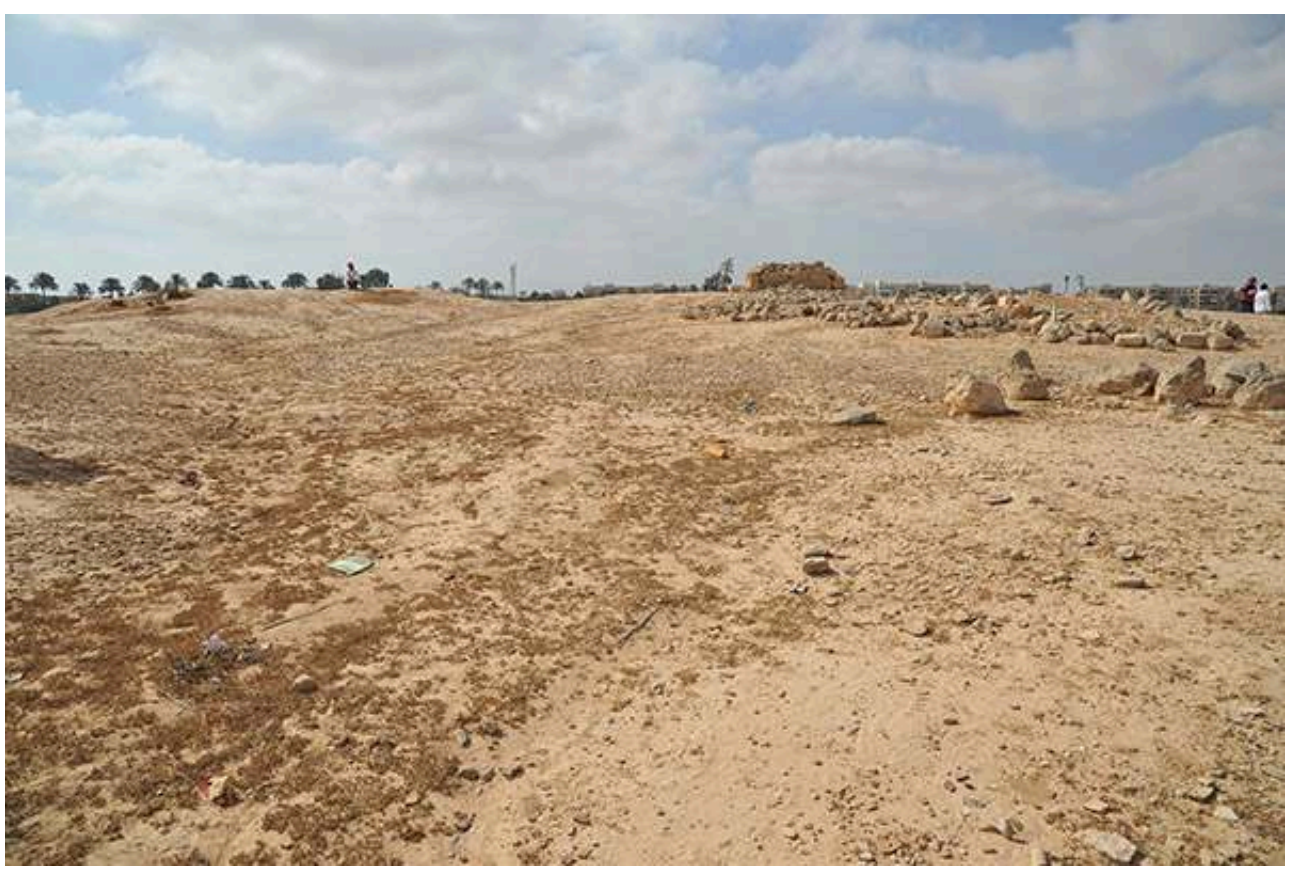

(c) Archives CEAlex. CEAlex_GMR_2020_NDMPF_035 
106 Quelques vestiges sont visibles à la surface de la proéminence nord. Au sud-ouest on détecte une installation vinicole (fig. 84) : alignements de pierres et de mortier (fouloir, cuve). Au nord, de nombreux blocs épars et quelques murs en surface (fig. 85) témoignent de plusieurs ensembles de bâtiments allongés (fonction de stockage). Ces structures sont associées à un matériel de surface daté des périodes romaine et romaine tardive. Toutefois, la présence d'amphores de type AE 4, datées du HautEmpire, est particulièrement significative dans ce secteur.

Fig. 84. Carte archéologique de la Maréotide. GMR0175, proéminence au nord du site, vue partielle de l'installation vinicole avec ses alignements d'enduits, vue depuis le sud-est, à l'arrière-plan la proéminence sud-ouest (V. Pichot).

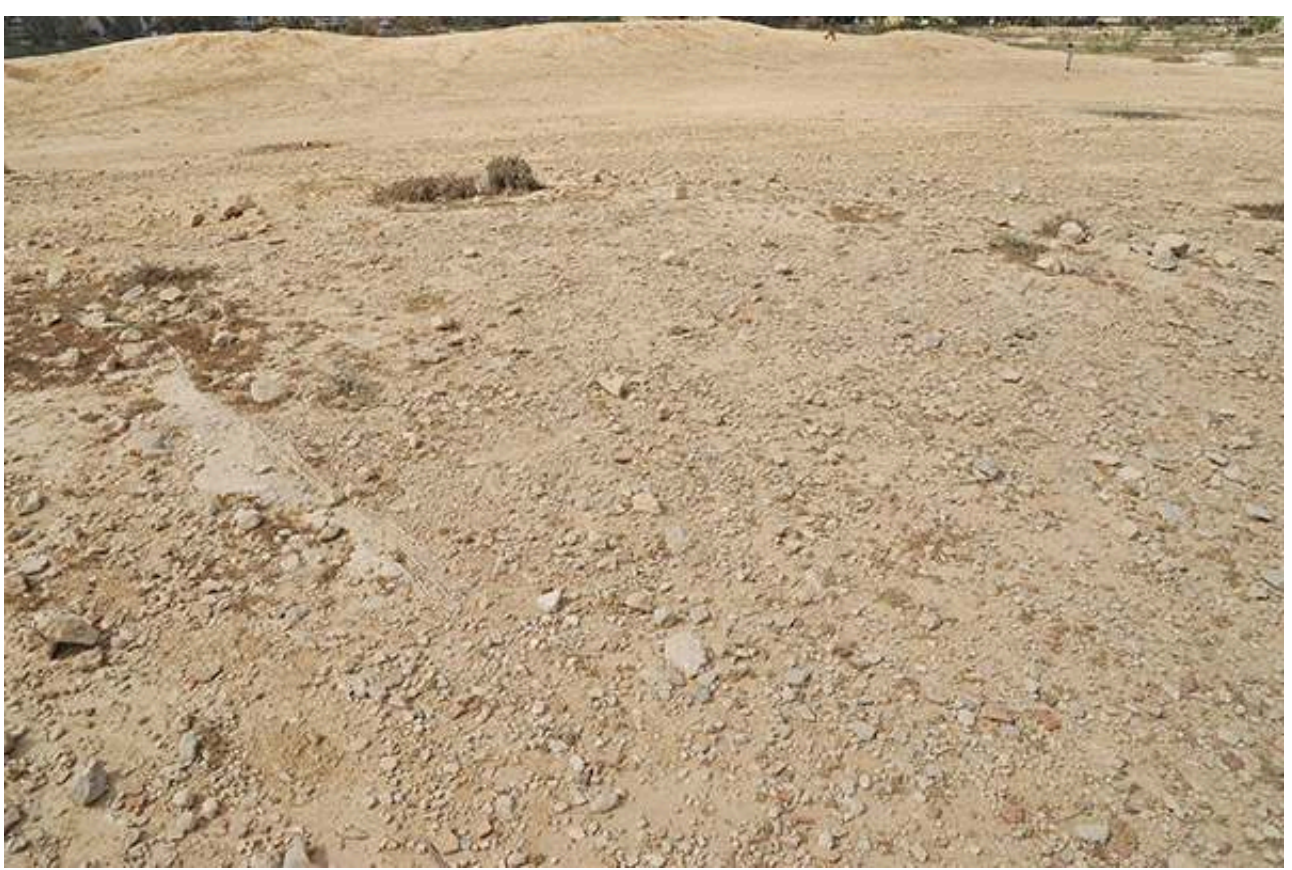

(C) Archives CEAlex. CEAlex_GMR_2020_NDMPF_036 
Fig. 85. Carte archéologique de la Maréotide. GMR0175, proéminence au nord du site, blocs épars et alignements de blocs, vus depuis le sud (V. Pichot).

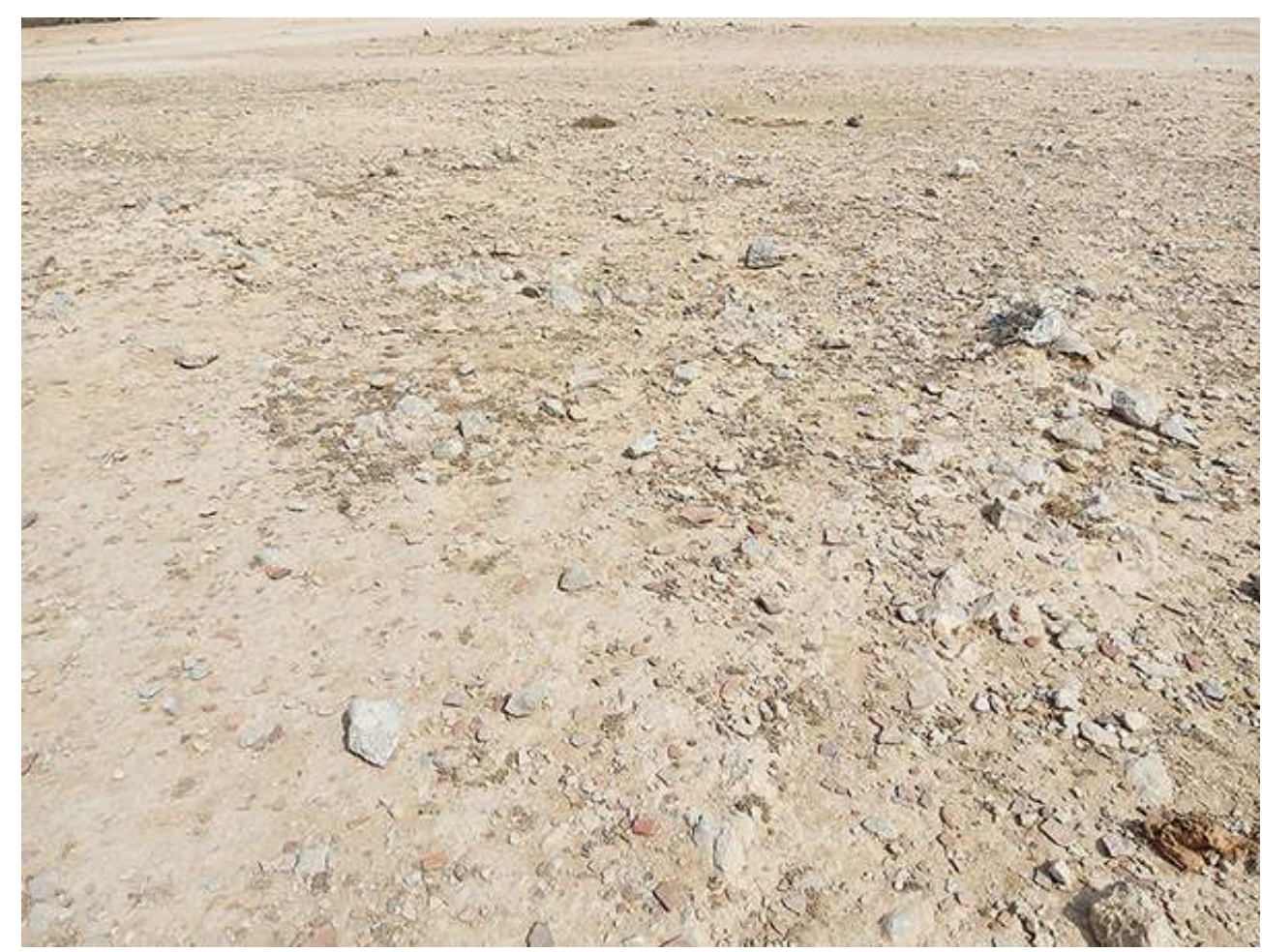

(C) Archives CEAlex. CEAlex_GMR_2020_NDMPF_037

L'ensemble du mobilier suggère que l'occupation du site couvre la période ptolémaïque (amphore rhodienne, amphore cnidienne, $\mathrm{AE} 2)$, le Haut-Empire $(\mathrm{AE} 3, \mathrm{AE} 4$, jattes à marli, marmites, cratère égyptien d'imitation chypriote, jatte à marli) et de l'époque romaine tardive/début de la période islamique (LRA 1, LRD, marmite cilicienne, LRA 5/6).

\subsection{Présentation de sites prospectés : le secteur de Gharbaneyat}

\subsubsection{GMR0158 - Karm el-Qatf}

(fig. 86)

\section{Région d'el-Gharbaneyat}


Fig. 86. Carte archéologique de la Maréotide. Croquis du site GMR0158, Karm el-Qatf : en bleu, limites actuelles du site et secteurs définis; en orange, anomalies (karm) topographiées en 1914 ; en tireté vert, restitution du karm (V. Pichot).

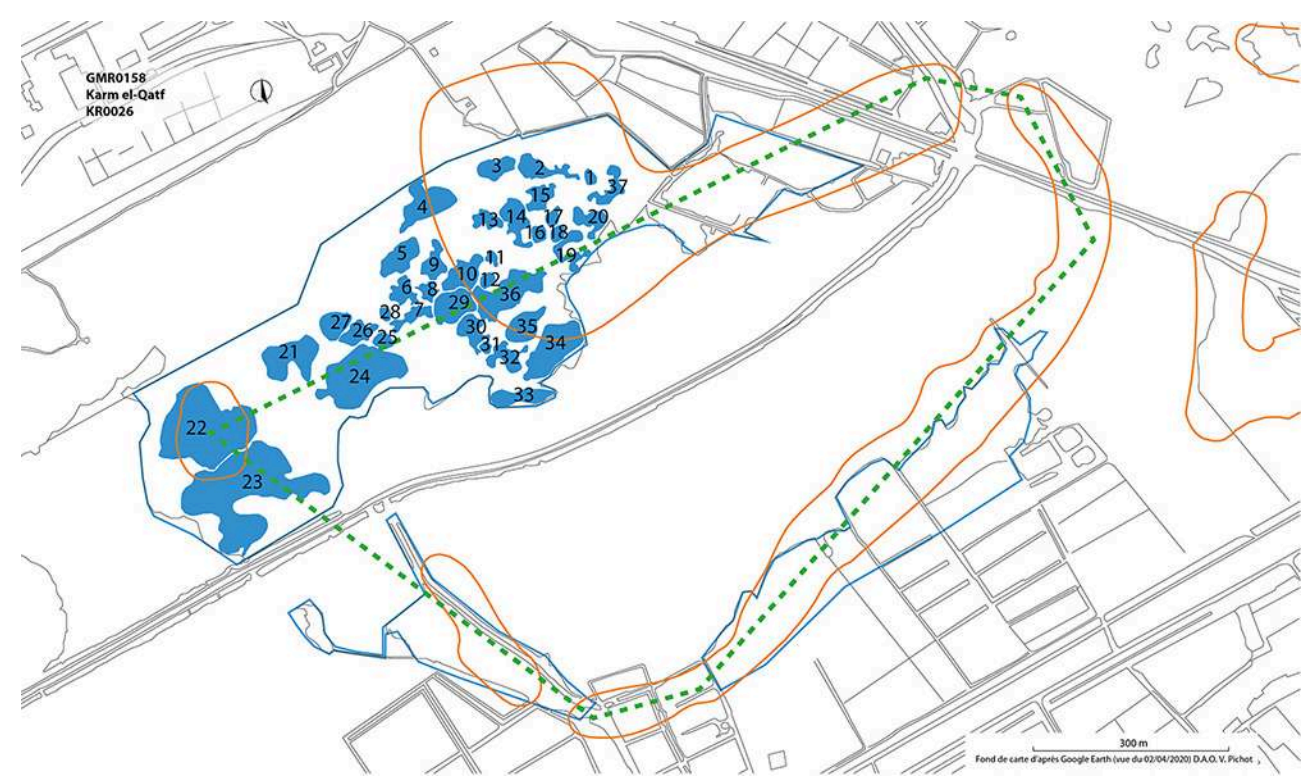

(c) Archives CEAlex. CEAlex_GMR_2020_NDMCN_010

À environ 5 km à l'ouest de Borg el-Arab, le site de Karm el-Qatf se situe directement au sud de la grande cimenterie "Amreyah Cement Co» installée sur la ride III et ses pentes jusqu'au lac et dont l'emplacement est mentionné sur les cartes anciennes comme « Gebel el-Hammâm ». Il s'agit d'un grand karm (KR0026) d'1,45 km est-ouest et de 1,13 km nord-sud, topographié sur les cartes de l'Atlas Lower Egypt 1914 et du survey de 1940 et dont il ne reste que quelques portions d'enceinte aplanie (au sud-ouest, au sud et au nord-est) et un secteur mieux conservé au nord-ouest.

Une partie de l'enceinte sud du karm se distingue sur les images satellites Google Earth. Difficilement accessible, elle est située, comme la plus grande partie du karm, en zone marécageuse et livre peu de choses réellement lisibles. L'enceinte a été rasée, aplanie et, à certains endroits, décaissée. On en distingue néanmoins en surface les vestiges avec un sédiment argileux pulvérulent jaune-orange caractéristique.

110 Un vaste ensemble, partiellement exploré en 2019, se développe en partie sur l'enceinte nord du karm au niveau de son angle nord-ouest, sur lequel les vestiges de structures antiques abondent. L'ensemble des 37 secteurs accessibles, bordé au nord et à l'ouest de promontoires rocheux qui appartiennent à la ride III, est couvert d'un sédiment argileux pulvérulent jaune-orange, provenant de la détérioration de l'enceinte du karm et de murs en briques crues. Certaines zones sont couvertes d'une végétation basse et dense qui rend difficile la lecture du site. On note que d'autres récemment désherbées livrent les vestiges de nombreux bâtiments anciennement fouillés (fouille clandestine ou très ancienne fouille du MoTA ?) (fig. 87). Malgré la densité des vestiges et la difficulté de lecture du site, nous pouvons néanmoins souligner quelques secteurs remarquables. 
Fig. 87. Carte archéologique de la Maréotide. GMR0158, secteur 31, bâtiment anciennement fouillé, vu depuis le nord-ouest (I. Awad).

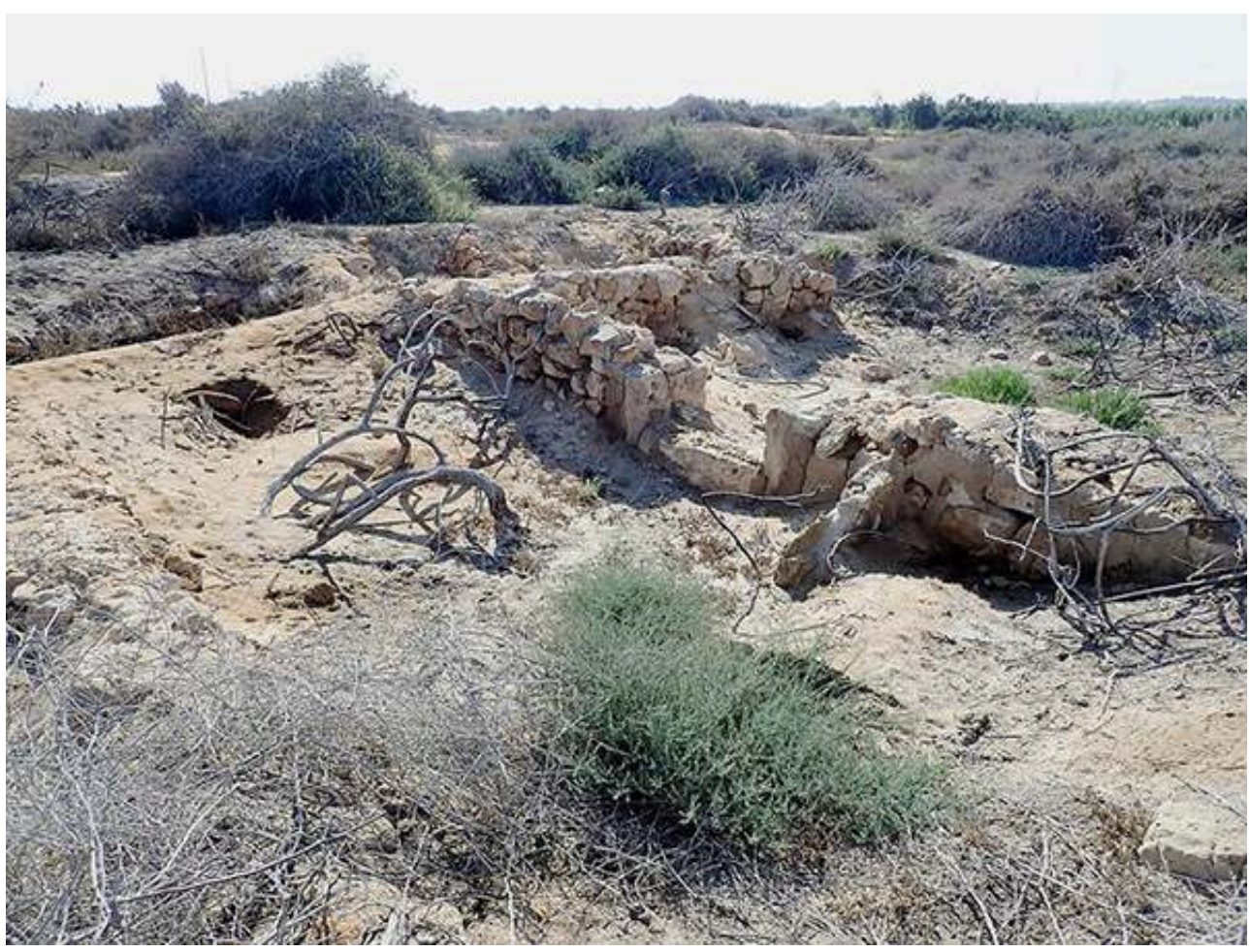

(c) Archives CEAlex. CEAlex_GMR_2020_NDMPF_038

- Un grand kôm, associé à deux kôms satellites (secteur 4, fig. 88), sur lequel quelques murs affleurent en surface, domine le site au nord. Il pourrait s'agir d'une zone d'habitat permettant aussi la surveillance du karm. 
Fig. 88. Carte archéologique de la Maréotide. GMR0158, secteur 4, au second plan un des kôm satellites, à l'arrière-plan le grand kôm, vu depuis l'ouest (I. Awad).

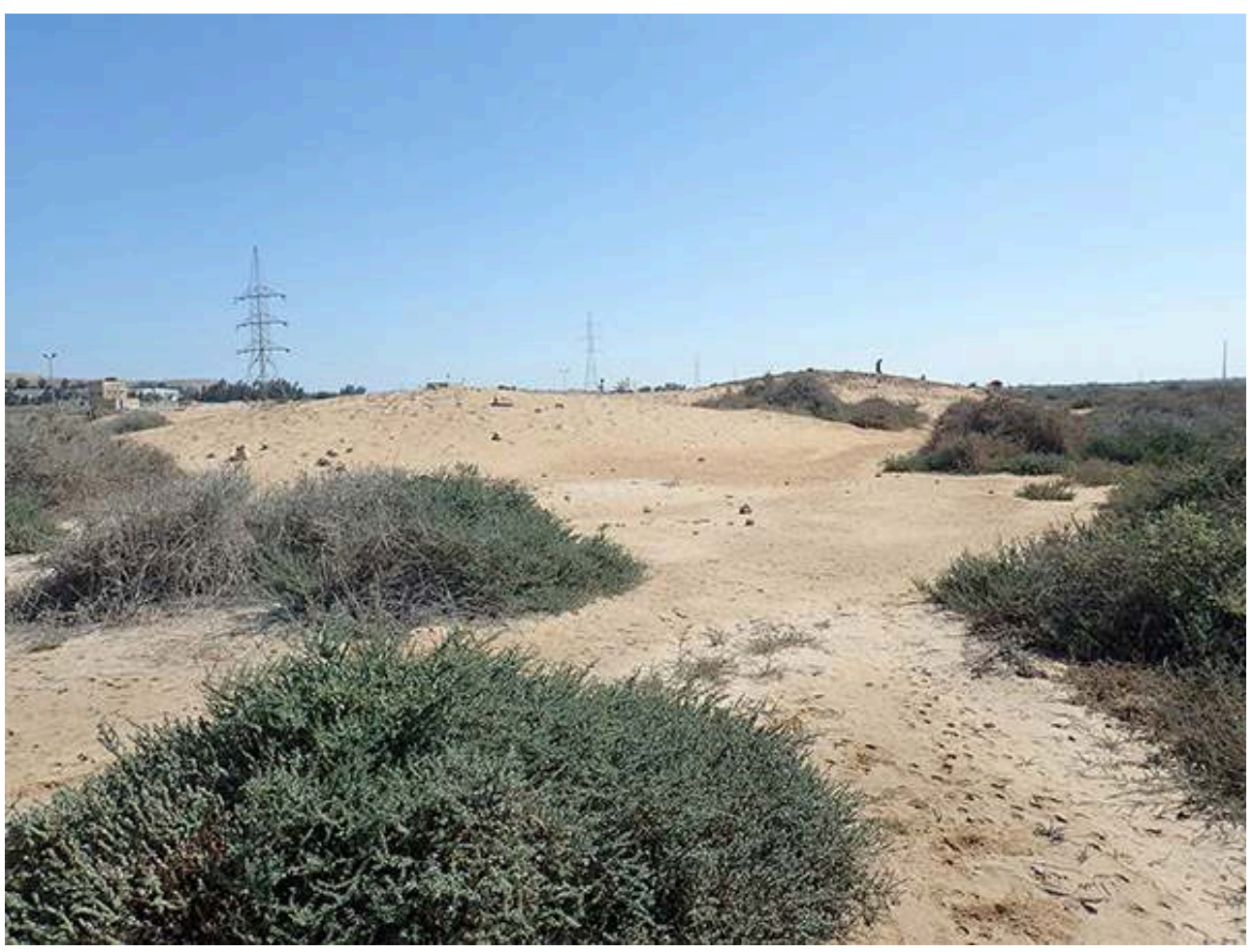

(c) Archives CEAlex. CEAlex_GMR_2020_NDMPF_039

- Le reste du site est rythmé par une succession de nombreux petits kôms (fig. 89) qui révèlent une certaine homogénéité d'aspect: sous le sédiment de surface de nombreuses lignes d'enduits, parfois associés à des murs, apparaissent, permettant d'identifier dans certains cas des canalisations, des structures circulaires ou encore de petits bâtiments. 
Fig. 89. Carte archéologique de la Maréotide. GMR0158, vue partielle du site, partie ouest, depuis le nord-est (I. Awad).

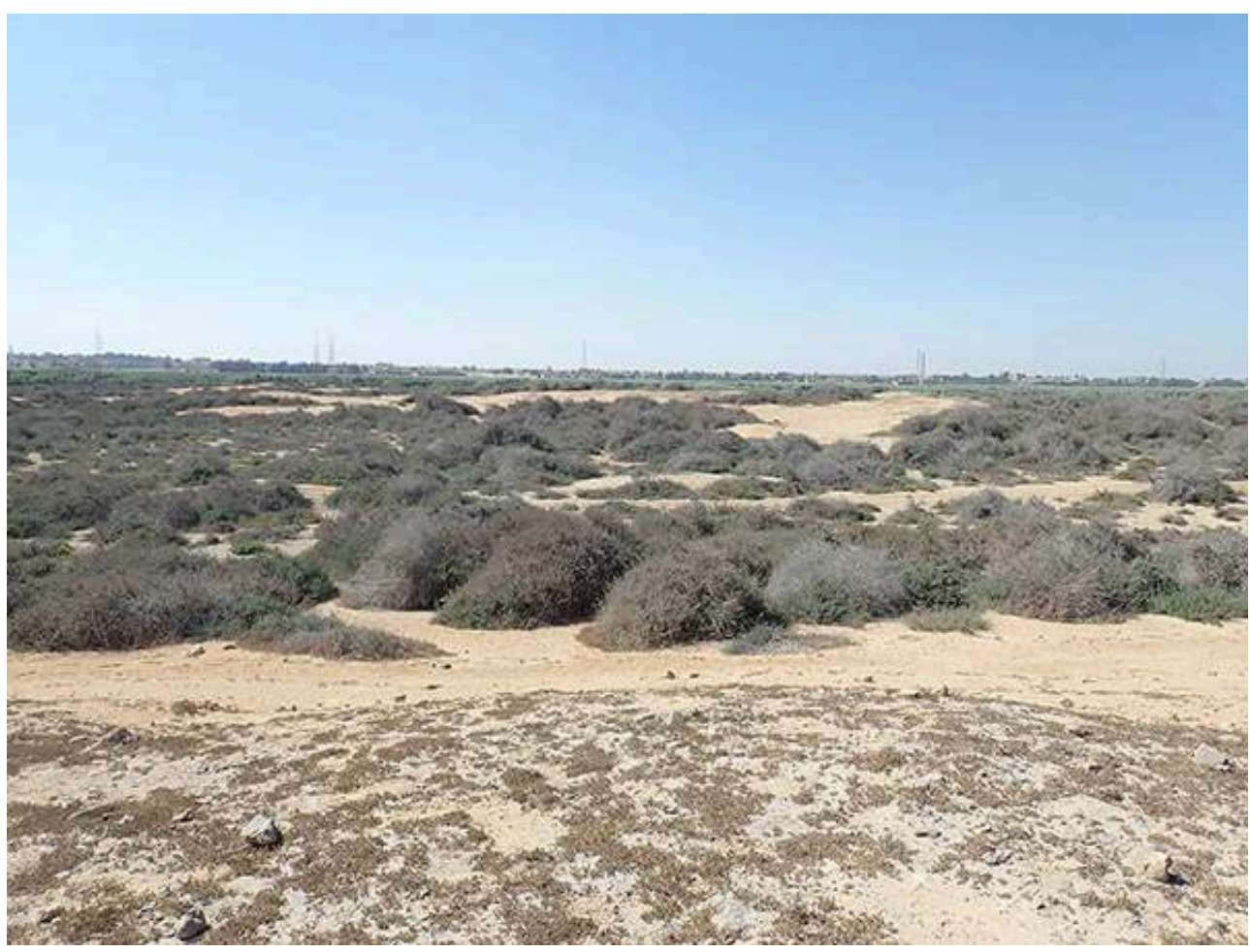

(c) Archives CEAlex. CEAlex_GMR_2020_NDMPF_040

111 Les fonctions de ces vestiges sont généralement difficiles à identifier, toutefois la destination agricole de certains bâtiments ne fait aucun doute, tandis que d'autres sont probablement à fonction domestique. Nous pouvons noter la présence :

- d'installations vinicoles, au moins cinq structures pourraient être identifiées comme telles sur les secteurs 6 (fig. 90) , 8, 12, 18 et 20 ; 
Fig. 90. Carte archéologique de la Maréotide. GMR0158, secteur 6, bâtiment visible en surface interprété comme une installation vinicole, vue depuis l'est (V. Pichot).

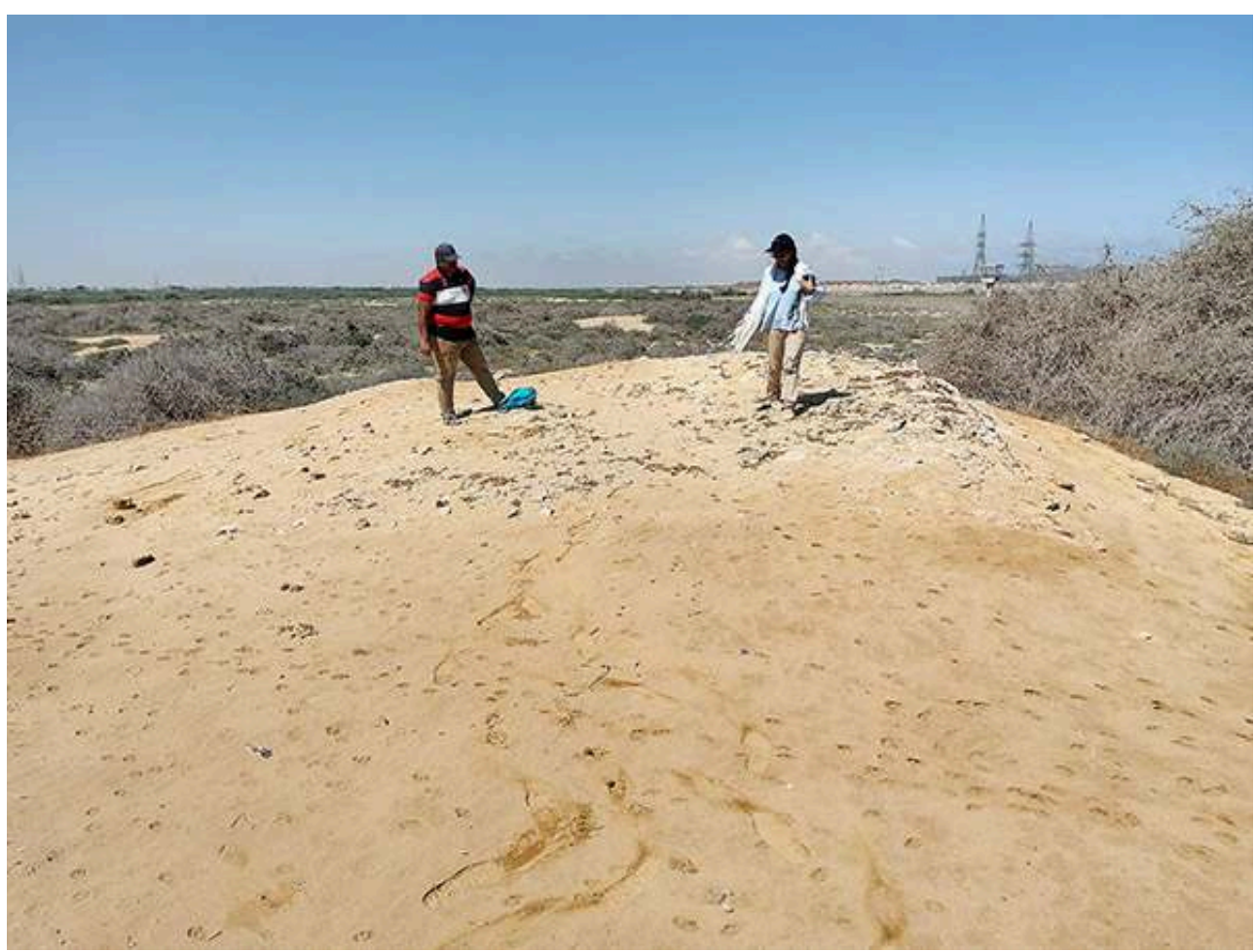

(C) Archives CEAlex. CEAlex_GMR_2020_NDMPF_041

- d'un bâtiment très allongé, situé en contrebas d'un petit kôm qui livre les vestiges d'une possible installation vinicole (?), évoquant une fonction de stockage (secteur 20) (fig. 91) ; 
Fig. 91. Carte archéologique de la Maréotide. GMR0158, secteur 20, alignement de blocs visible en surface appartenant à un bâtiment allongé (V. Pichot).

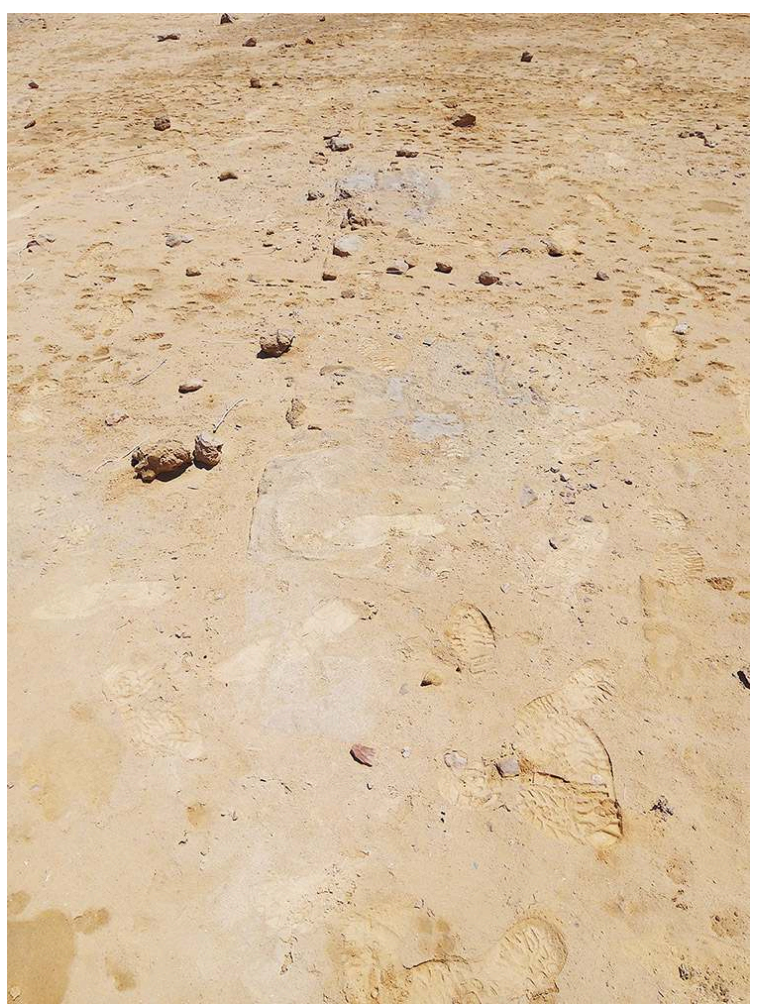

(c) Archives CEAlex. CEAlex_GMR_2020_NDMPF_042

- d'un dépotoir d'atelier de production d'amphores LRA 5/6 repérable par des tessons surcuits et déformés (fig. 92), recouvrant en partie un petit bâtiment (secteur 18) associé à un dépotoir domestique plus au nord (secteur 17) ; 
Fig. 92. Carte archéologique de la Maréotide. GMR0158, secteur 18, détail de l'atelier de production d'amphore $L R A$ 5/6 visible en surface (A. Simony).

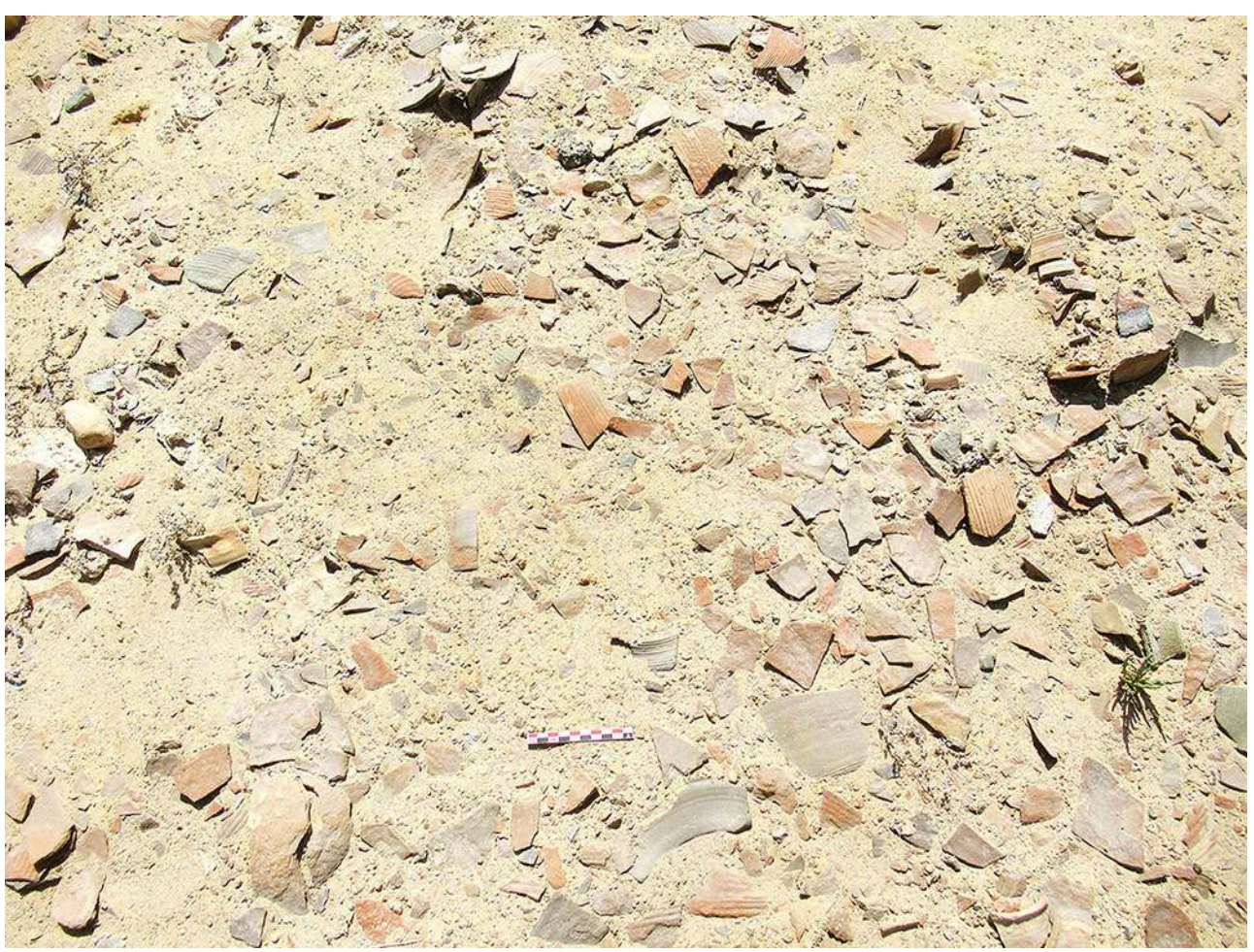

(C) Archives CEAlex. CEAlex_GMR_2020_NDMPF_043

- d'un cimetière (secteur 8 , partie nord) qui semble très ancien, et dont les tombes sont aujourd'hui difficiles à identifier (fig. 93). 
Fig. 93. Carte archéologique de la Maréotide. GMR0158, secteur 8 , au second plan à gauche cimetière ancien, à droite bâtiment interprété comme une installation vinicole, vu depuis l'ouest (V. Pichot).

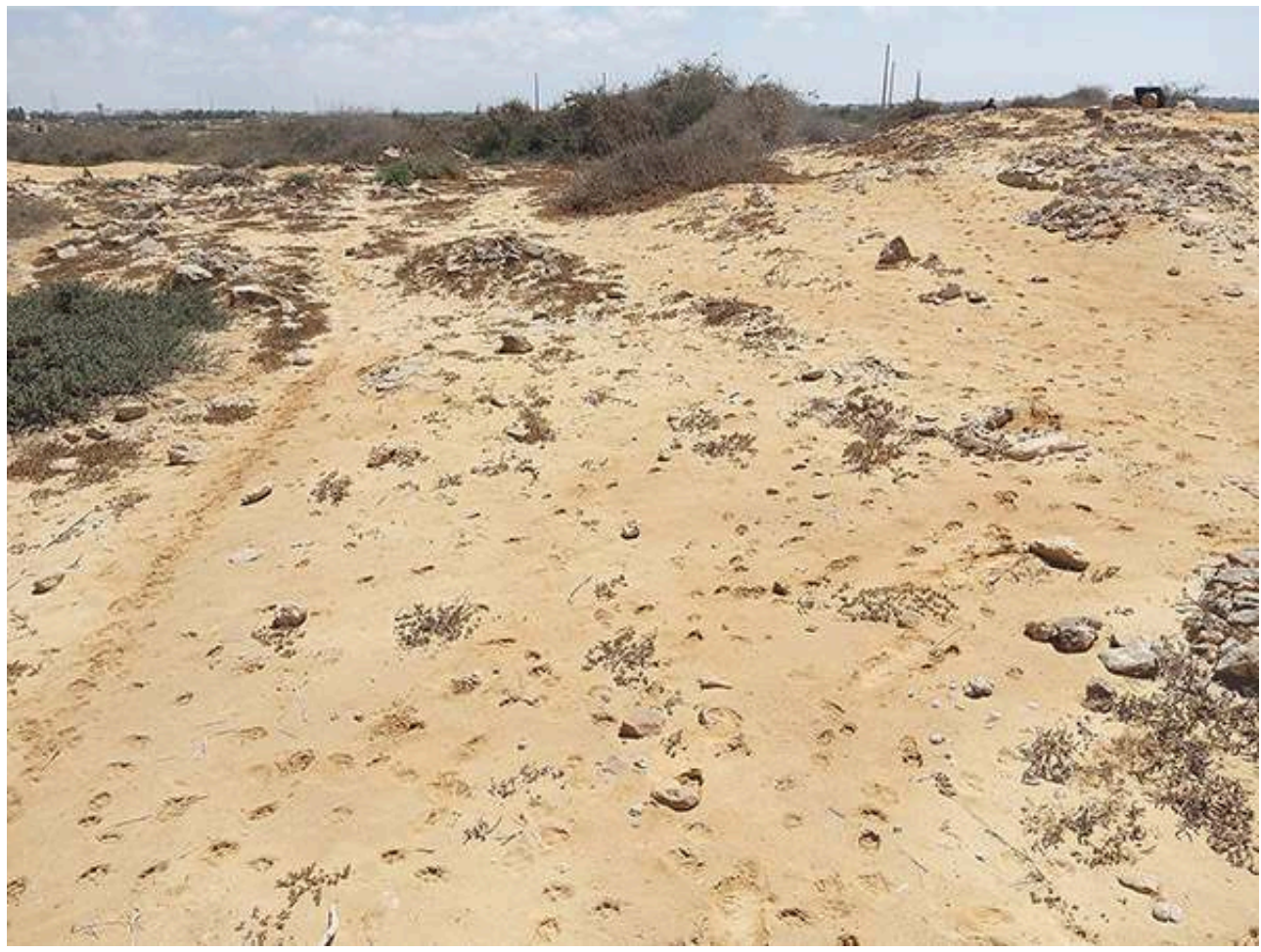

(c) Archives CEAlex. CEAlex_GMR_2020_NDMPF_044

$112 \mathrm{Au}$ centre du site, une zone (secteur 36 ) de près de $150 \mathrm{~m}$ est-ouest sur une soixantaine de mètres nord-sud, relativement plane (fig. 94), excepté quelques proéminences dans la partie orientale, livre en surface de nombreux alignement de blocs appartenant à au moins trois grands bâtiments de plan allongé (fonction de stockage ?) et à d'autres bâtiments de plus petites tailles (fig. 95). On note également la présence de deux puits : un dans le secteur 29, l'autre dans le secteur 30 . 
Fig. 94. Carte archéologique de la Maréotide. GMR0158, secteur 36, vue partielle du secteur, blocs épars et alignement de blocs, vu depuis l'ouest (I. Awad).

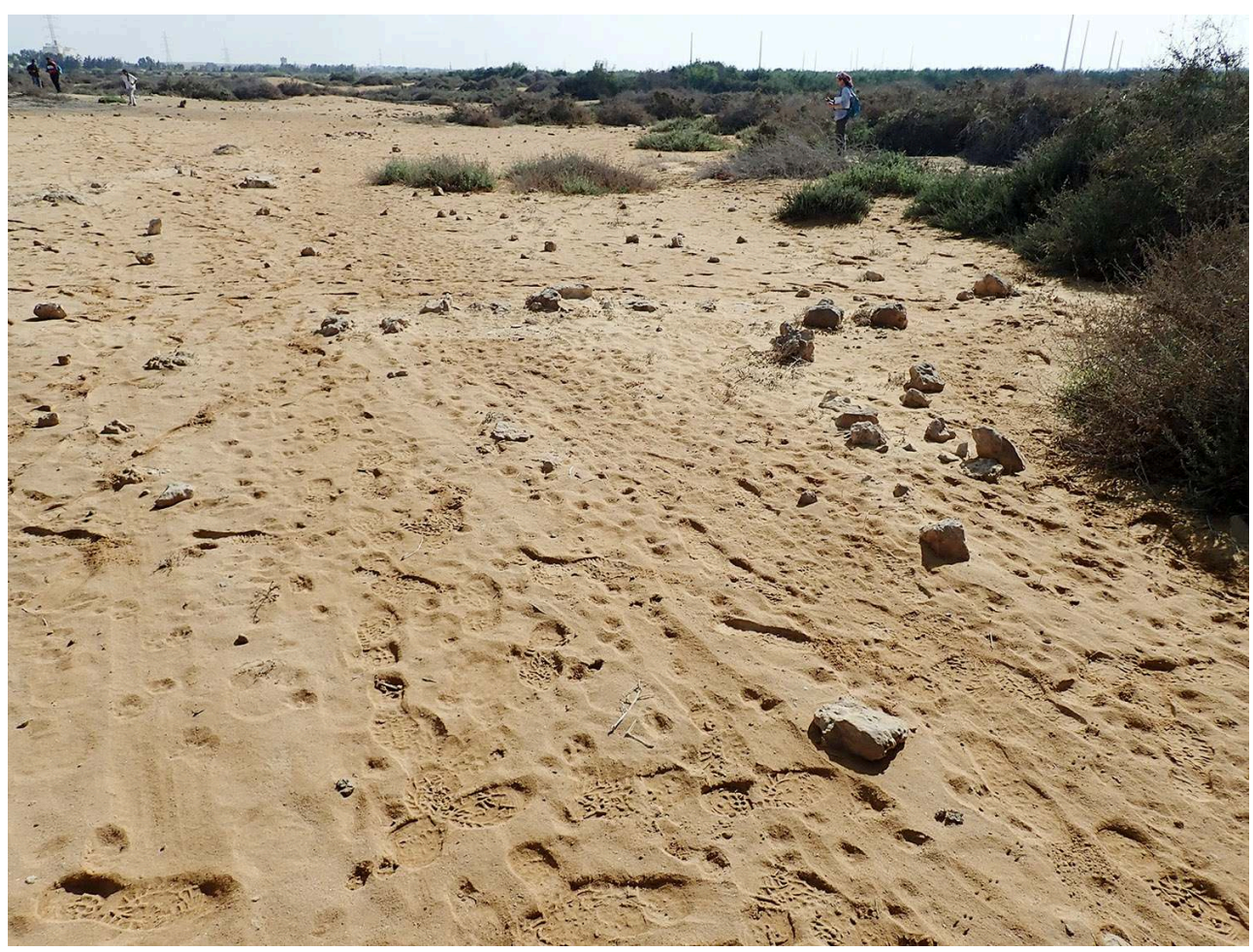

(c) Archives CEAlex. CEAlex_GMR_2020_NDMPF_045

Fig. 95. Carte archéologique de la Maréotide. GMR0158, secteur 36, vue partielle du secteur, blocs épars et alignement de blocs, vu depuis l'ouest (V. Pichot).

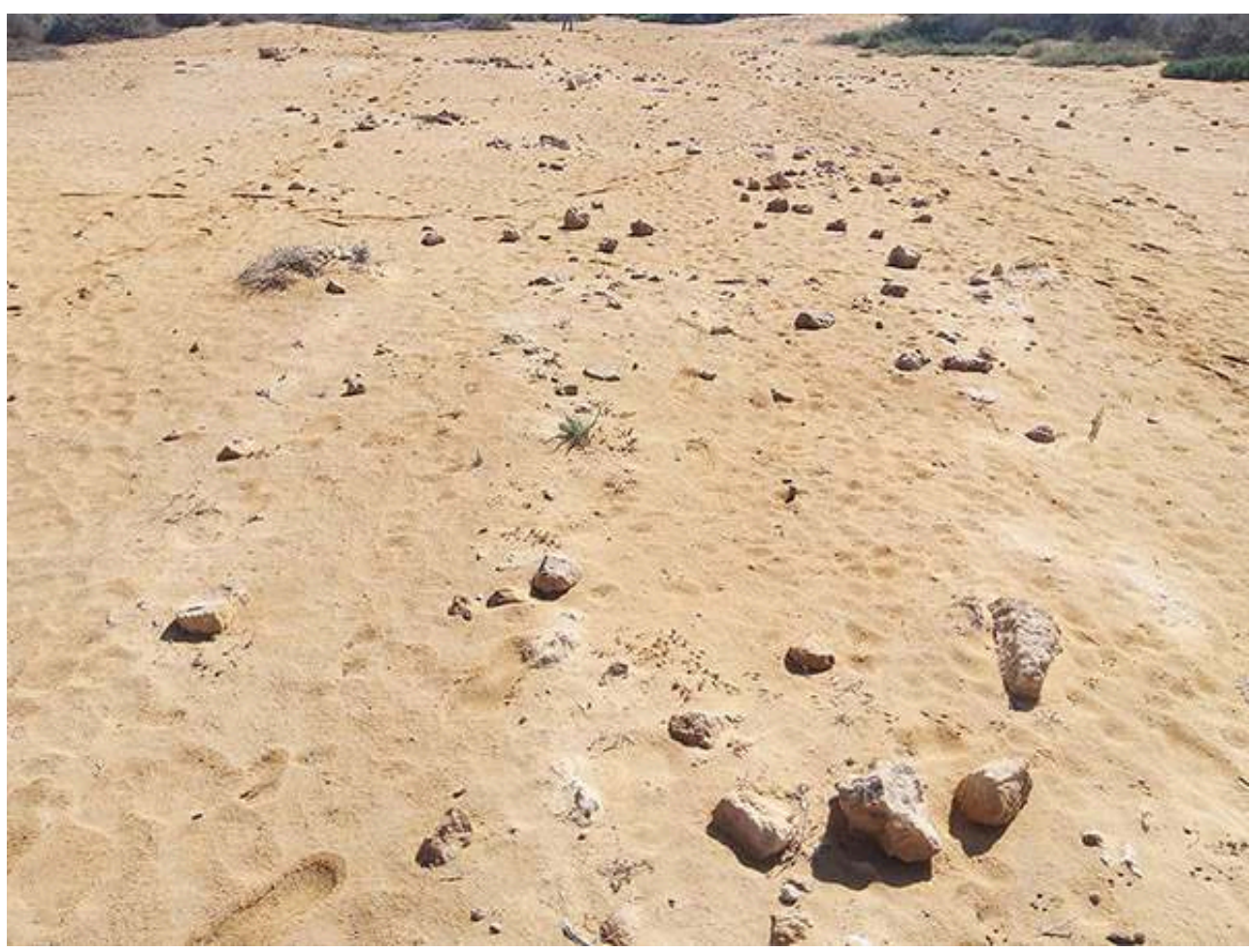

(c) Archives CEAlex. CEAlex_GMR_2020_NDMPF_046 
113 La céramique est assez abondante en surface, mais très fragmentée et érodée par le sel. Plutôt homogène d'un point de vue chronologique, ce matériel (fig. 96) est daté de la fin de l'époque romaine tardive/début de la période islamique $\left(\mathrm{V}^{\mathrm{e}}-\mathrm{VIII}{ }^{\mathrm{e}} \mathrm{s}\right.$. apr. J.-C.) et composé d'amphores de type LRA 1, LRA 4, LRA 5/6, de sigillées LRD et ARSW, de céramique fine du groupe $\mathrm{O}$ d'Assouan, de marmites à bord biseauté, de jattes rubanées, de couvercles-coupelles). La présence d'un dépotoir d'atelier d'amphores LRA 5/6 est à souligner. De très rares fragments plus anciens ont été repérés en surface de manière très ponctuelle (amphore rhodienne et $\mathrm{AE} 2$ ptolémaïques, jatte à marli et $\mathrm{AE} 4 \mathrm{du}$ HautEmpire). Enfin, un petit fragment de pipe de datation plus tardive (vraisemblablement daté d'époque ottomane) a également été retrouvé.

Fig. 96. Carte archéologique de la Maréotide. GMR0158. Matériel provenant du ramassage de surface (période romaine tardive - début de la période islamique). 1-4. Amphores $L R A 5 / 6$ supposées de production locale. 5-6. Amphores importées $L R A$ 1. 7. Jatte à marli rubané. 8. Marmite à bord biseauté. 9. Plat $L R \mathrm{D}$ (A. El Nagar, C. Edwar, M. Fathy).

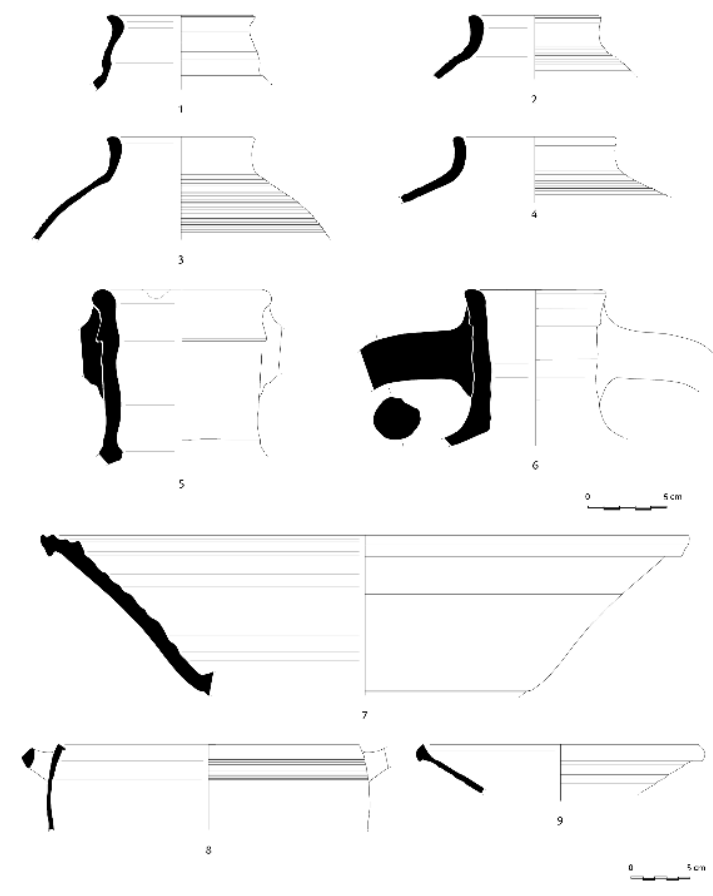

\subsection{Présentation de sites prospectés : la ride $\mathrm{V}$}

\subsubsection{GMR0177 - Shaher Rowhoh}

(fig. 97)

\section{Région de Borg el-Arab}


Fig. 97. Carte archéologique de la Maréotide. Croquis du site GMR0177, Shaher Rowhoh : en bleu, limites actuelles du site et secteurs définis ; en jaune, anomalies (karm) topographiées en 1940 ; en orange, anomalies (karm) visibles sur l'image satellite Landsat 1984 ; en tireté vert, restitution du karm (V. Pichot).

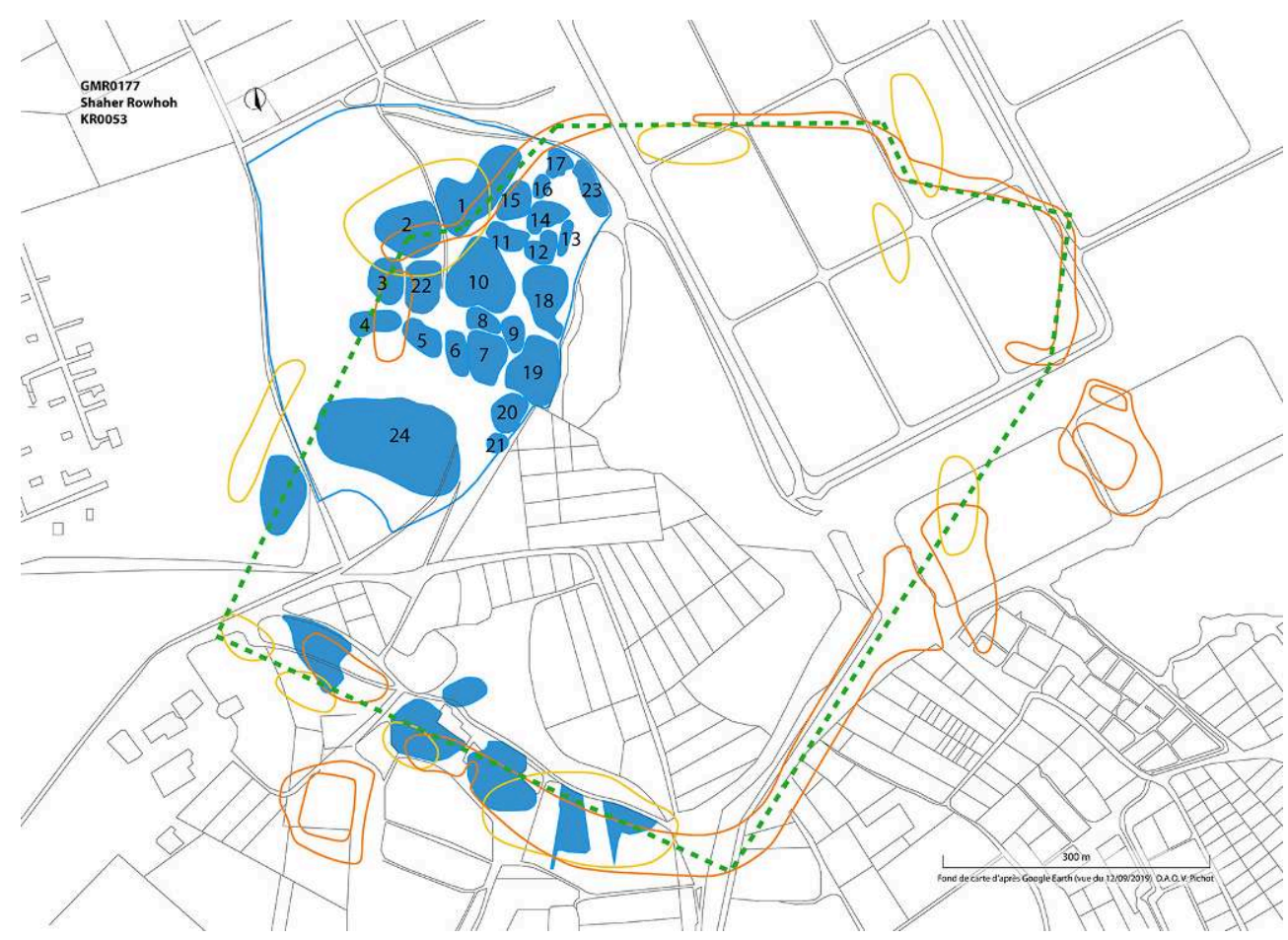

(C) Archives CEAlex. CEAlex_GMR_2020_NDMCN_011

Sur le versant nord de la ride V à 1,7 km à l'est de Zâwyiet el-Iseila (GMR0017), vestiges d'un grand karm (KR0053) topographié sur les cartes du survey de 1914, 1920 et 1940, repéré aussi sur la carte satellite Landsat 1984.

La partie nord-ouest du karm est bien conservée et livre de nombreux vestiges en surface, tandis que la partie nord-est a été totalement détruite par l'installation d'une station d'épuration. La partie sud du karm, bien qu'en partie détruite par la mise en culture de la zone, est encore conservée sur quelques tronçons. La limite orientale, quant à elle, est très détériorée par la présence d'une piste et d'une route asphaltée encadrant une canalisation d'eau usée enterrée. L'intérieur du karm est aujourd'hui totalement nivelé et mis en culture pour la production d'orge. Ce site avait fait l'objet d'une prospection en 2019. L'objectif cette année était de compléter la documentation récoltée sur le terrain pour la partie nord et nord-ouest du site ; la partie sud ayant été déjà documentée en 2019.

116 Le site conservé se compose de deux parties principales :

- La partie nord et nord-ouest du site (fig.98-100), installée sur un promontoire rocheux, forme un grand ensemble qui livre de nombreux bâtiments (fonction domestique et agricole) mêlés à un sédiment argileux beige-orangé. Cette partie du site, composée de 24 secteurs, est située sur le segment ouest de l'enceinte du karm à proximité de son angle nord-ouest. On note que la zone occidentale ne livre en surface que des éléments romains tardifs et islamiques tandis que la zone orientale fournit une fourchette chronologique plus large allant de l'époque hellénistique à la période islamique. Trois cimetières sont implantés à peu de distance du Santon Shaher Rowhoh, 
le cimetière directement associé au Sidi (secteur 1) est un cimetière qui n'est plus utilisé, les deux autres (secteur 14 et secteur 9) (fig. 101) le sont encore.

Fig. 98. Carte archéologique de la Maréotide. GMR0177, secteur nord-ouest du site, partie nord depuis le secteur 6, vue depuis le sud (V. Pichot).

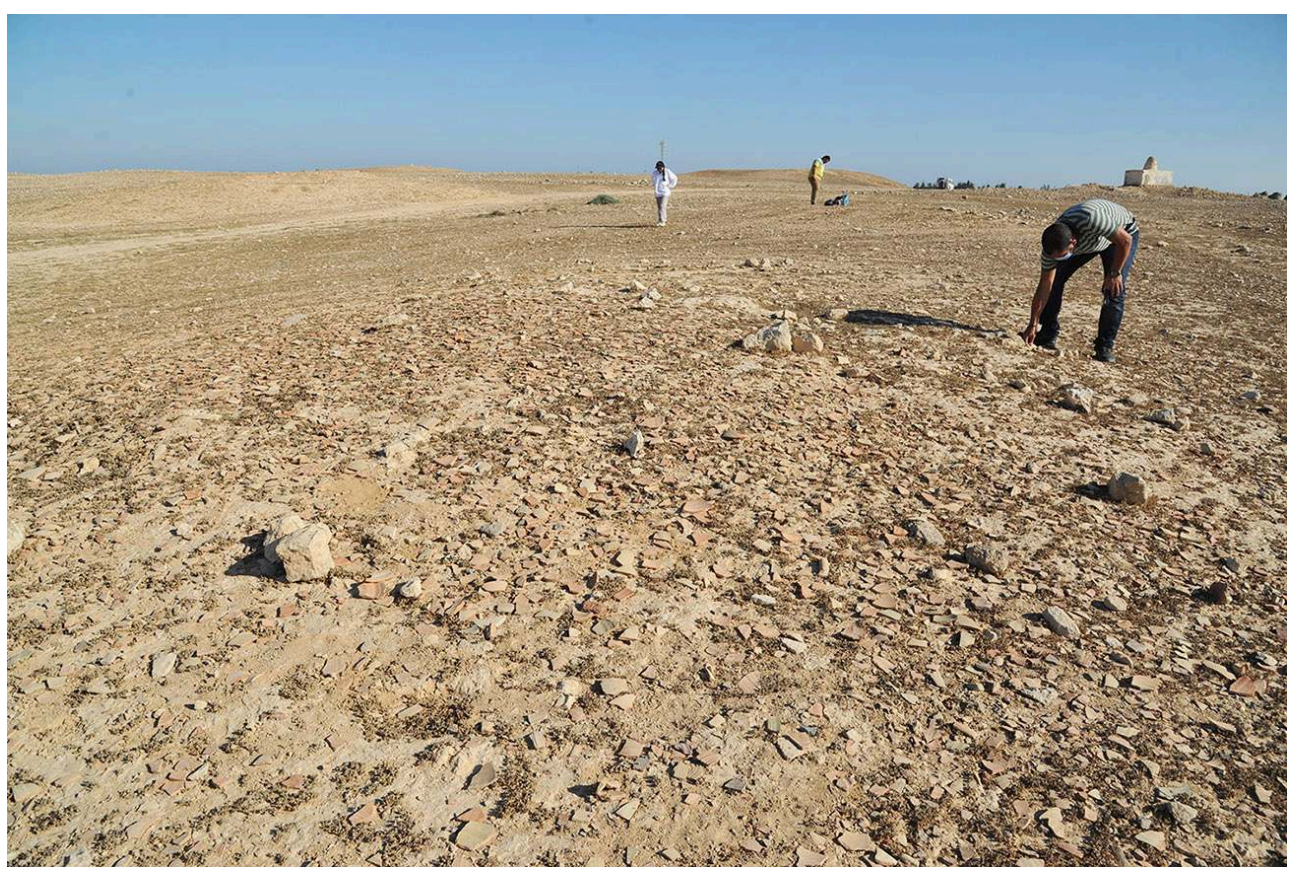

(C) Archives CEAlex. CEAlex_GMR_2020_NDMPF_047

Fig. 99. Carte archéologique de la Maréotide. GMR0177, secteur nord-ouest du site, partie nord depuis le secteur 7, vue depuis le sud (V. Pichot).

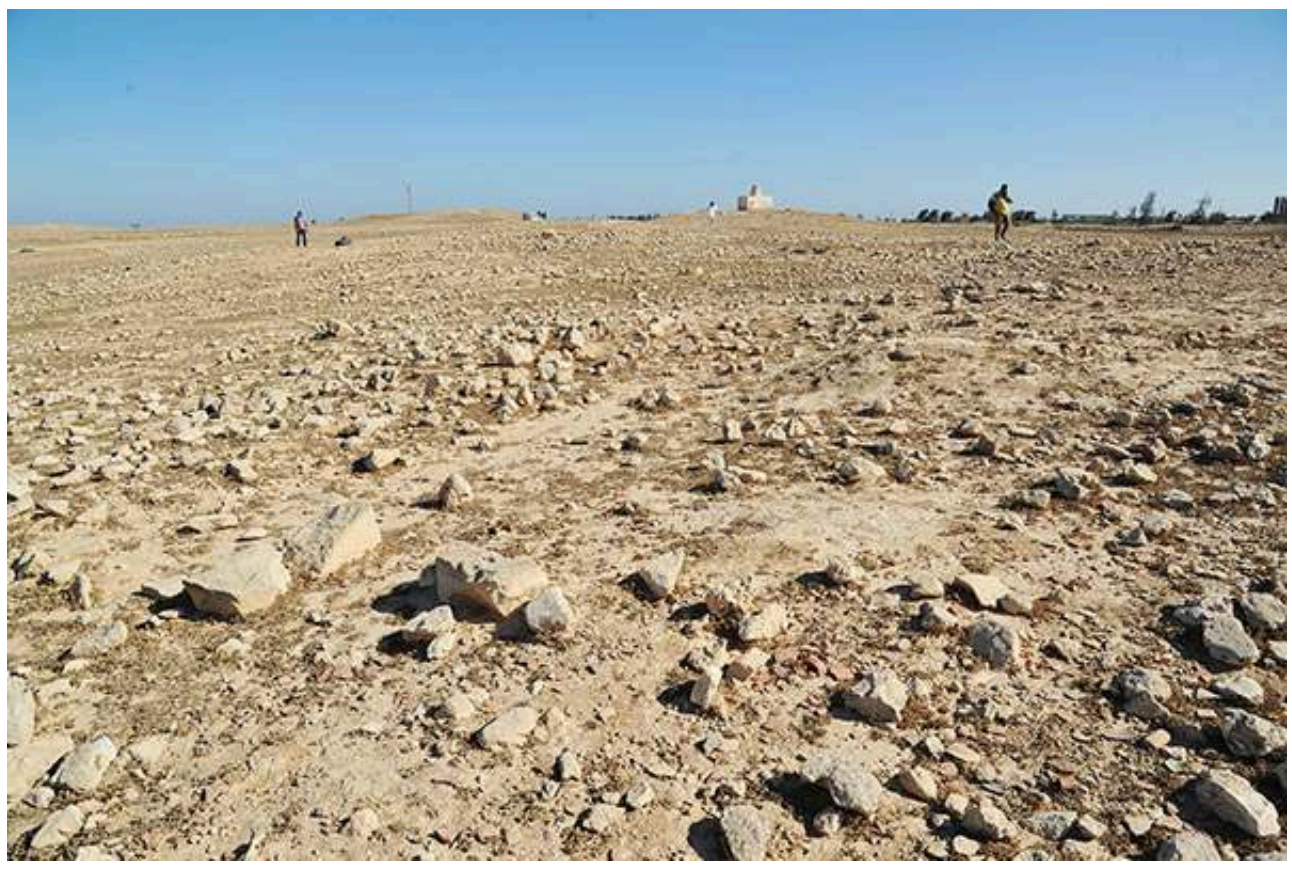

(c) Archives CEAlex. CEAlex_GMR_2020_NDMPF_048 
Fig. 100. Carte archéologique de la Maréotide. GMR0177, secteur nord-ouest du site, partie ouest depuis le secteur 7, vue depuis l'est (V. Pichot).

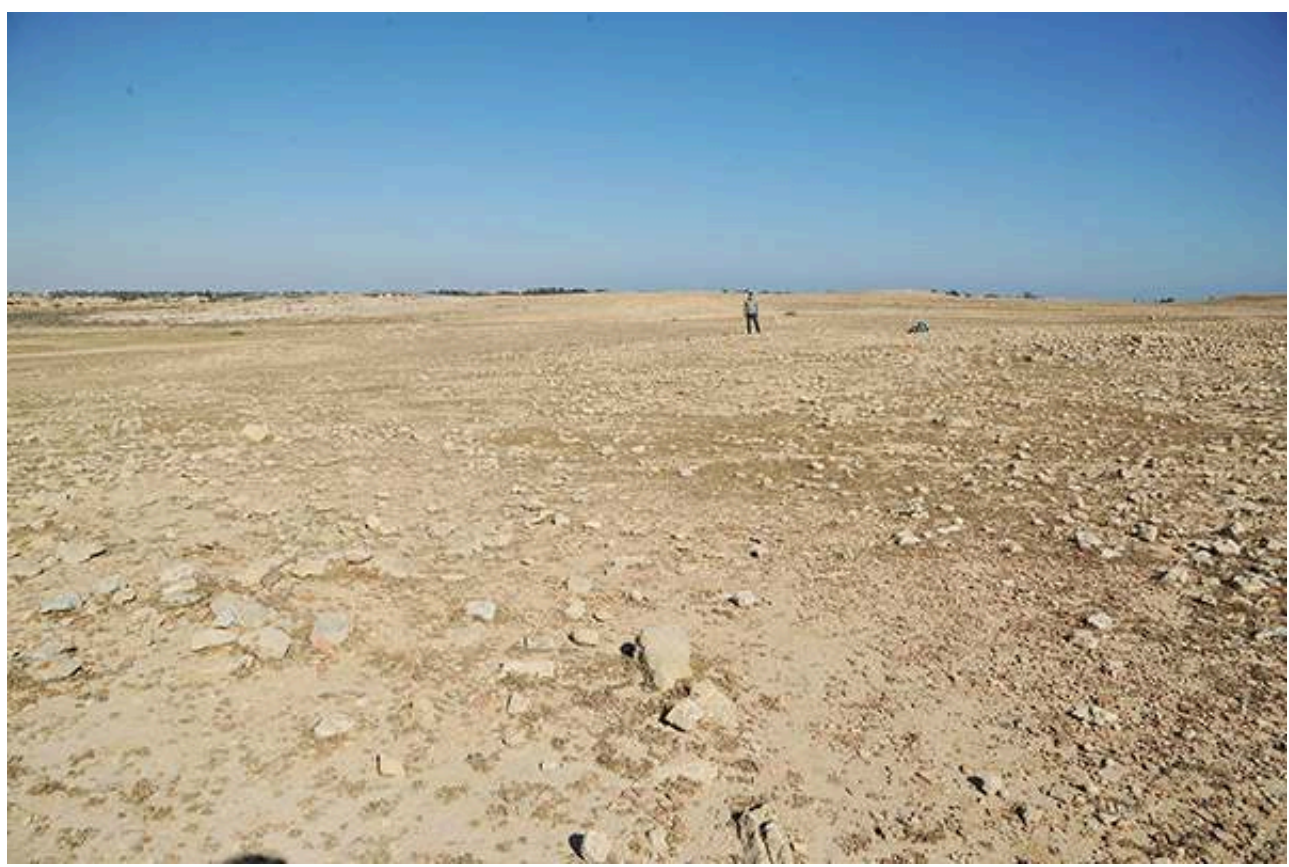

(C) Archives CEAlex. CEAlex_GMR_2020_NDMPF_049

Fig. 101. Carte archéologique de la Maréotide. GMR0177, secteur nord-ouest du site, partie nord depuis le secteur 9, vue depuis le sud (V. Pichot).

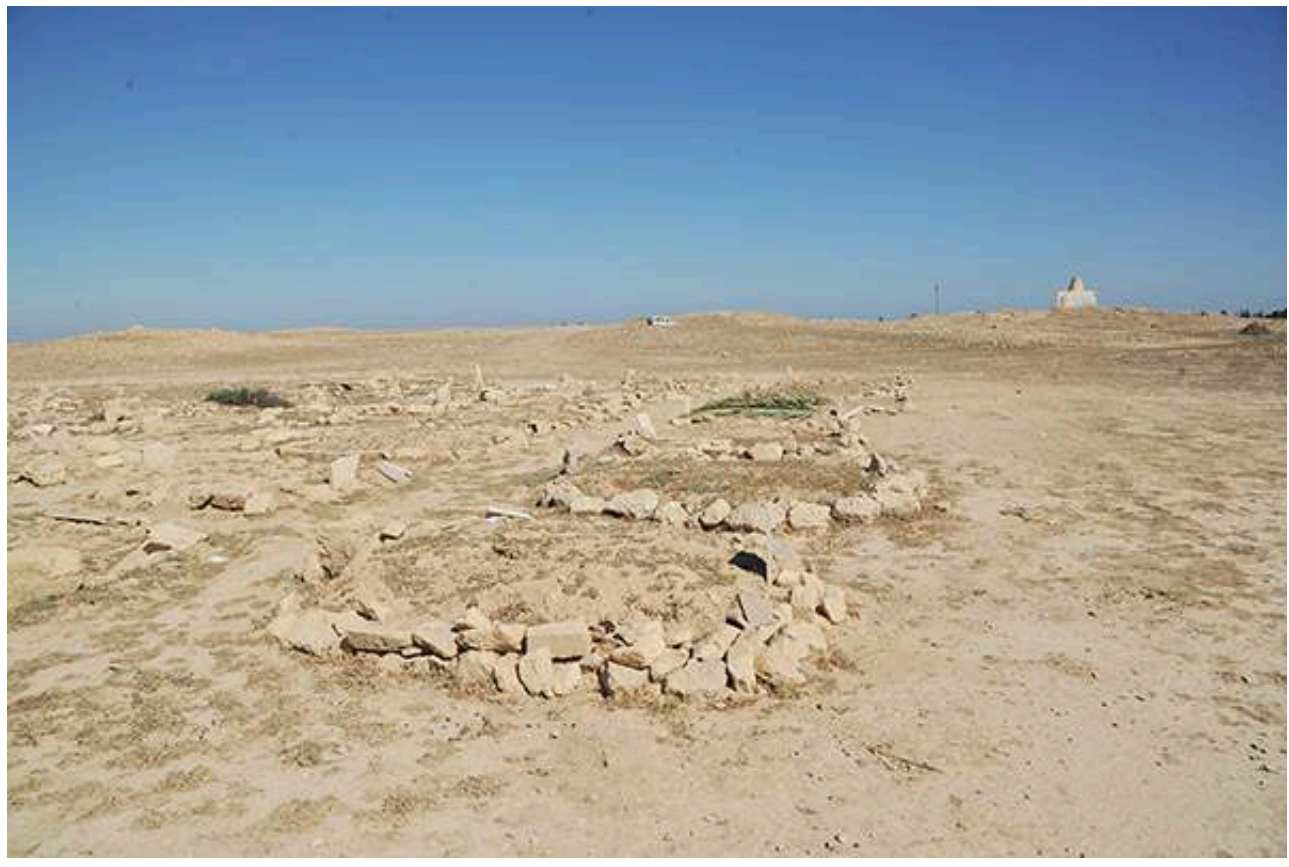

(c) Archives CEAlex. CEAlex_GMR_2020_NDMPF_050

- Le secteur sud du site : à $270 \mathrm{~m}$ du secteur nord-ouest du promontoire rocheux, commencent les premiers vestiges du talus sud de l'enceinte du karm. Ce talus est conservé sous la forme de cinq tronçons de sédiment argileux beige-orangé alignés (orientation nord-ouest - sud-est) (fig. 102). Les trois tronçons occidentaux livrent en 
surface à divers endroits des blocs épars, des fragments d'enduits et de céramique indiquant la présence de structures enfouies. Les deux tronçons orientaux n'offrent, quant à eux, aucun vestige de structure : ils sont exclusivement composés de sédiment argileux beige-orangé.

Fig. 102. Carte archéologique de la Maréotide. GMR0177, secteur sud du site, vue partielle des tronçons du talus sud du karm KR0053, depuis le sud-ouest (A. Simony).

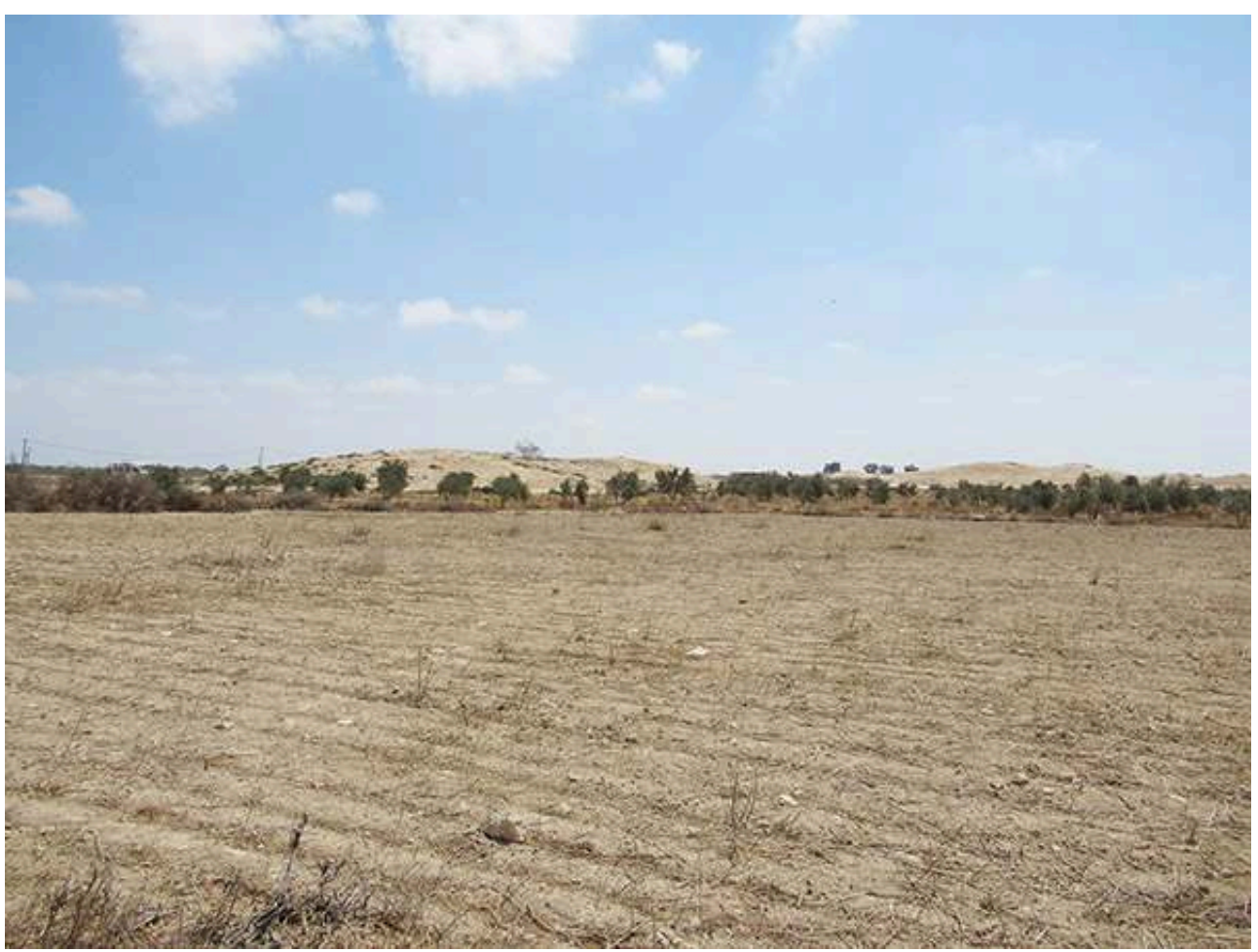

(c) Archives CEAlex. CEAlex_GMR_2020_NDMPF_051

117 L'ensemble du site est très riche en mobilier céramique. Il s'agit majoritairement de céramiques de l'époque romaine tardive/début de la période islamique - nombreuses LRA 5/6, forme fermée à décor peigné, AE 8, LRA 1, LRA 4, poêle, cruche à décor incisé " Abou Mina », nombreux fragments de $L R \mathrm{D}$, quelques $A R S W$, céramiques du groupe $\mathrm{W}$, du groupe $\mathrm{O}$ et $\mathrm{du}$ groupe $\mathrm{K}$, marmite importée de Cilicie, gargoulette à décor peint violine, jattes à marli rubané, forme fermée à pâte calcaire locale et décor digité. Le site livre aussi des éléments des périodes antérieures - anse timbrée rhodienne portant le buste d'Hélios au centre daté de c. 146 - c. 108 av. J.-C., ES A et lopas pour la période ptolémaïque ; amphores $\mathrm{AE} 4, \operatorname{AE} 3$, cratère d'imitation chypriote, plat de cuisson du Haut-Empire - et postérieures - glaçures islamiques, formes fermées à décor peigné datées des $\mathrm{IX}^{\mathrm{e}}-\mathrm{XI}{ }^{\mathrm{e}}$ siècles.

\subsection{Conclusion}

118 Notre recherche menée dans la région de la Maréotide montre bien toute la richesse archéologique de cette partie de la chôra alexandrine encore largement méconnue jusque récemment. Au fil des prospections, les nouvelles données permettent de nous familiariser avec cette région en cours de destruction, mais qui offre encore de nombreux sites parfois de grande taille, comme Karm el-Qatf, Shaher Rowhoh ou 
encore Sidi Soliman el-Tayer, révélant ainsi ce terroir d'Alexandrie éminemment agricole sur la longue diachronie.

La prochaine mission qui se déroulera en automne 2021 aura pour objectif d'une part de continuer la documentation de la région qui se développe au sud de la ride III, et plus particulièrement le secteur qui se développe à l'ouest d'Huwareya, et d'autre part d'effectuer une expertise plus poussée de certains karms en effectuant des carottages pour connaître le potentiel en terme archéobotanique de ces structures agricoles.

\section{Fouilles sous-marines sur le site du Phare}

Ismaël Awad, Mohamed Abdelaziz, Mohamed Elsayed, Isabelle Hairy, Philippe Soubias Responsable scientifique du site du Phare : Isabelle Hairy.

Chef d'opération hyperbare : Mohamed Elsayed.

Participants : Ismaël Awad et Cécile Shaalan (service de topographie), Philippe Soubias (traitement photogrammétrique et responsable du service plongée du CEAlex), Mohamed Abdelaziz (traitement photogrammétrique), Sherine El Sayed, Ismail El Sayed et Aly Sayed Aly Mohamed Ahmed El Dabaa (responsables techniques), Ashraf Hussein Gomaa Aly Salam, Tamer Mohamed Abdel Salam Bassiouny, Wael Mostafa Mohamed, Hassan Yasser Galal Abdel Rehim Aly (plongeurs du CEAlex), Ahmed Abdel Fattah Rashwan, Mahmoud Metwali Khalil et cinq ouvriers (équipe terrestre).

Autorité nationale présente: Le MoTA était représenté par Atef Ibrahim Aly (Département central d'archéologie sous-marine) et par Yaser Abd Elrazik (Département d'archéologie sous-marine de la Mer Rouge).

L'officier de la Marine nationale Égyptienne était le sergent-chef Samy Ibrahim Hassan et le sergent Hossam Eldeen Yasser.

Partenariat institutionnel: Les fouilles sous-marines du CEAlex bénéficient $d u$ soutien de l'Honor Frost Foundation.

La campagne sous-marine s'est déroulée entre le 27 octobre et le 26 novembre 2020 . L'équipe s'est mise à l'eau le $1^{\text {er }}$ novembre après quelques jours de préparation terrestre en commençant par quelques jours de prospection pour repérer les zones de travail en photogrammétrie, ainsi que les blocs à photographier. Avec une météo variable, le mois de mission a compté de nombreuses journées de houle moyenne à forte et une visibilité réduite, limitant ainsi le nombre de jours travaillés ( 15 jours plongés sur 20 jours ouvrés de mission). La construction du brise-lames à l'ouest du site commence à apporter une protection aux plongeurs lors de certains épisodes de houle de direction ouest-nord-ouest.

Étant donné la courte durée de la mission, le programme de travail a été centré sur les deux points essentiels de l'étude du site : la prise de photos pour le modèle numérique de surface (MNS) et la photographie de fragments ou pièces d'architecture et de statuaire en vue de leur modélisation 3D par photogrammétrie numérique automatique.

\subsection{La numérisation du site subaquatique}

Le travail autour du modèle numérique de surface (MNS) a fait l'objet de plusieurs missions avant celle-ci, en 2014, 2015, 2016, 2018 et 2019 (fig. 103). En 2020, l'avancement du MNS a été conduit sur deux zones différentes du site : dans un premier 
temps, au nord de la zone 1 sur une surface d'environ $1687 \mathrm{~m}^{2}$ (fig. 103, 2020-A) et dans un second temps, au sud de la zone 2 sur une surface d'environ $686 \mathrm{~m}^{2}$ (fig. 103, 2020-B). Cette seconde zone aurait dû être plus étendue, mais la complexité du relief associée à une mauvaise météo a empêché de finir le travail.

Fig. 103. Site du Phare. Carte montrant l'avancement de l'acquisition du modèle numérique de surface par année, de 2014 à 2020 (I. Hairy).

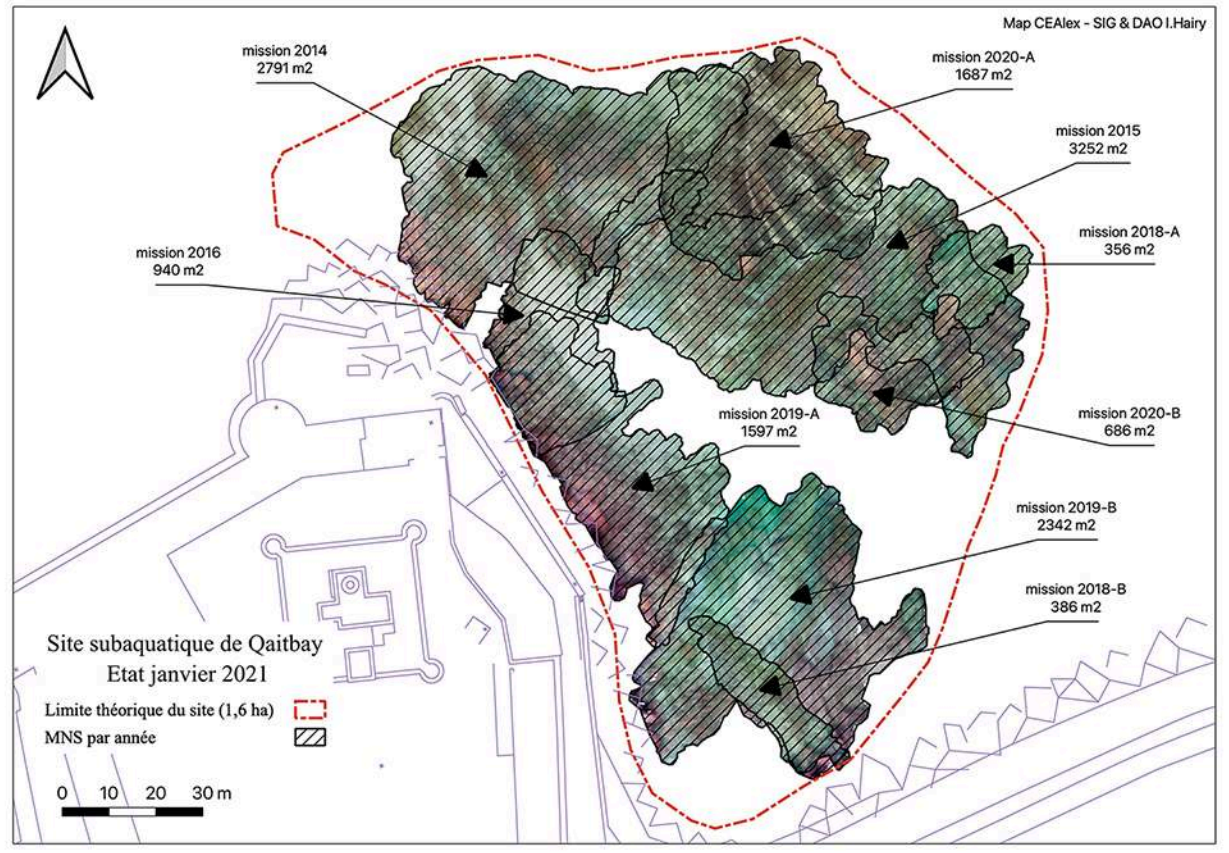

(c) Archives CEAlex. CEAlex_QB_2020_NDMCN_001

Avant les opérations de photographie ${ }^{25}$, les deux zones ont été préparées par l'implantation de 20 points d'appui (GCP26), numérotés de 39 à 59, répartis sur toute leur surface. Les GCP sont repérés sous l'eau par un piquet en métal profondément ancré dans le substrat et portant un numéro sur support plastique ${ }^{27}$ (fig. 104). Cette méthode initiée en 2014 permet de conserver sur la durée la plupart des points implantés. Les 20 points 2020 ont été levés par tachéomètre lors d'une intervention menée par le service de topographie du CEAlex (fig. 105). Chaque point a fait l'objet de 10 mesures à partir de la station 2206 située sur le quai à l'ouest du fort Qaitbay. Le nombre important de mesures réduit de façon significative les erreurs et permet d'approcher au plus près les coordonnées "réelles» du GCP. Une moyenne arithmétique est déterminée dans Autocad à partir du polygone constitué par les mesures faites sur chaque point (après exclusion des points aberrants). Cette moyenne arithmétique est tempérée graphiquement pour déterminer les coordonnées planimétriques retenues, en corrigeant manuellement la déviation commune des points générée par la houle ou le courant. 
Fig. 104. Site du Phare. Photo d'un point d'appui (GCP) implanté en 2020 (P. Soubias).

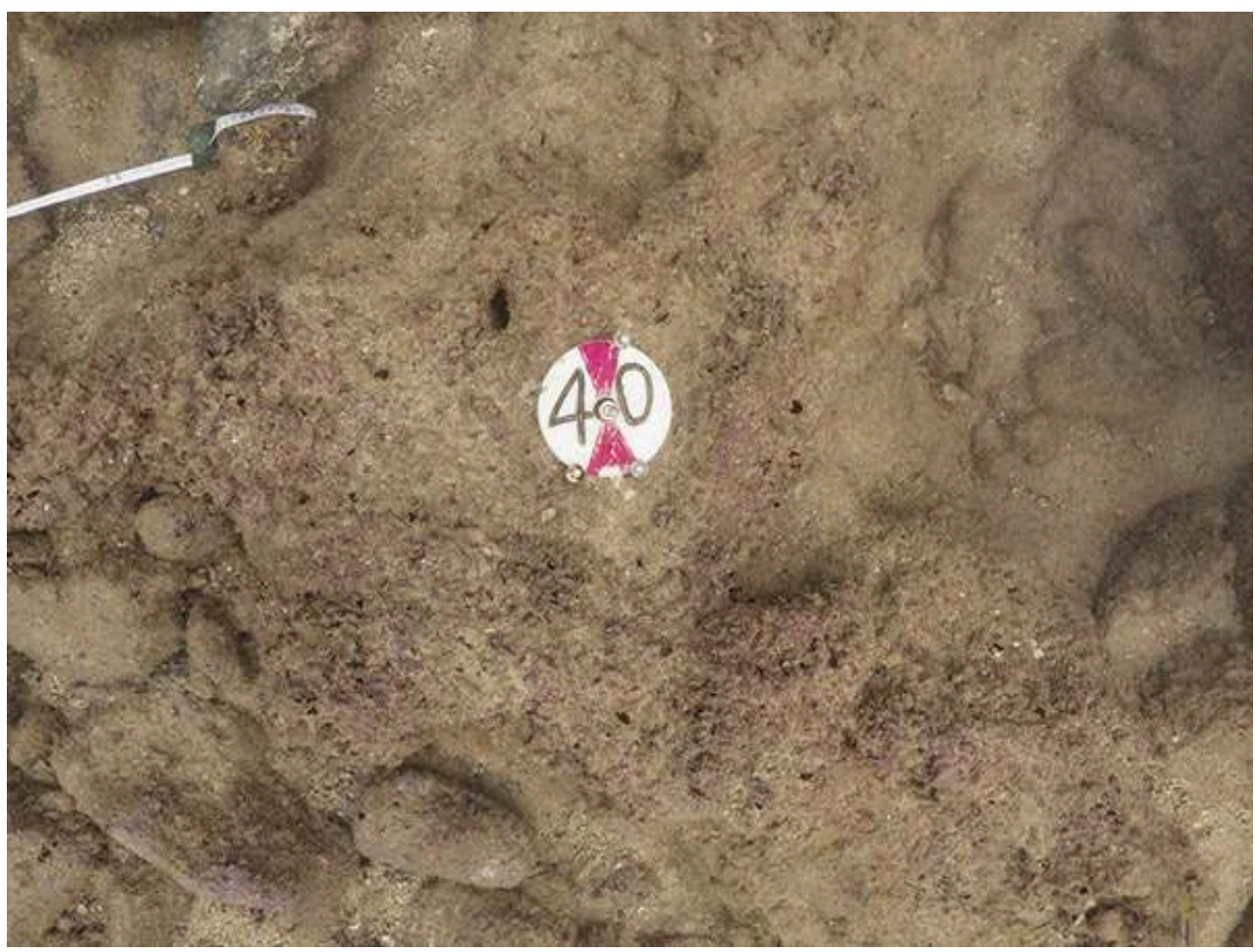

(c) Archives CEAlex. CEAlex_QB_2020_NDMCN_002

Fig. 105. Site du Phare. Position des stations implantées par le service de topographie du CEAlex et GCP implantés par Mohamed Elsayed lors de la mission 2020 (I. Awad).

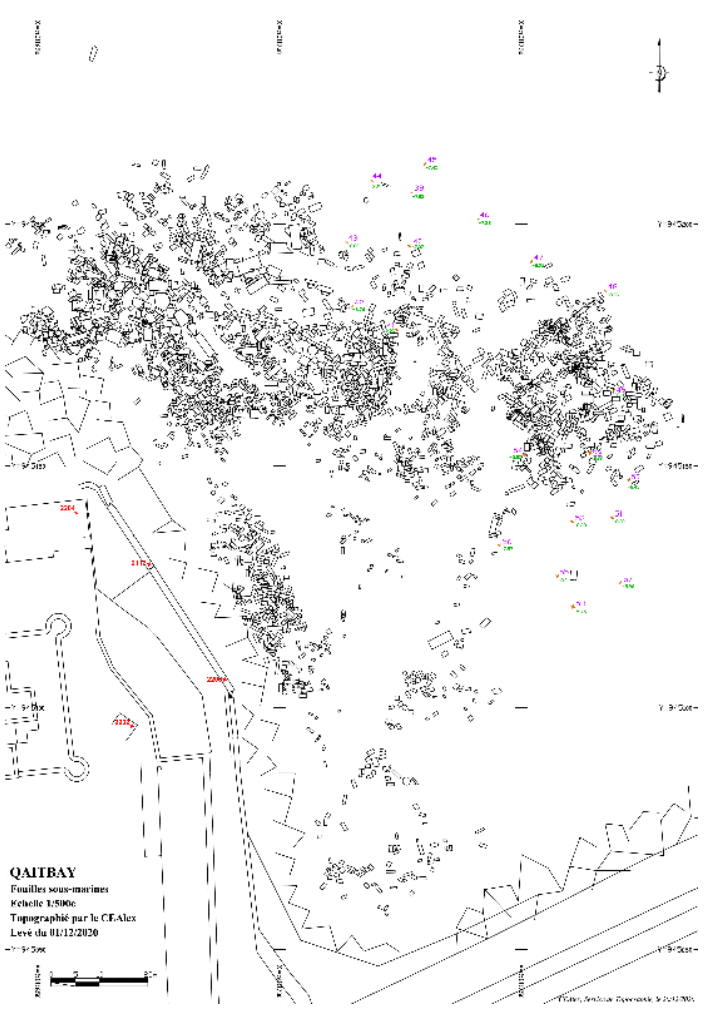

(c) Archives CEAlex. CEAlex_QB_2020_NDMCN_003 
124 L'altitude moyenne est calculée pour chaque point à partir des visées retenues pour la planimétrie (fig. 106-107). 17 GCP sur les 20 levés ont été conservés pour la transformation et le géoréférencement du modèle numérique de surface ; les points 46 , 47 et 55 ont été exclus en raison d'une trop grande imprécision.

Fig. 106. Site du Phare. Contrôle des erreurs sur les coordonnées des GCP sur les ortophotoplans 2020 : secteur 2020A (M. Abdelaziz).

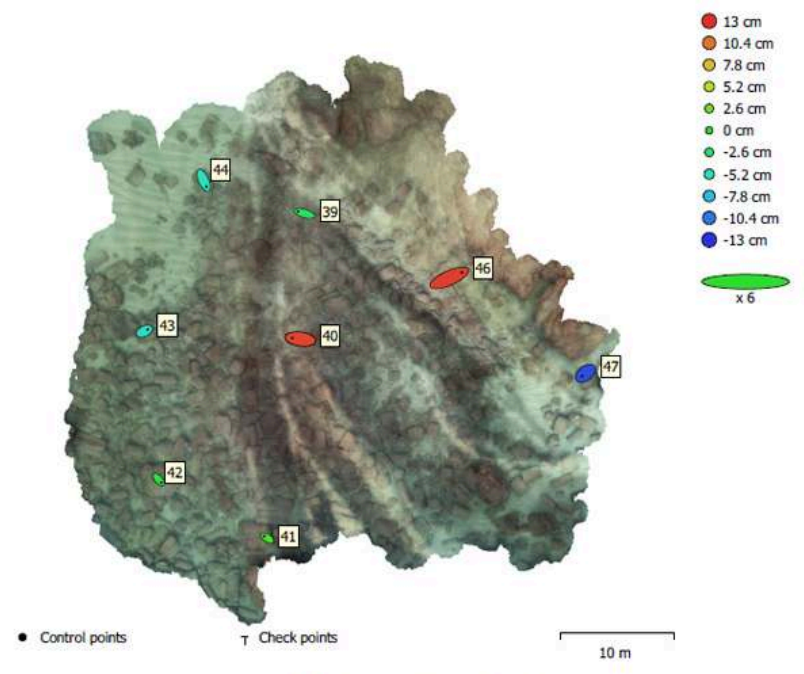

GCP locations and error estimates.

$Z$ error is represented by ellipse color. X,Y errors are represented by ellipse shape. Estimated GCP locations are marked with a dot or crossing.

\begin{tabular}{|l|l|l|l|l|l|}
\hline Count & X error $\mathbf{( c m )}$ & Y error $(\mathbf{c m})$ & Z error $(\mathbf{c m})$ & XY error $(\mathbf{c m})$ & Total $(\mathbf{c m})$ \\
\hline 8 & 18.2553 & 10.1228 & 7.95621 & 20.8741 & 22.3389 \\
\hline
\end{tabular}

Table 5. Control points RMSE.

$X$ - Easting, $Y$ - Northing, $Z$ - Altitude.

(C) Archives CEAlex. CEAlex_QB_2020_NDMCN_004 
Fig. 107. Site du Phare. Contrôle des erreurs sur les coordonnées des GCP sur les ortophotoplans 2020 : secteur 2020B (M. Abdelaziz).

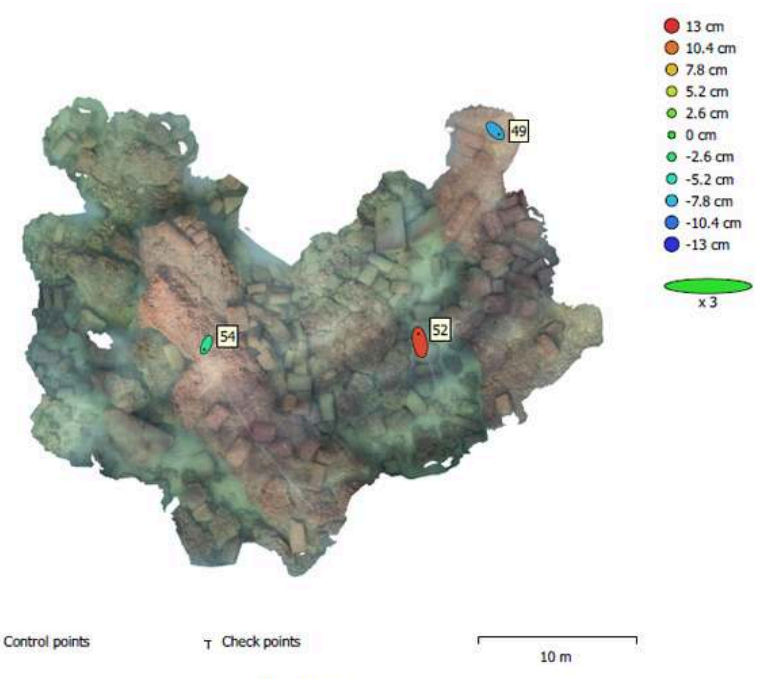

GCP locations and error estimates.

$Z$ error is represented by ellipse color. $X, Y$ errors are represented by ellipse shape. Estimated GCP locations are marked with a dot or crossing.

\begin{tabular}{|l|l|l|l|l|l|}
\hline Count & X error $\mathbf{( c m )}$ & Y error $\mathbf{( c m )}$ & Z error $\mathbf{( c m )}$ & XY error $\mathbf{( c m )}$ & Total $(\mathbf{c m})$ \\
\hline 3 & 10.9851 & 25.5284 & 8.77158 & 27.7916 & 29.143 \\
\hline
\end{tabular}

Table 4. Control points RMSE.

$X$ - Easting, $Y$ - Northing, Z - Altitude.

(c) Archives CEAlex. CEAlex_QB_2020_NDMCN_005

Avant chaque session de prises de vue, des mètres ruban et des règles graduées ont été placés sur et autour des parties à photographier, une méthode qui tend à multiplier les repères en raison de la mauvaise visibilité générale sur le site. Le plongeur photographe se sert de ces repères pour tenir son "plan de vol » (fig. 108-109) auquel il associe la boussole les jours de grand brouillard. 
Fig. 108. Site du Phare. Plan de vol de l'acquisition photographique des zones 2020 réalisée par Mohamed Elsayed : secteur 2020A. (M. Abdelaziz).

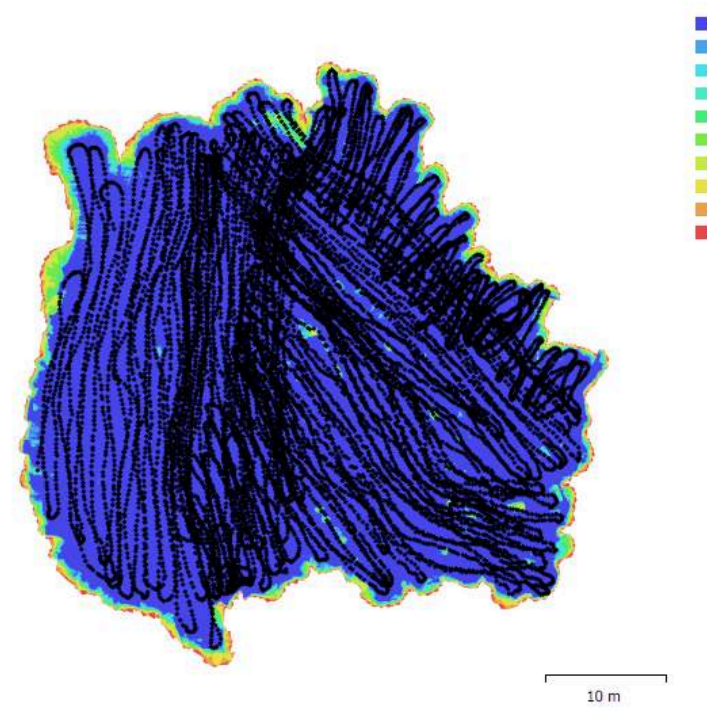

Camera locations and image overlap.

$\begin{array}{llll}\text { Number of images: } & 14,692 & \text { Camera stations: } & 14,692 \\ \text { Flying altitude: } & 1.82 \mathrm{~m} & \text { Tie points: } & 31,982,124 \\ \text { Ground resolution: } & 0.485 \mathrm{~mm} / \mathrm{pix} & \text { Projections: } & 117,189,045 \\ \text { Coverage area: } & 1.65 \mathrm{e}+03 \mathrm{~m}^{2} & \text { Reprojection error: } & 1.54 \text { pix }\end{array}$

(C) Archives CEAlex. CEAlex_QB_2020_NDMCN_006 
Fig. 109. Site du Phare. Plan de vol de l'acquisition photographique des zones 2020 réalisée par Mohamed Elsayed : secteur 2020B (M. Abdelaziz).

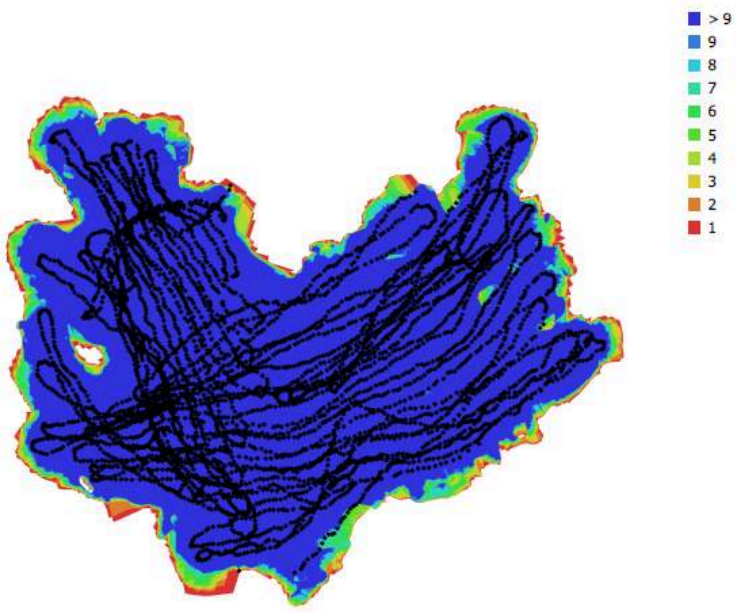

$10 \mathrm{~m}$

Camera locations and image overlap.

$\begin{array}{llll}\text { Number of images: } & 5,721 & \text { Camera stations: } & 5,718 \\ \text { Flying altitude: } & 1.91 \mathrm{~m} & \text { Tie points: } & 11,616,704 \\ \text { Ground resolution: } & 0.764 \mathrm{~mm} / \mathrm{pix} & \text { Projections: } & 39,491,849 \\ \text { Coverage area: } & 684 \mathrm{~m}^{2} & \text { Reprojection error: } & 1.6 \mathrm{pix}\end{array}$

(c) Archives CEAlex. CEAlex_QB_2020_NDMCN_007

La photogrammétrie de la zone couverte lors de cette mission a été traitée dans Metashape (logiciel de photogrammétrie) à partir d'un total de 20413 images (14 692 images en zone A et 5721 images en zone B). Deux orthophotographies ont été produites et insérées au SIG du site, géré sous QGIS ${ }^{28}$ (voir fig. 106-107, 2020-A et 2020B). Créés dans Metashape, des modèles numériques d'élévation montrent le dénivelé des deux nouvelles zones (fig. 110-111). 
Fig. 110. Site du Phare. Modèle numérique d'élévation des deux zones traitées en photogrammétrie en 2020 : secteur 2020A (M. Abdelaziz).

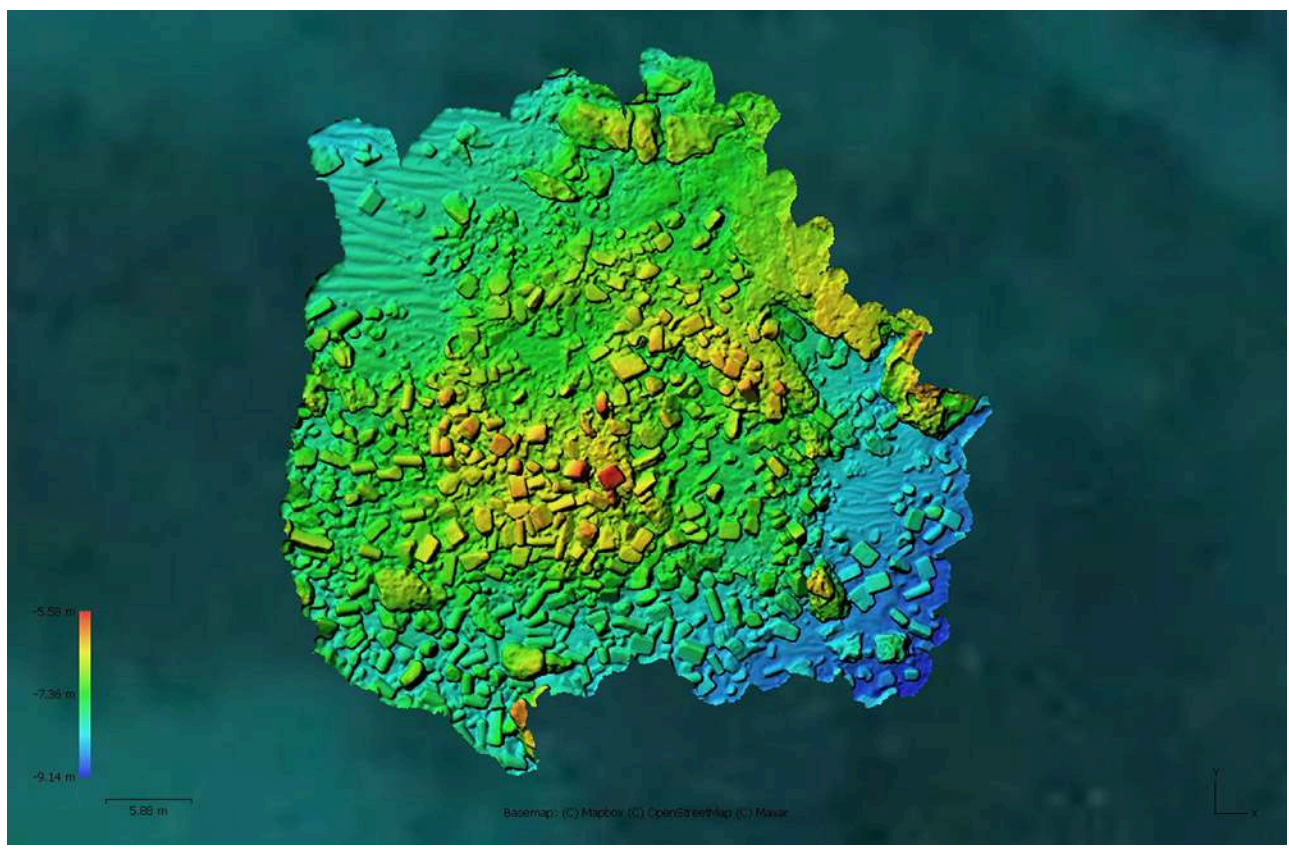

(c) Archives CEAlex. CEAlex_QB_2020_NDMCN_008

Fig. 111. Site du Phare. Modèle numérique d'élévation des deux zones traitées en photogrammétrie en 2020 : secteur 2020B (M. Abdelaziz).

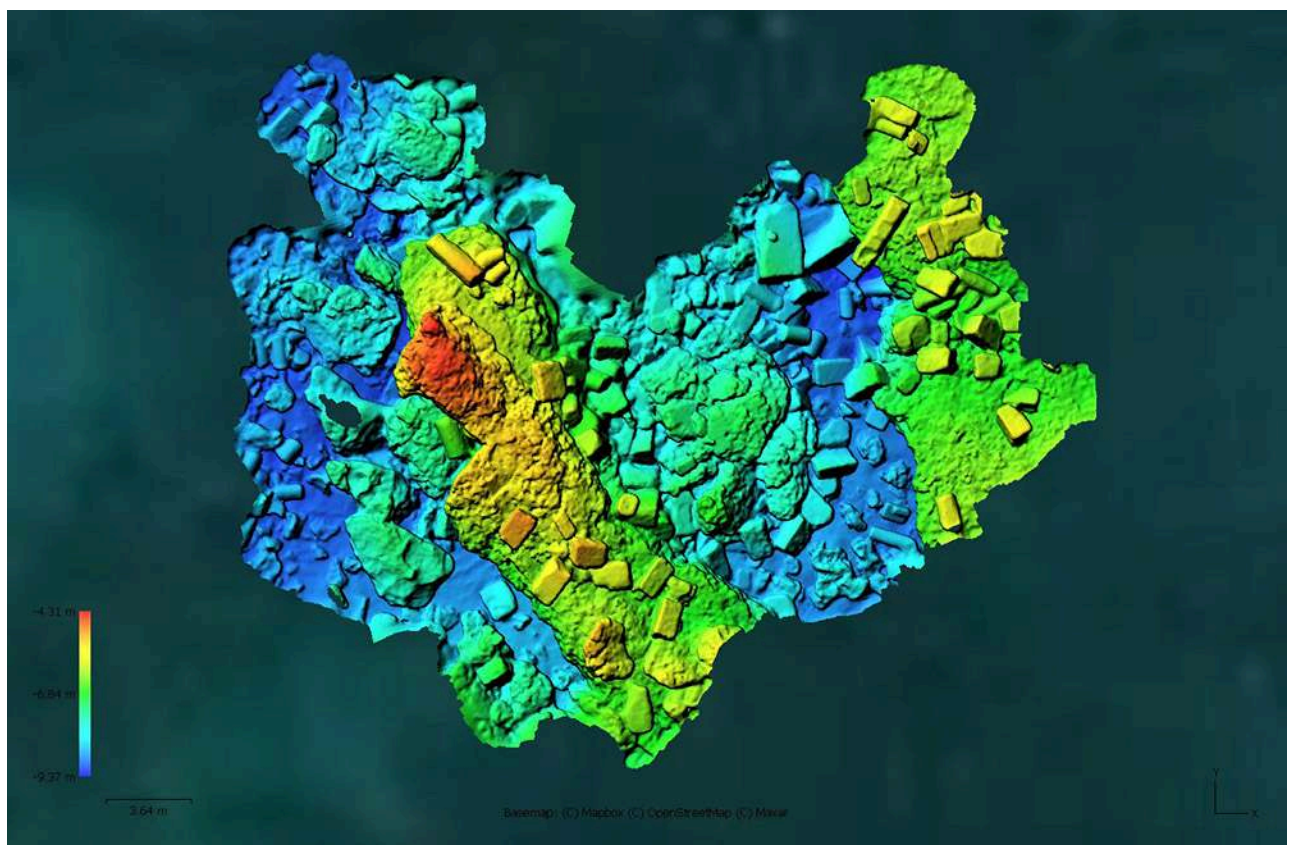

(c) Archives CEAlex. CEAlex_QB_2020_NDMCN_009

127 Le MNS couvre à présent une surface totale de c. $11181 \mathrm{~m}^{2}$ (contre $10250 \mathrm{~m}^{2}$ en 2019), ce qui représente environ $70 \%$ du site archéologique qui a été réévalué en 2019 à 1,6 hectare $^{29}$ (fig. 112). 
Fig. 112. Site du Phare. Vue du modèle numérique de surface fin 2020 (Mohamed Abdelaziz, Mohamed Elsayed).

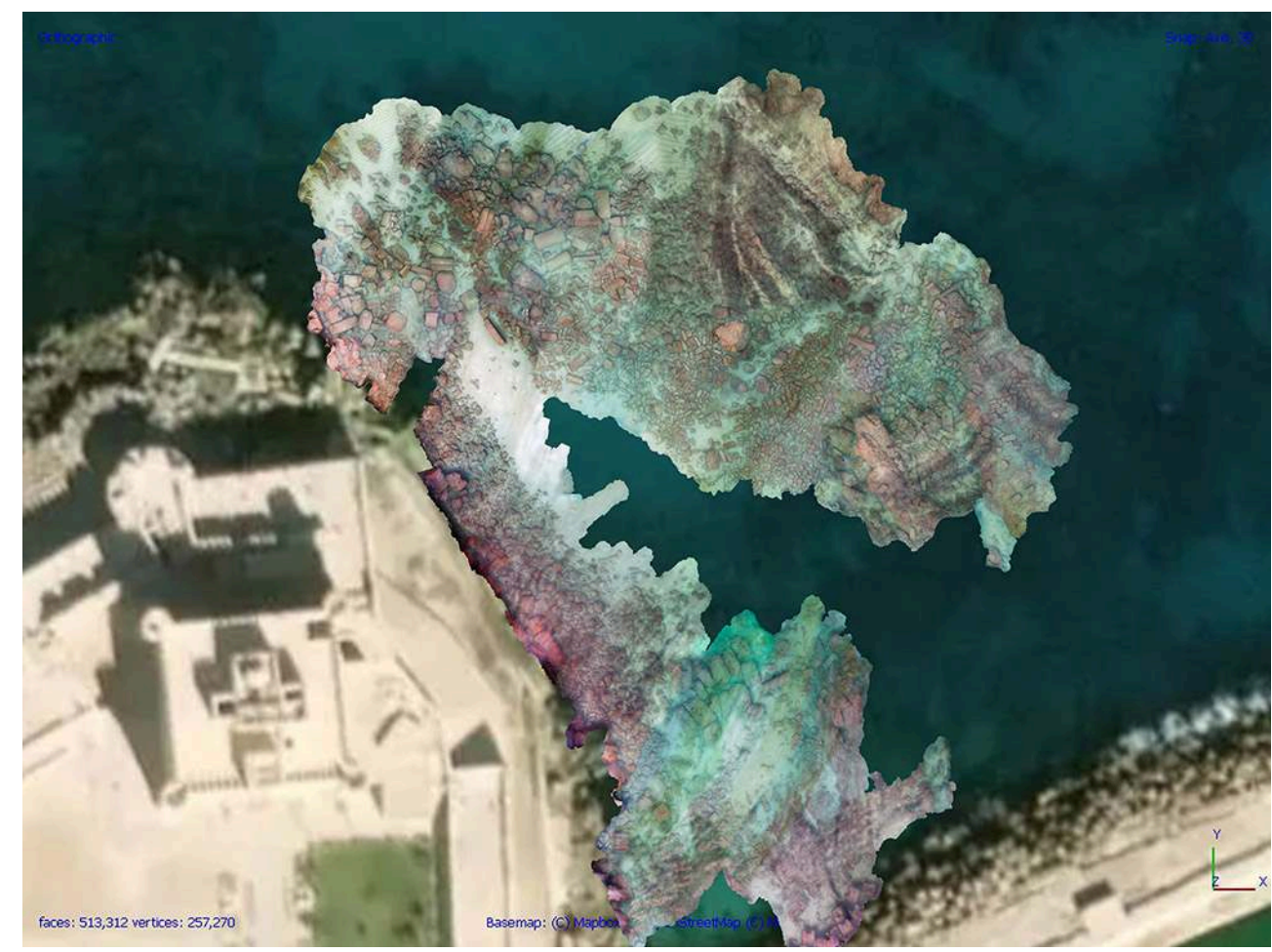

(c) Archives CEAlex. CEAlex_QB_2020_NDMCN_010

\subsection{Photogrammétrie de pièces ou fragments d'architecture et de statuaire}

Huit fragments d'architecture ont été repérés, nettoyés (fig. 113) et photographiés lors de cette mission afin d'en créer des copies numériques. L'acquisition n'a pas été complète pour tous les blocs, en raison de la mauvaise météo, du poids du bloc ou de sa position sur le site. Quand cela a été possible, le bloc a été déplacé et retourné pour l'acquisition de toutes ses faces (fig. 114). 
Fig. 113. Site du Phare. Nettoyage d'un bloc avant photographie (P. Soubias).

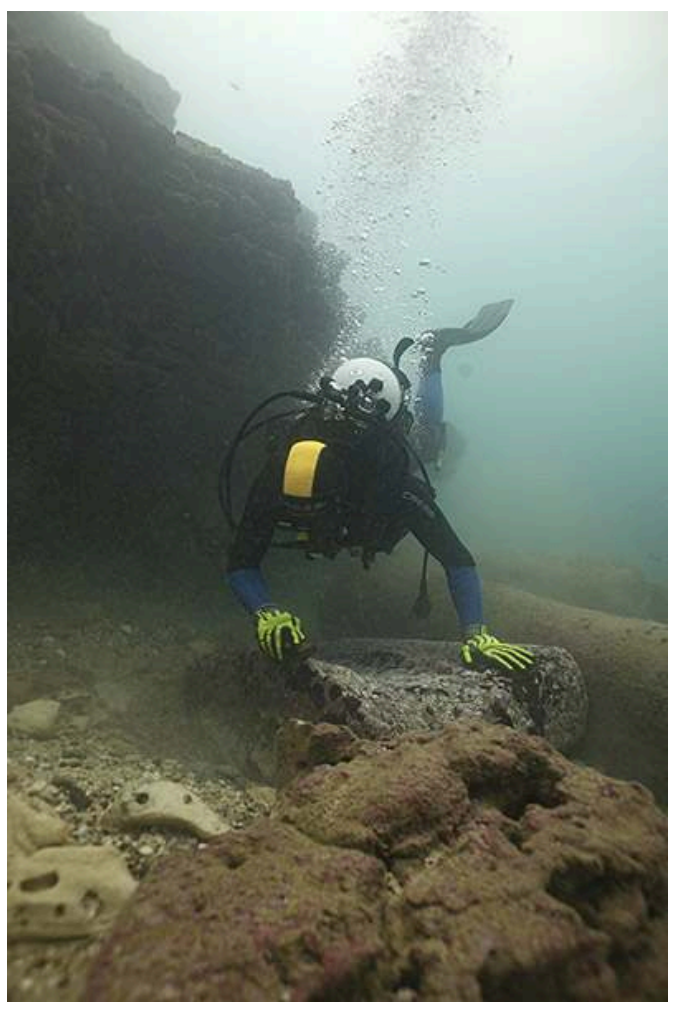

(c) Archives CEAlex. CEAlex_QB_2020_NDMPF_001

Fig. 114. Site du Phare. Déplacement d'un bloc pour l'acquisition photographique des faces cachées (P. Soubias).

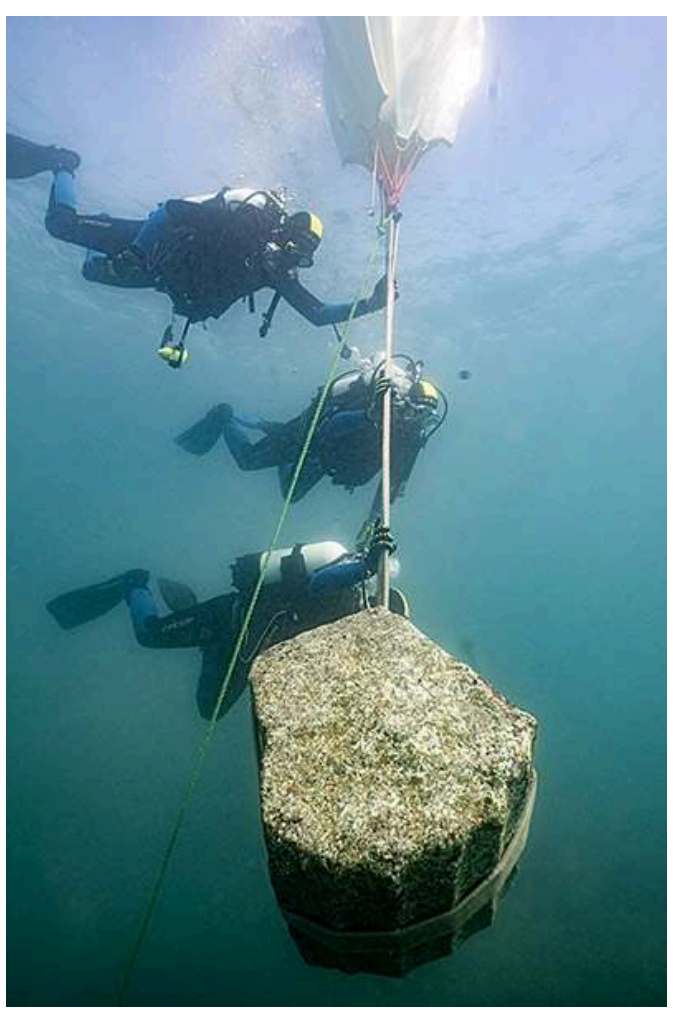

(C) Archives CEAlex. CEAlex_QB_2020_NDMPF_002 
Nous présentons ici un rapide descriptif des blocs :

- CEAlex 1295 (copie partielle) (fig. 115-116)

Profondeur $226 \mathrm{~cm} \times$ longueur cons. $200 \mathrm{~cm} \times$ H. c. $150 \mathrm{~cm}$.

Fragment de l'extrémité d'un linteau en granit rose de porte monumentale de style hellénique se raccordant au fragment CEAlex 1003.

Fig. 115. Site du Phare. Fragment CEAlex 1295 (P. Soubias).

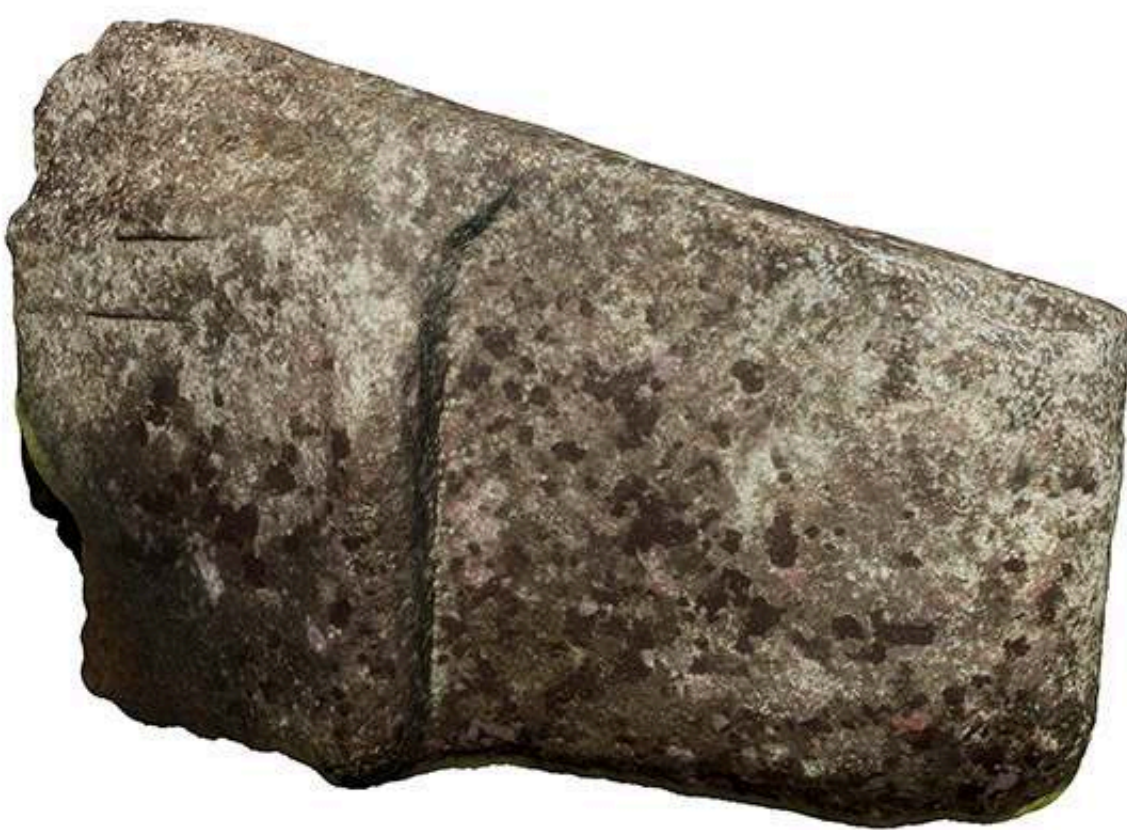

(C) Archives CEAlex. CEAlex_QB_2020_NDMPM_001 
Fig. 116. Site du Phare. Fragment CEAlex 1295 (P. Soubias).

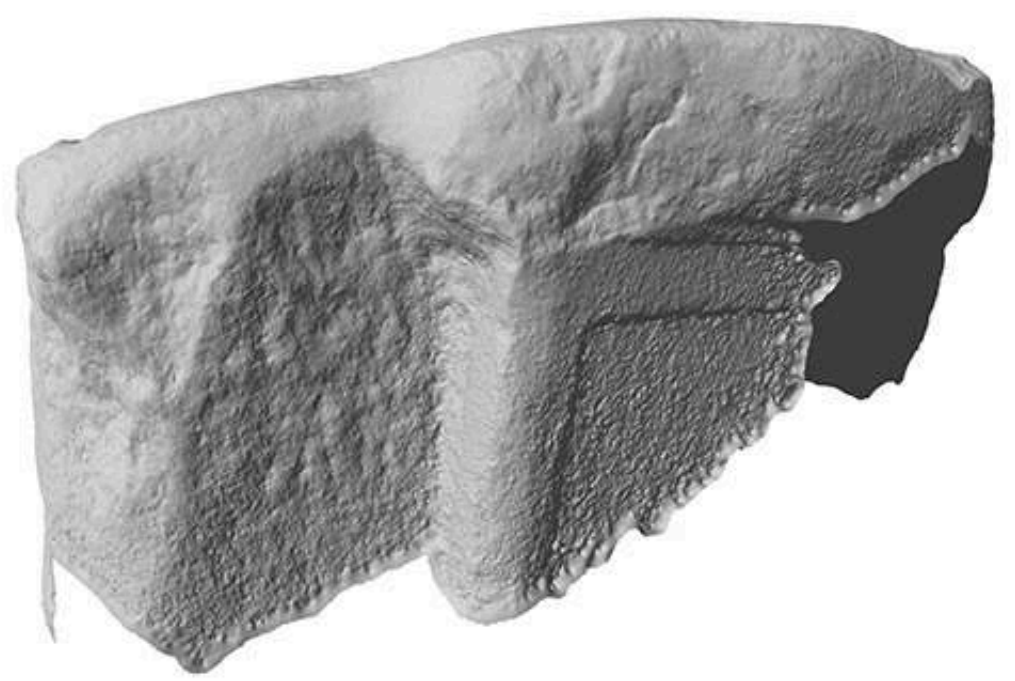

(C) Archives CEAlex. CEAlex_QB_2020_NDMPM_002

- CEAlex 2109 (copie partielle) (fig. 117-118)

H. cons ? $181 \mathrm{~cm}$; diam. sup. $84 \mathrm{~cm}$; diam. inf. $93 \mathrm{~cm}$.

Fragment de la partie haute d'un fût monolithique à cannelures en granit rose avec une mortaise de goujon cylindrique au centre du lit d'attente, et un bandeau d'anathyrose circulaire avec le centre démaigri et creusé à la pointe ; le fragment a été réemployé comme bloc courant, posé à plat et pour cela le fût a été aplani grossièrement (stries parallèles à la pointe) sur deux surfaces opposées. 
Fig. 117. Site du Phare. Fragment CEAlex 2109 (P. Soubias).

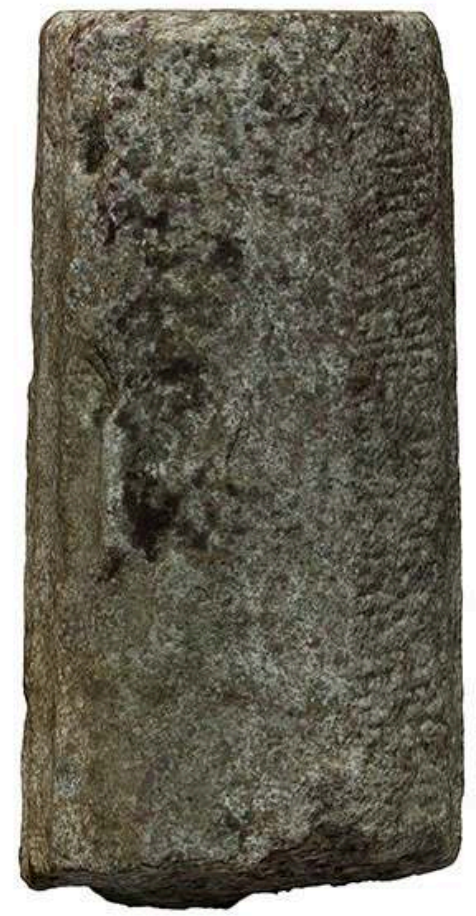

(c) Archives CEAlex. CEAlex_QB_2020_NDMPM_003

Fig. 118. Site du Phare. Fragment CEAlex 2109 (P. Soubias).

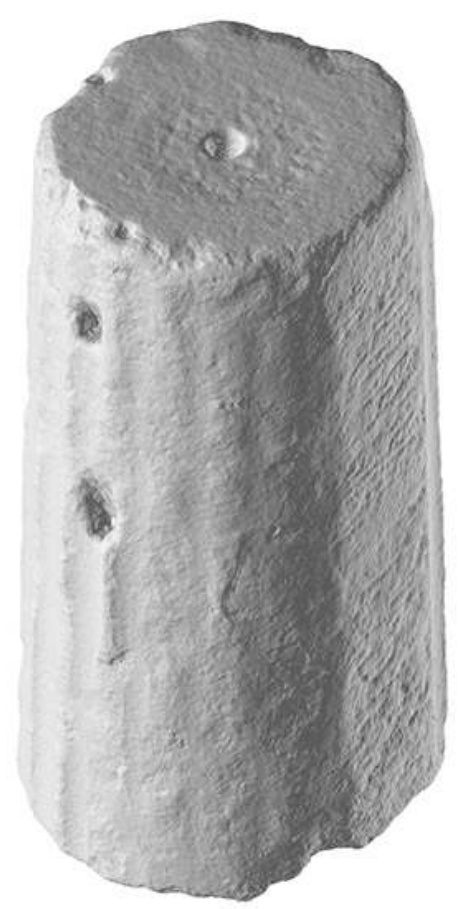

(c) Archives CEAlex. CEAlex_QB_2020_NDMPM_004 
- CEAlex 2174 (copie complète) (fig. 119-120)

Longueur cons. $112 \mathrm{~cm} \times \mathrm{H} .66 \mathrm{~cm} \times$ ép. $35 \mathrm{~cm}$.

Fragment d'angle d'une architrave en granite rose avec corniche à gorge égyptienne décorée de cartouches et d'uraei.

Fig. 119. Site du Phare. Fragment CEAlex 2174 (P. Soubias).

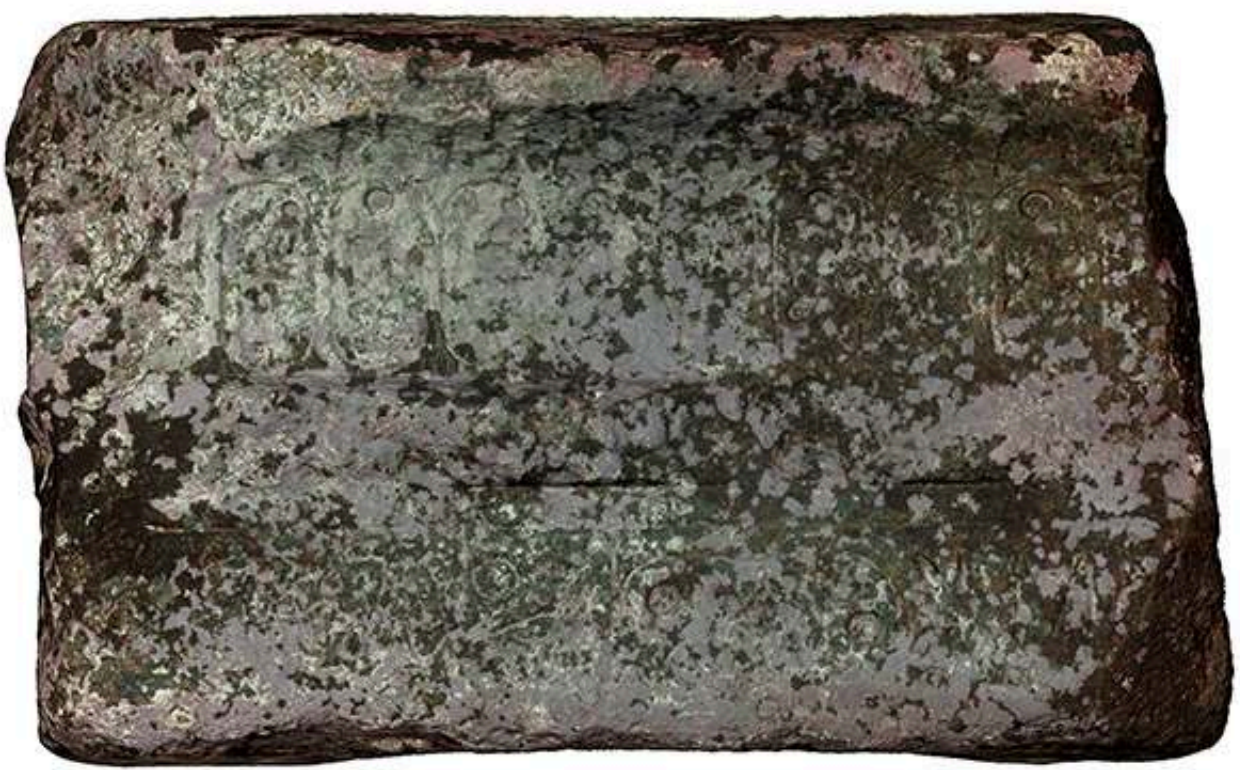

(c) Archives CEAlex. CEAlex_QB_2020_NDMPM_005 
Fig. 120. Site du Phare. Fragment CEAlex 2174 (P. Soubias).

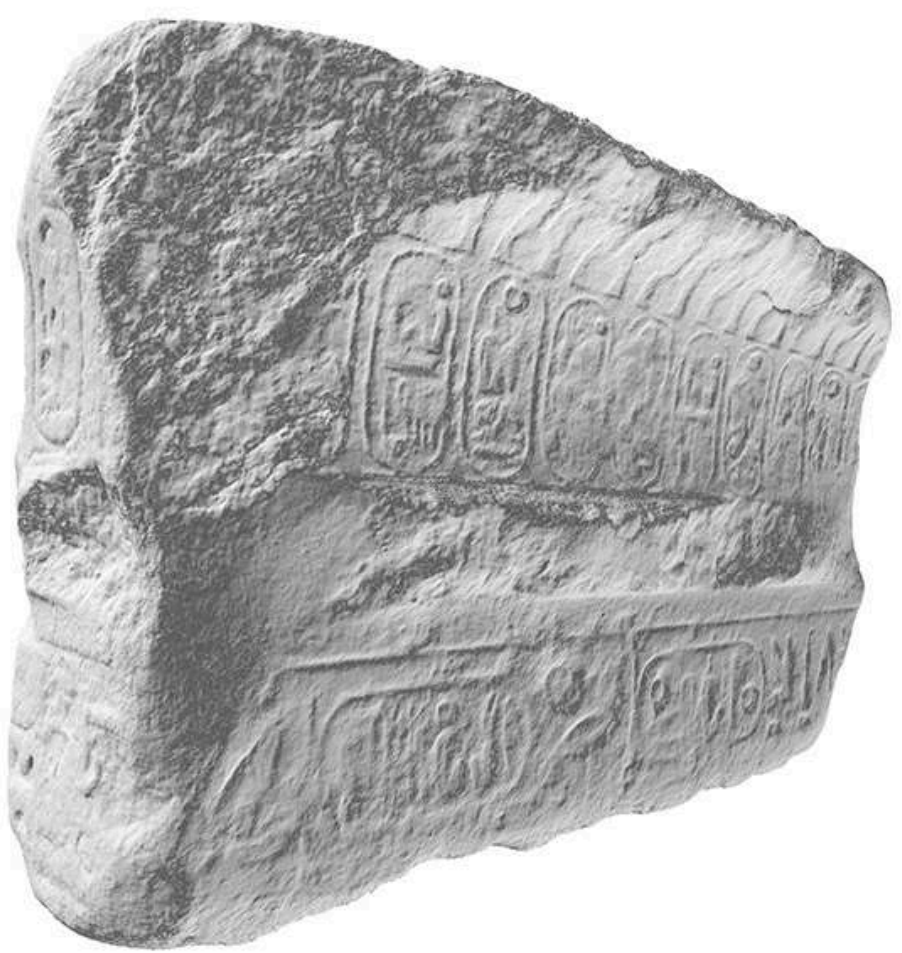

(c) Archives CEAlex. CEAlex_QB_2020_NDMPM_006

- CEAlex 2308 (copie partielle) (fig. 121-122)

Longueur cons. $177 \mathrm{~cm}$; largeur cons. $132 \mathrm{~cm} ; \mathrm{H} . \mathrm{c} .70 \mathrm{~cm}$.

Fragment de plinthe double avec trou de pince et cavité de scellement en Pi sur le lit d'attente; après abandon, le bloc a été débité par la technique des coins forcés; complément après désensablement de la copie réalisée en 2019. 
Fig. 121. Site du Phare. Fragment CEAlex 2308 (P. Soubias).

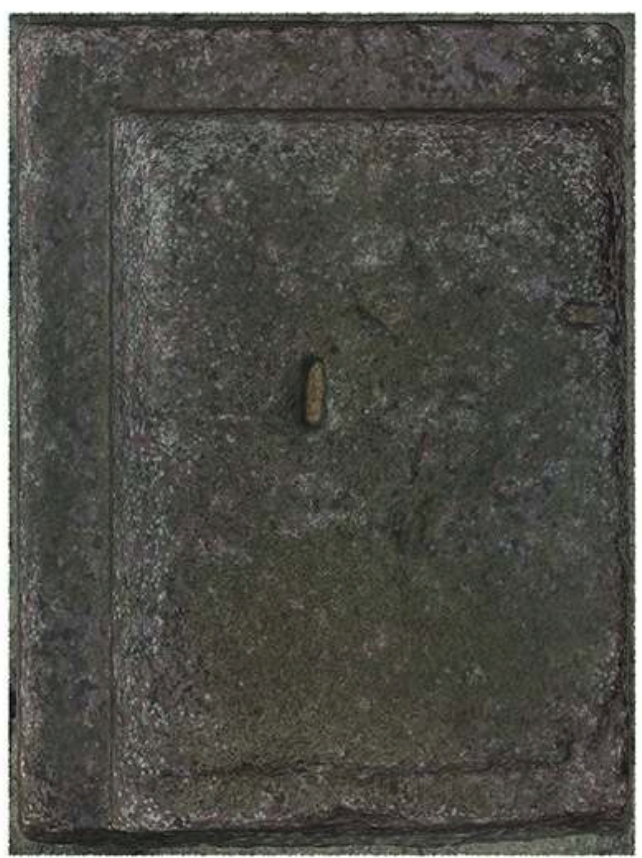

@ Archives CEAlex. CEAlex_QB_2020_NDMPM_007

Fig. 122. Site du Phare. Fragment CEAlex 2308 (P. Soubias).

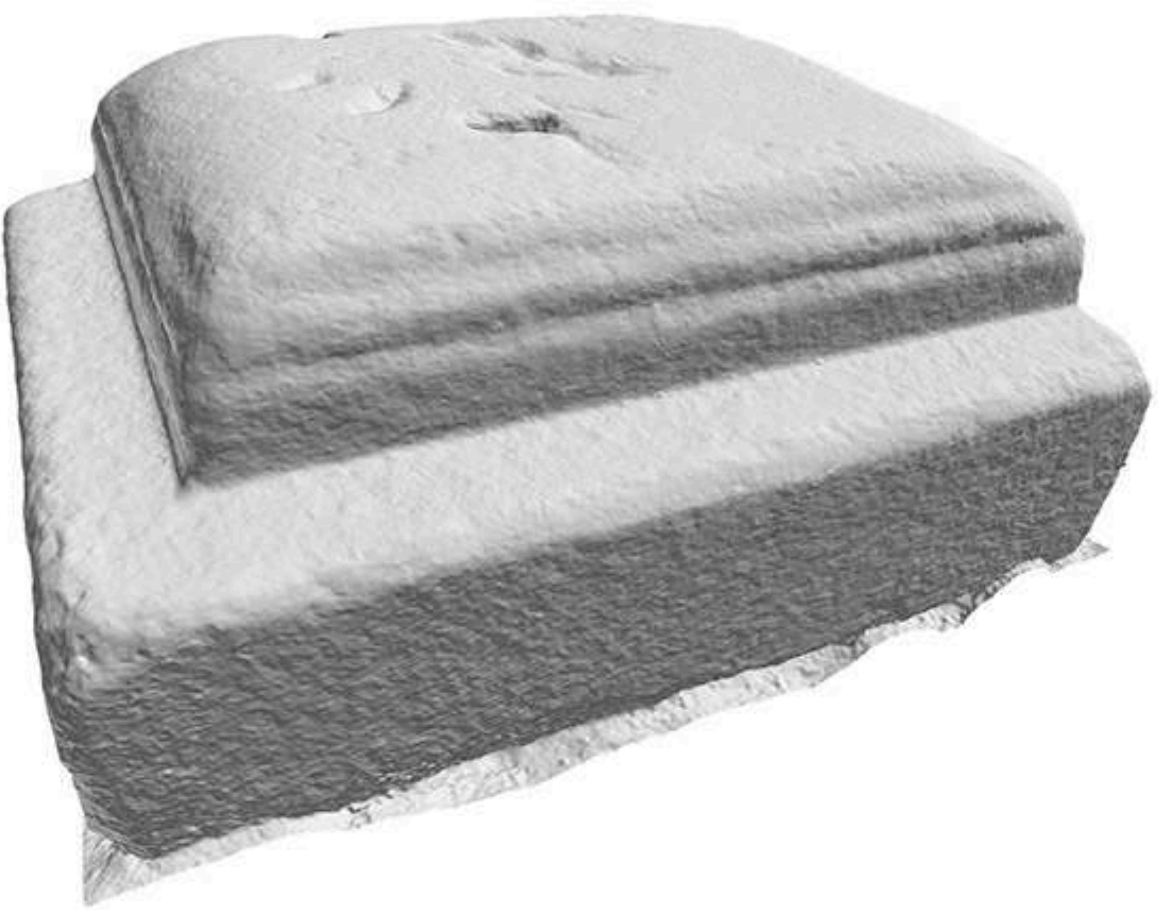

(C) Archives CEAlex. CEAlex_QB_2020_NDMPM_008 
- CEAlex 2407 (copie partielle) (fig. 123)

H. cons. $490 \mathrm{~cm}$; diam. sup. $56 / 57 \mathrm{~cm}$; diam. inf. $85 / 86 \mathrm{~cm}$.

Fragment de colonne monolithique à fût lisse en granit rose avec base conservée faite d'un listel plat et d'un congé.

Fig. 123. Site du Phare. Fragment CEAlex 2407 (P. Soubias).

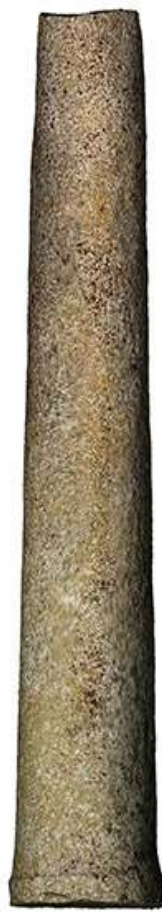

(C) Archives CEAlex. CEAlex_QB_2020_NDMPM_009

- CEAlex 2412 (copie complète) (fig. 124-125)

H. cons. $104 \mathrm{~cm}$; diam. sup. c. $114 \mathrm{~cm}$; diam. inf. c. 128/130 cm.

Fragment d'un fût ou d'un tambour à cannelures en granite rose portant sur son lit de pose ou d'attente un bandeau d'anathyrose circulaire avec un centre démaigri à la pointe; le bloc a été retaillé pour un autre usage ou comme pièce rapportée pour restaurer une colonne qui aurait été endommagée. 
Fig. 124. Site du Phare. Fragment CEAlex 2412 (P. Soubias).

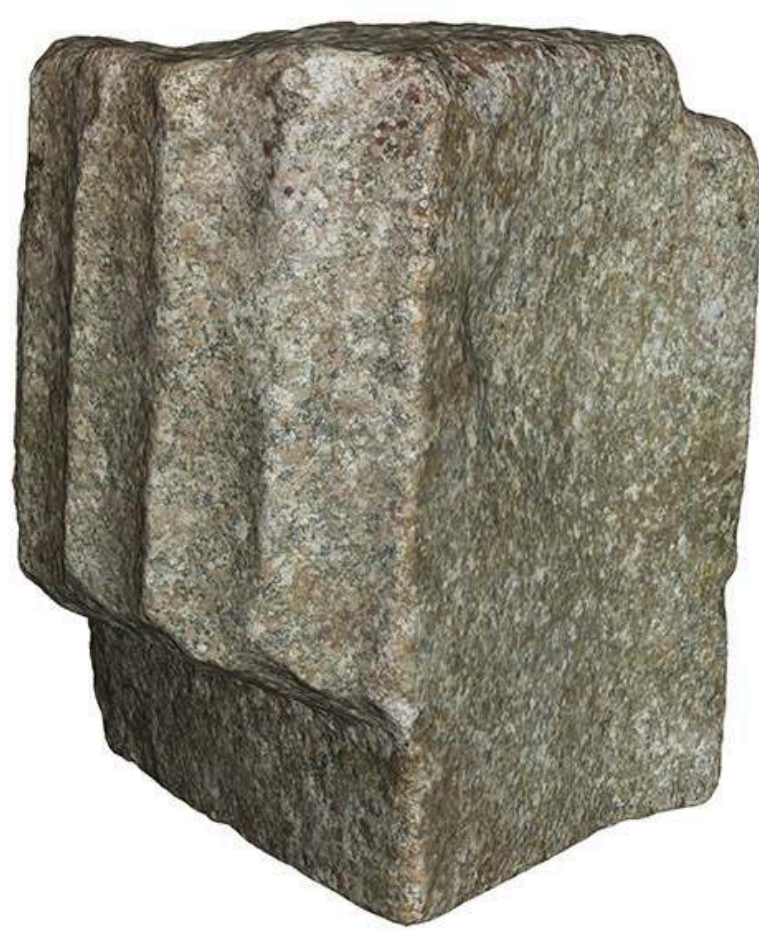

(c) Archives CEAlex. CEAlex_QB_2020_NDMPM_010

Fig. 125. Site du Phare. Fragment CEAlex 2412 (P. Soubias).

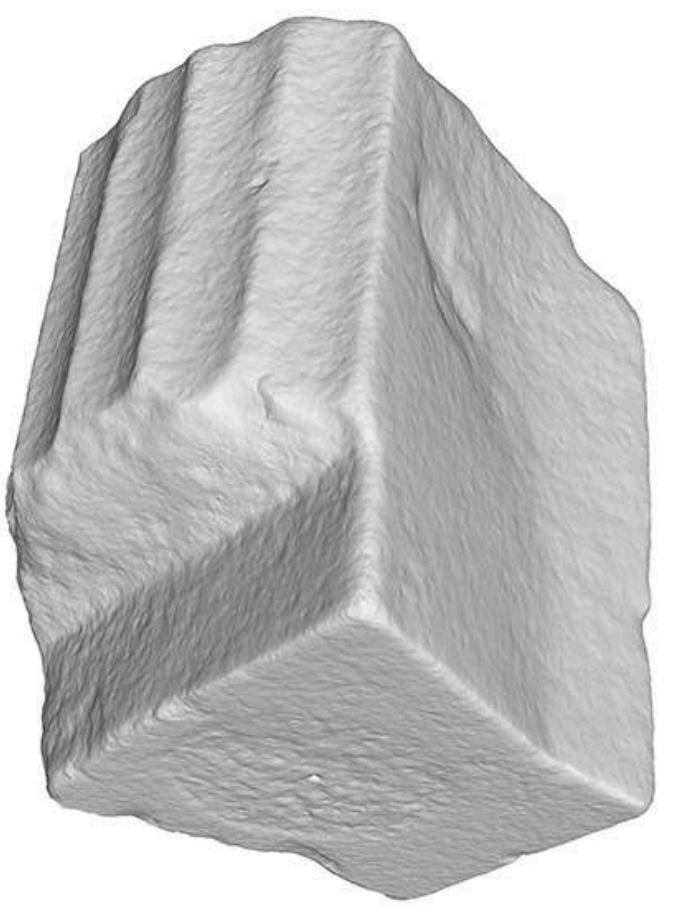

(c) Archives CEAlex. CEAlex_QB_2020_NDMPM_011 
- CEAlex 2485 (copie complète) (fig. 126-127)

H. cons. $87 \mathrm{~cm}$; diam. sup. c. $90 \mathrm{~cm}$; diam. inf. c. $86 \mathrm{~cm}$.

Fragment d'un fût ou d'un tambour à cannelures en granite rose portant sur son lit de pose ou d'attente un bandeau d'anathyrose circulaire avec un centre fortement démaigri et creusé à la pointe ; dans l'espace du bandeau, deux cavités de scellement en Pi sont creusées, indiquant que ce bloc a fait l'objet d'une réparation (le fragment a été scellé sans doute dans le cadre du remontage du monument auquel il appartenait). Enfin, il porte deux cavités d'emboîture de coin d'équarrissage indiquant qu'un débitage du fût a été réalisé après son abandon.

Fig. 126. Site du Phare. Fragment CEAlex 2485 (P. Soubias).

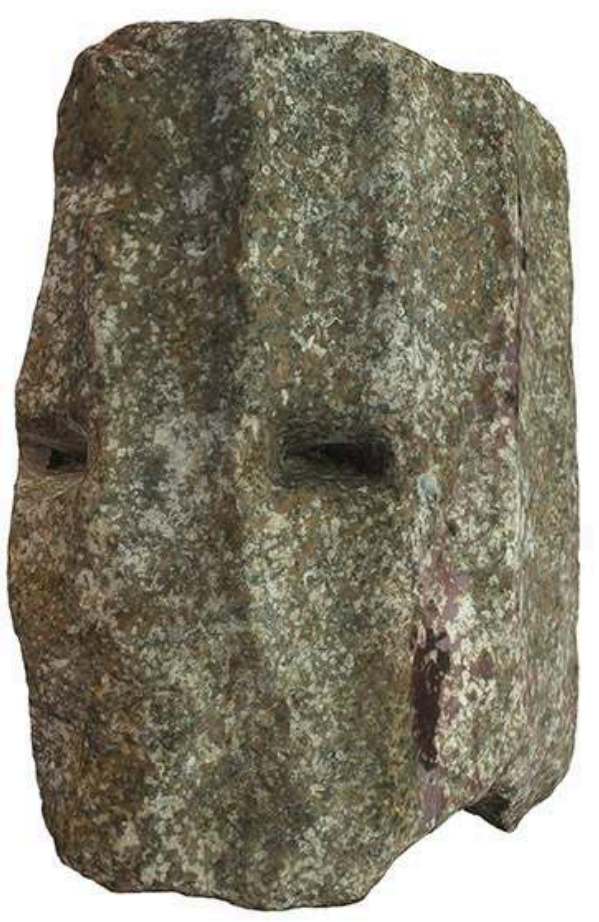


Fig. 127. Site du Phare. Fragment CEAlex 2485 (P. Soubias).

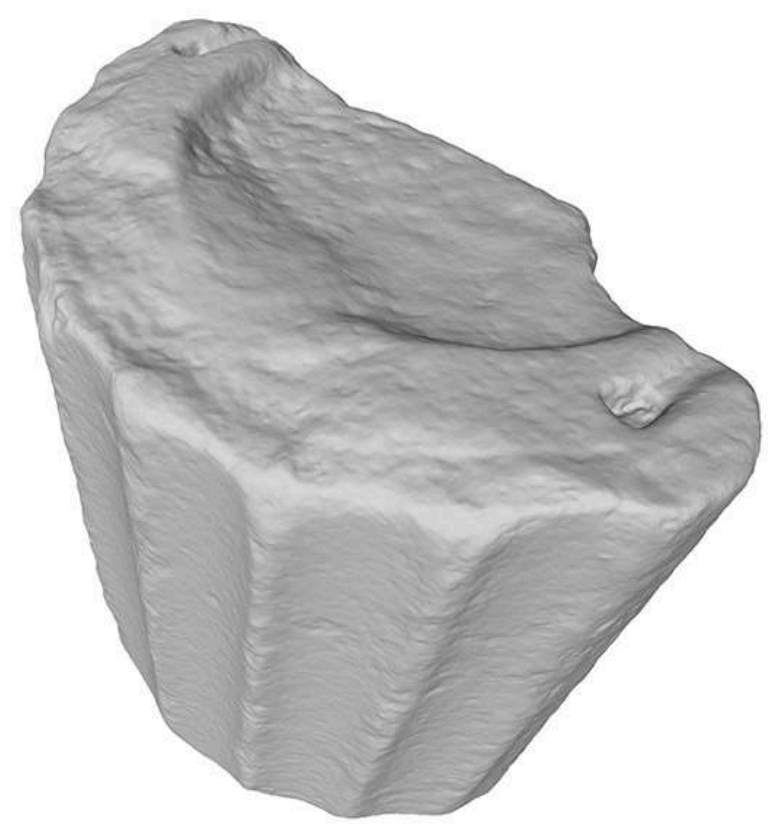

(c) Archives CEAlex. CEAlex_QB_2020_NDMPM_013

- CEAlex 2902 (copie complète) (fig. 128-129)

Longueur cons. $106 \mathrm{~cm}$; largeur cons. $80 \mathrm{~cm} ; \mathrm{H} .66 / 67 \mathrm{~cm}$

Fragment de plinthe double débitée après abandon (technique des coins forcés).

Fig. 128. Site du Phare. Fragment CEAlex 2902 (P. Soubias).

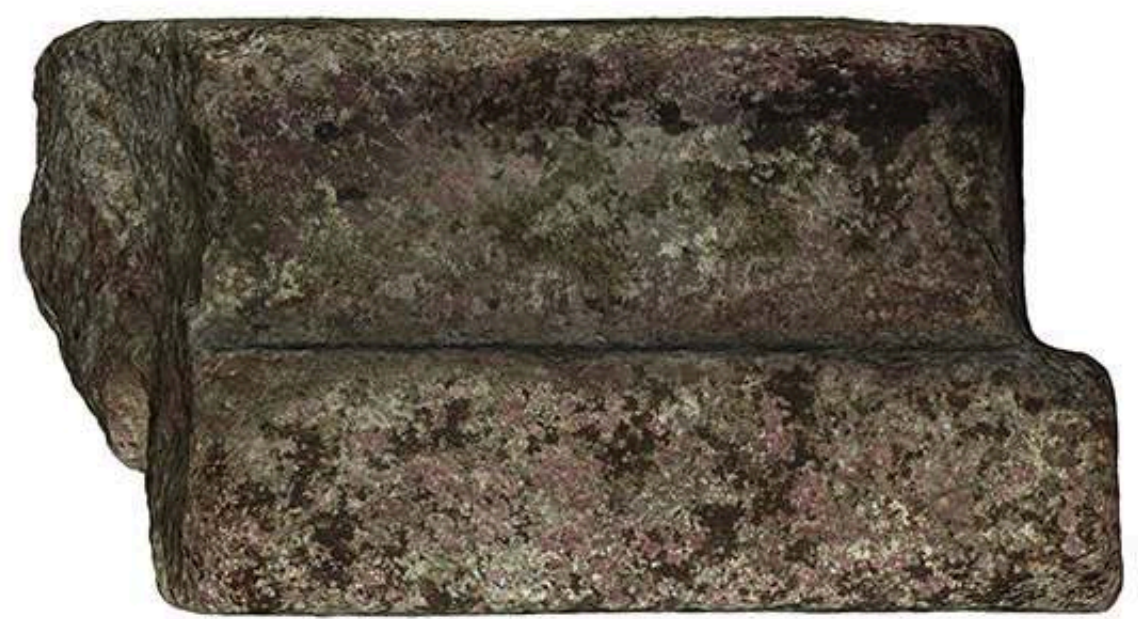

(c) Archives CEAlex. CEAlex_QB_2020_NDMPM_014 
Fig. 129. Site du Phare. Fragment CEAlex 2902 (P. Soubias).

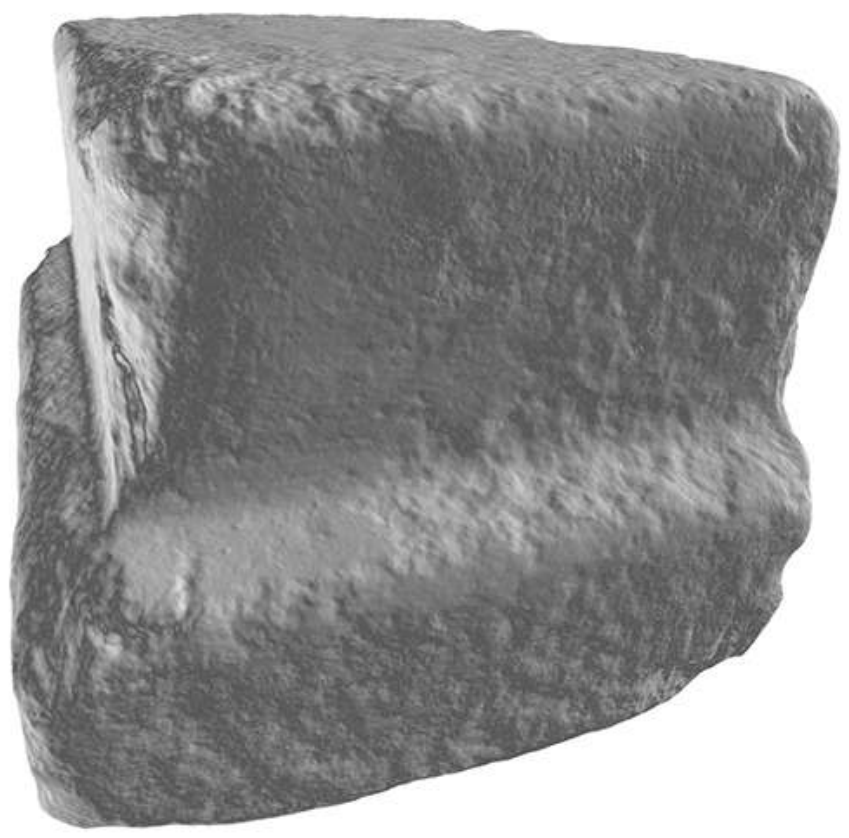

(c) Archives CEAlex. CEAlex_QB_2020_NDMPM_015

130 Une étude plus aboutie des 3 fragments de fût cannelé (CEAlex 2109, 2412 et 2485) permettra d'envisager l'éventualité qu'ils fassent partie de la même colonne. Ces nouvelles copies numériques portent à 161 le nombre de blocs modélisés sur le site.

\subsection{Compléments cartographiques}

"L'archéologie est une affaire de cartographie ». Rapportée à un site immergé de cette taille, cette maxime prend tout son sens. L'orthophotoplan «vrai» et le modèle numérique de surface du site enregistrent les coordonnées de chaque point, donc la position précise de chaque objet ou bloc. Le travail d'acquisition réalisé en 2014, 2015, 2016, 2018, 2019 et 2020 nous a permis de créer, puis de mettre à jour une carte topographique à haute résolution et de documenter de la façon la plus complète possible, au travers d'un SIG, le site sous-marin (fig. 130). 
Fig. 130. Site du Phare. Carte générale du site sous-marin montrant les données réunies issues d'une part de l'ancienne carte du site (1994-2014) et d'autre part du traitement des orthophotoplans (2014-2020) (I. Hairy).

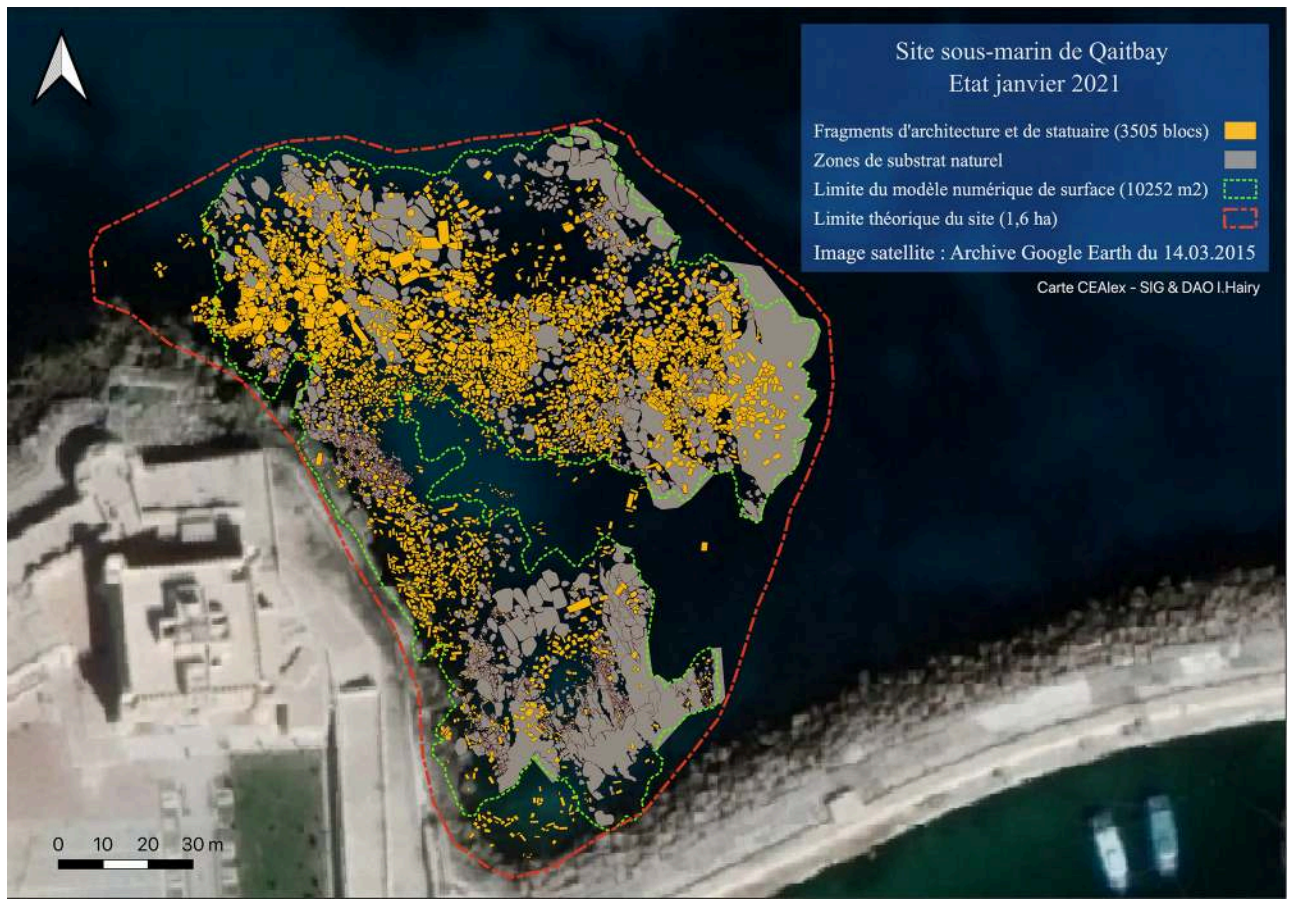

(c) Archives CEAlex. CEAlex_QB_2020_NDMCN_011

L'étude des différents orthophotoplans intégrés au SIG vient compléter année après année la base de données issue de l'ancienne carte du site produite et gérée sous Autocad en 1994 puis sous MapInfo à partir de 1996. Le traitement des données issues des orthophotoplans est encore en cours; ce travail permet d'afficher au 31 janvier 2020 un nombre de 3505 blocs d'architecture ou de statuaire présents sur le site et de présenter un dessin plus abouti du relief naturel en distinguant le substrat des zones ensablées. Sur les zones de recouvrement, les orthophotoplans révèlent les transformations naturelles du site d'une année à l'autre, notamment les variations de l'ensablement des dénivelés (fig. 131-132).

Fig. 131. Site du Phare. Variations d'ensablement dans la zone 1 du site (I. Hairy).

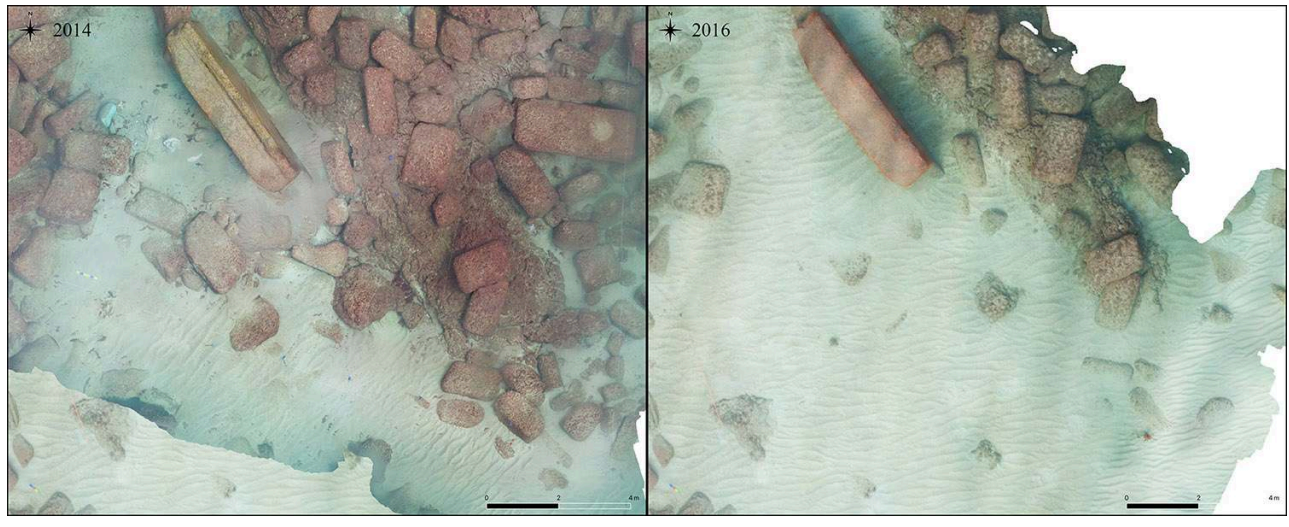

(c) Archives CEAlex. CEAlex_QB_2020_NDMPF_003 
Fig. 132. Site du Phare. Variations d'ensablement dans la zone 3 du site (I. Hairy).

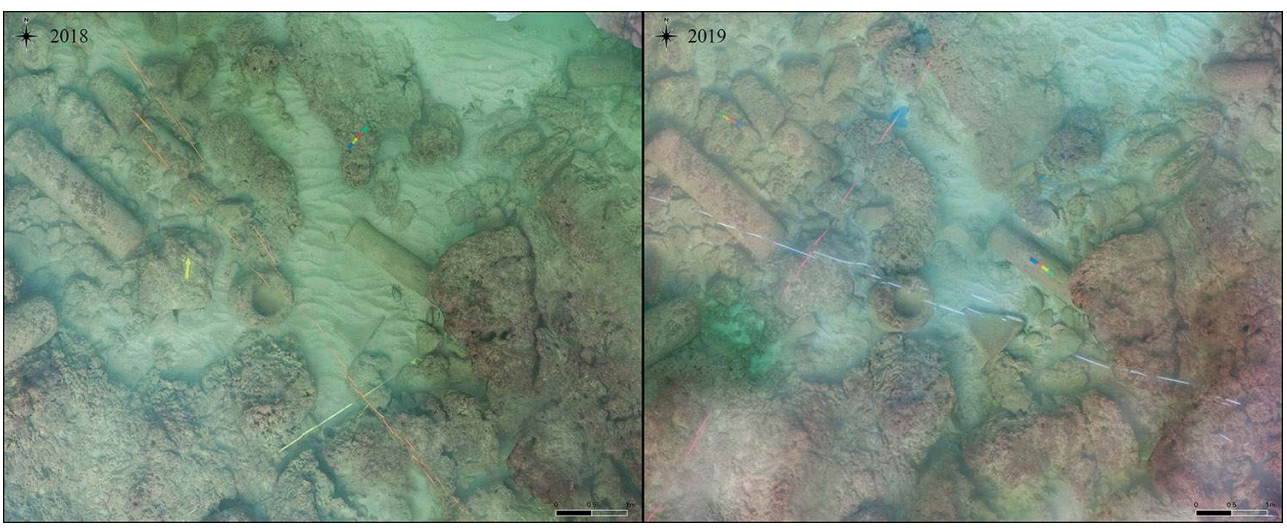

(c) Archives CEAlex. CEAlex_QB_2020_NDMPF_004

\section{BIBLIOGRAPHIE}

\section{ABD EL-FATTAH 1998}

Ahmed Abd el-Fattah, « Recent Discoveries in Alexandria and the Chora », in Jean-Yves Empereur (éd.), Commerce et artisanat dans l'Alexandrie hellénistique et romaine. Actes du Colloque d'Athènes organisé par le CNRS, le laboratoire de céramologie de Lyon et l'école française d'Athènes : 11-12 décembre 1988, BCH-Suppl. 33, 1998, Athènes, EfA, p. 37-53.

AWAD sous presse

Ismaël Awad, « An Archaeological Map of the Mareotid. The Principles, Methods and Potential of a GIS ", in Bérangère Redon (éd.), Western Borders and Margins of Egypt from Antiquity to the Middle Age, actes de colloque, Le Caire, 2-3 decembre 2017, Le Caire, Ifao, sous presse.

BABRAJ, KOGUT, ZIEBA 2012

Krzysztof Babraj, Janusz P. Khogut, Jakub ZiĘba, « Numerical Reconstruction of Behaviour of the Ancient Pottery Kiln from Marea (Egypt) Due to Operational Loading », in Jerzy Jasieńko (éd.), Structural Analysis of Historical Construction: Proceedings of the [8th] International Conference on Structural Analysis of Historical Constructions, SAHC 2012, 15-17 October, Wroclaw, Poland, Wrocław, DWE, 2012, p. 1003-1014.

BLUE, KHALIL 2011 (LRMP)

Lucy K. Blue, Emad Khalil, A Multidisciplinary Approach to Alexandria's Economic Past: The Lake Mareotis Research Project, BAR-IS 2285, Oxford, British Archaeological Reports, 2011.

BUTZER 1960

Karl W. Butzer, « On the Pleistocene Shore Lines of Arabs' Gulf, Egypt », The Journal of Geology 68/6, 1960, p. 626-637.

EL-ASHMAWY 1998

Feisal El-Ashmawi, « Pottery Kiln and Wine-Factories at Burg El-Arab », in Jean-Yves Empereur (éd.), Commerce et artisanat dans l'Alexandrie hellénistique et romaine. Actes du Colloque 
d'Athènes organisé par le CNRS, le laboratoire de céramologie de Lyon et l'école française d'Athènes :

11-12 décembre 1988, BCH-Suppl. 33, 1998, Athènes, EfA, p. 55-64.

EMPEREUR, PICON 1998

Jean-Yves Empereur, Maurice Picon, «Les ateliers d'amphores du lac Mariout », in Jean-Yves Empereur (éd.), Commerce et artisanat dans l'Alexandrie hellénistique et romaine. Actes du Colloque d'Athènes organisé par le CNRS, le laboratoire de céramologie de Lyon et l'école française d'Athènes : 11-12 décembre 1988, BCH-Suppl. 33, 1998, Athènes, EfA, p. 75-91.

GROSSMANN, KHORSID 1994

Peter Grossmann, Fathy Khorsid, « The Biapsidal Church at Sidi Mahmud (Burg el-Arab) in Maryut », BSAC 33, 1994, p. 79-90.

PICHOT sous presse

Valérie Pichot, « The Early Imperial Era Wine Amphora Workshop of Akademia (Mareotid) », Delta Survey Workshop Journal de Recherches, université de Mansoura, Mansoura, sous presse.

PICHOT, FLAUX 2015

Valérie Pichot, Clément Flaux, « Les fours à amphores du Haut-Empire du site d'Akadémia (Maréotide) : campagne de fouille et carottages 2014 », BCE 25, 2015, p. 259-276.

PICHOT, ŞENOL 2014

Valérie Pichot, Kaan şenol, « The Site of Akademia: The Amphora Workshop of Apol(l)ônios. First Excavation Campaign (July-August 2012) », BCE 24, 2014, p. 225-240.

PICHOT, ŞENOL 2015

Valérie Pichot, Kaan şenol, « Amphores de Gaza et résine sur le site de production d'amphores du Haut- Empire d'Akadémia (Maréotide) », BCE 25, 2015, p. 277-289.

PICHOT, ŞENOL Sous presse

Valérie Pichot, Kaan şenol, « The Site of Akademia. First Excavation Campaign (July-August 2012) on the Amphora Workshop of Apol(l)ônios ", Per Terram, Per Mare: Production and Transport of Roman Amphorae in the Eeastern Mediterranean, actes du colloque international de Nicosie, 12-15 avril 2013, université de Chypre, Nicosie, sous presse.

PICHOT, SIMONY sous presse

Valérie Pichot, avec la collaboration d'Aude Simony, « An Archaeological Map of the Mareotid: Initial Results Regarding the Evolution of Occupation in the Region », in Bérangère Redon (éd.), Western Borders and Margins of Egypt from Antiquity to the Middle Age, actes de colloque, Le Caire, 2-3 decembre 2017, Le Caire, Ifao, sous presse.

\section{NOTES}

1. Mieczyslaw D. Rodziewicz, Hellenistic Painted Goblets from Alexandria, EtudAlex 49, Alexandrie, Centre d'études alexandrines, 2020; Marie-Dominique Nenna (éd.) Alexandrina 5, EtudAlex 50, Alexandrie, Centre d'études alexandrines, 2020 ; Ingrid LAUBE, Excavations in the Basileia of Alexandria: Findings of the 1901 Ernst von Sieglin expedition, EtudAlex 51, Alexandrie, Centre d'études alexandrines, 2020; Delphine Dixneuf, La céramique issue des fouilles menées autour de la citerne el-Nabih (Alexandrie). De l'antiquité tardive à l'époque ottomane, EtudAlex 52, Alexandrie, Centre d'études alexandrines, 2020. 
2. Marie-Delphine Martellière, La Presse francophone d'Alexandrie, cat. expo., Institut français d'Égypte, Alexandrie, 10 novembre 2019 - 12 janvier 2020, Alexandrie, CEAlex, 2020 ; Marie-Dominique Nenna, Valérie Pichot, avec la collab. d'Ismaël Awad, Nicolas morand et Aude simony, Découvrir la campagne alexandrine, Alexandrie, 2020.

3. Pour les campagnes antérieures, voir les rapports d'activité du CEAlex, en ligne sur les sites web de l'Ifao et du CEAlex https://www.cealex.org/le-cealex/rapportsactivites/; et pour les activités en 2019, https://journals.openedition.org/baefe/1094.

4. Cet ensemble de structures dégagé lors de la campagne 2019 se compose du four 20044, du foyer 20045, des niveaux cendreux 20040 et 20041, de la structure de briques 20036 et des blocs calcaires 20061.

5. EMPEREUR, PICON 1998, p. 87, atelier $n^{\circ} 22$.

6. Si les différentes phases d'activité sont en cours d'identification, il restera néanmoins difficile de proposer une durée d'occupation de l'atelier étant donné la pauvreté du site en mobilier permettant d'affiner la chronologie, comme la céramique importée, les monnaies...

7. PICHOT, ŞENOL 2014 ; PICHOT, ŞENOL sous presse.

8. PICHOT, FLAUX 2015 ; PICHOT, ŞENOL 2015.

9. PICHOT sous presse.

10. Étude, analyses et interprétations : V. Pichot (CEAlex, CNRS, USR 3134).

11. Étude, analyses et interprétations : C. Flaux (Mosaïques Archéologie).

12. Étude du mobilier amphorique: K. şenol (université de l'Égée, Izmir); étude du mobilier céramique: Delphine Dixneuf (LA3M, CNRS, UMR 7298); étude des outils : V. Pichot (CEAlex, CNRS, USR 3134); étude des monnaies: Thomas Faucher (IRAMAT, CNRS, UMR 5060) et Olivier Lempereur (HiSoMA, CNRS, UMR 5189) ; étude de la faune : N. Morand (Fondation Fyssen, Institut royal des Sciences naturelles de Belgique, Bruxelles) ; étude botanique effectuée en 2014 : Thierry Otto (Ecolab, CNRS, UMR 5245).

13. Analyses et interprétations: Mai Abdel Gawad (Service de caractérisation des matériaux du CEAlex).

14. Analyses et interprétations: Assem Bahnasy (Service de caractérisation des matériaux du CEAlex).

15. Pour les campagnes antérieures, voir les rapports d'activité du CEAlex, en ligne sur les sites web de l'Ifao et du CEAlex https://www.cealex.org/le-cealex/rapportsactivites/; et pour les activités en 2019, https://journals.openedition.org/baefe/1094.

16. Le four oriental (secteur 7) a un diamètre intérieur de 7,68 $\mathrm{m}$ et un diamètre extérieur de 12,65 m, avec une épaisseur des murs de $2 \mathrm{~m}$; le four de Borg el-Arab (ELASHMAWY 1998) a un diamètre intérieur de 7,60 $\mathrm{m}$ et un diamètre extérieur de 12,60 m; le four de «Maréa »-Philoxénité (BABRAJ, KOGUT, ZIEBA 2012) possède un diamètre intérieur estimé à environ $8 \mathrm{~m}$; celui de Margham de $7 \mathrm{~m}$, pour un diamètre intérieur de 6 m (ABD EL-FATTAH 1998, fig. 8-13).

17. Altitude supérieure de la sole $4,95 \mathrm{~m}$, épaisseur estimée à environ $0,50 \mathrm{~m}$; altitude supérieure du mur circulaire conservé $5,66 \mathrm{~m}$ à l'ouest et 5,03 $\mathrm{m}$ au sud-est.

18. Les prospections que mènent le CEAlex en Maréotide ont permis d'augmenter le nombre d'ateliers connus. Pour un état des lieux sur les ateliers et plus largement sur l'occupation de la Maréotide et son évolution, voir Valérie Pichot, Aux portes 
d'Alexandrie. Le développement de la Maréotide hellénistique et romaine, thèse de doctorat obtenu en 2017, publication en préparation dans les collections du CEAlex. L'ensemble des ateliers encore existants et accessibles ont fait l'objet de prélèvement d'échantillons lors des prospections: une étude interdisciplinaire (archéologie, céramologie, chimie et pétrographie) est menée actuellement par le CEAlex (M. Abdelgawad, A. Bahnasy, V. Pichot, A. Simony) pour la caractérisation de chaque atelier ainsi que pour la caractérisation des productions de la région de Maréotide. Cette étude sur les ateliers hellénistiques et romains de la région sera publiée prochainement.

19. Ce phénomène avait déjà été noté lors des fouilles de la presqu'île de "Maréa " (2003-2012, fouilles du CEAlex sous la direction de V. Pichot) et est confirmée par la mise en parallèle de données archéologiques et géomorphologiques issues du four oriental et du puits de la sakieh occidentale d'Akadémia, РICHOT, FLAUX 2015.

20. Pour des premières présentations, voir AWAD sous presse et PICHOT, SIMONY sous presse. Pour les campagnes antérieures, voir les rapports d'activité du CEAlex, en ligne sur les sites web de l'Ifao et du CEAlex https://www.cealex.org/le-cealex/rapportsactivites/; et également pour les activités en 2019, https://journals.openedition.org/ baefe/1094.

21. Nous rappelons ici que le paysage de la Maréotide est constitué d'une succession de dunes fossiles (nous conservons ici le terme ride, qui est largement employé, même si, dans la terminologie géologique, il s'agit de crêtes) et de dépressions qui ont joué un grand rôle dans la mise en place de l'occupation de la région. Les rides sont numérotées de I à VIII du nord au sud (voir BUTZER 1960, p. 626-637). Alexandrie et Taposiris Magna sont installées sur la ride II et le bras occidental du Mariout se développe entre la ride II et la ride III.

22. BLUE, KHALIL 2011, p. 130-135.

23. GROSSMANN, KHORSID 1994, p. 79-90.

24. Le nom correct de ce site est Sidi 'Abdallah el-Shayet, et non Karm Abou Hamdah localisé plus au sud sur les cartes et qui a aujourd'hui totalement disparu.

25. Le boîtier utilisé jusque-là était un Nikon D700 avec un objectif $24 \mathrm{~mm}$; l'appareil a dû être remplacé cette année par un Panasonic DMC-GH4 Crop-frame.

26. Ground Control Point.

27. Ce système de repérage a été mis en place en 2019 pour pallier les problèmes de visibilité récurrents générés par la mise en place de la digue de protection du fort en pleine mer, au nord-ouest du site. Les points sont espacés en moyenne de 11 à $15 \mathrm{~m}$.

28. Le SIG du site de Qaitbay était jusque-là géré sous MapInfo. Les fichiers anciens sont en cours de conversion pour être intégrés dans QGIS, logiciel sous lequel a été créée la nouvelle carte du site.

29. Le site était estimé à environ 1,3 hectares avant 2019. 


\section{INDEX}

Thèmes : CEAlex

lieux https://ark.frantiq.fr/ark:/26678/pcrtOTIv0VQNwW, https://ark.frantiq.fr/ark:/26678/ pcrtsgWZ4lzKyf

sujets https://ark.frantiq.fr/ark:/26678/pcrtYhYMiLwDUr, https://ark.frantiq.fr/ark:/26678/ pcrtuWZ3xzYnjx, https://ark.frantiq.fr/ark:/26678/pcrtf1euItAGSn, https://ark.frantiq.fr/ark:/ 26678/pcrtb0B7tP2XnB, https://ark.frantiq.fr/ark:/26678/pcrtb1E0Dz7cSX, https:// ark.frantiq.fr/ark:/26678/crtjlFn2fZdjL, https://ark.frantiq.fr/ark:/26678/pcrtM6WKp5XFlj, https://ark.frantiq.fr/ark:/26678/pcrtHBU3mnn3DF, https://ark.frantiq.fr/ark:/26678/ pcrtHIjtOg1P75

Année de l'opération : 2020

nature https://ark.frantiq.fr/ark:/26678/pcrtb1E0Dz7cSX

chronologie https://ark.frantiq.fr/ark:/26678/crtHzOwp9KOps, https://ark.frantiq.fr/ark:/ 26678/pcrtHWdyk0IJbG

\section{AUTEURS}

\section{AUDE SIMONY}

Archéologue-céramologue, CNRS, CEAlex

\section{KATHRIN MACHINEK}

Archéologue-architecte, CNRS, CEAlex

\section{GEORGES SOUKIASSIAN}

Archéologue, CNRS, CEAlex

\section{VALÉRIE PICHOT}

Archéologue, CNRS, CEAlex

\section{ISMAËL AWAD}

Ingénieur topographe-cartographe, CEAlex

\section{MOHAMED ABDELAZIZ}

Traitement photogrammétrique

PHILIPPE SOUBIAS

Photographe, CNRS, CEAlex 